\title{
XVI meeting of the European Association for Haematopathology 2012 Abstracts
}

\section{Bone Marrow Symposium}

\section{$\underline{\text { BMS001 }}$}

\section{Application of a BRAF V600E mutation specific antibody for the diagnosis of Hairy Cell Leukemia}

Mindaugas Andrulis ${ }^{1}$; Roland Penzel ${ }^{2}$; Wilko Weichert ${ }^{2}$; Andreas von Deimling ${ }^{3}$; David Capper ${ }^{3}$

(1) Institute of Pathology Heidelberg (2) Department of General Pathology, Institute of Pathology, Ruprecht-KarlsUniversity Heidelberg, Heide (3) Department of Neuropathology, Institute of Pathology, Ruprecht-Karls-University Heidelberg, Heidelberg, Germany

Background: Recently BRAF V600E mutations have emerged as genetic hallmark of hairy cell leukemia (HCL). This specific point mutation is present in virtually all cases of HCL but exceedingly rare in other peripheral B-cell neoplasms.

Aim of the study: In this study we investigated the application of a BRAF V600E mutation specific antibody (clone VE1) to differentiate HCL from HCL mimics, such as HCLvariant and splenic marginal zone lymphoma.

Materials and methods: A total of 52 routinely processed formalin fixed paraffin embedded (FFPE) tissue specimens were investigated (bone marrow $n=46$; spleen $n=6$ ) for expression of V600E mutated BRAF protein.

Results: All 32 cases of HCL were scored positive, all non HCL cases were scored negative. In 28 of $30 \mathrm{HCL}$ cases the presence of a BRAF V600E mutation could be confirmed by direct sequencing while no BRAF mutations were detected among 20 HCL-mimics. We further screened 228 mature Bcell neoplasms with VE1 and detected one positive case of B-lymphocytic leukemia. Sequencing confirmed the presence of a BRAF V600E mutation.
Conclusions: In conclusion we demonstrate the high sensitivity and specificity of VE1 immunohistochemistry for HCL. This on-slide technique might be particularly helpful in interpreting challenging biopsies with low tumor content.

\section{BMS002}

Large granular T-lymphocyte expansion following allogeneic hematopoietic stem cell transplantation

Alexander Tzankov ${ }^{1}$; Sabine Nann²${ }^{2}$;örg Halter $^{2}$; Dominik $\mathrm{Heim}^{2}$; Dimitrios Tsakiris ${ }^{2}$; Caroline Arber ${ }^{2}$; Andreas Buser $^{2}$; Alois Gratwohl ${ }^{2}$; Andre Tichelli ${ }^{2}$; Alicia Rovo ${ }^{2}$

(1) Institute of Pathology, University Hospital Basel, Switzerland (2) Hematology, University Hospital Basel

Background: Reactive, usually transient, expansions of CD3 positive large granular T-lymphocytes (T-LGL) can be observed in different situations such as viral infections, autoimmune diseases, paraneoplastic conditions, and following allogeneic hematopoietic stem cell transplantation (HSCT); in the latter condition as a sign of immune system reconstitution. Some of these expansions have been described as clonal T-LGL proliferations.

Aim of the study: We performed a retrospective analysis to evaluate the frequency, cellular phenotype, clonality, clinical features and evolution of persistent post-transplant T-LGL in a single center cohort of patients, who underwent an allogeneic HSCT.

Materials and methods: Peripheral blood counts and lymphocyte subpopulations were systematically revised in all yearly HSCT post-transplant control examinations performed between 1st June and 31st December 2009. In all cases with persistent lymphocytosis (defined as $>3 \mathrm{G} / 1$ for at least 3 months) and with a pathologic CD4/CD8-ratio of Tlymphocytes, defined as CD4/CD8 $<1.0$ or $>1.5$, additional 
flow cytometry investigations were done to establish the diagnosis of a T-LGL expansion. Further, T-cell receptor Gamma (TCR-Gamma)-gene fragment lengths were analyzed after PCR-amplification by ABI 310 Genetic Analyzer to assess Tcell clonality; primers to all existing $\mathrm{V}$ and $\mathrm{J}$ genes, except the V1S1P gene, whose recombination signal sequences with a spacer sequence of only $11 \mathrm{bp}$ will not participate in a rearrangement, were applied. Patients with T-LGL expansion were compared to the sub-cohort of patients without T-LGL expansion, using the chi-square test for categorical data and the Mann-Whitney U test for continuous variables. To identify independent prognostic risk factors, a multivariate stepwise linear regression analysis was performed.

Results: Within 215 evaluated recipients, 14 (7\%) were diagnosed with T-LGL expansions; 7/14 patients were females, the median age was 39 years (range 25-64). The median lymphocyte count was 4.24 G/1 (3.0-26.5). The median time between HSCT to first diagnosis of TLGL lymphocytosis was 16 months (range 3-58). The median duration of lymphocytosis persistence was 31 months (3-179). 13/14 patients were in complete remission of their underlying disease showing a $100 \%$ donor chimerism, 1/14 patient had a stable disease after graft rejection. Neutrophil counts were normal in all patients with T-LGL expansion. 8/14 patients (57\%) showed a mild hyporegenerative anemia. Thrombocytopenia was present in $2 / 14$ patients (14\%). No autoimmune manifestations were observed in this group. 8/14 patients were on immunosuppressive therapy, of whom 6/8 had signs of graft versus host disease (GvHD). In univariate analysis, patients with T-LGL expansion presented more often with a history of Cytomegalovirus $(\mathrm{CMV})$ reactivation $(p=0.001)$ and of $\operatorname{GvHD}(p=0.02)$, when compared to patients without T-LGL proliferations. In multivariate analysis, CMV reactivation [Relative Risk (RR) 5.063; $95 \%$ CI: 1.586-16.160; $p=0.006$ ], but not GvHD (RR 2.831; 95 \%CI: 0.831-9.648; $p=$ 0.096) showed a significant association with T-LGL expansion. Patients' age, conditioning regimen, donor type, graft source, initial diagnosis as well as relapse after HSCT were not related to T-LGL expansion. Immunophenotyping showed CD3/CD8-positivity in all cases except for one patient, who showed a CD3/CD4 phenotype. There was no CD5 antigen loss. TCRGamma fragment length analysis revealed polyclonal expansions in 9/14 and clonal T-cells in 5/14 patients.

Conclusions: Persistent lymphocytosis with T-LGL expansion seen after allogeneic HSCT is polyclonal in most cases but can also be clonal like in patients with T-LGL-leukemia, yet not showing an antigenic loss. Despite the long duration of such expansions, the majority of cases have an indolent clinical course without symptoms of T-LGL leukemia. Our data further point that such T-LGL proliferations most probably reflect a chronic immunologic stimulation triggered by CMV reactivation or, more rarely, by GvHD.

\section{BMS003}

\section{Platelet derived growth factor receptor - an early marker for myelofibrosis}

Judit Bedekovics ${ }^{1}$; Attila Kiss ${ }^{1}$; Katalin Károlyi ${ }^{1}$; Gábor Méhes ${ }^{1}$

(1) Department of Pathology, University of Debrecen, Hungary

Background: The platelet derived growth factor receptor (PDGFR) activation induces fibroblast proliferation in fibrotic conditions such as scar formation, liver, kidney or lung fibrosis and scleroderma. PDGF receptors consist of two subunits (PDGFR $\alpha$ and PDGFR $\beta$ ) which can compose homo- or heterodimers. PDGF receptors belong to the tyrosine kinase receptor family. There are differences between the receptor subunits what the mesenchymal cells express which can lead to different response to PDGF stimuli.

Aim of the study: The aim of this study was to evaluate the distribution of PDGFR $\alpha$ and $\beta$ subunits in non-fibrotic bone marrow (BM). Further our purpose was to assess the correlation between the level of PDGFR expression and the grade of myelofibrosis (MF grade).

Materials and methods: PDGFR expression in nonhemopoietic mesenchymal cells was determined in 89 bone marrow biopsies by immunohistochemical stainings using primary antibodies against PDGFR $\alpha$, PDGFR $\beta$, CD 34, SMA. In selected cases double staining were performed. A four grade (0-3) semi-quantitative scoring system was created to evaluate the level of PDGFR expression. The grade of myelofibrosis (MF) was evaluated using reticulin silver staining.

Results: In both non-fibrotic and fibrotic BM PDGFR $\alpha$ and PDGFR $\beta$ subunits were found to be differentially expressed. Activated fibroblasts were specifically highlighted by PDGFR $\beta$ expression while PDGFR $\alpha$ identified endothelial cells, endosteal cells and only a small subfraction of stromal fibroblasts.

In the non-fibrotic control BM there were no significant differences between the PDGFR $\beta$ expression level and myelofibrosis grade $(p=0.15)$ while in the fibrotic group the PDGFR $\beta$ expression level was significantly higher $(p=$ $0.014)$. However there was good correlation between the grading systems $(r 2=0.74)$, in some of the early changes the PDGFR expression exceeded the level of fibrosis determined by reticulin silver staining.

Conclusions: In conclusion, PDGFR $\beta$ expression in the bone marrow is closely related to the grade of MF, but 
non-fibrotic (prefibrotic) samples could also be identified. Using a four grade (0-3) scoring system for the determination of PDGFR $\beta$ positivity following IHC, a new, functional classification of the bone marrow stromal reaction in different hematological conditions can be established.

\section{BMS004}

P53 immunoreactivity in bone marrow biopsies of patients with myelodysplastic syndromes correlates with overall and leukemia-free survival

Emanuela Boveri ${ }^{1}$; Giorgio A Croci ${ }^{2}$; Matteo G Della Porta $^{2}$; Luca Malcovati ${ }^{2}$; Marcello Gambacorta ${ }^{3}$; Umberto Gianelli ${ }^{4}$; Giuseppe Isimbaldi ${ }^{5}$; Alessia Moro $^{6}$; Maurilio Ponzoni $^{7}$; Marco Ungari ${ }^{8}$; Claudio Schena ${ }^{2}$; Marco Lucioni $^{1}$; Mario Cazzola ${ }^{9}$; Marco Paulli ${ }^{10}$

(1) Anatomic Pathology, Foundation IRCCS Policlinico San Matteo, Pavia (2) Institute of Surgical Pathology, Fondazione IRCCS Policlinico San Matteo, University of Pavia (3) Surgical Pathology, Ospedale Niguarda-Ca' Granda (4) Institute of Surgical Pathology, University of Milano, Fondazione IRCCS Ca' Granda Ospedale Maggiore (5) Surgical Pathology, Policlinico di Monza (6) Surgical Pathology, Ospedale San Paolo (7) Institute of Surgical Pathology, Ospedale San Raffaele (8) Institute of Surgical Pathology, Spedali Civili di Brescia, University of Brescia (9) Division of Hematology, Fondazione IRCCS Policlinico San Matteo, University of Pavia (10) Anatomic Pathology Section, University of Pavia

Background: The role of p53 expression, as a result of different activation pathways or mutations of TP53, has been recently underlined in myelodysplastic syndromes (MDS).

Aim of the study: to correlate the value of p53 immunoreactivity (IR) on bone marrow biopsies (BMBs) with clinical and histopathological data and outcome in a large series of patients with MDS.

Materials and methods: We assessed p53-IR as the percentage of hematopoietic cells showing intense nuclear staining (p53++), on the BMBs at diagnosis from 270 MDS patients (refractory cytopenia with unilineage dysplasia: 51 , refractory anemia with ring sideroblasts: 39 , refractory cytopenia with multilineage dysplasia: 84 , MDS with isolated del $(5 \mathrm{q})$ : 18 , refractory anemia with excess of blasts-RAEB type 1: 41, RAEB type 2: 37; IPSS: Low: 105, Int-1: 108, Int-2: 46, High: 11). A cut-off value of $2 \%$ was used.

Results: Median percentage of p53++ cells was $0.32 \%$ (range 0-69\%) on the entire series; 48 patients (18\%) showed $\mathrm{p} 53-\mathrm{IR}>2 \%$ (median value $4 \%$, range $2-69 \%$ ).
Univariate analysis demonstrated significant correlations between p53-IR and multilineage dysplasia $(p=.045)$, excess of blasts $(p=.026)$, poor risk cytogenetics $(p=.012)$, transfusion dependency $(p=.013)$, and previous exposure to radiation or chemotherapy $(p=.016)$; among histological parameters, $\mathrm{p} 53-\mathrm{IR}>2 \%$ was associated with the presence of CD34+ cell clusters $(p=.016)$ and not with fibrosis degree $(p=.86)$.

Evolution to acute leukemia occurred in $88 / 270$ cases (33\%): 55/222 (25\%) patients showed p53-IR $<2 \%$ and $33 / 48(69 \%)$ p53-IR $>2 \%(p<.001)$. Overall survival (OS) and leukemia free survival (LFS) correlated with p53-IR in the whole series $(p<.001)$ and, notably, also in the subgroup without excess of blasts (OS: $p=.009$; LFS: $p=.023$ ).

At multivariate analysis with age, sex and IPSS, p53-IR> $2 \%$ negatively correlated with OS $(\mathrm{HR}=2.39, p<.001)$ and LFS $(\mathrm{HR}=3.08, p<.001)$ in the whole series and in patients without excess of blasts (OS: HR=2.65, $p=.01$; LFS: HR= $3.31, p=.003$ ). Results were similar considering WPSS instead of IPSS.

Conclusion: p53-IR on BMBs reveals an independent parameter for the prognostic assessment of MDS and can be integrated in the histopathological work-up of these patients.

\section{BMS005}

In non-follicular lymphoproliferative disorders, IG/BCL 2-fusion is not restricted to chronic lymphocytic leukemia

Lucile Baseggio ${ }^{1}$; Marie-Odile Geay ${ }^{2}$; Sophie Gazzo ${ }^{2}$; Françoise Berger ${ }^{3}$; Alexandra Traverse-Glehen ${ }^{3}$; Martine Ffrench $^{2}$; Sandrine Hayette ${ }^{2}$; Evelyne Callet-Bauchu ${ }^{2}$; Aurélie Verney $^{2}$; Dominique Morel$^{2}$; Laurent Jallades ${ }^{2}$; Jean-Pierre Magaud $^{2}$; Gilles Salles ${ }^{1}$; Pascale Felman ${ }^{2}$

(1) CHU lyon-sud/HCL (2) Laboratoire d'Hématologie (3) Service d'anatomie pathologique, Lyon, France

Background: The translocation $t(14 ; 18)$ and its variants $t$ $(2 ; 18)$ and $t(18,22)$, involving BCL2, the genetic hallmark of follicular lymphoma (FL), have been also reported in few cases of chronic B-cell lymphoproliferative disease (CLPD) and mainly in chronic lymphocytic leukaemia (CLL).

Materials and methods: Herein we described the clinical, morphological, immunological, cytogenetic and molecular findings in 37 cases of $t(14 ; 18)$-positive CLPD, identified from our series of non-FL B-cell neoplasms $(n=993)$ routinely analysed in peripheral blood by conventional cytogenetics. The diagnosis of FL was excluded by morphology and immunology (CD10 negativity in all cases).

Results: These BCL2 translocations were observed in 22 CLL cases including 7 monoclonal B-cell lymphocytosis 
(MBL) re-classified according to new IWCLL criteria, in 6 small lymphocytic lymphomas (SLL), but also in the first splenic marginal zone lymphoma (SMZL) and 8 unclassifiable CLPD with overlapping CLL/MZL features. In CLL cases, IG/BCL2-fusion was remarkably associated to trisomy $12(13 / 22)$, mutated IGHV status (20/21) and did not affect the outcome. Moreover most of these CLLs harbour a low mutation load of BCL6 gene and unmutated CD95 loci which points to a post germinal centre cellular origin.

Conclusions: Here we confirm that IG/BCL2 fusion is a rare (3\%) but recurrent cytogenetic abnormality in CLL. In addition this anomaly appears to be not restricted to CLL since we report beside a heterogeneous group of borderline CLL/MZL cases, the first SMZL bearing IG/BCL2 fusion.

\section{BMS006}

\section{Primary splenic angiosarcoma revealed by bone marrow metastasis}

Marouan Sofia ${ }^{1}$; Asmaa Quessar $^{1}$; Soumya Zamiati ${ }^{1}$; Said Benchekroun $^{1}$

\section{(1) CHU ibn rochd, Casablanca, Morocco}

Background: Bone marrow (BM) is one of the common sites to be involved by solid tumors that metastasize via bloodstream. This condition affects the prognosis and management of these tumors. The incidence of BM involvement varies with types of primary tumors. The solid tumors most frequently detected in BM in adults are carcinoma of the breast, prostate, lung and gastrointestinal tract. In children, it is rather neuroblastoma. Diffuse marrow replacement by sarcomatous cells is rare in adults and angiosarcoma replacing BM is exceedingly rare. We report here the pathologic findings of a Moroccan patient with BM metastasis of a primary unsuspected splenic angiosarcoma.

Results: A 26 year-old Moroccan man presented with a deep anemia and a splenomegaly without enlargement of lymph nodes or other symptoms. Bone marrow biopsy was performed and histopathologic exam revealed replacement of marrow spaces by proliferating spindle cells with moderate atypia and a few mitotic figures. There were tumor cells forming vascular channels. The immunohistochemical study showed that the tumor cells were positive for CD34 and negative for CD31 without expression of HHV8. These findings were highly suspicious for BM involvement by angiosarcoma. Contrast enhanced CT showed enlarged spleen with multiple heterogenous lesions. We proceeded with splenectomy and postoperative histopathological examination of the specimen revealed an authentic angiosarcoma of the spleen.
Conclusions: Few reports in the literature describe angiosarcomatosis of the BM. It is important to recognize this unusual presentation to avoid confusion with other more common BM replacing entities and to adapt an efficient treatment. Splenic angiosarcoma can metastasize to the bone marrow and anemia may be an indicator of the advanced disease. In the differential diagnosis of the anemia, bone marrow metastasis and BM biopsy should be considered in suspected cases. Synchronous BM metastasis from primary splenic angiosarcoma is even rarer with only two prior descriptions in the literature.

\section{BMS007}

Regulatory $T$ cells (Tregs) in the background of $T /$ NK cell large granular lymphocytic leukemia (LGLL)

Filiz Sen ${ }^{1}$; Judith Brody ${ }^{2}$; Xinmin Zhang ${ }^{2}$; Steven Allen ${ }^{2}$; Jonathan Kolitz ${ }^{2}$; Joseph Ramek ${ }^{2}$; Crina Vintila ${ }^{2}$; Jordan Laser $^{3}$; Howard Ratech ${ }^{4}$

(1) North Shore-LIJ Health System, NY, USA (2) NSHS-LIJ (3) NS-LIJ (4) Albert Einstein College of Medicine/Montefiore Medical Center

Background: Regulatory T cells (Tregs) is a CD4 positive $\mathrm{T}$ cell subset that functions to prevent autoimmunity and to regulate physiologic immune reactions. Tregs are also present in the tumor microenvironment and appear to play an important role in the pathophysiology of malignant processes. Available data suggests that this role is contextdependent, as a higher density of tumor infiltrating Tregs at diagnosis may be associated with either a positive or a negative clinical outcome. Positive associations have been reported in various lymphomas, most prominently B cell lymphomas of germinal center origin.

Aim of the study: To investigate the density of the regulatory $\mathrm{T}$ cells in large granular lymphocytic leukemia (LGLL) and identify clinicopathological associations.

Materials and methods: We performed immunohistochemical studies for Foxp3 using the paraffin embedded sections of bone marrow core biopsies from seven patients with $\mathrm{T} /$ NK cell large granular lymphocytic leukemia. The immunostained slides for Foxp3 were analyzed and scored by two investigators (JB and $\mathrm{XZ}$ ) in a blind fashion. Additional immunohistochemical studies for CD2, CD3, CD4, CD5, CD7, CD8, CD56, CD57, TIA-1 and GranzymeB were performed. Molecular genetic studies for TCR gene rearrangement were performed. A control group of three marrow core biopsies from patients with no diagnostic abnormalities was used.

Results: All cases showed either an interstitial or interstitial and intrasinusoidal marrow involvement. All cases showed 
an atypical T cell or NK cell phenotype by flow cytometry analysis. Overall, Foxp 3 density ranged from 0.0 to 8.4 cells per high power field (median $0.25 / \mathrm{hpf}$ ). In contrast, Foxp3 density in the control group of three cases was 0, 0.09 and $0.2 / \mathrm{hpf}$ (median $0.09 / \mathrm{hpf}$ ). One case of NK cell LGLL showed the highest Foxp3 density. Six out of seven cases showed clonal $\mathrm{T}$ cell receptor gene rearrangements.

Conclusions: In this pilot study group, we identified differences in Foxp3 density in T/NK cell LGLL versus normal marrow biopsies. In addition, it appears that there is a difference between NK cell versus T cell LGL. These findings need to be confirmed and further investigated by larger case series.

\section{BMS008}

\section{"Which Came First, The T-PLL Or The Myeloma?" A Case Report}

Nikolay Stoyanov'; Tihomir Dikov²; Georgi Momekov²; Vera Stoeva $^{1}$; Georgui Balatzenko ${ }^{1}$; Margarita Guenova ${ }^{1}$

\section{(1) NSBALHZ (2) Medical University Sofia Bulgaria}

Background: We present the diagnostic findings from a patient who presented with skin lesion suspicious for melanoma and was subsequently found to have plasma cell myeloma (PCM) and T-prolymphocytic leukemia (T-PLL) within a month from initial examination.

Materials, methods, and results: A 48-year-old man was admitted to our hospital with a history of a bleeding blackcoloured skin lesion in the shoulder region and a fastgrowing mass in the sternal region. Clinical findings were considered suggestive of metastatic melanoma. No enlarged lymph nodes or hepatosplenomegaly were present on clinical examination or ultrasonography. Radiological studies revealed retrosternal oval tumor mass measuring $4 \times 7 \mathrm{~cm}$, infiltrating subcutaneous fat tissue and muscles, and protruding into the thoracic cavity. Skin and sternal tumour biopsies were reviewed to reveal a benign naevomelanocytic proliferation and a plasma cell tumour. Blood tests showed normal haemoglobin levels $(152 \mathrm{~g} / \mathrm{L})$, platelet counts $(226 \mathrm{G} / \mathrm{L})$ and surprising leukocytosis $(26 \mathrm{G} / \mathrm{L})$. Manual differential showed $75 \%$ atypical, matureappearing lymphocytes with cytoplasmic blebs. Plasma IgG kappa measured 36, $49 \mathrm{~g} / \mathrm{L}$. Laboratory chemistry investigations were within reference ranges. Virological tests for HIV, EBV, HBV, and HCV were all negative. Bone marrow aspirate smear was hypercellular with $40 \%$ lymphocytes having identical appearance and $11 \%$ plasma cells. Granulocytic, erythroid and megakaryocytic lineages although reduced were maturing normally. Blood flow cytometry studies confirmed lymphocytosis $(10.2 \mathrm{G} / \mathrm{L})$ with the majority of lymphocytes $(8.8 \mathrm{G} / \mathrm{L})$ having mature aberrant T-cell phenotype: $\mathrm{CD} 2+\mathrm{CD} 3+\mathrm{CD} 5+\mathrm{CD} 7+\mathrm{TCR} \alpha \beta+$, and with $\mathrm{CD} 4+$ and $\mathrm{CD} 8+$ co-expression. Bone marrow FCM evaluation showed $2 \%$ CD138+ CD19- CD56+ abnormal plasma cells. Bone marrow biopsy showed hypercellularity with $15 \% \mathrm{CD} 138+$ plasma cells. Normal hematopoiesis was preserved; a small amount of scattered CD3+ positive T-cells were present.

A diagnosis of two primary neoplasias was established: Tcell prolymphocytic leukemia and plasma cell myeloma. After 2 years of treatment with alternating CHOP and VAD protocols and local radiotherapy for the parasternal tumour mass the patient was readmitted in a worsened state. Blood tests revealed anaemia $(\mathrm{Hb} 107 \mathrm{~g} / \mathrm{L})$, low platelets (32 G/L) and extreme leukocytosis (426 G/L). Blood tests showed $99 \%$ atypical lymphocytes bearing the initial immunophenotype. Marrow biopsy was packed with T-cells $(\mathrm{CD} 3+\mathrm{CD} 5+45 \mathrm{RO}+)$ and $\mathrm{CD} 138+$ plasma cells (about $25 \%$ ). At that time patient's condition worsened progressively and the patient died of infectious complications.

Conclusions: T-PLL is a rare aggressive disease and only rarely reported in association with other malignancies. The case presented is unusual for at least two reasons. Firstly, TPLL followed a relatively indolent course with subtle symptoms and gradually rising lymphocytosis. Secondly, the concurrent plasma cell myeloma posed both diagnostic and therapeutic challenges. It remains elusive whether these two malignancies share common genetic abnormalities. Moreover, each of them could pave the road for the other via disrupting major mechanisms of anti-tumour immunity. It is, however, evident that these two entities are not mutually exclusive and any suspicion for a second malignancy grants respective work-up.

This work was supported by The Bulgarian National Science Fund.

\section{BMS009}

Immunophenotype and morphological analysis of leukemia/ lymphoblastic lymphoma in adults

\section{Alfredo Valero Gómez ${ }^{1}$}

\section{(1) Hospital General de México, Mexico City, Mexico}

Background: Leukemia/lymphoblastic lymphoma (LLL) is a neoplasm of lymphoid blasts; $80 \%$ are originated in B cells (LLB) and the remnant $20 \%$ from T cells. Cells are small or mid-sized. In adults, the mean age of presentation is 40 years. The lack of CD10 expression in blasts has been associated with translocation of mixed lineage leukemia (11q23) and adverse prognosis. 
Aim of the study: To analyze immunophenotype, morphology, and prognosis.

Materials and methods: Fourteen patients with diagnosis of LLL from 2007 to 2011 were included. We analyzed the following variables: gender, age ( 25 to 55 years), clinical presentation, location, morphology, immunophenotype $\mathrm{B} / \mathrm{T}$ with the following markers: CD 20, CD $79 \mathrm{a}$, TdT, CD10, CD 117, CD 99, CD 34, CD2, and CD 3.

Results: The same frequency was observed for both genders, mean age was 43.2 years, $71.4 \%$ of the patients were affected by leukemia, $85.7 \%$ were immunophenotype B, $71.4 \%$ showed morphologically monotone blasts and in two patients (14.2\%) lack of CD10 expression was observed and unusual morphology such as pleomorphism and "starrysky pattern" with adverse clinical course.

Conclusions: Our data suggest that the pleomorphic morphology and lack of CD10 expression in precursor lymphoid neoplasms are rare in adults $(14.2 \%)$, but they correlate with data suggestive of poor clinical prognosis. Prospective studies are needed to confirm this observation and characterize better high risk CD10 negative subgroups in precursor lymphoid neoplasms.

\section{$\underline{\text { BMS010 }}$}

\section{A Case of Non-leukemic Myeloid Sarcoma with FIP1L1- PDGFRA Rearrangement: An Unusual Presentation of a Rare Disease}

Dong Chen ${ }^{1}$; Veronika Bachanova ${ }^{2}$; Rhett P. Ketterling ${ }^{3}$; Kebede H. Begna ${ }^{3}$; Curtis A. Hanson ${ }^{3}$; David S. Viswanatha ${ }^{3}$

\section{(1) Mayo Clinic (2) University of Minnesota (3) Mayo Clinic, Rochester, MN, USA}

Background: The FIP1L1-PDGFRA gene rearrangement defines a distinct hematopoietic neoplasm which commonly presents with sustained eosinophilia and is highly sensitive to imatinib mesylate treatment. Although acute myeloid or lymphoblastic leukemias with such a rearrangement can occur, non-leukemic myeloid sarcoma has not been reported and its sensitivity to imatinib treatment is unknown. Here we report such a case.

Design: A 31-year-old man was incidentally found to have marked eosinophilia $\left(31.5 \times 10^{9} / \mathrm{L}\right)$ and an infiltrative mass $(4.6 \mathrm{~cm})$ within the right shoulder subcutaneous soft tissue. A bone marrow aspirate and biopsy, and an incisional biopsy of the mass were performed. Peripheral blood and bone marrow aspirate smears, and bone marrow and soft tissue mass biopsy slides were examined. Immunohistochemical (IHC) stains using antibodies to CD25, CD34, CD117, myeloproxidase and tryptase were performed on both bone marrow and soft tissue biopsy sections. Cytogenetic studies including
FISH analyses for BCR-ABL, MYH11-CBFb, CHIC2 deletion (FIP1L1-PDGFRA) were performed on bone marrow aspirate cells. The CHIC2 deletion FISH study was also performed on sections of the mass biopsy specimen. KIT D816V mutation analysis was performed on bone marrow cells.

Results: Except for the marked eosinophilia and rare spindleshaped, CD25-positive bone marrow mast cells, his peripheral blood and bone marrow showed no features of an acute leukemia, myelodysplasia or lymphoma. Karyotyping studies of bone marrow aspirate was normal, but $67 \%$ nuclei were positive for FIP1L1-PDGFRA rearrangement by FISH. The remaining FISH studies and KIT D816V mutation analysis were all negative. The soft tissue mass showed significant infiltration by blasts that co-expressed CD34, CD117 and myeloproxidase by IHC stains. Approximately $70 \%$ of blasts were positive for FIP1L1-PDGFRA rearrangement by FISH. A diagnosis of non-leukemic myeloid sarcoma with FIP1L1PDGFRA rearrangement was made. The patient consequently received daily $100 \mathrm{mg}$ imatinib mesylate treatment. His eosinophilia and myeloid sarcoma completely resolved 14 days and 3 months after treatment, respectively; and he also archived complete hematologic and cytogenetic remission.

Conclusions: To our knowledge, this is the first case of an non-leukemic myeloid sarcoma with the FIP1L1-PDGFRA rearrangement. Despite the aggressive clinical behavior attributed to myeloid sarcoma generally, the presence of FIP1L1-PDGFRA rearrangement in this case still retains high sensitivity to imatinib treatment.

\section{BMS011}

Ferritin $\mathbf{H}$ is a maker of erythroid precursors in bone marrow biopsy specimens

Wei Wang ${ }^{1}$; Suzy Torti ${ }^{1}$; Frank Torti ${ }^{1}$; Michael Beaty ${ }^{1}$

(1)Wake Forest Baptist Health, Winston-Salem, NC, USA

Background: Accurately determining erythroid precursors can be challenging in bone marrow biopsy specimens, especially in the condition of dyserythropoiesis. The traditional markers of erythroid lineage are glycophorin A and hemoglobin A. Both proteins, however, are expressed at high levels in mature erythrocytes and are not reliable for early erythroid precursors. CD71, an iron uptake protein, has been recently identified as a useful marker in immunohistochemical studies of erythroid precursors, but it also serves as a proliferation marker and is expressed in other cells with high mitotic rates. Iron is important for haem synthesis. Before hemoglobin synthesis, iron is stored in ferritin, an iron storage protein consisting of $\mathrm{H}$ and $\mathrm{L}$ subunits. As erythroid cells start to synthesize haem at the basophilic erythroblast stage, iron is released from ferritin and incorporated into haem. Iron storage and iron release are 
associated with ferritin protein synthesis and degradation, respectively. Thus, ferritin has its highest level in the earliest erythroid precursors and decreases exponentially with hemoglobulin synthesis and erythroid maturation. Additional studies also demonstrated that erythroblasts contain much higher levels of ferritin than the cells of other hematopoietic lineages. Based on these, we proposed that ferritin can be used as a specific marker of erythroid precursors in bone marrow specimens.

Aim of the study: To determine whether ferritin is a specific and reliable marker for erythroid precursors in bone marrow biopsy specimens.

Materials and methods: Immunohistochemistry was performed using a monoclonal antibody against ferritin $\mathrm{H}$. Thirty cases used in this study included normal bone marrows, acute myeloid leukemia (subtypes from M0 to M7), bone marrows involved by plasma cell myeloma, Burkitt lymphoma, chronic lymphocytic lymphoma, diffuse large B cell lymphoma, and follicular lymphoma.

Results: In normal bone marrow, nucleated erythroid precursors are positive, while mature erythrocytes are completely negative. Myeloid cells, lymphoid cells and megakaryocytes at all stages show no expression of ferritin H. Erythroid blasts in acute erythroid leukemia (M6) are strongly positive for ferritin $\mathrm{H}(5 / 5)$, while blast cells from all other subtypes of leukaemia show negative immunostaining. In bone marrows involved by plasma cell myeloma and various lymphomas, ferritin $\mathrm{H}$ is negative in neoplastic plasma cells and lymphocytes. It is noteworthy to mention that macrophages in bone marrow are positive for ferritin $\mathrm{H}$, but they can be easily distinguished from erythroid cells based on morphological difference.

Conclusions: Ferritin $\mathrm{H}$ is a novel and reliable marker of erythroid precursors and can be used in immunohistochemistry to identify cells of the erythroid lineage in bone marrow biopsy specimens.

\section{BMS012}

\section{FISH analysis of multiple myeloma using paraffin embedded sections}

\section{Tomoki Kikuchi}

Department of Pathology, Tokai University School of Medicine, Japan

Background: Cytogenetic abnormality of multiple myeloma (MM) has not been well investigated so far because of low proliferating rate of the neoplastic cells of MM. Fluorescence in situ hybridization (FISH) is a useful tool for detecting cytogenetic abnormalities of MM by evaluating the non-proliferating (interphase) neoplastic cells, although there is difficulty in handling paraffin embedded tissue.
Aim of the study: To disclose cytogenetic abnormality of MM in Japanese population.

Materials and methods: We investigated cytogenetic abnormalities of IGH/FGFR3 fusion, MYC break apart, 17p13.1 deletion, 13q14.3 deletion, 5p15.2/9p11-q11 hyperploidy and CCND1 breakapart in 28 cases of MM by FISH using the paraffin embedded section of bone marrow clot. Slides with cell suspension made by a $100 \mu \mathrm{m}$-thick section were used. More than $10 \%$ of abnormal signals were evaluated as positive. Results: Cytogenetic abnormalities were detected: IGH/ FGFR3, 4/27 (14.8 \%); MYC, 4/26(15.4\%); 17p13.1 4/26 (15.4\%); 13q14.3, 0/25; hyperploidy, 6/21 (28.6\%); CCND1, 4/28 (14.3\%). Six cases were negative for all 6 probes examined $(6 / 20,30 \%)$.

Conclusions: Various cytogenetic abnormalities were found in MM. IGH/FGFR3 and six-negative may be related to early death and good prognosis, respectively.

\section{BMS013}

\section{Increased bone marrow angiogenesis in graft-versus- host disease}

Michael Medinger ${ }^{1}$; Christoph Bucher ${ }^{1}$; Jörg Halter ${ }^{1}$; Alois Gratwohl $^{1}$; Jakob Passweg ${ }^{1}$; Alicia Rovo ${ }^{1}$; Stephan Dirnhofer $^{2}$; Andre Tichelli ${ }^{1}$; Alexander Tzankov ${ }^{2}$

(1) Hematology, University Hospital Basel (2) Institute of Pathology, University Hospital Basel, Switzerland

Background: Allogeneic hematopoietic stem cell transplantation (HSCT) is often the only curative approach for patients with hematological malignancies. The success of HSCT is severely challenged by graft-versus-host disease (GvHD) mediated by host-reactive, donor-derived T cells. There is increasing evidence that both angiogenesis and endothelial injury are involved in GvHD.

Aim of the study: We examined bone marrow microvessel density (MVD) and Vascular Endothelial Growth Factor/Receptor (VEGF/VEGFR) expression, in comparison to normal controls, in patients treated with HSCT for acute myelogenous leukemia (AML) and correlated the results with the presence and degree of acute and chronic GvHD (aGvHD, cGVHD). Further, in sex-mismatched transplantations, we examined the source of endothelial cells with XY fluorescence in situ hybridization (FISH) probes assessing endothelial cell chimerism.

Materials and methods: All 26 study patients had an AML and underwent HSCT at our institution between 2004 and 2010 and fulfilled following criteria: (1) protocol bone marrow biopsies 1 year after HSCT were available; and (2) AML was in complete remission. The samples were immunohistochemically stained for CD34, CD105, VE-cadherin, VEGF, VEGFR-1 and VEGFR-2. MVD was assessed by 
counting the number of CD34-, CD105- and VE-cadherinpositive vessels in $6.65 \mathrm{~mm} 2$ at $200 \times$ magnification. Expression of VEGF and VEGFR was assessed by counting the number of positive cells in $0.85 \mathrm{~mm} 2$ at $400 \times$ magnification. Further, the percentage of VEGF-positive megakaryocytes was assessed. FISH was performed on the bone marrow biopsy specimen using a directly labeled, dual color $\mathrm{X}$ - and Y-chromosome probe mix (Xp11.1-q11.1/Yq12). 100 endothelial cells and 2000 hematopoietic cells were analyzed. Age-matched controls for immunohistochemical studies consisted of 20 non-transplanted patients without evidence of bone marrow abnormalities. Comparisons were performed with Kruskal-Wallis- and Mann-Whitney Utests. The Spearman rank correlation coefficient and Rsquare were applied, where appropriate.

Results: Of the 26 patients (median age 47 years, range 24 66 ) included in the study, 14 were male and 12 female. All received peripheral blood stem cells as stem cell source with matched sibling donors $(i=19)$ or unrelated donors $(n=7)$. The median interval to bone marrow biopsy was 371 days (range 350-385) after HSCT and hematopoietic donorchimerism at that time point was $100 \%$ in all patients. The median interval from HSCT to clinical GvHD onset of any grade was 21 days (range 7-146). The GvHD type was classic acute in 13 patients and late-onset acute in 2 patients, the other 11 did not suffer from aGvHD. Of the 15 patients with aGvHD, 7 progressed to moderate or severe cGvHD, while 4 of the 11 latter developed moderate to severe cGvHD. In patients with previous aGvHD, we found a significantly higher CD105-MVD $(8.6 \pm 2.4 / \mathrm{mm} 2$ vs. $6.9 \pm$ $3.4 / \mathrm{mm} 2, p=0.026)$ and VE-cadherin-MVD $(10.0 \pm 1.7 / \mathrm{mm} 2$ vs. $5.9 \pm 2.2 / \mathrm{mm} 2, p=0.0004)$, but not CD34-MVD, than in patients without aGvHD or controls $(p=0.028$ and 0.0001 , respectively). In patients with cGvHD, we found minimally decreased CD34-MVD and no differences in CD105-MVD and VE-cadherin-MVD compared to patients without cGvHD, the VE-cadherin-MVD being still higher than in controls $(8.5 \pm$ $2.7 / \mathrm{mm} 2$ vs. $5.3 \pm 2.0 / \mathrm{mm} 2, p=0.027$ ). In aGvHD, we also found more VEGF+ megakaryocytes than in patients without aGvHD and in controls $(92 \pm 9 \%$ of all megakaryocytes vs. 52 $\pm 20 \%$ vs. $58 \pm 27 \%, p=0.00007$ ). MVD assessed by CD 105 and VE-cadherin correlated with the relative number of VEGFpositive cells (Spearman $\rho=0.484, P=0.017$, R-square $=0.125$ and $\rho=0.606, P=0.002$, R-square $=0.506$ respectively). In five male patients grafted from a female donor XY FISH revealed a persistence of recipient-type XY chromosome karyotype in most bone marrow endothelial cells and all osteocytes after HSCT. Single donor-type XX endothelial cells without Y signals were rarely observable ( 3 of 5 patients), whereas almost all nucleated marrow cells were donor type. In one patient a higher amount of donor-derived endothelial cells was observed (approximately $10 \%$ ). This was the only patient who lacked a HLA-identical sibling or a matched unrelated donor and therefore received a haploidentical, $\mathrm{T}$ cell-depleted graft from his mother.

Conclusions: Taken together, MVD by newly formed CD105- and/or VE-cadherin-positive vessels and VEGF expression are increased in aGvHD and to a minor extend in cGvHD as compared to normal bone marrow. Donor-derived endothelial cells play, if at all, a minor role in neovascularization and endothelial replacement. Whether neovascularization simply reflects a secondary reactive process due to inflammation or is a GvHD-specific process is currently unknown. If the latter is true anti-angiogenic therapies could be potentially useful in the prevention or therapy of GvHD.

\section{BMS014}

Clonal expansion of a reactive CD8+CD57+ large granular lymphocyte population in the bone marrow after autologous stem cell transplant in myeloma

Kristy Wolniak ${ }^{1}$; Charles L. Goolsby ${ }^{2}$; Anjen Chenn ${ }^{2}$; Seema Singhal ${ }^{3}$; Jayesh Mehta ${ }^{3}$; David Dittman ${ }^{2}$; Jennifer Scalise $^{4}$; LoAnn C. Peterson ${ }^{4}$

(1) Northwestern University Feinberg School of Medicine (2) Department of Pathology, Northwestern University Feinberg School of Medicine and Robert H. Lurie Co (3) Department of Medicine Northwestern University Feinberg School of Medicine and Robert H. Lurie Compr (4) Department of Pathology, Northwestern University Feinberg School of Medicine, Chicago, IL, USA

Background: Clonal expansions of T-large granular lymphocytes (T-LGLs) have been identified in patients following allogeneic and autologous stem cell transplants, and may represent either post-transplant LGL leukemias or reactive immune responses. Two studies have reported LGL leukemia after autologous transplant. Autologous stem cell transplant is a mainstay of myeloma therapy, and the implications of a neoplastic expansion versus a reactive immune expansion of LGLs following autologous transplant in myeloma patients are strikingly different.

Aim of the study: To differentiate between reactive and neoplastic T-LGL expansions, we assessed peripheral blood and bone marrow of 20 myeloma patients $45-90$ days postautologous stem cell transplant, 9 myeloma patients without transplant, 9 newly diagnosed myeloma patients, 7 patients with monoclonal gammopathy of unknown significance, 6 myeloma patients greater that 6 months post-transplant, and 8 controls.

Materials and methods: Comprehensive flow cytometric immunophenotyping and molecular studies for T cell receptor (TCR) clonality were performed on the bone marrows and clinical records reviewed. 
Results: All myeloma patients examined after autologous stem cell transplant had a significant increase in the LGLs in the peripheral blood and in the percent bone marrow CD8+ cells of CD3+ lymphocytes (71\%) as compared to controls (39\%). The LGLs had a reproducible immunophenotype of CD8+CD57+ cytotoxic T-cells without phenotypic abnormalities in 19/20 patients. Surprisingly, patients up to 4 years post-transplant maintained an increase in the percent CD8+ T-cells in the bone marrow. $65 \%$ of the post-autologous transplant patients had clonal TCR gene rearrangements in the bone marrow, yet no patients had neutropenia or splenomegaly.

Conclusions: Though the LGL expansions were clonal and persistent in myeloma patients after autologous transplant, the lack of clinical sequelae suggests the clonal LGL expansion is a reactive immune response to autologous stem cell transplant. This immune response potentially has clinical benefits and should not be considered a neoplastic process.

\section{BMS015}

\section{Pancytopenia and "osteosis"}

\section{Martine Ffrench ${ }^{1}$}

\section{(1) Hospices Civils de Lyon, Lyon, France}

Background: Osteomyelosclerosis is classically found with metastatic carcinoma in bone marrow. However, while myelofibrosis (grade 1 to 3 ) is often observed in refractory cytopenias and / or acute leukemias, "osteosis" with thickened bone trabeculae is rarely reported in these diseases.

Patients and materials: We describe here three patients (two female and one male, 30, 50 and 57 years old respectively) presenting a pancytopenia,. Bone marrow aspirates were hypocellular and several core biopsies (1,2 and 3 respectively for the 3 patients) were performed showing mainly, either in the first or in the second biopsy, an enlargement of trabeculae with reticulin but not collagen fibrosis.

Results: For the youngest patient, an osteodensitometry was performed demonstrating an increased Z-score. For this patient, the biopsy measured only $1 \mathrm{~cm}$ : bone marrow was hypoplastic showing adipocytes, vessels and some lymphocytes and plasma cells without any myeloid cells. In the second case, bone marrow was hypercellular without any signs of myelodysplastic or myeloproliferative syndrome. In the third case, bone marrow was heterogeneous but globally hypoplastic with a dyserythropoiesis suggesting a myelodysplasia. In these 3 cases, CD34 expression analysis did not reveal excess of blasts when enlarged bone trabeculae were observed for the first time. Nevertheless, prognosis was poor: two patients, presented blast cells rapidly increasing and they died few months later (around 6 months). For the last patient, recently diagnosed, the outcome remains uncertain; however cytological and bone abnormalities appeared in less than three months.

Conclusions: Globally, these three cases raise the question whether a diagnosis of myelodysplastic syndrome unclassifiable may be proposed while cytological abnormalities appeared faint, when "osteosis" is observed in a context of pancytopenia.

\section{$\underline{\text { BMS016 }}$}

Core binding factor acute myeloid leukemia and c-kit mutations

Achille Pich ${ }^{1}$; Paola Francia di Celle ${ }^{2}$; Chiara Frairia ${ }^{3}$; Daniela Toppino $^{2}$; Ernesta Audisio ${ }^{3}$; Francesca Sismondi ${ }^{3}$; Stefano D'Ardia ${ }^{3}$; Cristiana Di Bello ${ }^{2}$; Emanuela Messa ${ }^{3}$; Filippo Marmont ${ }^{3}$; Umberto Vitolo ${ }^{3}$; Giorgio Inghirami ${ }^{4}$; Ludovica Riera ${ }^{5}$

(1) Department of Biomedical Sciences and Human Oncology, University of Turin, Italy (2) Center for Experimental Research and Medical Studies (CeRMS) (3) Department of Haematology, AOU S. Giovanni Battista Hospital, Turin, Italy. (4) 1Department of Biomedical Sciences and Human Oncology, Section of Pathology, University of Turin, It (5) Department of Biomedical Sciences and Human Oncology, University of Turin and Center for Experimenta

Background: $R U N X 1-R U N X 1 T 1[t(8: 21)]$ or $C B F B$ MYH11 [inv(16)] fusion transcript identifies the Core Binding Factor (CBF) Acute Myeloid Leukaemia (AML). CBF AML represents 5-8 \% of all AML and has a relatively favourable prognosis, with a good response to chemotherapy and longer complete remissions after treatment with high dose cytarabine in the consolidation phase. However, older age, CD56 expression and activating c-KIT mutations are reported to be associated with higher risk of relapse and shorter survival.

Aim of the study: To verify the incidence and prognostic value of c-KIT mutations in CBF AML.

Materials and methods: Bone marrow samples of 23 adult patients with de novo CBF AML, diagnosed at S. Giovanni Battista Hospital from 2000 to 2011, were retrospectively analysed. Fourteen patients $(60.8 \%)$ had inv(16) and $9(39.2 \%)$ had $t(8 ; 21)$. c-KIT mutations in exons $8,9,10,11,13,14$ and 17 were assessed by PCR amplification in combination with direct sequencing. All patients received standard induction chemotherapy with cytarabine, idarubicin and etoposide.

Results: 7/23 (30.4\%) patients showed c-KIT mutations. cKIT mutations were detected in 3/9 (33.3\%) patients with $t$ $(8 ; 21)$ and in 4/14 (28.6\%) patients with inv(16). M541L 
mutation (exon10) was found in 3 samples and D816V or $\mathrm{D} 816 \mathrm{H}$ or D816Y mutation (exon17) in 4. Two SNPs [K546K, I798I] were detected in 6 AML samples. FLT3ITD, FLT3D835 and NPM1 mutations rarely occurred. No difference in c-KIT mutation status was seen between cases with inv(16) or $t(8 ; 21)$ alone and cases with additional cytogenetic abnormalities. No association with sex, age, blood cell count, or peripheral blood and bone marrow blast cells at diagnosis was also found. On the contrary, LDH values were higher in mutated than non-mutated patients $(p=0.016)$. Complete remission and overall survival rates were similar in mutated and non-mutated patients.

Conclusions: Our results showed an overall incidence of cKIT mutation in $30.4 \%$ of cases, as previously reported in adult CBF AML patients. However, contrary to other studies, no prognostic value of c-KIT mutational status could be found, probably because of the relatively small number of patients.

\section{$\underline{\text { BMS017 }}$}

Systemic mastocytosis with clonal hematological nonmast cell lineage disease (SM-AHNMD) - a case report

Gorana Aralica ${ }^{1}$; Cedna Tomasovic-Loncaric ${ }^{1}$; Visnja Haris $^{2}$; Tajana Stoos-Veic ${ }^{3}$; Marija Skoro ${ }^{4}$; Rajko Kusec ${ }^{2}$

(1) Department of pathology University hospital Dubrava, Zagreb, Croatia (2) Department of Internal Medicine University Hospital Dubrava (3) Department of Cytology University Hospital Dubrava (4) Department of Cytology Univeristy Hospital Dubrava

Background: Mastocytosis is a rare clonal, neoplastic proliferation of mast cells that accumulate in one or more organ systems. There are several different types of mastocytosis. These diseases may occur at any age. Cuteaneous mastocytosis is usually presented at early age, while systemic mastocytosis is generally diagnosed after the second decade of life with a male to female ratio variation from 1:1 to 1:3.

Case report: Here, we present a case of a 46-year-old man who had a 5-year-old history of thrombocytosis and erosive gastritis and duodenitis. On admission, he complained of erytromelalgic pain in the foot. At presentation, he was in general good condition, with no palpable lymph nodes or spleen. On gastroscopy, there were multiple antral erosions. On abdominal US, thickened gall bladder wall was noticed. Blood tests were abnormal $(E 5.18 \times 1012 / \mathrm{L}$, hemoglobin $158 \mathrm{~g} / \mathrm{L}, \mathrm{L} 17.7 \times 106 / \mathrm{L}$ [neutrophil granulocytes $3.40 \times$ 106/L, 68.7\%], Trc $\left.997 \times 10^{9} / \mathrm{L}\right)$. Bone marrow aspirate was normocellular with normoblastic erythropoiesis and mature granulopoiesis. Megakaryocytes were numerous, large, with hyperlobulated nuclei consistent with diagnosis of MPN-ET. Spindle shaped mast cells were found only after reviewing of bone marrow aspirates. Bone marrow biopsy showed slightly hypercelluar (65\%) bone marrow with compact, predominantly peritrabecular infiltrates $(20 \%$ of cellularity) of spindle-shaped and round mast cells with CD117 and CD25 positive immunohistochemical reaction. Also, there were increased numbers of large, hyperlobulated megakaryocytes, focally in small groups. Myelopoiesis and erythropoiesis were increased in number, with maturation. Molecular tests confirmed D816V mutation in c-kit and JAK-2 V617F mutation.

Conclusion: Our diagnosis is systemic mastocytosis with clonal hematological non-mast cell lineage disease (SMAHNMD), essential thrombocytemia (MPN-ET).

\section{BMS018}

Immunofluorescence staining with an antipromyelocytic leukemia antibody establishes the diagnosis of acute promyelocytic leukemia in patients with PMLRARA fusion gene undetectable by fluorescence in situ hybridization

Sergej Konoplev ${ }^{1}$; Khaled Alayed ${ }^{1}$; C. Cameron Yin ${ }^{1}$; Nikolay D. Dimov ${ }^{1}$; L. Jeffrey Medeiros ${ }^{1}$; Carlos E. Bueso-Ramos ${ }^{1}$

(1) The UT MD Anderson Cancer Center, Houston, TX, USA

Background: Patients with APL are at risk of lethal coagulopathy if therapy is delayed. APL is characterized by $t$ (15;17)(q22;q21)/PML-RARA, and many institutions rely on FISH and/or RT-PCR for rapid detection of PML-RARA. In rare patients, however, PML-RARA is undetectable by FISH and is only detected by RT-PCR. Immunofluorescence staining with anti-promyelocytic leukemia (PML) antibody is a rapid method that can be performed on blood or bone marrow aspirate smears in real time; a finely granular pattern of staining highly correlates with PML-RARA.

Materials and methods: We searched our files for APL cases with positive immunofluorescence staining using antiPML antibody (courtesy of K. Chang, MD Anderson) that were also positive by RT-PCR, but negative by FISH. The anti-PML antibody has been was reported (Blood 85: 3646, 1995). I

Results: We identified 3 patients, all women, 33 to 57 years of age. In each patient conventional cytogenetics was diploid and RT-PCR was positive for PML-RARA. FISH with a LSI PML/RARA dual color, dual fusion probe (Vysis, Inc) was negative in all patients. In 1 patient, the LSI RARA dual color, breakapart probe (Vysis) also did not detect RARA rearrangement. In this patient, we performed an oligonucleotide-based array CGH analysis using a $315 \mathrm{~K}$ 
oligonucleotide array (OncoChipTM AML/MDS panel 1, Signature Genomic Laboratories) and detected $t(15 ; 17)(\mathrm{q} 22$; q21) with PML breakpoint at 15q24.1(72,103,072$72,103,231)$ within the breakpoint cluster in intron 3 and RARA breakpoint at $17 \mathrm{q} 21.2(35,743,768-35,743,888)$ within intron 2 . All patients were treated with ATRA, arsenic trioxide, and chemotherapy, achieved complete remission and were alive and well 2-14 years after diagnosis.

Conclusions: PML immunofluorescence staining allows rapid confirmation of the diagnosis of APL and is helpful in rare patients in whom the PML-RARA fusion gene undetectable by FISH, facilitating rapid initiation of appropriate therapy.

\section{BMS019}

\section{T-cell large granular lymphocyte proliferations analysis -} report on 55 cases

Monika Prochorec-Sobieszek ${ }^{1}$; Grzegorz Rymkiewicz ${ }^{2}$; Hanna Makuch-Lasica ${ }^{1}$; Anna Szumera-Ciezkiewicz ${ }^{1}$; Katarzyna Michalak ${ }^{1}$; Olga Szymanska-Giemza ${ }^{1}$; Renata Maryniak $^{1}$; Krzysztof Warzocha ${ }^{1}$

(1) Institute of Hematology and Transfusion Medicine, Warsaw, Poland (2) The Maria Sklodowska-Curie Memorial Cancer Center and Institute of Oncology

Background: T-cell large granular lymphocyte (T-LGL) proliferations represent a spectrum of lymphoproliferative disorders that either may occur as reactive, polyclonal LGL expansion, chronic oligo/monoclonal asymptomatic LGL lymphocytosis or may fulfill clinical criteria of T-LGL leukemia.

Aim of the study: The clinical and biological characteristics of patients with T-LGL proliferations were studied.

Materials and methods: A total 55 patients with symptomatic T-LGL lymphocytosis were selected. The results of clinical, histopathological, immunohistochemical, flow cytometric and molecular of blood or bone marrow examinations (TCR and IG genes rearrangements) were analyzed. Results: T-LGL leukemia was recognized in 43 cases; 12 patients had reactive T-LGL lymphocytosis. In T-LGL leukemia group neutropenia was observed in $93 \%$ of cases; splenomegaly and autoimmune were identified in $43 \%$ and $50 \%$ respectively. Clinical signs and variations of laboratory tests symptomatic reactive T-LGL lymphocytosis were similar to T-LGL leukemia except for lower T-LGL lymphocytosis and less frequent neutropenia. Bone marrow trephines from T-LGL leukemia patients showed interstitial clusters and intrasinusoidal linear infiltrations of CD3+/CD8 +/CD57+/granzymeB+ lymphocytes, reactive lymphoid nodules and decreased or normal granulocyte precursors count with left-shifted maturation. In three-color flow cytometry (FCM) T-LGL leukemia cells demonstrated $\mathrm{CD} 2, \mathrm{CD} 3$ and $\mathrm{CD} 8$ expression as well as a combination of CD16, CD56 or CD57. Abnormalities of other T-cell antigens expression (especially CD5, CD7, CD43, CD2, and CD3) were also detected. In the polyclonal T-LGL lymphocytosis T-cells were dispersed in the bone marrow and the expression of pan-T cell antigens in FCM was normal. Simultaneous rearrangements of TCRB and TCRG were detected in $70 \%$ of T-LGL leukemia; the $\mathrm{V} \gamma$ If, $\mathrm{V} \gamma 10$ $\mathrm{J} \gamma$ region was most frequently rearranged and additional IGK/ IGL rearrangements without B-cell proliferation were observed in $18 \%$.

Conclusions: Symptomatic patients with T-LGL lymphocytosis represented a spectrum of T-LGL proliferations though monoclonal expansions were most frequently observed. The histopathological and cytometric studies revealed certain disease-specific features however TCR genes rearrangements need to be confirmed.

\section{BMS020}

LMO2 immunostaining on marrow core biopsies in benign and malignant hematolymphoid conditions

Kai Zhang $^{1}$

(1) Geisinger Health System, USA

Background: LMO2 immunostaining has been used in the differential diagnoses of small B tumor in nodal and extranodal sites. LMO2 immunostaining pattern in bone marrow (BM) core bx is not well studied.

Aim of the study: To characterize LMO2 immunostaing pattern and /or cell types in BM core bx in various benign and malignant hematolymphoid conditions.

Materials and methods: Materials and methods: We investigated immunostaining of LMO2 on conventional and tissue microassay sections in 340 cases of BM core biopsies in following 3 groups of cases. 1). 20 cases of benign marrow cases included hemolytic anemia, lymphoma staging and aplastic anemia. 2). 150 cases of stem cell-myeloid tumors included 20 cases of MDS (RA, $n=5$, RARS, $n=5$, RAEB1, $n=5$ and RAEB2, $n=5), 20$ cases of MPN, 7 cases of AML with 2 cases of M6. 3). 200 lymphoid tumors included ALL, $n=42$, FL, $n=24$, B-CLL, $n=65$, MCL, $n=5$, HCL, $n=20$, and myeloma, $n=64$.

Results: Benign and dysplastic nucleated red blood cells (nRBCs) and megakaryocytes (megs) of group 1 and 2 cases demonstrated a strong nuclear staining of LMO2. All myeloid cells were negative for LMO2 in group 1 cases. Some of small clusters of immature granulocytes or blasts in groups 2 cases showed moderate positivity. 7 cases of 
AML were all positive for LMO2 with weak to strong intensity; 2 of these 7 cases being acute erythroid leukemia with leukemic erythroblasts exhibiting much stronger nuclear positivity compared to leukemic blasts of other AML cases that tended to have weak and moderate positivity. 40 out of $42(95 \%)$ ALL cases were positive for LMO2 with most cases (36 of 42) showing moderate or strong positivity. One out of 24 (4.1\%) cases of FL was positive for LMO2 with moderate intensity. None of the cases of B-CLL, MCL, HCL and myeloma were positive for LMO2.

Conclusions: 1. LMO2 is great marker for both benign and neoplastic nRBCs and megakaryocytes. 2. Only small percentage of FL involving BM showing positive for LMO2. 3 . Caution should be taken when interpretation of LMO2 staining in evaluating small B cell tumor in BM core bx since many cell types can be positive for LMO2.

\section{$\underline{\text { BMS021 }}$}

\section{A morphometric study of bone marrow angiogenesis in Waldenström Macroglobulinemia with clinicopathological correlations}

T Tzenou ${ }^{1}$; G. Levidou ${ }^{2}$; N. Kavantzas ${ }^{3}$; K. Xirokosta ${ }^{1}$; D. Maltezas $^{1}$; P. Korkolopoulou ${ }^{3}$; E. Koulieris ${ }^{1}$; C. Kalpadakis ${ }^{4}$; TP. Vassilakopoulos ${ }^{4} ;$ MK. Angelopoulou ${ }^{4} ;$ P. Panayiotidis ${ }^{4}$; GA. Pangalis ${ }^{4}$; E. Patsouris ${ }^{3}$; MC. Kyrtsonis ${ }^{1}$

(1) 1st Dept of Propaedeutic Internal Medicine, University of Athens, Medical School Athens, Greece (2) Department of Pathology, Kapodistrian University of Athens, Medical School, 11527 Athens, Greece (3) Department of Pathology, University of Athens, Medical School, Laiko Hospital, Greece (4) Department of Hematology and Bone Marrow Transplantation, University of Athens, Medical School, Lai

Background: Bone marrow angiogenesis has recently been implicated in the pathophysiology and course of various haematological malignancies. Little is known, however, about the significance of this phenomenon Waldenstrom's Macroglobulinemia (WV).

Materials and methods: In this study, we evaluated various morphometric characteristics of microvessels, highlighted by means of anti-CD34 immunohistochemistry, in the bone marrow of 36 patients with typical WM. Microvessel density (MVD) and several size- and shape-related parameters were quantified in the region of most intense vascularization using image analysis. Two main parameters were evaluated, microvessel density (MVD) and total vascular area (TVA) and were correlated with clinicopathological characteristics, time to first treatment and patients' overall survival.

Results: MVD ranged from 7 to 393 (median value 32), whereas TVA ranged from 2681 to $69160 \mu \mathrm{m} 2$ (median value 27762). Both TVA and MVD were not correlated with clinical and laboratory disease parameters. Symptomatic patients $(84 \%)$ received treatment while the others $(16 \%)$ were regularly followed. Median time to first treatment (TFT) was 2 months while median overall survival was 89 months. Patients' overall survival was adversely affected by increased TVA $(p=0.03)$. Moreover, the presence of increased TVA was correlated with longer median time to first treatment (TFT) $(p=0.03)$. However, MVD failed to provide any information in this regard.

Conclusions: Our data suggests that the generation of bone marrow microvessels indicate an increased risk of in WM, whereas Increased TVA may prove a powerful predictor of TFT and OS. Further studies in larger cohorts are warranted to validate and extend our results.

\section{BMS022}

Large Granular T-cell Lymphocyte Leukemia (T-LGL leukemia): A case report with atypical features

Braulio Martínez-Benítez ${ }^{1}$; Morelos Villaseñor ${ }^{1}$; Ana Lilia Morales-Leyte $^{1}$; Paris Ramos ${ }^{1}$; Daniel Rosales-López ${ }^{1}$; David Valdés ${ }^{1}$; Ricardo Aguilar ${ }^{1}$; Yvette Neme-Yunes ${ }^{1}$; Carmen Lome-Maldonado ${ }^{1}$

\section{(1) INCMNSZ Mexico City, Mexico}

Background: T-LGL leukemia is a rare entity that presents more commonly in adults between 45 and 75 years, being a rare condition $(<3 \%)$ before the age of 25 . It is described as a heterogeneous disorder characterized by a persistent and significant peripheral blood increase ( $>6$ months) of large granular lymphocytes $(2-20 \times 10 / \mathrm{L})$. It has been associated with an immune system deregulation and has an indolent clinical course, although two thirds of patients present with symptoms comprising hematologic (cytopenias) and rheumatologic alterations. The large granular lymphocyte proliferation affects peripheral blood, bone marrow, liver and spleen. The morphology is that of a T-lymphocyte with mature chromatin and azurophilic cytoplasmic granules. They are $\mathrm{CD} 3+, \mathrm{CD} 8+$ and express cytotoxic cytoplasmic granules by immunohistochemistry. It is important to note that there is no consensus regarding the number of lymphocytes needed in order to make a diagnosis of T-LGL leukemia. Therefore, cases with $<2 \times 10$ that meet the other proposed criteria are still considered as T-LGL leukemia.

Aim of the study: To present a T-LGL leukemia case report with clinical atypical features.

Materials and methods: In a period of 9 years (20032012), five cases of T-LGL leukemia were identified, four of which were adults over 50 years that presented with an indolent clinical course and with the classical clinical 
features described for the disease. The other case was that of a 21 year-old female patient in which the clinical data was reviewed and immunohistochemistry was performed in the bone marrow biopsy. The primary antibodies found were: CD20, CD3, CD4, CD8, CD2, CD7, CD5, CD56, CD57, TIA-1, granzyme and perforin.

Results: This is the case of a 21 year-old woman that presented with a clinical course consisting of B symptoms, epistaxis, occipital headache and blurred vision 8 months prior to the diagnosis. She also had thrombocytopenia and splenomegaly as well as an increase in antinuclear (1:160) and anti double stranded DNA antibodies titer. In the bone marrow aspirate the presence of large granular lymphocytes was confirmed.

In the bone marrow aspirate the presence of large granular lymphocytes was confirmed. The bone marrow biopsy showed an interstitial lymphoid infiltrate comprising a population of mature lymphocytes that were $\mathrm{CD} 3+, \mathrm{CD} 8+$, Tia1+, $\mathrm{CD} 2+, \mathrm{CD} 7+, \mathrm{CD} 4+, \mathrm{CD} 56-, \mathrm{CD} 57+$ on flow cytometry, consistent with the diagnosis of T-LGL leukemia.

Conclusions: This case shows the heterogeneity and diversity that can occasionally be seen in T-LGL leukemia. Despite the peculiar clinical characteristics (young age of the patient and lack of a classic leukemic phase) this case presented with the typical morphological and immunohistochemical features required for the diagnosis of this entity.

\section{BMS023}

\section{Bone marrow findings in angioimmunoblastic T-cell lymphoma with modern immunophenotyping}

Sarah Ondrejka ${ }^{1}$; Eric D. Hsi ${ }^{1}$; Craig Portell ${ }^{1}$; Jonathan Said $^{2}$

(1) Cleveland Clinic, Cleveland, OH, USA (2) UCLA, Los Angeles, CA, USA

Background: Angioimmunoblastic T-cell lymphoma (AITL) is a peripheral T-cell lymphoma of putative follicular helper (TFH) origin that presents commonly with high stage disease and frequent bone marrow involvement. Bone marrow (BM) manifestations can range from paraneoplastic changes to diffuse involvement.

Aim of the study: To examine BM biopsies from patients with AITL for the spectrum of morphologic and immunophenotypic features and to correlate histologic features with event free survival (EFS) and overall survival (OS).

Materials and methods: Available bone marrow biopsies from 17 patients with AITL were studied for histopathologic features including overall cellularity, \% marrow involvement, cellular composition, presence of large lymphoma cells and expression of TFH markers (CD10, PD1, CXCL13 and ICOS) by immunohistochemistry (IHC).

Results: Staging BM biopsies were involved by lymphoma in 10 of 17 AITL patients (58 \%) at diagnosis. The median percent BM involvement was $20 \%$ (range 10-50\%) and the pattern of involvement varied with non-paratrabecular aggregates in $5 / 10$, exclusively paratrabecular infiltrates in $0 / 10$, diffuse/interstitial infiltrate in $2 / 10$, and both intertrabecular and paratrabecular infiltrates simultaneously in $3 / 10$. The lymphoma composition had either a mixed inflammatory appearance ( 5 cases) or a more easily recognizable monomorphic appearance composed of aggregates of atypical small to medium sized lymphocytes with clear cytoplasm (5 cases). Scattered, large transformed cells were 0 /high-powered $400 \times$ field (HPF) in 6 patients, $1-3 / \mathrm{HPF}$ in 3 patients, and $\geq 10 / \mathrm{HPF}$ in one patient. Kaplan Meier (KM) analysis with log rank testing showed no significant differences in EFS or OS based on marrow involvement (positive vs negative). However, in patients with BM involvement, presence of $\geq 1$ large cell/HPF had a significantly shorter EFS than patients without large cells (median EFS of 1.5 vs. 53.5 months, $p=0.005$ [log rank test]). The effect of $\geq 1$ large cell/HPF showed a trend toward decreased OS but did not reach statistical significance. There was no difference in EFS or OS according to the tumor composition. IHC for TFH-phenotype showed individual concordance of $100 \%$ (CD10), $83 \%$ (PD1), $83 \%$ (CXCL13) and $75 \%$ (ICOS) in marking the neoplastic cells when the corresponding nodal lymphoma was positive for that marker. Conclusions: AITL frequently involves the BM at diagnosis, with a variable histologic appearance that may consist of polymorphous inflammatory-like or monomorphous nodular aggregates of atypical lymphocytes. In AITL patients with BM involvement at diagnosis, EFS can be predicted by the presence of any large cells. TFH markers tend to remain concordant between BM and lymph node. Use of multiple TFH markers is advised in order to demonstrate TFH lineage in the BM.

\section{BMS0024}

A genetically engineered mouse model for studies of the hematopoietic system using fluorescence technology

Cynthia S Snyder ${ }^{1}$; Robert M. Hoffman ${ }^{1}$; Michael Bouvet ${ }^{1}$

\section{(1) University of California, San Diego, CA, USA}

Background: The mouse is the premier animal model for studying vertebrate development and disease and serves as the mammalian model of choice for testing of novel therapeutics. Recent interest in using fluorescence technology for studies of living cells has surged, reflecting increased availability of genetically-encoded fluorescent proteins for multispectral imaging, advances in fluorescence technology, and 
improved methodologies for dynamic in vitro and in vivo studies of living cells and tissues. We sought to develop a mouse model for multi-spectral, fluorescence-based studies of hematopoiesis and hematopoietic disease.

Methods: To develop this model we used Cre/loxP technology. $\mathrm{mT} / \mathrm{mG}$ Cre-reporter mice constitutively express tdTomato, a red fluorescent protein. Following exposure to Cre recombinase, the conditional $\mathrm{mT} / \mathrm{mG}$ transgene undergoes intramolecular recombination, resulting in expression of green fluorescent protein (GFP). Both tdTomato and GFP are membrane-targeted, yielding bright, membranelocalized tdTomato $(\mathrm{mT})$ or GFP (mG) fluorescence useful for imaging and fluorescence activated cell sorting (FACS) studies. For studying the hematopoietic system, we used Tie2-Cre transgenic mice. TIE2/TEK, a receptor tyrosine kinase, is expressed on hematopoietic and endothelial cells. Tie2-Cre and $\mathrm{mT} / \mathrm{mG}$ mice were crossed to generate Tie2Cre; $\mathrm{mT} / \mathrm{mG}$ double transgenic mice. To characterize this model for studying the hematopoietic system, we performed bright light and fluorescence imaging, FACS analysis, and confocal microscopy of hematopoietic tissues from Tie2$\mathrm{Cre} ; \mathrm{mT} / \mathrm{mG}$ experimental mice and controls.

Results: Tie2-Cre;mT/mG mice demonstrated bright GFP expression in hematopoietic tissues, including fetal liver, bone marrow, and spleen. Lymph nodes, other lymphoid tissues including Peyers patches, and vascular endothelium also expressed GFP. Surrounding tissues expressed tdTomato. The membrane-localization of $\mathrm{mG}$ and $\mathrm{mT}$ fluorescence enabled distinction of single cells in confocal images of Tie2-Cre;mT/ $\mathrm{mG}$ hematopoietic tissues and facilitated FACS analysis.

Conclusions: This fluorescent mouse model enables multispectral analysis of a variety of hematopoietic tissues and should prove valuable for studies of hematopoiesis and hematopoietic malignancy entailing fluorescence technology.

\section{Lymphoma Symposium}

\section{LYS001}

Revising the historical collection of epithelioid cell-rich lymphomas of the Kiel Lymph Node Registry: what is Lennert's lymphoma nowadays?

Sylvia Hartmann ${ }^{1}$; Claudio Agostinelli ${ }^{2}$; Wolfram Klapper ${ }^{3}$; Penelope Korkolopoulou ${ }^{4}$; Karoline $\mathrm{Koch}^{5}$; Teresa Marafioti ${ }^{6}$; Pier Paolo Piccaluga ${ }^{7}$; Efstratios Patsouris ${ }^{4}$; Stefano Pileri ${ }^{2}$; Martin-Leo Hansmann ${ }^{8}$

(1) Senckenberg Institute of Pathology, Goethe University, Frankfurt am Main, Germany (2) Department of Haematology and Oncological Sciences 'L. and A. Sera'gnoli', Haematopathology Section, (3) Institute of Pathology, Hematopathology Section and Lymph Node Registry, University Hospital
Schles (4) Department of Pathology, University of Athens, Medical School, Greece (5) 2Institute of Pathology, Haematopathology Section and Lymph Node Registry, Universita"tsklinikum Sch (6) University College London (7) Haemolymphopathology - S. Orsola-Malpighi Hospital University of Bologna (8) Institute of Pathology, University Hospital Frankfurt

Background: Lennert's lymphoma is a rare variant of peripheral T-cell lymphoma (PTCL) not otherwise specified (NOS).

Aim of the study: The aim of this study was to further characterize this tumour.

Materials and methods: Historical material of 97 lymphomas with a high content of epithelioid cells, collected at the Kiel Lymph Node Registry were reviewed, by applying immunohistochemistry and current diagnostic criteria.

Results: Among all cases revised, various B-cell lymphoma entities (25 cases), Hodgkin lymphomas (21 cases) and PTCL subtypes (48 cases) could be identified. A distinctive subgroup of eight PTCLs was found that were regarded as genuine Lennert's lymphomas. These cases were characterized by mild atypia, a non-activated cytotoxic phenotype TIA1positive+ and granzyme B-negative, and a substantial lack of follicular T-helper (TFH) cell markers. Among the other PTCLs, including angioimmunoblastic T-cell lymphoma and PTCL NOS, many cases with positivity for more than three TFH cell associated molecules were recorded.

Conclusions: Our study shows that, according to current criteria, Lennert's lymphoma is a rare but distinctive entity among epithelioid cell-rich lymphomas, differing on grounds of morphology and immunophenotype from other PTCL subtypes. An additional finding is the broad morphological spectrum of epithelioid-cell rich PTCLs showing a TFH cell phenotype.

\section{LYS002}

Diagnostic accuracy of peripheral T-cell lymphoma using a defined immunophenotypic and molecular genetic approach from the North American PTCL Study Group

Eric D. Hsi ${ }^{1}$; Jonathan $\mathrm{Said}^{2}$; William $\mathrm{Macon}^{3}$; Scott Rodig $^{4}$; Randy D. Gascoyne ${ }^{5}$; Sarah Ondrejka ${ }^{1}$; David Dorfman $^{4}$; Matthew Maurer ${ }^{3}$; Ahmet Dogan ${ }^{3}$

(1) Cleveland Clinic, Cleveland, OH USA (2) UCLA (3) Mayo Clinic (4) Brigham and Women's Hospital (5) British Columbia Cancer Agency

Background: The diagnosis of peripheral T-cell lymphoma (PTCL) is difficult due to the rarity and pathologic heterogeneity of this group of lymphomas. Accurate diagnosis and subclassification relies on correlating histologic, 
immunophenotypic, molecular genetic, and clinical data. However, evidence-based guidelines on the appropriate diagnostic work-up of PTCL are lacking.

Aim of the study: The objective of this study was to evaluate the diagnostic accuracy of a tiered approach to immunohistochemical and molecular genetic characterization in a large series of PTCLs by a panel of experienced hematopathologists.

Materials and methods: 7 hematopathologists from 5 academic institutions gathered 374 cases for review. 6 cases of cutaneous T-cell lymphoma (CTCL) were excluded after final review in addition to 29 non-PTCL cases submitted as control cases to mimic diagnostic practice during the review. Each case received sequential independent tier 0, 1 and 2 diagnoses by 3 pathologists, based on review of hematoxylin and eosin (HE) stained slides accompanied by basic demographic data (tier 0 ), then by evaluation of tier 1 (CD3, CD5, CD10, CD20, CD21, CD30, CD45, PAX5) immunohistochemistry (IHC), and subsequently by examination of tier 2 IHC (CD2, CD4, CD7, CD8, CD23, PD1, CD56, EBER, ALK, TIA1, TCRd, TCRbF1), respectively. A tier $2 \mathrm{~b}$ diagnosis was then made after T-cell receptor and/ or immunoglobulin gene rearrangement data were available. A final consensus diagnosis (unanimous agreement required for consensus) was rendered after full discussion of each case by the 3 reviewers, after all available clinical data were provided.

Results: 4,032 individual diagnoses leading to 339 final consensus PTCL diagnoses were rendered. 341 (91\%) cases had complete phenotypic data and 241 had gene rearrangement data. There was no bias toward a particular PTCL subtype due to missing data. In PTCL cases, only $16.3 \%$ of tier 0 individual reviewer diagnoses were specific as to WHO PTCL subtype. Tier 1 diagnoses resulted in increased specific WHO diagnoses to $36.9 \%$. After Tiers 2 and $2 \mathrm{~b}$, reviewers were able to classify cases according to specific WHO subtypes in $82.7 \%$ and $85.9 \%$ of the time, respectively, demonstrating a significant increase in diagnostic certainty after a complete IHC panel. Gene rearrangement only contributed to a change in diagnosis in 51 out of 650 $(8 \%)$ individual reviews. The percentage of atypical infiltrate or reactive diagnoses was $20.5 \%$ after tier 0 (H\&E) and was reduced significantly as additional information was added $(9.3 \%, 3.8 \%$, and $1.3 \%)$ in tiers 1,2 , and $2 b$ respectively. Across all 374 cases, a small number of cases ( $n=28,7.5 \%$ ) showed no agreement among the 3 independent reviewers after tier $2 \mathrm{~b}$ and required debate. However, these generally represented refinement of the subclassification of a T-cell lymphoma, rather than decisions between reactive and PTCL. The most common disagreements were between PTCL, not otherwise specified (NOS) vs unclassifiable T-cell lymphoma, and PTCL, NOS vs angioimmunoblastic T-cell lymphoma, respectively.
Conclusions: We demonstrate the diagnostic accuracy among experienced hematopathologists using a defined IHC panel in which a consensus diagnosis was achieved in $93 \%$ of PTCLs. A tiered approach to IHC is recommended when a PTCL is under differential diagnostic consideration since the first tier can greatly reduce the main question of whether a lesion is reactive or a type of lymphoma. This can lead to more efficient use of the expanded tier 2 panel that will enable diagnosis and specific subclassification of PTCL. Knowledge of specific clinical information is most important when there is a history of HTLV1 infection or prior CTCL occurring in a non-cutaneous site. Gene rearrangement studies are not required in the great majority of cases once the recommended IHC panel is performed, but can be helpful in a small number of cases. This evidence-based approach to the diagnosis of PTCL should be considered by practicing pathologists, for clinical trial design, and by policy makers regarding required ancillary studies in this group of diseases.

\section{LYS003}

\section{Molecular diagnosis of nodal peripheral T-cell lymphomas; results of a phase 3 diagnostic accuracy study}

Pier Paolo Piccaluga ${ }^{1}$; Antonio De Leo ${ }^{2}$; Maura Rossi ${ }^{3}$; Maria Antonella Laginestra ${ }^{2}$; Francesco Bacci $^{2}$; Elena Sabattini ${ }^{2}$; Anna Gazzola ${ }^{2}$; Claudia Mannu ${ }^{2}$; Maria Rosaria Sapienza ${ }^{3}$; Claudio Agostinelli ${ }^{2}$; Fabio Fuligni ${ }^{3}$; Clara Bertuzzi ${ }^{2}$; Sylvia Hartmann $^{4}$; Martin L. Hansmann ${ }^{4}$; Roberto Piva ${ }^{5}$; Javeed Iqbal $^{6}$; John C. Chan ${ }^{6}$; Giorgio Inghirami ${ }^{7}$; Stefano A. Pileri ${ }^{2}$

(1) Haemolymphopathology - S. Orsola-Malpighi Hospital University of Bologna (2) Hematopathology Section, Department of Hematology and Oncology "L. and A. Seràgnoli", S. Orsola-Malp (3) Haemolymphopathology Unit - S. Orsola-Malpighi Hospital - University of Bologna Italy (4) Senckenberg Institute of Pathology, Goethe University, Frankfurt am Main, Germany (5) Department of Pathology and Centre for Experimental Research and Medical Studies (CeRMS), University (6) Departments of Pathology and Microbiology, University of Nebraska Medical Center, Omaha, NE (7) 1Department of Biomedical Sciences and Human Oncology, Section of Pathology, University of Turin, Italy

Background: The differential diagnosis among the commonest peripheral T-cell lymphomas (PTCLs) (PTCL not otherwise specified, NOS; angioimmunoblastic T-cell lymphoma, AITL; and anaplastic large cell lymphoma, ALCL) is difficult, the morphologic and phenotypic features being largely overlapping.

Aim of the study: We performed a phase 3 diagnostic accuracy study to test the ability of gene expression profiles (GEP, index test) to identify PTCL subtypes. 
Materials and methods: We studied 244 PTCLs, including 158 PTCLs/NOS, 63 AITLs, and 23 ALCLs ALK-, divided into training $(N=41)$, test $(N=71)$ and validation $(N=132)$ sets. GEPs were generated by either Illumina Whole Genome DASL Assay $(N=112)$, or Affymetrix HG-U133 2.0 plus $(N=132)$. The classification method was based on a support vector machine algorithm, respecting the STARD QUADAS and REMARK guidelines. The reference standard was an expert pathological diagnosis according to the WHO classification.

Results: First, we identified molecular signatures discriminating either AITL and ALCL ALK- from PTCL/NOS in the training set. Secondly, we tested the diagnostic accuracy of the index tests. The overall accuracy of the tests was remarkable, being $76-95 \%$ for the AITL classifier and 85-98 \% for the ALCL ALK- one, in the test and validation set of cases, respectively. Of interest, border line cases (i.e. CD30+ PTCLs/NOS) were correctly classified with high efficiency. Noteworthy, in all the conditions, the post-test probability definitely improved the pre-test probability of the target condition, outlining the practical relevance of the tool for diagnostic purposes.

Conclusion: Our findings support the usage of a molecular classifier as additional tool in the diagnostic work up of nodal PTCLs.

\section{LYS004}

\section{Targeting the NF- $\kappa B$ pathway in peripheral T-cell lymphomas}

Miguel A Piris ${ }^{1}$; Lina Odqvist ${ }^{2}$; Santiago Montes-Moreno ${ }^{1}$; Esperanza Martin ${ }^{2}$

(1)Pathology Department, Hospital Marques de Valdecilla, Santander, Spain, (2) Spanish National Cancer Research Center (CNIO), Molecular Pathology Programme

Background: Peripheral $\mathrm{T}$ cell lymphomas are aggressive malignancies, which today lack any targeted therapies. Deregulated nuclear factor kappa B (NF-KB) activity has been associated with a number of lymphoproliferative diseases, including peripheral $\mathrm{T}$ cell lymphomas (PTCLs). Even though targeting the NF-KB pathway has been suggested as a therapeutic strategy in lymphomas, the role of NF- $\mathrm{KB}$ signaling in lymphomagenesis is still poorly understood, and further studies are needed in order to develop effective and safe NF-KB inhibitors.

Aim of the study: Is NF- $\mathrm{KB}$ a rational target in peripheral Tcell lymphomas?

Results: In this study, we analyzed the nuclear expression of NF- $\mathrm{KB}$ by immunohistochemistry in a series of 77 primary PTCL samples and observed that both the classical and alternative NF- $\mathrm{kB}$ pathways were activated in a subset of cases associated with a significantly worse overall survival. We subsequently observed that the NF-KB-inducing kinase (NIK) was overexpressed in a subset of PTCL cell lines and primary samples and that genetic inhibition of NIK was able to reduce both classical and alternative NF- $\mathrm{KB}$ activity. Gene expression microarray analysis after NIK knockdown revealed a decrease in the expression of NF- $\mathrm{kB}$ target genes and modulated expression of several key genes involved in tumor growth and survival. Notably, NIK knockdown led to a dramatic induction of apoptosis in NIK-overexpressing T cell lymphoma cell lines, whereas cell lines with low NIK levels were unaffected. Furthermore, NIK knockdown produced a more potent cytotoxic effect than $\operatorname{did} I K K \alpha$ or IKK $\beta$ Knock-down in these cells.

Conclusions: The results of this study suggest that NIK is a promising molecular target in NIK-overexpressing PTCL, a conclusion that should be taken into account for further validation and development of specific NIK inhibitors.

\section{LYS005}

\section{Angioimmunoblastic T-cell lymphoma (AITL) is the most} prevalent T-cell lymphoma entity in Western Europe

Marie Parrens, Antoine Martin, Laurence Lamant, Richard Delarue, Francoise Berger, Flavie Abion, Céline Bossard, Brigitte Bouchindhomme, Josette Briere, Danielle Canioni, Frédéric Charlotte, Peggy Dartigues, Pierre Dechelotte, Jean-François Emile, Bettia Fabiani, Corinne Haioun, Albane Ledoux-Pilon, Laurent Martin, Karine Montagne, François Michiels, Thierry Molina, Martine Patey, Patrick Tas, Michel Peoch, Barbara Petit, Tony Petrella, JeanMichel Picquenot, Thérèse Rousset, Isabelle Quintin-Roue, Marie-Christine Rousselet, Dimitri Salameire, Isabelle Soubeyran, Sylvie Thiebault, Olivier Tournillac, Luc Xerri, Virginie Fataccioli, Georges Delsol, Laurence de Leval, Philippe Gaulard for the Lymphopath Network and the Tcell lymphoma Tenomic Consortium

Département de Pathologie, Hôpital Pessac, CHU Bordeaux; Département de Pathologie \& INSERM, U955, Hôpipal Henri Mondor, Créteil, France; Institut de Pathologie. CHU Vaudois, Lausanne. Switzerland.

Background: Lymphoid malignancies derived from $\mathrm{T}$ and NK-cells (PTCLs) constitute a heterogeneous group of uncommon disease entities, with marked geographic variation in their distribution among different parts of the world. The most recent data from the International T-cell Lymphoma Project based on a retrospective analysis of PTCLs diagnosed between 1990 and 2002 indicate that PTCL, not otherwise specified (PTCL, NOS) represents the most 
common PTCL worldwide (25,9 \%), followed by angioimmunoblastic T-cell lymphoma (AITL) $(18,5 \%)$. Over the last few years, a better characterization of the cellular origin and pathophysiology of PTCLs has led to the development of new diagnostic markers.

Aim of the study: To characterize the epidemiology of mature T/NK-cell malignancies in Western Europe (France and bordering countries) and to examine whether the availability of new tools/antibodies and changing concepts over the past years might have induced a change in the diagnoses habits.

Materials and methods: The histopathological diagnosis of PTCL entities according to the 2008 WHO classification were collected through two independent sets of PTCLs in France and bordering countries.

Results: Over the past 2 years (2010-2011), 933 newly diagnosed non-cutaneous PTCLs were reviewed in reference centers through the prospective network "Lymphopath" aiming to review any newly diagnosed lymphoma in France. According to the 2008 WHO classification, the 933 PTCLs comprised: AITL $(n=314,33,6 \%)$, PTCL, NOS $(n=239,25,6 \%)$, ALK-positive anaplastic large cell lymphoma (ALCL) $(n=78,8,3 \%)$, ALK-negative ALCL ( $n=$ 72, 7,7\%), extranodal NK/T-cell lymphomas (ENKTL, $n=$ $59,6 \%)$, enteropathy-associated T-cell lymphoma $(n=33$, $4 \%)$, hepatosplenic T-cell lymphoma $(n=7,1 \%)$, Adult T leukemia/lymphoma (HTLV1+, $n=32,3 \%$ ), others entities or unclassifiable PTCLs $(n=99,11 \%)$.

A high prevalence of AITL was also found in an independent set of PTCLs retrospectively collected in the framework of a multicentric T-cell lymphoma research consortium "Tenomic" where non-cutaneous PTCL with frozen material available $(n=623)$ from 1999 to 2009 in France and Belgium were retrieved and collegially reviewed for consensus diagnosis. In this collection, AITL $(n=288,46 \%)$ also outnumbered PTCL, NOS $(n=215,35 \%)$. Of the 196 AITL cases extensively investigated for CD10, TFH markers (PD1, CXCL13), CD21/CD23 follicular dendritic cells (FDC) and EBV, the initial diagnosis was recorded in 178 cases as: AITL in 155 cases $(155 / 178=87 \%)$, PTCL, NOS in 21 cases $(12 \%)$, and intermediate between PTCL, NOS and AITL (PTCL, NOS/AITL) in 2 cases, indicating the impact of additional stainings for the diagnosis of AITL. The 107 PTCL, NOS cases also extensively immunostained included 9 follicular variant of PTCL, NOS; 8 PTCL, NOS/ AITL cases; 5 cases intermediate between PTCL, NOS and ALK-negative ALCL (of which 2 had been diagnosed as such and two as PTCL,NOS), and 85 cases truly unspecified. Of these, 60 had been initially diagnosed as PTCL, NOS; 2 as PTCL, NOS/AITL; 4 as ALK-negative ALCLs and 1 as ENKTL.

Conclusions: This study based on two independent large cohorts of non-cutaneous PTCLs highlights AITL as the most prevalent entity in Western Europe. It shows that extensive studies including investigation for CD10, TFH markers, FDC and EBV can at least partly contribute to the reclassification of some PTCL, NOS within the AITL category.

\section{LYS006}

Recurrent mutations in TET2, IDH2 and DNMT3 genes regulating DNA methylation in Peripheral T-Cell Lymphomas correlate with TFH-like features and clinical parameters

François Lemonnier ${ }^{1}$; Lucille Couronné ${ }^{2}$; Marie Parrens ${ }^{3}$; Jean-Philippe Jais ${ }^{4}$; Marion Travert ${ }^{5}$; Laurence Lamant ${ }^{6}$; Olivier Tournillac ${ }^{7}$; Thérèse Rousset ${ }^{8}$; Bettina Fabiani ${ }^{9}$; Antoine Martin ${ }^{10}$; Robert Cairns ${ }^{11}$; Tak Mak ${ }^{11}$; Christian Bastard $^{12}$; Olivier Bernard ${ }^{2}$; Laurence de Leval ${ }^{13}$; Philippe Gaulard $^{5}$

(1) Departement de Pathologie, Hopital Henri Mondor, Creteil, France (2) INSERM U985, Institut Gustave Roussy (3) Département de Pathologie, Hôpital Pessac (4) Département de Statistiques. Hôpital Necker (5) Département de Pathologie \& INSERM, U955, Hôpipal Henri Mondor (6) Département de Pathologie, Hôpital Purpan (7) Service d'Hématologie, CHU (8) Départements de Pathologie, CHU Gui de Chauliac (9) Département de pathologie, Hôpital Saint-Antoine (10) département de pathologie, Hôpital avicenne (11) Campbell Family Institute for Breast Cancer Research at Princess Margaret Hospital (12) INSERM, U918, Centre Henri Becquerel (13) Institut de Pathologie. CHU Vaudois

Background: The pathogenesis of angioimmunoblastic Tcell Lymphoma (AITL) and peripheral T-cell lymphoma, not otherwise specified (PTCL-NOS) is unknown, but AITL and a proportion of PTCL-NOS are thought to derive from follicular helper lymphocytes (TFH). Recently, mutations of TET2, IDH2 and DNMT3, three genes regulating DNA methylation, have been identified in PTCL.

Aim of the study: To evaluate the frequency of mutations in TET2, IDH1-IDH2 and DNMT3 genes in a large series of PTCL, and to correlate the mutational status with clinicopathological features.

Materials and methods: 190 PTCL lymphoma samples were investigated for TET2, IDH2 and DNMT3A mutations by direct sequencing.

Results: 64/190 (34 \%) PTCLs harbored TET2 mutations, including 40/86 (47 \%) AITL, 22/58 (38 \%) PTCL-NOS, 2/ $10(20 \%)$ enteropathy-associated T-cell lymphoma, but no anaplastic large cell $(0 / 18)$, extranodal NK/T $(0 / 12)$ and hepatosplenic (0/6) T-cell lymphomas. TET2 mutations 
strongly correlated with TFH derivation, occurring at higher frequency in AITL and TFH-like PTCL-NOS (PTCL-NOS with TFH markers and/or immunohistochemical features reminiscent of AITL) than in other PTCL entities ( $49 \%$ vs $12,5 \%, p<0.0001)$. DNMT3A and IDH2 were found mutated in 28/163 (17\%) and 8/31 (26\%) PTCLs, respectively (all in AITL and/or PTCL-NOS). TET2 and DNMT3A mutations strongly correlated as 24/61 (39 \%) TET2 mutated patients and only 4/102 (4\%) TET2 wild-type patients harbored DNMT3A mutations $(p<0.0001)$. In addition, IDH2 and TET2 mutations were not mutually exclusive as 6/8 IDH2 mutated samples were also TET2 mutated. Among the 144 AITL or PTCL-NOS patients, TET2 mutations associated with advanced stage disease, numerous involved extranodal sites, B symptoms, thrombocytopenia, high IPI and PIT scores and a shorter progression free survival $(p=0.04)$.

Conclusion: TET2, IDH2 and DNMT3A mutations are frequent in TFH-derived PTCLs, with TET2 mutations associated with aggressive disease. The involvement of these three genes regulating chromatin remodelling, suggests an important role of epigenetic changes in T-cell lymphomagenesis.

\section{LYS007}

PD1 expression in primary cutaneous small/medium CD4+ T-cell lymphomas distinguishes tumors with different clinical behavior

Mileyka Herrera $\mathrm{C}^{1}$; Adriana Garcia Herrera ${ }^{1}$; Ignasi Pau ${ }^{1}$; Davide Soldini ${ }^{1}$; Alexandra Valera ${ }^{1}$; Antonio Martinez ${ }^{1}$; Armando Lopez-Guillermo ${ }^{1}$; Teresa Estrach $^{1}$; Elias Campo ${ }^{1}$

\section{(1) Hospital Clinic i Provincial}

Background: Primary cutaneous small/medium CD4+ Tcell lymphoma (PCSM-TCL) is a provisional entity, which relationship to reactive lesions is not clear, and also may include several clinico-pathological categories. In a previous study, we identified three groups of PCSM-TCL: Group 1 included cases with indolent clinical course, groups 2 cases with aggressive clinical behavior and groups 3 multifocal-relapsing tumors with eosinophilia. The relationship between these subsets of lesions is not well understood but their distinction is important for the management of the patients. Recent studies have identified the expression of PD1 as a constant phenotypic feature of PCSM-TCL with indolent clinical behavior.

Materials and methods: We studied PD1 expression in 12 atypical cutaneous lymphoid T-cell hyperplasia (ATCH) defined as CD4+ small cell lymphoproliferative lesions without aberrant T-cell phenotype or evidence of clonality and 33 PCSM-TCL, 22 of group 1, 8 of group 2 and 3 of group 3. All these lesions had an aberrant T-cell phenotype and/or TCR clonal rearrangement. All the patients in group 2 had progressive disease resistant to therapy or systemic dissemination.

Results: Multiple clusters of PD1+ cells were seen in 11/12 (92\%) ATCH, and in 19/22 (86 \%) PCSMTL of group 1, 1/ $8(13 \%)$ of group 2 , and $0 / 3$ of group 3 . Relapsing lesions in tumors of the group 1 were seen in 2/3 PD1-negative but only in 4/19 PD1-positive lesions. Aggressive tumors in group 2 had lower CD8- cells and higher Ki67 proliferative index.

Conclusions: PD1 is expressed in most PCSM-TCL with indolent behavior and ATCH suggesting that these two categories may belong to the same spectrum of lesions. On the contrary, almost all tumors with aggressive clinical behavior were PD1 negative suggesting that they may correspond to a different entity, probably related to a cutaneous presentation of peripheral T-cell lymphoma, NOS.

\section{LYS009}

Intravascular ALK-negative systemic anaplastic large cell lymphoma with localized cutaneous involvement and an indolent clinical course: toward recognition of a distinct clinicopathologic entity

Ryan Metcalf ${ }^{1}$; Bashey Sameer ${ }^{2}$; Youn Kim ${ }^{1}$; Dita Gratzinger ${ }^{2}$

(1) Stanford University Medical Center (2) Stanford University

Background: Anaplastic large cell lymphoma (ALCL) is a T-cell lymphoma whose prognosis hinges upon its distribution (systemic or primary cutaneous) and, in systemic ALCL, the presence or absence of anaplastic lymphoma kinase (ALK) expression. Intravascular large cell lymphoma was first recognized in the 1980 's, with a majority of reported cases since then being B-cell in origin. Among intravascular large non-B-cell lymphomas, there are multiple reports of often EBV + and highly aggressive cytotoxic T or NK lymphomas. Intravascular involvement is thought to represent de facto evidence of systemic disease. Intravascular ALCL, whether ALK-positive or negative, is extraordinarily rare and, when it presents in the skin, raises the question of whether this entity will behave in the aggressive manner of intravascular $\mathrm{T}$ cell lymphoma or in the indolent manner of primary cutaneous ALCL.

Case report: An 86-year old man originally presented in September 2008 with a left shin $6 \times 3.5 \mathrm{~cm}$ red-purple slightly tender plaque that had been present for approximately 1.5 years. Punch biopsy revealed nests of epithelioid cells with central necrosis within apparent dermal lymphovascular spaces; no extravascular or epidermal infiltrates were 
present. Immunohistochemistry was negative for CD20 and PAX5 but was positive for $\mathrm{CD} 3$ and $\mathrm{CD} 30$; further workup was negative for CD56, ALK, EMA, EBV by ISH, TIA-1 and granzyme. The vascular spaces were CD34+ and D2-40 positive, identifying them as lymphovascular channels. PET imaging and physical exam did not show significant lymphadenopathy or evidence of visceral involvement. The patient also denied B symptoms. Ultimately, he was treated with local radiotherapy with a complete response and lost to follow-up for 3.5 years, when he re-presented with a deep purple nodule $(1.5 \times 1.5 \mathrm{~cm})$ with a surrounding violaceous, thick, indurated nodulo-plaque $(3.5 \times 4 \mathrm{~cm})$ with vascular prominence on the lateral lower left leg just outside the prior radiation field. Again, there were no B symptoms and PET imaging and physical exam were negative for systemic involvement.

Literature review: We identified two prior single patient case reports of intravascular ALK-negative ALCL presenting in the skin (cIALC), one single patient case report of ALK+ intravascular ALCL presenting in the skin, and 5 case reports, encompassing 8 patients, of EBV+ and/or cytotoxic intravascular large T or NK lymphoma presenting in the skin (cIT/NKL). The single case of ALK+ intravascular ALCL presenting in the skin presented in the breasts of a 33 year old woman who had extensive nodal involvement and was treated with chemotherapy; follow-up was not available. The 8 patients with cIT/NKL presenting in the skin were older adults (median age 63, range 40-71), commonly presenting with reddish plaques on the trunk and/or extremities. 6 of 7 tested cases were EBV+ by in situ hybridization; 5 of 8 were CD56+; and 7 of 8 were TIA- 1 positive. Notably, 2 of 5 tested cases showed at least some CD30 reactivity. These patients commonly had systemic involvement at presentation ( 4 of 5 for whom data were available) and followed a generally clinically aggressive course, with median reported survival of 6.5 months and 5 of 8 patients dead of disease at last follow-up. The 3 patients with ALK-negative cIALCL were also older adults (median age 70 , range 47 to 83 ) presenting with red to violaceous non-pruritic plaques on the leg, scalp and back, respectively. CD56, EBV ISH, and TIA-1 data were available for two of three cases each and were all negative. Involvement was skin-limited in two cases, with PET + regional and systemic adenopathy in a third case. The median survival of the two skin-limited ALK-negative IALCL cases with available follow-up was 69.5 months, and all 3 patients were alive with disease at last follow-up. The difference in survival between IT/NKL and the two cases of IALCL with known outcome is statistically significant by the two-tailed MannWhitney $\mathrm{U}$ test $(p=0.037)$.

Conclusions: While the numbers are small and more comprehensive studies are clearly needed, it does appear that a subset of cutaneous intravascular large $\mathrm{T}$ cell lymphomas, namely IALCLs that show characteristic anaplastic morphology, strong diffuse CD30 staining, and are negative for ALK as well as EBV, CD56, and cytotoxic markers may show an indolent course that is markedly different from that of the more commonly-reported aggressive cIT/NKLs. We recommend that all cases of cutaneous intravascular ALCL be evaluated for EBV by in situ hybridization and CD56 and TIA-1 by immunohistochemistry, as CD30+ cases of cIT/NKLs have been reported. If these stains are negative and clinical staging is negative, the possibility of an indolent clinical course should be noted, opening up consideration for radiation or other localized therapy in elderly patients who may not be good candidates for aggressive systemic chemotherapy.

\section{LYS010}

ALK-positive anaplastic large cell lymphoma limited to the skin: clinical, histopathological and molecular analysis of 6 pediatric cases- a report from the ALCL99 study

Ilske Oschlies ${ }^{1}$; Jasmin Lisfeld ${ }^{2}$; Laurence Lamant ${ }^{3}$; Atsuko Nakazawa ${ }^{4}$; Emanuele SG d'Amore ${ }^{5}$; Ulrika Hansson ${ }^{6}$; Konnie Hebeda ${ }^{7}$; Ingrid Simonitsch-Klupp ${ }^{8}$; Jadwiga Maldyk $^{9}$; Leonhard Muellauer ${ }^{8}$; Marianne Tinguely ${ }^{10}$; Markus Stuecker $^{11}$; Marie-Cecile LeDeley ${ }^{12}$; Reiner Siebert ${ }^{13}$; Alfred Reiter $^{14}$; Laurence Brugieres ${ }^{12}$; Wolfram Klapper ${ }^{15}$; Wilhelm Wössmann ${ }^{16}$

(1) Department of Pathology, Hematopathology Section and Lymph Node Registry, University Hospitals SchleswigHolstein (2) Justus Liebig University (3) Centre Hospitalier Purpan (4) National Center for Child Health and Developement (5) UO di Anatomia Patologica (6) Sahlgrenska Universitetssjukhuset (7) Radbound University Nijmegen Medical Centre (8) Medical University Vienna (9) department of Pathology, Childrens Hospital (10) University Hospitals Zurich (11) Ruhr University Bochum (12) Institut Gustave Roussy (13) Institute of Human Genetics, Christian-Albrechts-University Kiel and University Hospital S-H (14) Department of Pediatric Hematology and Oncology (15) Institute of Pathology, Hematopathology Section and Lymph Node Registry, University Hospital S-H (16) Department of Pediatric Oncology, Ege University Faculty of Medicine

Background: The vast majority of systemic anaplastic large cell lymphomas (ALCL) in children harbor translocations involving the anaplastic lymphoma kinase (ALK) gene leading to expression of the chimeric ALK fusion proteins. Primary cutaneous ALCL is regarded as an ALK-negative variant and according to the WHO classification is part of the spectrum of 
primary cutaneous CD30-positive T-cell lymphoproliferative disorders.

Aim of the study: We reviewed skin limited CD30-positive lymphoproliferative disorders in pediatric patients included into the ALCL 99 multicenter study.

Materials and methods: $33 / 487$ pediatric patients registered within the ALCL 99 trial (1999 to 2006) presented with a skin limited CD30-positive lymphoproliferative disorder. In 23/33 patients, material for international histopathological review was available and the cases were studied for histopathological, immunophenotypical and clinical features as well as breaks within the ALK-gene.

Results: $5 / 23$ cases and one additional case -identified after closure of the trial- expressed ALK-protein. These six lymphomas were diagnosed as skin-limited ALK-positive ALCL. Complete staging excluded any other organ involvement in all children. ALK protein expression was demonstrated by immunohistochemistry in all these lymphomas and the presence of breaks of the ALK gene was genetically confirmed in five evaluable cases. The histopathological and clinical picture of these skin-restricted ALK-positive lymphomas was indistinguishable from that of primary cutaneous ALK-negative ALCL. Five children presented with a single skin lesion that was completely resected in four and incompletely resected in one. Three of these patients received no further therapy, two additional local radiotherapy and one chemotherapy. All children remain in complete remission with a median follow-up of 7 years ( 1 to 8 years).

Conclusion: We present 6 pediatric ALK-positive primary cutaneous ALCL. After thorough exclusion of systemic involvement, therapy confined to local measures seems to be sufficient to induce cure.

\section{LYS011}

CD30 expression in peripheral T-cell lymphomas, not otherwise specified: is it relevant for the identification of distinct subgroups?

Bettina Bisig ${ }^{1}$; Laurence de Leval ${ }^{2}$; David S. Rickman ${ }^{3}$; Pierre Sujobert ${ }^{4}$; Christophe Bonnet ${ }^{5}$; Teresa Marafioti ${ }^{6}$; Georges Delsol ${ }^{7}$; Laurence Lamant ${ }^{7}$; Philippe Gaulard ${ }^{8}$

(1) Institut Universitaire de Pathologie, Lausanne, Switzerland (2) university institute of pathology lausanne (3) Department of Pathology and Laboratory Medicine, Weill Cornell Medical College (4) Department of Pathology, hôpital Henri Mondor (5) Department of Hematology, CHU Sart Tilman (6) Department of Histopathology, University College Hospital (7) Department of Pathology, CHU Purpan (8) Département de pathologie, hopital Henri Mondor

Background: Peripheral T-cell lymphomas, not otherwise specified (PTCLs, NOS) constitute a heterogeneous group of clinically aggressive neoplasms with indistinct borders, encompassing all mature T-cell lymphomas not fulfilling the diagnostic criteria of more specifically defined entities. At the molecular level PTCLs, NOS are heterogeneous. In a previous gene expression profiling (GEP) study, we found by unsupervised analysis that PTCLs, NOS clustered according to $\mathrm{CD} 30$ expression, and showed that $\mathrm{CD} 30$ positive (CD30+) PTCLs, NOS had a reduced expression of genes involved in T-cell activation (including CD28, CD52, CD69) and signal transduction following T-cell receptor (TCR) ligation (such as Lck, Fyn, Itk) in comparison to $\mathrm{CD} 30$-negative (CD30-) tumors. The purposes of the present work were (1) to extend this molecular characterization of PTCLs to include anaplastic large cell lymphoma (ALCL) cases, and more specifically to explore the molecular relationship between CD30+ PTCLs, NOS and anaplastic lymphoma kinase-negative (ALK-) ALCLs; (2) to validate GEP findings at the protein level; (3) to analyze patients' outcome in the above mentioned subgroups of tumors.

Materials and methods: We performed gene set enrichment analysis (GSEA) based on published GEP datasets from two previous studies (Blood 2007;109(11):4952-63 and Blood 2007;109(5):2156-64). A series of 80 PTCLs, comprising 36 PTCLs, NOS (18 CD30+ and 18 CD30-), 15 ALK- ALCLs and 29 ALK+ALCLs, mostly included in tissue microarray blocks, was subject to immunohistochemistry for assessment of 21 proteins, selected among those most differentially expressed. They included molecules involved in TCR signaling (TCR $\beta F 1$, CD3, ZAP-70, Lck, Fyn, Itk) cell-surface antigens (CD69, CD52, ICOS), transcription factors (NFATc2, JunB, pSTAT3, C/EBP $\beta$, MUM1/IRF4) and cell cycle regulators (cyclin D3). Differences in immunostaining scores (scoring scale ranging from 0 to 12) between the PTCL subgroups were assessed and hierarchical cluster analysis was conducted. Clinical followup was recorded when available.

Results: GSEA demonstrated that CD30+ PTCLs, NOS, compared to CD30- tumors, were significantly enriched in ALK- ALCL-related genes (141 genes; $P=0.0415$ ). By immunohistochemistry CD30+ PTCLs, NOS differed significantly from CD30- samples, most notably by the downregulation of TCR signaling-associated molecules, while displaying important overlaps with other $\mathrm{CD} 30+$ PTCLs, particularly ALK- ALCLs. The expression of the proximal tyrosine kinases Lck, Fyn and Itk was largely conserved in CD30- PTCLs, NOS (medians of immunostaining scores: 7.5, 8.0 and 12.0), while it was mostly lost in all CD30+ PTCLs (medians: 0; $P<0.0001$ ). CD30+ PTCLs were also associated with downexpression of proteins involved in T-cell activation, comprising CD69, ICOS, CD52 and NFATc2 (medians for CD30- PTCLs, NOS: 3.0, 9.5, 2.0 and 10.0; medians for the whole group of CD30+ PTCLs: 0; 
$P<0.0001)$. JunB and MUM1/IRF4 were significantly upregulated in all CD30+ PTCL categories (medians for the whole group of CD30+ PTCLs: 10) compared to CD30PTCLs, NOS (medians: $0 ; P<0.003$ ). C/EBP $\beta$ and pSTAT3 were both highly upregulated in ALK+ALCLs (medians: 12) compared to the other PTCL categories (medians for C/EBP $\beta$ : $0-2 ; P<0.0002$; medians for pSTAT3: $0-5.5 ; P<$ 0.004). Only 2 markers (CD3 and pSTAT3) were discriminant between CD30+ PTCLs, NOS and ALK- ALCLs. Hierarchical cluster analysis of the immunohistochemical data confirmed significant overlaps among the various CD30+ entities, particularly between CD30+ PTCLs, NOS and ALK- ALCLs. Conversely, CD30- PTCLs, NOS clustered together as a separate group. Survival analyses showed a tendency for CD30- PTCLs, NOS to have a worse outcome (overall and progression-free survivals) compared to the CD30+ subgroups, although the differences were not significant.

Conclusions: This study demonstrates the existence of molecular and phenotypic similarities across the various CD30+ PTCL subgroups, particularly between CD30+ PTCLs, NOS and ALK- ALCLs. Conversely, significant differences are evidenced between CD30- and CD30+ PTCLs, NOS. These results suggest that two biologically distinct subgroups of PTCLs, NOS might be delineated by the expression of CD30. The putative clinical relevance of this segregation might be reinforced by the potential benefits of incorporating anti-CD30 immuno-conjugates into the treatment strategies of CD30+ PTCLs.

\section{LYS012}

\section{DDX21, a central gene for rRNA Biogenesis is differentially expressed in ALK+ALCL, and is important for proliferation and survival of ALK+ALCL cells}

Irina Bonzheim ${ }^{1}$; Martin Irmler $^{2}$; Julia Steinhilber ${ }^{3}$; JanineAlison Schmidt ${ }^{3}$; Margit Klier-Richter ${ }^{3}$; Sabine Schäfer ${ }^{1}$; Patrick Adam ${ }^{3}$; Falko Fend ${ }^{1}$; Leticia Quintanilla-Martinez ${ }^{1}$

(1) Institute of Pathology and Neuropathology, University Hospital Tuebingen, Eberhard-Karls-University (2) Experimental Genetics, Helmholtz Center Munich - German Research Center for Environmental Health, Ne (3) Institute of Pathology, University Hospital Tübingen, Germany

Background: We recently identified CEBP $\beta$ a leucine zipper transcription factor, as a crucial mediator of survival and transformation of ALK + ALCL cells. Performing gene expression profiling (GEP) studies, we identified several direct and indirect target genes of CEBP $\beta$. Due to the emerging role of CEBP $\beta$ in the control of ribosomal RNA (rRNA) biogenesis in adipogenesis, it was remarkable to find that the RNA helicase DDX21, an important regulator of rRNA biogenesis, was strongly regulated by $\mathrm{CEBP} \beta$.

Aim of the study: To specify the functions of DDX21 in ALK-CEBP $\beta$-mediated oncogenesis, we analyzed the effects of DDX21 knockdown in ALK+ALCL cells. The expression of DDX21 protein was further investigated in primary ALK+ and ALK- ALCL cases.

Materials and methods: GEP was performed in 4 ALK+ALCL cell lines with and without CEBP $\beta$ knockdown. Candidate genes were validated by RTqPCR and by Western Blot. Chromatin immunoprecipitation was done to analyse direct promoter binding of CEBP $\beta$ at potential binding sites. DDX2 1 silencing in SUDHL-1 was performed by transduction of a highly specific shRNA. Cell proliferation and viability assay (MTS) and apoptosis assay with Annexin V were performed. Production of $28 \mathrm{~S}$ and $18 \mathrm{~S}$ rRNA was investigated by metabolic labeling (32P) and autoradiography. Immunohistochemistry (IHC) was performed in 18 (10 ALK+, 8 ALK-) primary ALK+ and ALKALCL cases.

Results: The GEP study identified DDX21 as one of the genes strongly regulated by $С Е B P \beta$. Although we could not demonstrate a promoter interaction of CEBP $\beta$ with the DDX21 promoter binding sites, the possible role of DDX21 in cell growth and metabolism of ALK +ALCL cells was further investigated in SUDHL-1 cells. Efficient downregulation of DDX21 mRNA $(92 \%)$ and protein showed important growth retardation $(65 \%)$ and increased apoptosis and cell death (58 \%) in SUDHL-1 cells when compared to control cells. To demonstrate that these effects most probably were secondary to stop in rRNA biogenesis with the subsequent derangement of cell metabolism, we investigated whether processing of pre-rRNA was interrupted by silencing DDX21. Efficient DDX21 knockdown revealed that the processing of $32 \mathrm{~S}$ pre-rRNA was clearly delayed in the cells. The downregulation of DDX21 resulted in a $70 \%$ and $74 \%$ decrease in the levels of $28 \mathrm{~S}$ and $18 \mathrm{~S}$ rRNA, respectively after a $3.5 \mathrm{~h}$ chase compared to control cells, indicating that DDX21 is critical for $18 \mathrm{~S}$ and $28 \mathrm{~S}$ rRNA biosynthesis and maintenance of cell metabolism in ALK+ALCL cells. Additionally, DDX21 expression was investigated in ALCL primary cases by IHC. All ALK+ALCL cases showed a strong nucleolar overexpression of DDX21 in the tumor cells. In contrast, ALK- ALCL cases showed normal expression of DDX21 comparable to the reactive lymphoid cells.

Conclusions: DDX21 is an indirect downstream target of $\mathrm{CEBP} \beta$, which is involved in the regulation of rRNA biogenesis, and has a key role in the control of cell 
metabolism and cell growth. DDX21 similarly to CEBP $\beta$, is differentially overexpressed in ALK + ALCL primary cases. DDX21 protein overexpression in ALK +ALCL tumors most probably reflects the increased ribosomal biogenesis needed for cell growth and proliferation contributing to the ALK-mediated lymphoid transformation. Therefore, it might be a potential candidate for targeted therapy.

\section{$\underline{\text { LYS013 }}$}

Peripheral T-cell lymphomas not otherwise specified correspond to distinct mature T-cell populations basing on the global gene expression profile

Fabio Fuligni ${ }^{1}$; Claudio Agostinelli ${ }^{2}$; Maura Rossi ${ }^{1}$; Claudio Tripodo ${ }^{3}$; Antonio De $\mathrm{Leo}^{2}$; Clara Bertuzzi ${ }^{2}$; Davide Gibelini $^{4}$; Maria Rosaria Sapienza ${ }^{1}$; Maria Antonella Laginestra $^{2}$; Anna Gazzola ${ }^{2}$; Stefano A. Pileri ${ }^{2}$; Pier Paolo Piccaluga ${ }^{4}$

(1) Haemolymphopathology Unit - S. Orsola-Malpighi Hospital - University of Bologna - Italy (2) Hematopathology Section, Department of Hematology and Oncology "L. and A. Seràgnoli”, S. Orsola-Malp (3) Palermo University (4) Haemolymphopathology - S. Orsola-Malpighi Hospital University of Bologna, Italy

Background: Peripheral T-cell lymphomas not otherwise specified (PTCLs/NOS) are the commonest T-cell neoplasms. Recent studies suggested that PTCL/NOS subgroups might be identified according to correspondence to various cellular counterparts. So far, it was possible only to discriminate T-helper (TH) vs. T-cytotoxic (TC) tumors. Noteworthy, due to their typical aberrant phenotype, such distinction could be effectively performed only by gene expression profiling (GEP).

Materials and methods: We generated and analyzed GEP to define the counterparts of PTCLs/NOS, using 108 PTCLs/NOS by Affymetrix HG-U133.2.0-plus microarray ( $N=28 \mathrm{fresh} /$ frozen samples) and Illumina whole genome DASL assay $(N=80$ formalin fixed paraffin-embedded (FFPE)). Immunohistochemistry (IHC) on tissuemicroarrays (TMAs) including the same cases was used for candidate markers validation. The molecular signatures of T-cell subsets (Th1, Th2, Th17, T-follicular-helper/Tfh, Treg,

T-central-memory/Tcm, T-effector-memory/Tem, and Tcytotoxic/TC) were studied in the tumor samples using a bioinformatic approach based on previously validated cell classifiers.

Results: We found that $23 / 28$ fresh/frozen samples corresponded to TH and $5 / 28$ to TC cells.
Moreover, the TH were more related to Tcm cells (14/23), than to Tem $(5 / 23)$ with 4 unclassified samples. When further signatures were tested we classified PTCLs as Th1 (16/23), Th2(1/23), Tfh(2/23), Treg(2/23), Th17(0/23) with 2 unclassified.

Testing FFPE samples we could assign $66 / 80(83 \%)$ cases to a specific counterpart, with $42 / 80 \mathrm{TH}$ and $24 / 80 \mathrm{TC}$. Further classification assigned TH cases to $\operatorname{Tcm}(N=20), \operatorname{Tem}(N=7)$, $\operatorname{Th} 1(N=21), \operatorname{Th} 2(N=13), \operatorname{Tfh}(N=4)$ or $\operatorname{Treg}(N=2)$.

IHC confirmed the genomic data only for Tfh and Treg markers, not being available consistent IHC markers for Th1, Th2, Tcm and Tem. Conversely, it was inadequate to distinguish $\mathrm{TH}$ and $\mathrm{TC}$, due to lack of $\mathrm{CD} 4, \mathrm{CD} 8$ and other cytotoxic molecules.

Conclusions: We showed that PTCL/NOS heterogeneity is largely due to the correspondence to different counterparts and confirmed our previous observation that GEP is probably more effective than IHC for the subclassification of these tumors.

\section{LYS014}

Small nucleolar RNA Expression Profiling identifies prognostic markers in Peripheral T-cell lymphoma

Pierre Brousset ${ }^{1}$; Valleron Wilfried ${ }^{2}$; Lamant Laurence ${ }^{3}$; Fataccioli Virginie ${ }^{4}$; Ysebaert Loic ${ }^{4}$; Gaulard Philippe ${ }^{4}$

(1) Toulouse, France (2) Inserm U1037 (3) Dpt of Pathology (4) Departement de Pathologie, Hopital Henri Mondor, Creteil, France

Background: Peripheral T-cell lymphomas (PTCL) are types of rare and heterogeneous non-Hodgkin's lymphoma (NHL) that, in general, are associated with a poor clinical outcome. Discovery of new prognostic tools is thus a current and major challenge.

Aim of the study: We focus our attention on the expression profiles of subclasses of non coding RNAs referred to as small nucleolar RNAs (snoRNAs) in a series of cases of PTCL of various types.

Materials and methods: A cohort of 122 cases of PTCL was collected from a multicentric T-cell lymphoma consortium (TENOMIC). We analyzed the expression of 80 small nucleolar RNAs (snoRNAs) using high-throughput quantitative PCR (Fluidigm). We demonstrate that snoRNA expression analysis may be useful in the diagnosis of some subtypes of PTCL and in the prognostication of both PTCLnot otherwise specified (PTCL-NOS) $(n=26)$ and angioimmunoblastic T-cell lymphoma (AITL) $(n=46)$ patients treated by chemotherapy.

Results: As with microRNAs, we noticed a global downregulation of snoRNAs in tumor cells compared to their 
normal counterparts. The snoRNA signature was robust enough to differentiate anaplastic large cell lymphoma $(n=$ 32) from the other PTCLs. For PTCL-NOS and AITL, we obtained two distinct prognostic signatures with a reduced set of 3 genes. Of particular interest was the prognostic value of HBII-239 snoRNA which was significantly overexpressed in cases of AITL and PTCL-NOS with favorable outcomes. It turned out that HBII-239 was processed into microRNA-768 3p whose expression paralleled that of the corresponding snoRNA. Bioinformatics followed by in vitro experiments showed that microRNA-768 directly targeted histone deacetylase 2 (HDAC2) gene.

Conclusions: Overall, we demonstrate that snoRNA expression profiles may have prognostic significance in PTCL, offering new tools for patient care and follow-up.

\section{LYS015}

\section{Integrative genomic and transcriptomic analysis of $\gamma / \mathcal{\delta}$} hepatosplenic T-cell lymphoma

Julio Finalet Ferreiro ${ }^{1}$; Leila Rouhi ${ }^{1}$; Helena Urbankova ${ }^{1}$; S. Shetty $^{1}$; B. Roland ${ }^{1}$; L. Krenacs ${ }^{1}$; Thomas Tousseyn ${ }^{2}$; Christiane De Wolf-Peeters ${ }^{2}$; Pascale De Paepe ${ }^{2}$; Peter Vandenberghe $^{1}$; Stein Aerts ${ }^{1}$; Jan Cools ${ }^{1}$; Iwona Wlodarska ${ }^{1}$

(1) Center for Human Genetics, KU Leuven, Leuven, Belgium (2) Translational Cell and Tissue Research, KU Leuven, Belgium

Background: $\gamma / \delta$ Hepatosplenic T-cell Lymphoma $(\gamma / \delta$ HSTCL) is a rare neoplasm derived from cytotoxic $\gamma / \delta$ Tcells characterized by an aggressive clinical course, chemoresistance and short survival. The lymphoma is hallmarked by isochromosome $7 \mathrm{q}[i(7)(\mathrm{q} 10)]$, an aberration resulting in loss of the short arm (p) and duplication of the long arm (q) of chromosome 7. Recently, we have collected 3 cases of $\gamma /$ $\delta$ HSTCL with ring $7[\mathrm{r}(7)]$ showing gain of the $7 \mathrm{q}$ sequences. Molecular consequences of chromosome 7 aberrations in $\gamma / \delta$ HSTCL remain elusive.

Aim: Our study aims at identification of molecular events underlying pathogenesis of $\gamma / \delta$ HSTCL.

Methods: Four cases with i(7)(q10), including DERL2, a $\gamma / \delta$ HSTCL-derived cell line, and 3 cases with $r(7)$ were analyzed by FISH and aCGH (Agilent 244 k). Further analysis included global expression profiling (GEP) using the Affymetrix Human Genome U133 Plus 2.0 Array (3 cases), high-throughput sequencing (Roche 454 GS-FLX) (DERL2) and paired-end RNA-sequencing (Illumina HighSeq2000) (DERL2 and 2 cases). Data were analyzed using the software ArrayStudio (Omicsoft Corporation). To increase statistical significance, additional cases of $\gamma / \delta$ HSTCL, peripheral T-cell lymphoma and normal controls were retrieved from the public databases.

Results: Array CGH and FISH analysis of 3 cases with r(7) confirmed gain/amplification of the $7 \mathrm{q}$ sequences and showed a constant loss of sequences distal to TCRG (7p14.1) and TCRB (7q34). The later finding indicates for the involvement of TCR recombination machinery in a generation of ring 7. Genomic profiling of all 7 analyzed cases allowed definition of the commonly deleted and gained regions which were located at 7 p14p22.3 (37 Mb) and $7 \mathrm{q} 21(24 \mathrm{Mb})$, respectively. One case displayed a biallelic microdeletion at $7 \mathrm{p} 21.3(1 \mathrm{Mb})$ covering 3 coding genes; however, high throughput sequencing of this region did not identify relevant coding mutations in DERL2. Comparison of GEP of $3 \gamma / \delta$ HSTCL and 3 normal spleens identified 1757 differentially expressed genes, including 45 downregulated and 1712 upregulated. Top biological functions of these genes include cellular development, cell growth, cell death and inflammatory response. Expression of T-/NK-cell markers confirms T-cell origin of this lymphoma. Integrative analysis identified only one downregulated gene at $7 \mathrm{p} 14 \mathrm{p} 22.3$ (MEOX2) and 31 upregulated genes in the commonly gained $7 \mathrm{q} 21$ region, of which ABCB1, CDK6, PIK3 complex and MCM7 showed the highest expression. These genes play important roles in a drug efflux mechanism, cell cycle regulation and control of chromosome remodeling, respectively. Similar results were obtained when additional cases from public databases were included in the analysis. RNA-sequencing analysis performed on DERL2 and $2 \gamma / \delta$ HSTCL cases confirmed upregulation of the $7 \mathrm{q} 21$ genes and showed lack of coding mutations in genes located in the commonly deleted $7 \mathrm{p}$ region, including MEOX2. Further analysis of RNAsequencing data is ongoing.

Conclusions: We showed that $\gamma / \delta$ HSTCL is characterized by a common loss of the $37 \mathrm{Mb}$ region at $7 \mathrm{p} 14 \mathrm{p} 22.3$ and gain of the $24 \mathrm{Mb}$ area at 7q21. So far, the postulated tumor suppressor gene at $7 \mathrm{p}$ was not identified; however, we found a set of gained/upregulated genes at $7 \mathrm{q} 21$ which may contribute to development, progression and chemoresistance of $\gamma / \delta$ HSTCL.

\section{LYS016}

CD8aa expression on CD4 lymphocytes of the colonic lamina propria defines a new subpopulation of human mucosal regulatory $T$ cells

Céline Bossard ${ }^{1}$; Guillaume Sarrabayrouse ${ }^{2}$; Anne Jarry ${ }^{3}$; Joé-Marc Chauvin ${ }^{2}$; Juliette Desfrançois ${ }^{2}$; Guillaume Meurette ${ }^{4}$; Laurent Potiron ${ }^{5}$; Francine Jotereau ${ }^{2}$

(1) CHU Nantes, France (2) INSERM U892 (3) EA 4273 Biometadys (4) Service de Chirurgie Digestive et 
Endocrinienne, CHU Nantes (5) Service de Chirurgie Digestive, clinique Jules Verne

Background: The mucosal immune system of the gut is a complex network including epithelial cells, and numerous innate and adaptive immune cells, including regulatory $\mathrm{T}$ cells (Treg). Previous studies in mice reported the presence of IL-10 secreting CD4+CD8aa + Treg in the gut epithelium. In the human gut mucosa, CD4+CD8aa $+\mathrm{T}$ cells have also been detected, but in the lamina propria. Furthermore, the phenotype and functions of these cells remain unexplored and their in vivo significance is unknown.

Materials and methods: We isolated $\mathrm{T}$ cells from normal colonic mucosa, and from inflamed colonic mucosa of patients with inflammatory bowel disease (IBD). We analyzed the density, the phenotype, and functions of CD4+CD8aa + T-cells using flow cytometry, as well as cell cultures.

Results: Here we show that CD4+CD8aa $+\mathrm{T}$ cells are present in the normal colonic mucosa with a higher density in the lamina propria (mean $5,3 \%+-3,6 \%$ of CD3 + cells) than in the epithelium $(1,5 \%+/-0,3 \%)(p<0,05)$. These cells are TCRa + T-cells presenting a polyclonal repertoire. They highly express markers of activated lymphocytes (CD25, CD69, HLA-DR), adhesion molecules (LFA1, LFA3, ICAM1), and are characterized by a regulatory phenotype (LAG3+, CTLA4+, GITR+) but lack Foxp3. Upon proper stimulation, they can secrete Th1 cytokines (TNFa, IFNg) as well as IL10. Importantly, they inhibit the proliferation of allogeneic $\mathrm{CD} 4+\mathrm{T}$ cells and the maturation of dendritic cells, in vitro. $\mathrm{CD} 4+\mathrm{CD} 8 \mathrm{aa}+\mathrm{T}$ cells are also present in inflamed mucosa, but with a lower density than in normal mucosa, suggesting a role of these cells in the maintenance of colonic mucosa homeostasis.

Conclusions: Our study describes for the first time a new subpopulation of $\mathrm{T}$ cells with regulatory phenotype and function in the human colonic mucosa. These results represent a new step in our understanding of the colonic mucosa immunity.

\section{$\underline{\text { LYS017 }}$}

\section{Indolent T-cell lymphoproliferative disease of the gastrointestinal tract: report of seven cases}

Anamarija Perry ${ }^{1}$; Roger Warnke ${ }^{2}$; Qinglong $\mathrm{Hu}^{3}$; Andreas Rosenwald ${ }^{4}$; Phillipe Gaulard ${ }^{5}$; Christiane CopieBergman $^{6}$; Eva Geissinger ${ }^{7}$; Huan-You Wang ${ }^{8}$; Erik Ranheim $^{9}$; Dennis Weisenburger ${ }^{1}$; Elaine Jaffe ${ }^{10}$; Wing Chan ${ }^{1}$

(1) University of Nebraska Medical Center (2) Stanford University Medical Center (3) Alegent Healthcare (4)
Institute of Pathology, University of Würzburg (5) Hôpital Henri Mondor (6) Département de Pathologie, Inserm U955 (7) University of Würzburg (8) University of California (9) University of Wisconsin (10) National Cancer Institute

Background: Primary T-cell lymphoma of the gastrointestinal tract is rare and generally an aggressive disease. Two variants are recognized, type I associated with an underlying enteropathy (primarily celiac disease), and type II which is usually not associated with enteropathy. However, a few reports in the literature have described clonal T-cell proliferations in the gastrointestinal tract with an indolent clinical course. Recently, NK-cell enteropathy was recognized as an indolent proliferation of NK-cells with a good prognosis. Herein, we report 7 cases of gastrointestinal involvement by an indolent $\mathrm{T}$ cell lymphoproliferative disease.

Aim of the study: The aim of the study is to describe the clinical, morphological, immunophenotypic and molecular features of 7 cases of indolent T-cell lymphoproliferative disease of the gastrointestinal tract.

Materials and methods: Gastrointestinal biopsies, molecular studies and clinical information from 7 patients were reviewed. Immunoperoxidase stains were performed on formalin-fixed, paraffin-embedded tissue sections using antibodies against $\mathrm{CD} 2, \mathrm{CD} 3, \mathrm{CD} 4, \mathrm{CD} 5, \mathrm{CD} 7, \mathrm{CD} 8$, CD56, TIA-1, granzyme B, TCR-beta F1, TCR-gammadelta, and Ki67 according to standard protocols. In situ hybridization for EBV-encoded RNA (EBER) was also performed. Molecular analysis for T-cell receptor gamma chain gene rearrangement was performed using the polymerase chain reaction on formalin-fixed, paraffin-embedded tissue according to established protocols for each participating institution.

Results: Among the 7 patients, 6 were males and one was female. The patients were relatively young with a median age of 38 years (range, 31 to 52 years). The most common presenting symptom was diarrhea (6/7 patients), with or without abdominal pain, mimicking inflammatory bowel disease. In addition to diarrhea, one patient had vomiting and another had dyspepsia and drenching night sweats. One patient presented with oropharyngeal ulcers and rectal bleeding. The sites of involvement included the oral cavity ( 1 patient), esophagus ( 1 patient), stomach ( 2 patients), small intestine ( 3 patients), and colon (4 patients). Two patients had widespread involvement including the esophagus, stomach, small intestine, and colon in one patient, and the oral cavity, small intestine and colon in another patient. Clinical staging showed no evidence of disease outside of gastrointestinal tract in all 7 patients. Three patients received no therapy, and one patient received only antibiotics for $\mathrm{H}$. pylori eradication. Three patients were treated aggressively, one with multiple courses of chemotherapy, one with 
chemotherapy, prednisone and sulfasalazine, and one with extensive bowel resection and TNF inhibitors. The median follow-up was 60 months (range, 6 to 168 months). At the time of last follow-up, 6 patients were alive with disease, and one patient was alive without disease. Histologically, all cases showed a similar dense lymphoid infiltrate in the mucosa/lamina propria composed predominantly of small, monotonous lymphoid cells with slightly irregular nuclei and clear cytoplasm. Frequently, eosinophils were admixed, but intraepithelial lymphocytes were few. The vast majority of the cells were T-cells and in 6 cases the infiltrate was CD3 +/CD8+, whereas one case was CD3+/CD4+. There was loss of CD5 in $1 / 7$ cases, and CD 7 in $3 / 6$ cases tested. All 6 CD8+ cases expressed TIA-1, whereas the one CD4+ case was TIA1-negative. Granzyme B was negative in all 5 cases tested, and CD56 was negative in all 7 cases. All 5 cases tested were positive for TCR-beta F1 and negative for TCRgamma-delta. Ki-67 was performed in 5 cases and all showed low proliferation (5-10\%). EBER was negative in all 6 cases tested, and all 7 cases had a clonal T-cell receptor gamma chain gene rearrangement.

Conclusions: We present a unique group of patients with an indolent T-cell lymphoproliferative disease confined to the gastrointestinal tract. Most of patients were young males presenting with diarrhea, clinically mimicking inflammatory bowel disease. Four patients had a long clinical course with no lymphoma therapy, and the infiltrate was unresponsive to chemotherapy in the other 2 patients. These lesions should be recognized since they can are easily be mistaken for intestinal T-cell lymphoma, which may lead to unnecessary or aggressive therapy.

\section{LYS018}

\section{Type II enteropathy-associated T-cell lymphoma: report of a european series}

Laurence de Leval ${ }^{1}$; Marie Parrens ${ }^{2}$; Brigitte Bouchindhomme ${ }^{3}$; Anne Moreau ${ }^{4}$; Céline Bossard ${ }^{4}$; Thierry Molina ${ }^{5}$; Marie-Hélène Delfau'; Philippe Gaulard ${ }^{7}$

(1) University Institute of Pathology Lausanne (2) CHU Bordeaux (3) Pole Pathologie Lille (4) CHU Nantes, France (5) CHU Hotel Dieu (6) CHU Henri Mondor (7) Département de Pathologie, Hopital Henri Mondor

Background: Enteropathy-associated T-cell lymphoma is a neoplasm derived from intraepithelial intestinal $\mathrm{T}$ lymphocytes. In the $2008 \mathrm{WHO}$ classification, two variants of this overall rare disease are recognized: type I, most common and associated with celiac sprue, usually featuring pleomorphic large cell morphology with frequent CD30 expression, and type II, less common with infrequent association to celiac sprue, which is composed of monomorphic cells with CD56 expression. Since the original description of type II EATL by Chott et al. in 1998, few cases series have been reported, mostly from Asia. Uncertainties remain regarding its association to enteropathy (and hence questioning its designation), its derivation in terms of T-cell lineage ( $\alpha$ versus $\gamma / \delta$ T cells), its morphologic and immunophenotypic spectrum, and the distinguishing features with type I.

Aim of the study: to characterize type II EATL encountered in Western Europe

Materials and methods: Fifteen cases fulfilling the diagnostic criteria for type II EATL were retrieved from the files of the Pathology Departments of several centers in France (Creteil, Bordeaux, Lille, Nantes), Belgium (Liège) and Switzerland (Lausanne), including the consultation files of two authors (LdL, PG). The available clinical informations were collected. The macroscopic and histological features of the tumors and distant mucosa were assessed. Detailed immunophenotypic characterization was performed. The tumors were molecularly investigated for rearangements of the TCRG and TCRD by PCR-DGGE.

Results: The patients included 7 males and 8 females aged 41 to 85 with a median age of 73 . None had a history of celiac sprue. All tumors occurred in the small bowel and presented as a single tumor mass except in one patient who had two lesions. The tumors ranged in size from 4,5 to $22 \mathrm{~cm}$ and were all macroscopically ulcerated. All tumors were transmural with a predominantly mucosal extension in three of them. Perforation was mentioned in three of 12 cases. Epitheliotropism to the adjacent mucosa was a constant feature. Mesenteric fat infiltration was seen in 11 cases, where the neoplastic cells showed features of angiocentricity as well as perineural invasion. Cytologically, 12 cases were composed of small to medium-sized lymphoid cells with a monomorphic (9) or blastoid (3) appearance, while three cases encompassed large cell morphology. Distant normal mucosa was evaluable in 6 cases, of which 2 showed increased itraepithelial lymphocytes (IEL). The usual immunophenotype was: CD2- (5/14+) CD3+ (15/15+) CD4- (1/ 14+) CD5- (1/15+) CD7+ (13/13+) CD8+ (14/15+) CD56+ (11/15+) CD57- (11/11-) CD30- (11/11-). All cases has a cytotoxic immunophenotype with expression of TiA1 in 15/ 15 cases, and expression of granzymeB and perforin in 12/ 14 and 5/14 cases, respectively. Coexpression of CD20 was evidenced in 4/15 cases, of which one exhibited an extensive B-cell immunophenotype (CD19+ CD79a+OCT2+); all cases tested for PAX5 were negative. None of the cases expressed CD10 and Ki67 proliferation index ranged from $20 \%$ to $95 \%$ without correlation with cytological features. One case investigated for CD103 expression on frozen tissue was positive. All cases were EBV-negative in tumor cells. Nine cases expressed gamma-delta T-cell receptor (TCR) and biallelic TCRD rearrangement was demonstrated 
in $6 / 6$ of these cases with contributive molecular analysis. Two cases expressed alpha-beta TCR, of which one had a monoclonal TCRG rearrangement and noncontributive TCRD PCR analysis, and the other had no demonstrable clonality for both. One case coexpressed both TCRs and was not investigated molecularly. Three cases were negative for both TCRs by immunohistochemistry and two of them had biallelic TCRD rearrangement while one was clonal for TCRG and non-clonal for TCRD. In all T-cell clonality (TCRG and/or TCRD) was demonstrated in 11/12 investigated cases.

Conclusion: In this European series of type II EATL, the disease manifests as ulcerated tumors occurring in the small intestine of elderly individuals without sex predominance and without evidence of association with celiac sprue. Type II EATL presents a morphologic spectrum deviating from the classical small to medium sized monomorphic appearance to include cases with blastoid and/or large cell morphology, and frequent angiocentric features. The usual cell surface profile is CD2- CD3+ CD4- CD5- CD7+ CD8+ CD56+, with an activated cytotoxic immunophenotype (TIA1+ granzymeB+ perforin-) and not infrequent coexpression of CD20. Type II EATL is characterized by a common but inconstant gamma-delta $\mathrm{T}$ cell derivation reflected in a gamma-delta TCR or TCR-silent immunophenotype.

\section{LYS019}

Epstein-Barr virus positive NK/T-cell lymphoproliferative disease of childhood and teenagers. Different clinical and pathological spectrum

Carlos Barrionuevo $^{1}$; Socorro María Rodriguez-Pinilla ${ }^{2}$; Juan García $^{2}$; Santiago Montes-Moreno ${ }^{3}$; Sandro Casavilca ${ }^{2}$; Jaime Montes ${ }^{2}$; Miguel Ángel Piris ${ }^{3}$

(1) Instituto Nacional de Enfermedades Neoplásicas (INEN)

(2) Novartis (3) Pathology Department, Hospital Marques de Valdecilla, IFIMAV

Background: In the last classification of lymphoid neoplasms of the World Health Organization (2008) there are two provisional entities in relation to Epstein-Barr virus (EBV) positive T-cell lymphoproliferative disease of childhood; however, the spectrum of clinical manifestations and pathogenesis are still poorly understood.

Aim of the study: Herein, we report 23 of such cases from Peru, all with onset of disease in childhood or adolescence. They have complete clinical data, and histological, immnunohistochemistry, and molecular studies.

Materials and methods: Detailed clinical data were available in all cases. The morphology of each case was analyzed. Immnunohistochemistry staining, in-situ hybridization for EBV-encoded RNA (EBER), and B-cell and Tcell clonality studies were performed in all cases.

Results: There were 17 men and 6 women with a median age of 12 years (range: 1.4-36 years). Eleven of them fit into the category of hydroa vacciniforme-like lymphoma (HVLL), 3 had skin lesions similar to those observed in HVLL but without facial involvement, 3 had onset with systemic and nodal disease, 5 had regional nodal clinical presentation, and one had systemic/disseminated clinical presentation without lymphadenopathy. All cases with cutaneous lymphoma were characterized by infiltration of smallto-medium sized lymphocytes showing epidermotropism. The other cases showed a diffuse infiltration of intermediate-to-large pleomorphic cells. Vascultis and geographical areas of necrosis were seen in some of the cases. The immunophenotype showed a cytotoxic T or NK-cell lineage. In all cases the neoplastic cells showed EBER expression. Six of the cases showed clonal TCR rearrangement. After diagnosis the median survival was 6 months (range: 1-32 months).

Conclusions: These findings show a broad clinical and pathological spectrum for this EBV positive T-cell lymphoproliferative disease of childhood and teenagers, which may imply the existence of yet unknown pathogenic factors.

\section{LYS020}

Hydroa vacciniforme (HV) like-lymphoma in Mexican children

Leticia Quintanilla-Martinez ${ }^{1}$; Cecilia Ridaura ${ }^{2}$; Florian $\mathrm{Nagl}^{1}$; Georgia Alderete ${ }^{2}$; Peter Grube ${ }^{2}$; Marimar Sáez-deOcariz $^{3}$; Carola Durán-McKinster ${ }^{3}$; Carmen LomeMaldonado $^{4}$; Irina Bonzheim ${ }^{1}$; Falko Fend ${ }^{1}$

(1) Institute of Pathology, University of Tübingen, Germany, (2) Pathology and (3) Dermatology Departments, Instituto Nacional de Pediatria, Mexico, (4) Pathology Department, Instituto Nacional de la Nutricion, Mexico

Background: HV- like lymphoma (HVLL) was included in the 2008 WHO classification as a subgroup of EBV-positive T-cell lymphoproliferative disorders of childhood. It affects mainly children and adolescents from Asia and Latin America. The neoplastic cells are mostly cytotoxic CD8+ T-cells and rarely NK-cells. Patients present clinically with a papulo-vesicular rash in sun-exposed and non-exposed areas followed by ulceration and varioliform scars. The aim of this study was to analyze the clinicopathological and molecular features of 20 Mexican children diagnosed as HVLL. Material and methods: Paraffin sections were stained with antibodies against CD3, CD 4, CD8, CD20, 
CD30, CD56, BF1, LMP1, EBNA2 and TIA-1. EBVencoded small nuclear RNA (EBER) was analyzed by in situ hybridization. TCR $\gamma$ gene rearrangement was analyzed by PCR.

Results: 14 males and 6 females were included. The mean age of the patients at clinical presentation was 8 years (range 1-15 years). All presented with HV-like skin lesions in covered and sun-exposed areas characterized by edema, vesicles, erythema, ulcerations, crusts, and vacciniforme scars. Twelve patients $(60 \%)$ presented in addition with systemic symptoms such as fever, wasting, lymphadenopathy and hepatomegaly. Four patients showed hypersensitivity to mosquito bites (HMB). Thirteen patients have followup with median of 3 years (range 1 month -13 years). Two patients developed systemic lymphoma (2-4 years). 28 biopsies from 20 patients were analyzed. All biopsies showed essentially similar histological findings characterized by an atypical lymphoid infiltrate predominantly in the dermis that extended sometimes deep into the subcutaneous tissue. An intraepidermal blister was often observed. The infiltrate was mainly located around adnexa and blood vessels often with angiodestructive features. The intensity of the infiltrate and atypia of the lymphocytes varied from case to case. All cases were EBER+ but LMP1 was rarely expressed. EBNA2 was negative. Fourteen cases revealed a cytotoxic T-cell phenotype, three of which were CD3+, CD56-, CD4/ CD8 double negative and $\beta F 1$ negative suggesting a $\gamma / \delta$ phenotype, whereas six cases revealed an NK-cell phenotype (CD3+, 56+, TIA-1+), four of the latter had in addition hypersensitivity to mosquito bites. One patient had initially a T-cell neoplastic infiltrate; however, 3 years later he developed an NK-cell infiltrate associated with hypersensitivity to mosquito bites. CD30 was positive in 11 cases (6 Tcells, 5 NK-phenotype). Histologically, the NK cell lesions tended to infiltrate deep in the subcutaneous tissue with rimming of fat cells mimicking subcutaneous panniculitislike T-cell lymphoma. Thirteen of the 14 T-cell cases analyzed showed monoclonal TCR $\gamma$ gene rearrangement (93\%). The 6 cases with NK-cell phenotype were polyclonal. Patients were primarily treated with immunomodulating or immunosupressive therapy such as thalidomide, steroids and/or chloroquine. Thalidomide was the most effective drug, but the effect lasted for short periods of time and patients recurred with new skin lesions.

Conclusions: HVLL is an EBV-associated monoclonal disorder mostly with a CD $8+$ phenotype but also $\gamma / \delta$ and NKcell phenotype with a broad clinical spectrum, usually long clinical course, relative favourable response to immunomodulating drugs and low potential to progress to systemic disease. Cases associated with HMB show an NK-cell phenotype. Both lesions might present in the same patient, which suggests indeed that these two lesions are related.

\section{LYS023}

MYC status in concert with BCL2 and BCL6 expression predicts outcome in diffuse large B-cell lymphoma in the RICOVER-60 trial of the DSHNHL

Heike Horn ${ }^{1}$; Marita Ziepert ${ }^{2}$; Claudia Becher ${ }^{3}$; Thomas F.E. Barth $^{4}$; Heinz-Wolfram Bernd ${ }^{5}$; Alfred C. Feller ${ }^{6}$; Wolfram Klapper ${ }^{6}$; Michael Hummel ${ }^{7}$; Harald Stein $^{7}$; Martin-Leo Hansmann $^{8}$; Christopher Schmelter ${ }^{9}$; Peter Möller ${ }^{4}$; Sergio Cogliatti ${ }^{10}$; Michael Pfreundschuh ${ }^{11}$; Norbert Schmitz ${ }^{12}$; Lorenz Trümper ${ }^{13}$; Reiner Siebert ${ }^{14}$; Markus Loeffler ${ }^{2}$; Andreas Rosenwald ${ }^{9}$; German Ott $^{15}$

(1) Institut für klinische Pharmakologie (2) Institute for Medical Informatics, Statistics, and Epidemiology, University of Leipzig (3) Institute of Human Genetics, Christian-AlbrechtsUniversity Kiel and University Hospital Schleswig- (4) Institute of Pathology, University Hospital Ulm (5) Institute of Pathology, University Hospital Schleswig-Holstein, Campus Lübeck (6) Institute of Pathology, Hematopathology Section and Lymph Node Registry, University Hospital Schles (7) Institute of Pathology, Campus Benjamin Franklin, Charité Universitätsmedizin (8) Institute of Pathology, University Hospital Frankfurt (9) Institute of Pathology, University of Würzburg (10) Institute of Pathology, Kantonal Hospital St. Gallen (11) Medizinische Klinik I, Saarland University Medical School (12) Department of Hematology, Asklepios Klinik St. Georg (13) Department of Hematology and Oncology, Georg-August University (14) Institute of Human Genetics, Christian-Albrechts-University Kiel and University Hospital Schleswig-H (15) Department of Clinical Pathology, RobertBosch-Krankenhaus, and Dr. Margarete Fischer-Bosch Institut

Background: A rearrangement of MYC occurs in 5-10\% of diffuse large B-cell lymphomas (DLBCL) and seems to confer an increased risk to both $\mathrm{CHOP}$ and R-CHOP treated patients. Aim of the study: The prognostic relevance of rearrangements of MYC, BCL2 and BCL6 and protein expression for MYC, BCL2 and BCL6 was investigated in the large prospective randomized RICOVER trial of the German HighGrade Lymphoma Study Group.

Materials and methods: Paraffin-embedded tumor samples from 442 de novo DLBCL treated within the RICOVER study of the German High grade Lymphoma Study Group were stained for the expression of MYC, BCL2, and BCL6, and fluorescence in-situ hybridisation (FISH) was performed to detect breaks at MYC, BCL2 and BCL6 loci. Results were evaluated according to OS and EFS of the patients enrolled.

Results: Rearrangements of MYC, BCL2 and BCL6 were detected in $8.8 \%(36 / 407), 13.5 \%(52 / 384)$ and $28.7 \%$ 
(116/404) of DLBCL, respectively. Protein overexpression of MYC ( $>40 \%$ ) was encountered in $31.8 \%$ (90/283) of tumors, and was correlated with the presence of MYC translocations $(p<0.001) .79 .6 \%(313 / 393)$ and $82.8 \%(284 / 343)$ of tumors expressed BCL2 and BCL6, respectively. In univariate analyses, MYC translocations, MYChigh, BCL2high and BCL6low protein expression were associated with inferior survival (EFS, OS). In multivariate Cox regression modelling, protein expression patterns of MYC, BCL2 and BCL6, and MYC rearrangements predicted survival independent of the International Prognostic Index (IPI). Most important, an immunohistochemical and a FISH/IHC risk score could be defined that predicts prognosis in the RICOVER-60 trial and identifies a group of $15 \%$ of elderly patients with extremely poor prognosis within the high-risk IPI group.

Conclusions: MYC status in concert with BCL2 and BCL6 expression predicts outcome in the large German RICOVER trial and permits to identify a subset of $15 \%$ of elderly patients with dismal prognosis in the high-risk IPI group following treatment with R-CHOP.

\section{LYS024}

\section{Frequent detection of BRAF V600E mutation in histiocytic neoplasms}

Heounjeong $\mathrm{Go}^{1}$, Yoon Kyung Jeon ${ }^{2}$; Jooryung $\mathrm{Huh}^{3}$; Suk Jin Choi ${ }^{4}$; Yoo-Duk Choi ${ }^{5}$; Hee Jeong $\mathrm{Cha}^{6}$; Hyun-Jung $\mathrm{Kim}^{7}$; Gyeongsin Park ${ }^{8}$; Sooki Min ${ }^{9}$;i Eun Kim ${ }^{1}$

(1) Department of Pathology, Seoul National University Boramae Hospital, Seoul, Korea (2) Department of Pathology, Seoul National University Hospital (3) Asan Medical Center Ulsan college of Medicine (4) Department of Pathology, Inha University Hospital (5) Chonnam National University Medical School (6) Department of Pathology, University of Ulsan College of Medicine, Asan Medical Center (7) Department of Pathology, Inje University Sanggye Paik Hospital (8) Seoul St. Mary's Hospital, The Catholoic University of Korea (9) Department of Pathology, Hallym University Pyongchon Secret Heart Hospital

Background: Although BRAF mutation has been detected in a wide range of human cancers, in hematolymphoid neoplasms it has rarely been identified except in hairy cell leukemia and a very limited numbers of non-Hodgkin's lymphoma. Recent study revealed that the activating mutation BRAF V600E was found in more than half of Langerhans cell histiocytosis (LCH). Herein we examined the presence of BRAF mutation in a group of fibrohistiocytic neoplasms and compared the detection rate of BRAF V600E mutation in each disease group.
Materials and methods: A total of eighty-five cases of fibrohistiocytic tumors were reviewed from the archives of the 10 hospitals in Korea. Cases consisted of LCH $(n=27)$, histiocytic sarcoma (HS, $n=9$, including one Langerhans cell sarcoma), Rosai-Dorfman disease $(n=5)$, follicular dendritic cell sarcoma (FDC sarcoma, $n=24)$, interdigitating cell sarcoma $(n=1)$, inflammatory myofibroblastic tumor $(n=8)$, blastic plasmacytoid dendritic cell neoplasm $(n=7)$ and acute monocytic leukemia (AML-M5, $n=4$ ). DNA was isolated from the most representative sections of each paraffin block by manual dissection of tumor areas. BRAF V600E mutation analysis was performed by direct Sanger's sequencing and real time PCR using AnyplexTM BRAF V600E real-time detection v2.0.

Results: Among the fibrohistiocytic tumors, HS showed the highest BRAF mutation rate $(6 / 9,66.7 \%)$, followed by FDC sarcoma $(6 / 24,25 \%)$ and LCH $(6 / 27,22.2 \%)$. Other fibrohistiocytic tumors did not reveal BRAF mutation. In LCH and FDC sarcoma, there was no significant clinical predilection between tumors with BRAF mutation and tumors with wild type BRAF, regarding age, sex, site of involvement, etc. The sensitivity of direct sequencing and real time PCR was almost equal.

Conclusions: BRAF V600E mutation is not limited to LCH and more frequently detected in HS and FDC sarcoma. These findings suggest that BRAF pathway may contribute to the pathogenesis or malignant transformation of histiocytic neoplasm. Presence of BRAF mutation can be used as a reliable diagnostic marker of HS in the differential diagnosis with high grade lymphoma or other types of sarcoma.

\section{LYS025}

EBV-Bart-6-3p induces cell proliferation and escape of immunosurveillance in Burkitt lymphoma cell lines and primary tumours

Mohsen Navari ${ }^{1}$; Lorena Di Lisio ${ }^{2}$; Anna Onnis ${ }^{1}$; Nerea Martinez ${ }^{2}$; Maria Raffaella Ambrosio ${ }^{1}$; Giulia de Falco ${ }^{1}$; Eduardo Andres León ${ }^{2}$; Santiago Montes-Moreno ${ }^{2}$; Cristiana Bellan $^{1}$; Stefano Lazzi ${ }^{1}$; Emily A Rogena ${ }^{3}$; Walter Mwanda ${ }^{3}$; Miguel Angel Piris ${ }^{4}$; Lorenzo Leoncini ${ }^{1}$

(1) Department of Human Pathology and Oncology, University of Siena, Siena, Italy (2) Hospital U. Marqués de Valdecilla, IFIMAV, Cancer Genomics Laboratory (3) Department of Human Pathology, University of Nairobi (4) Pathology Department, Hospital Universitario Marques de Valdecilla

Background: Burkitt lymphoma (BL) is an aggressive Bcell lymphoma presenting in three clinical forms: endemic, 
sporadic and immunodeficiency-associated. More than $90 \%$ of endemic BL carry latent Epstein - Barr virus (EBV), though still no satisfactory explanation of how EBV participates in its pathogenesis is provided. In this regard, a role for virus-encoded microRNAs (miRNAs), which may target cellular genes, and therefore, dysregulate host cell gene expression, has been proposed.

Aim of the study: to compare EBV-pos and EBV-neg BL cases for miRNA expression profile, and to assess the role of viral miRNAs in Burkitt lymphomagenesis.

Materials and methods: MiRNA expression profiling of 18 BLs (six EBV-pos and twelve EBV-neg) was performed using Agilent ${ }^{\circledR}$ miRNA expression microarray technology. After array normalization, a t-test (limma) was used to compare EBV-pos and -neg samples. Target genes of both viral and human miRNAs were classified according to the Gene Ontology (GO). EBV-miR-BART6-3p was inhibited or expressed in $\mathrm{BL}$ cell lines and the expression level of its putative target genes was assessed using real-time PCR.

Results: Comparison between EBV-pos and EBV-neg BL cases identified human and viral miRNAs expressed in EBV-pos BL cases. We then focused on viral EBV-miR-BART6-3p and on two predicted target genes, PTEN and IL6ST (gp130). Ectopic expression of a synthetic miRNA determined a significant reduction of the expression of both PTEN and gp130, whereas inhibition of EBV-miR-BART6-3p resulted in marked up-regulation of both genes, thus suggesting that EBV-miR-BART6-3p is able to regulate the expression of PTEN and gp130 in human cell lines. These results were confirmed at protein level in EBV-pos and EBV-neg primary tumors $(p<0.001)$.

Conclusions: Collectively, our results suggest that ebvBART6-3p induces cell growth through downregulation of PTEN and escape of immunosurveillance through downregulation of gp130, giving evidence of an active role of EBV in BL pathogenesis.

\section{LYS026}

The role of antigenic stimulation in lymphoma growth: A novel mutated B-CLL subset expressing high affinity B-cell receptors specific for yeast and molds

Robbert Hoogeboom ${ }^{1}$; Kok van $\mathrm{Kessel}^{2}$; Frans Hochstenbach ${ }^{2}$; Thera Wormhoudt ${ }^{1}$; Roy Reinten ${ }^{1}$; Koen Wagner $^{3}$; Arnon Kater $^{4}$; Jeroen Guikema ${ }^{1}$; Richard Bende ${ }^{1}$; Carel van Noesel ${ }^{1}$

(1) Dpt. of Pathology, AMC (2) Dpt. of Medical Microbiology, UMCU, Utrecht (2) Dpt. of Medical Biochemistry, AMC (3) Dpt. of Histology and Cellbiology, AMC (4) Dpt. of Hematology, AMC, Amsterdam, the Netherlands

Background: The immunoglobulin repertoire of B-cell chronic lymphocytic leukemias (B-CLLs) is biased as compared to the repertoire of normal B-cell subsets. Approximately, $30 \%$ of B-CLLs can be classified in so-called homology subsets, based on the expression of stereotypic Bcell antigen receptor (BCR)-variable heavy chains ( $\mathrm{IgVH})$, most often paired with characteristic light (IgVL) chains. The occurrence of groups of B-CLL expressing highly similar BCRs strongly suggests a shared specificity and involvement of recurrent antigens in the development of BCLL. Direct proof for this notion however has not been provided.

Results: We identified four B-CLLs belonging to a novel V3-7 homology subset expressing highly similar, somatically hypermutated IgVHs coupled to near-identical IgVLs. Interestingly, all four B-CLLs shared two replacement mutations in their IgVH. In order to address their antigenic specificity, we recombinantly produced the BCRs of three patients belonging to this homology subset as soluble IgM (Subset-IgM), as well as of 26 control B-CLLs (ControlIgM). The Subset-IgM did not show any signs of auto/ polyreactivity in tissue-microarrays and were not found reactive by ELISA for well-known autoantigens, in contrast to IgM from unmutated control B-CLL. The Subset-IgM was tested by flow cytometry for reactivity with common pathogens and were found not to stain any of 19 major (grampositive and gramnegative) bacterial strains. By contrast, all three subset-IgM strongly stained several yeast species as well as spores and conidia of distinct mold species. None of the 26 Control-IgM showed this specificity. The human Subset-IgM antibodies also highly specifically stained fungi in clinical, formalin-fixed paraffine-embedded tissues.

Exhaustive epitope mapping analyses demonstrated that all three CLL-subset-derived BCRs have a high affinity for $\beta$ $(1,6)$-glucan, a main constituent in the fungal cell wall. Swopping of the subset-related IgVL with IgVL of control B-CLL abrogated the reactivity for $\beta-(1,6)$-glucan and reversion of the somatic mutations to germline nucleotides resulted in a lower affinity for $\beta-(1,6)$-glucan, indicating strong selection and affinity maturation of the B-CLL clones (or of their non-neoplastic precursor B cells) for $\beta-(1,6)$ glucan. Last but not least, primary cells of the subset B-CLL were induced to proliferate in vitro by $\beta-(1,6)$-glucan.

Conclusions: 1. We identified a novel B-CLL homology subset expressing highly similar BCRs with high affinity for $\beta$ - $(1,6)$-glucan, an epitope ubiquitously present in the cell walls of commensal fungi.

2. We provide first proof that different members of a hypermutated B-CLL homology subset indeed express BCRs of the same antigenic specificity.

3. We obtained evidence that BCRs of mutated B-CLL respond in vitro to their cognate antigen and transduce growth signals.

The findings provide strong support for the concept of antigen-driven lymphoma development. 


\section{LYS027}

T Lymphoblastic Leukemia Lymphoma with $t(8 ; 13)$ FGFR1 rearrangement

Antonio M. Hernandez ${ }^{1}$; Aurelia Meloni-Ehrig ${ }^{2}$; Antonio Planas ${ }^{3}$

(1) Ameripath Florida, Orlando, FL, USA (2) Ameripath /

Quest Cytogenetics (3) Hematopathologist

Background: 48 years old Male with elevated WBC and mild lymhadenopathy

Aim of the study: Explore possible origin of T cell neoplasm in the setting of a co-existing myeloproliferative neoplasm with biphenotypic potential.

Materials and methods: Flow cytometry, Cytogenetics, FISH, Morphology, \& IHC.

Results: $t(8 ; 13)$ was present in both myeloid elements in the bone marrow marrow and in the neoplastic $\mathrm{T}$ cells of the lymphoblastic lymphoma.

Bone Marrow involvement by the neoplastic T cells was limited, detected by IHC and microscopy but not flow cytometry. Conclusions: Transformational molecular events are not readily detectable by existing clinical laboratory techniques but cases such as this provide tissue for further study and pose questions for more insight.

\section{LYS028}

\section{Lennert's lymphoma- clinicopathological profile of 5 cases}

Parimal Sarda ${ }^{1}$; Rekha Pai ${ }^{2}$; Marie Therese Manipadam²; Sheila Nair ${ }^{2}$

(1) Christian Medical College, Vellore, India (2) Department of Pathology, Christian Medical College, Vellore

Background: Lennert's lymphoma is a rare variant of Peripheral T cell lymphoma. It is often confused with Hodgkin lymphoma due to the presence of Reed Sternberg (RS) like large cells and $\mathrm{T}$ cell receptor gene rearrangement (TCR) often helps to differentiate them. This is the first report of this entity from our country.

Aim of the study: To study the clinicopathological profile of Lennert's lymphoma along with describing the morphology and immunophenotypic features and determine the utility of TCR gene rearrangement in the diagnosis of Lennert's lymphoma.

Materials and methods: All cases diagnosed as Lennert's lymphomas during the period 1 Jan 2001 to 30 Aug 2011 were included in this study. H \& E stained slides \& immunohistochemistry results were analyzed. TCR gene rearrangement was performed on 3 cases.
Results: During the ten-year period there were 5 cases of Lennert's lymphoma diagnosed in our institution. This constituted $0.11 \%$ of all Non Hodgkin Lymphomas and $0.71 \%$ of all peripheral $\mathrm{T}$ cell lymphomas during that period. The five cases included 2 males and 3 females with age range from 27 to 68 years. Of these, clinical details and follow up was not available for two cases which were received from other hospitals. All cases showed complete effacement of lymph node architecture by diffuse infiltration of small lymphoid T-cells $(\mathrm{CD} 3+, \mathrm{CD} 4+, \mathrm{CD} 8+)$ and several clusters of epitheloid histiocytes and scattered large transformed cells (CD20-, CD30+, CD15-/+). TCR rearrangement was done in three patients on paraffin embedded tissue sections by PCR and showed the presence of a clonal $\mathrm{T}$-cell population in all three cases.

Conclusions: Lennert's lymphoma constituted $0.11 \%$ of all NHLs in our institution. Differentiation from classical Hodgkin lymphoma is sometimes difficult by morphology and immunohistochemistry alone and TCR gene rearrangement was extremely useful in diagnosing these cases.

\section{$\underline{\text { LYS029 }}$}

\section{T-cell lymphoma after extranodal marginal zone} lymphoma: coincidence or mimicry?

Shan-Chi $\mathrm{Yu}^{1}$; Sung-Hsin $\mathrm{Kuo}^{2}$; Ming $\mathrm{Yao}^{3}$; Ann-Lii Cheng $^{2}$; Jau-Yu Lia ${ }^{4}$; Cheng-Hsiang Hsiao ${ }^{4}$; Huang-Chun Lien $^{4}$; Chia-Yu Chu ${ }^{5}$; Chung-Wu Li4 ${ }^{5}$

(1) Department of Pathology, National Taiwan University Hospital, Taiwan (2) Department of Oncology, Natonal Taiwan University Hospital (3) Department of Internal Medicine, National Taiwan University Hospital (4) Department of Pathology, National Taiwan University Hospital HsinChu Branch (5) Department of Dermatology, National Taiwan University Hospital

Background: There is no increase in risk of second cancer in extranodal marginal zone lymphoma (EMZL), but second cancers may develop in up to $10 \sim 20 \%$ of EMZL patients. Most second cancers after EMZL are solid tumors. T-cell lymphoma after EMZL is exceedingly rare.

Aim of the study: We report two T-cell lymphomas after diagnosis of EMZL.

Results: Case 1, a 73-year-old woman presented with an ulcerative gastric mass. A diagnosis of stage III gastric EMZL was made, and she was treated with triple therapy for Helicobacter eradication. Sections of the endoscopic biopsy showed infiltration of small lymphocytes, some of which contained pale cytoplasm. The infiltrate was composed of mixed CD $3+T$ cells and CD20 + B cells. There are lymphoepithelial lesions, but the infiltrating lymphocytes in the glands are mainly CD $3+\mathrm{T}$ cells. In situ hybridization 
revealed no light chain restriction. Scattered small cells were positive for EBER. She had sore throat 2 months after initial presentation. Biopsy of the tonsil showed diffuse infiltration of large atypical lymphocytes, which were $\mathrm{CD} 3+/ \mathrm{CD} 4+/$ CD5+/CD56-/CD8-. Scattered cells were EBER+. She was treated with CHOP chemotherapy, but died 6 months after initial presentation. Review of the case, this could be a T-cell lymphoma mimicking EMZL in the gastric biopsy.

Case 2, a 53-year old woman with a history of Hashimoto thyroiditis presented with a bulky thyroid gland. Biopsy showed extensive plasmacytic infiltration, which were mostly kappa+, lambda-. A diagnosis of EMZL with extensive plasmacytic differentiation was made. Bone marrow involvement was noted. Following oral chlorambucil plus steroid and consolidation radiotherapy, the patient achieved a very good partial remission. Exertional dyspnea developed 1 month later, and computed tomography showed ill-defined nodules at bilateral lungs and diffuse lymphadenopathy over the mediastinum and bilateral neck. She was treated with RCHOP chemotherapy, and the lung nodules and lymphadenopathy regressed. Skin rash was also noted, but skin biopsy showed perivascular and interstitial infiltration of large atypical lymphocytes, which were CD3+/CD5-/CD4-/CD8-/ CD30-/CD20-/CD79a-/EBER-.

Conclusions: The two cases highlight the need for a high index of suspicion to exclude mimickers of EMZL, and caution of a second lymphoma in patients with EMZL.

\section{LYS030}

\section{Adult T -cell Leikemia/Lymphoma ( ATLL) hot spot in South India}

Rekha Nair; Priya Mary Jacob; Anila K. R.; Sreejith Nair; Shruti Prem

\section{Regional Cancer Centre Trivandrum}

Background: This study comes from the state of Kerala, South India. Around 600 cases of lymphomas (nodal and extranodal) are analyzed and treated every year in Regional Cancer Centre, Trivandrum. Peripheral T cell lymphomas account for $13.4 \%$ of mature lymphomas (biopsy tissue based data). Anaplastic large cell lymphoma is the commonest $(39.2 \%)$ followed by AITL (15.8\%), PTL NOS (19.6\%)and ATLL (11.4\%).

Aim of the study: Analysis of Chronic lymphoproliferative disorders (CLPD) from June 2009 to February 2012 by flow cytometry imunophenotyping.

Materials and methods: 148 cases of CLPDs were analysed for period of 3 years from June 2009 to February 2012 by flow cytometric immunotyping. A 4color FACSCalibur machine was used. Peripheral blood or bone marrow samples were collected and subjected to stain-lyse-wash technique. Panel of antibodies used included CD20 APC, CD10FITC, FMC7FITC, CD34 PE, CD45PerCP, CD5PE, CD19PerCP, CD23APC, CD3APC, CD7FITC, CD25APC, CD103FITC.

Results: 130 cases $(86.2 \%)$ of B CLPDS and 18 cases (13.8 \%) of T CLPDs were diagnosed. Among the TCLPDs, Adult T cell leukemia/lymphoma (ATLL) was the commonest (50\%), followed by Peripheral T cell lymphoma NOS $(33.3 \%)$ and Sezary syndrome (16.7 \%). All ATLL cases were proved by positivity for serum HTLV-I antibodies. Majority of ATLL cases showed generalized ulcerating erythematous cutaneous nodules. 7 out of 9 ATLL cases showed loss of CD7 but 2 cases showed loss of CD5 only. Conclusions: This study shows a very high occurrence of ATLL cases from the Indian subcontinent which has never been reported before. We are also reporting for the first time (not reported in published literature) the loss of CD5 instead of CD7 in circulating ATLL cells.

\section{LYS031}

\section{T-cell prolymphocytic leukemia with indolent behavior}

Angela M. B. Collie; Claudiu V. Cotta

Cleveland Clinic, Cleveland, OH USA

Background: T-cell prolymphocytic leukemia (T-PLL) is an aggressive mature T-cell neoplasm that generally presents with lymphadenopathy and hepatosplenomegaly in addition to leukemic involvement. Median survival with conventional chemotherapy is approximately 1 year (Matutes E. et al. Blood. 78(12): 3269, 1991.; Dearden CE. Clin Lymphoma Myeloma. 9: S239, 2009.) although this has been improved with chemoimmunotherapy with anti-CD52 monoclonal antibody and stem cell transplantation. Indolent cases of T-PLL have been reported (Garand R. Br J Haematol. 103(2):488, 1998.; Kameoka J. Int J Hematol. 2012.) but they are thought to be rare and most of them progress to an aggressive phase.

Aim of the study: To investigate the clinical outcome of TPLL in our institution.

Materials and methods: The clinical presentation, morphologic, immunophenotypic and cytogenetic features (when available) of 3 random cases from our archives were documented. After the diagnosis was confirmed, the treatment received and the clinical outcome were investigated.

Results: 3 cases of T-PLL had follow-ups between 36 and 39 months from the initial presentation. Two patients presented with skin lesions while the third was incidentally diagnosed with lymphocytosis.

WBC on presentation ranged from 21.8 to $75.9 \mathrm{k} / \mathrm{uL}$, with an absolute lymphocyte count from 16 to $67 \mathrm{k} / \mathrm{ul}$. Peripheral blood 
smears showed atypical lymphocytes with cytoplasmic blebs and nuclear knobs. Flow cytometry in all three cases demonstrated abnormal T-cell populations representing 45 to $95 \%$ of lymphocytes. In one case these lymphocytes were positive for $\mathrm{CD} 4$ and $\mathrm{CD}$, and negative for CD2. In two cases the lymphocytes were positive for $\mathrm{CD} 4$ only, with loss of $\mathrm{CD} 5$ in one case. All three patients demonstrated significant bone marrow involvement. Two cases were positive for TCL1A (one case weakly); the CD4, CD8 positive case was negative for TCL1A. Two cases had normal conventional cytogenetics, one was not investigated. Fluorescence in-situ hybridization studies performed on the case strongly positive for TCL1A showed monosomy 11 (or loss of ATM) and trisomy 17 or gain of TP53.

Each patient received a different treatment. The patient with CD4, CD8 positive cells was treated with photopheresis and bexarotene and was considered to be in remission 39 months after diagnosis, although the bone marrow status was not investigated. A second patient was treated with alemtuzumab 11 months after diagnosis and had mildly increasing lymphadenopathy 36 months after diagnosis. The third patient was treated with combination chemotherapy and had increasing lymphocyte count 39 months after first diagnosis.

Conclusions: These 3 randomly selected cases of T-PLL had a significantly better outcome than what the literature suggests with all the patients alive 36 to 39 months after the diagnosis. It is possible that one of the cases represents a different entity, with an immunophenotype that appears to be identical to that of T-PLL, but the diagnosis is firmly established in the two other cases. This study suggests the possibility that cases of TPLL are currently diagnosed earlier or are more efficiently treated than what the literature reports. New clinical studies to investigate the prognosis of T-PLL are probably necessary in order to make the best therapeutic decisions.

\section{LYS032}

\section{Peripheral T-cell lymphoma metastatic to the eye; a case report}

Veronika Kloboves Prevodnik; Novaković Srdjan; Brozič Andreja; Vidovič Valentinčič Nataša; Novak Andrejčič Katrina; Globočnik Petrovič Mojca

\section{Institute of Oncology, Ljubljana Slovenia}

Background: Intraocular T-cell lymphomas are uncommon. The gold standard for diagnosing intraocular lymphomas is cytopathologic examination of the ocular specimens. Since lymphoma cells in these specimens could be few and mixed with reactive cells the accurate diagnosis could be obtained considering morphological, immunophenotypic and molecular features of lymphoma cells. However, the application of immunophenotypic and molecular techniques in poorly cellular specimens could be limited and the interpretation of the results difficult. Herein we report a case of metastatic intraocular peripheral T-cell lymphoma where the diagnosis of lymphoma was delayed due to the misinterpretation of morphological features and molecular results.

Case study: In a 65-years old woman peripheral T-cell lymphoma was diagnosed by histological examination of left cervical lymph node. Due to poor response to treatment the patients was treated with different modalities of multi-agent chemotherapy. Eighteen months after beginning of treatment while receiving Purinol and Methotrexate, uveitis and vitritis were suspected. Consequently diagnostic vitrectomy was performed. Cytological examination showed poorly cellular sample where small, mature lymphocytes predominated. Some atypical lymphocytes, histiocytes, neutrophils, erythrocytes and naked nuclei were also observed. Atypical lymphocytes were small to medium sized with pale eosinophilic cytoplasm, irregular nuclei with granular chromatin and inconspicuous nucleoli. Regarding to the low number of cells in the vitreous biopsy only PCR based clonality analysis was performed, which showed monoclonal T-cells and polyclonal B-cells. Despite the fact that monoclonal T-cells were demonstrated the case was signed out as negative for lymphoma. We presumed that monoclonality of T-cells was the consequence of CMV infection which was diagnosed at that time. Furthermore, morphological features were nonspecific. One year later the disease progressed and PCR based clonality analysis was performed in FNA lymph node sample. Monoclonal TCR- $\gamma$ rearrangement was detected with a band of similar size compared to the one seen in previous vitreous biopsy. This finding confirmed that monoclonal T-cells in vitreous biopsy were lymphoma cells and not reactive monoclonal T-cells as we thought previously. Conclusions: Metastatic T-cell lymphoma in our case masqueraded as an intraocular inflammatory disorder clinically and cytologically. Methotrexate systemic therapy with its cytolytic effect on lymphoma cells was most probably responsible for the poor cellularity of vitreous specimen and nonspecific cytological findings. Therefore T-cell clonality analysis was crucial for diagnosing metastatic intraocular Tcell lymphoma. Since the data from the literature show the presence of monoclonal T-cells also in reactive lesions it is necessary to compare clonality results of intraocular and lymph node tumor specimens to prevent misinterpretation of these results. When both tumors show similar clonally features diagnosis of lymphoma is favored.

\section{LYS033}

\section{Post Transplant Lymphoproliferative Disorders of T-cell origin: unicenter series of 9 cases}

Tousseyn Thomas ${ }^{1}$; An Herreman ${ }^{2}$; Julie Morscio ${ }^{2}$; Jordi Camps $^{1}$; Emilie Bittoun ${ }^{1}$; Daan Dierickx ${ }^{3}$; Gregor Verhoef ${ }^{1}$; 
Iwona Wlodarska ${ }^{4}$; Christiane De Wolf-Peeters ${ }^{2}$; Xavier Sagaert ${ }^{1}$

(1) University Hospitals KU Leuven (2) Translational Cell and Tissue Research, KU Leuven (3) UZ Leuven (4) Center for Human Genetics, KU Leuven, Leuven, Belgium

Background: Posttransplant lymphoproliferative disorder (PTLD) is a major and life-threatening complication of immunosuppressive drug therapy after solid organ- or bone marrow transplantation, with an overall incidence ranging from $2 \%$ to $10 \%$. PTLD represents a spectrum of lymphoid proliferations. According to the 2008 WHO classification four major categories can be distinguished: early lesion, polymorphic PTLD, monomorphic PTLD and Hodgkin (-like) PTLD. The majority of monomorphic PTLDs are of B-cell origin, and the majority of these cases are EBVrelated. T-cell PTLD was first decribed in 1987, and since then less than 150 cases have been reported, representing only $7 \%$ to $15 \%$ of all PTLDs. T-cell PTLD occur in patients of all ages and the most frequent reported cases include peripheral T-cell lymphoma, not otherwise specified (PTCL, NOS), hepatosplenic T-cell lymphoma (HSTCL) and anaplastic large cell lymphoma (ALCL). In contrast to B-cell PTLD, T-cell PTLDs (with the exception of NK/T cell type) are less frequently EBV-related. T-cell PTLDs tend to occur much later after transplantation than many other PTLDs. About $70 \%$ of these patients developed Tcell PTLD after kidney transplantation and like other PTLDs, T-cell PTLDs are usually extranodal. T-cell PTLD usually does not respond to a reduction of immunosuppression, and has a more aggressive clinical course and a worse prognosis (6 months) compared to the B-cell lesions.

Aim of the study: We retrospectively reviewed all T-cell PTLDs that were submitted to the department of Pathology at the University Hospitals UZ KU Leuven (Belgium) with the purpose to define the incidence and clinicopathological features.

Materials and methods: In the series of 231 consecutive PTLD biopsies (from 174 patients), 15 (from 9 patients) were diagnosed as T-cell lymphoma. Clinical information was retrieved for all cases and morphology, immunohistochemical studies and EBER in situ hybridizations were reviewed.

Results: The incidence of T-cell PTLDs at our center was $5 \%$. Patient's age ranged from 13 to 83 years with a median age of 65 years (mean 52 years). The male to female ratio was $8: 1$, compared to a male predominance of $3: 1$ in the overall PTLD study population. Incidence of T-PTLD was highest after kidney transplantation (4/9) followed by liver(2/9), heart- (2/9) and lung (1/9) transplantation. The organspecific incidences were $0.11 \%, 0.23 \%, 0.39 \%, 0.17 \%$ respectively. No T-cell PTLD occurred after 1092 hematopoietic stem cell transplantations, in contrast to $6 \%$ of the previously reported T-PTLDs. ALK-negative Anaplastic large cell lymphoma (ALCL) was diagnosed in $4 / 9$, peripheral T-cell lymphoma, not otherwise specified (PTCL NOS) in 3/9 and 2/9 were hepatosplenic T-cell lymphoma, the latter representing the two cases with a liver allograft. None of the nine T-cell PTLDs were EBV-related. Extranodal involvement was present in $89 \%$ cases, lymph node involvement was observed in $44 \%$ of cases. The more common extranodal sites included liver (3/9) and skin (3/ 9 ). Bone marrow involvement at time of diagnosis was present in $44 \%$ of cases (4/9). The median time from transplantation to diagnosis of the T-cell lymphoma was 83 months, ranging from 23 to 216 months. 8 patients passed away, with a median overall survival of 4 months. In May 2012, only 1 patient was still alive 160 months following initial diagnosis of primary bone ALCL.

Conclusions: T-cell PTLD cases are rare and, including this series, only 156 cases have been published to date. Therefore data on clinicopathological aspects of the different Tcell PTLD subtypes remain scarce. We encourage others to report their series, to allow further clinicopathological categorization, which is important for prognostic and therapeutic purposes.

\section{LYS034}

\section{Peripheral T cell lymphoma (PTCL)- not otherwise specified (NOS)- Is it truly NOS?}

Tanuja Shet; Bharti Ramnani; Sridhar Epari; Suhas Dhende; Kanchan Kantekure; Manju Sengar; Reena Nair; Hari Menon

Tata Memorial Hospital, Mumbai, India

Background: The peripheral $\mathrm{T}$ cell lymphomas (PTCL) NOS category needs better a diagnostic definition as it is a mixed bag of tumors which are treated similarly but behave heterogeneously. This ongoing study attempts to immunophenotypically analyze 115 PTCL including angioimmunoblastic T cell lymphomas (AITL) to analyze if there are indeed subtypes within the PTCL NOS category. The results of 42 PTCLNOS and 11 AITL are being presented.

Methods: Immunohistochemistry was performed on archival material using the avidin biotin complex method. As these tumors were polymorphous a positive staining required expression in $>75 \%$ of atypical cells.

Results: All tumors expressed CD3. These 53 tumors were classified as CD4 and PD1 coexpressing tumors $(n=9)$, classic AITL $(n=6), \mathrm{CD} 8$ and cytotoxic granule protein (CGP) coexpressing tumors ( $n=9)$, mixed type with expression of two or more lineages $(n=4), 5$ AITL with strong 
expression of CGP, CD 4 and CGP coexpressing tumors( $n=$ 5) and NOS category expressing CGP strongly. Lastly two blastic tumors expressed no markers except CD3. Though CD4 expressing and CGP expressing tumors had propensity for lung and skin involvement this was not statistically significant. Amongst the CD4 expressing tumors one could be retyped as follicular T cell lymphoma. Within CGP, TIA1 expression was commonly seen while granzyme B was only focally present.

A Kaplan Meier analysis revealed that the above classification did not impact overall survival (OAS). However CD4 expressing tumors (log rank value -0.048 ) had better survival while complete loss of CD7 (log rank value - 0.001) and MIB1 $>60 \%$ correlated adversely with OAS. EBER expression in neoplastic cells was seen only in 5 cases. None of these factors impacted the disease free survival. Amongst clinical features $(n=115)$ age $>60$ years, high risk International prognostic index(IPI) and high Ann Arbor stage adversely impacted OAS.

Conclusions: Though PTCLNOS are polymorphous, some immunophenotypic patterns did emerge. Established prognostic parameters including age, IPI and stage also correlated with survival in present analysis.

\section{LYS035}

\section{Multidisciplinary aproach to the diagnosis of deep seated T-cell lymphoma causing jaundice}

Tajana Stoos-Veic ${ }^{1}$; Mario Tadic $^{2}$; Gorana Aralica ${ }^{3}$; Cedna Tomasovic-Loncaric ${ }^{3}$; Vlatko Pejsa ${ }^{2}$; Mario Pirsic ${ }^{2}$; Rajko Kusec $^{2}$

(1) University Hospital Dubrava, Zagreb, Croatia (2) Department of Internal Medicine University Hospital Dubrava

(3) Department of pathology University Hospital Dubrava, Zagreb, Croatia

Background: Peripheral T-cell lymphoma is heterogenous group of mature T lymphoma which is not specifically defined according to WHO 2008 classification. They usually present with peripheral lymph node enlargement and any body site may be affected. We present a case of peripheral T-cell lymphoma, NOS presenting with jaundice and fever and diagnosed by endoscopic ultrasound guided fine needle aspiration cytology (EUS-FNA) and adjuvant molecular methods.

Case report: A 59-year old patient presented in ER with fever, jaundice and dyspnea. He was a diabetic on oral treatment and reported chronic cough and weight loss of $20 \mathrm{k}$ in 2 months. Among other findings, MSCT revealed enlarged mediastinal lymph nodes without pulmonary primary and also dilated intra- and extrahepatic biliary ducts, with stenosis of common bile duct without other cause of obstruction. Biliary brush cytology during ERCP was negative, but EUS-FNA of mediastinal and peripancreatic lymph nodes revealed atipical lymphoid cells and eosinophils. Suspicion of non-Hodgkin lymhoma, possibly of Tcell origin was raised. Since the fresh tissue was not taken at the time of EUS-FNA procedure for clonality testing, DNA was isolated from cytological slides. Multiplex-PCR for TCRgamma with single band in high resolution gel electrophoresis confirmed the monoclonal nature of $\mathrm{T}$ lymphocytes. The patient refused therapy and returned 4 months later with generalized peripheral lymphadenopathy. Submandibular lymph node was removed. Patohistological diagnosis was peripheral T-cell lymphoma, NOS (CD 3, CD5, CD 4 positive, CD2,CD7,CD43 weekly positive, CD8 negative, some of the larger cells CD30 positive, Ki67 was $50 \%$ ).

Patient responded well to the chemotherapy (complete regression of lymph nodes) but died of cerebrovascular insult during the eight course of chemotherapy.

Conclusions: A multidisciplinary approach combining various diagnostic methods (EUS-FNA and molecular testing for clonality) enabled an early diagnosis of deeply seated Tcell lymphoma.

\section{LYS036}

New potential therapeutic strategies in peripheral T-cell lymphomas, the simultaneous inhibition of PI3K and MEK

Esperanza Martín-Sánche ${ }^{1}$; Margarita Sánchez-Beato ${ }^{1}$; Socorro M. Rodríguez-Pinilla ${ }^{2}$; Miguel A Piris ${ }^{3}$

(1) Spanish National Cancer Research Center (CNIO), Molecular Pathology Programme (2) Fundacion Jimenez Diaz, Madrid (3) Hospital Marques de Valdecilla, Santander, Spain

Background: Peripheral T cell lymphomas (PTCL) are a heterogeneous group of very aggressive hematological malignancies that currently lack efficient targeted therapies.

Aim of the study: The very poor prognosis in these patients needs to be improved by new rationally selected therapeutic strategies.

Results: Our genomic studies through gene expression profiling in a series of PTCL cases revealed that PI3K signaling could be an attractive target in PTCL. We observed that (1) PI3K inhibitors (PI3Ki) were the most significant drugs that potentially could reverse our PTCL molecular signature; (2) PIK3CD, encoding the PI3K $\delta$ isoform, was the only one to be significantly correlated with survival pathways in the signature, such as T-cell receptor, NF-kB and CD40 signaling; and (3) PIK3CD was overexpressed in PTCL cell lines 
and primary samples. However, neither genetic nor specific pharmacological PI3K $\delta$ inhibition affected PTCL survival. On the contrary, the pharmacological inhibition of all PI3K isoforms promoted a cell cycle arrest at G1 phase in all PTCL cell lines and induced apoptosis in a subset of them. GSK3 $\beta$ and p70S6K phosphorylation was found as a potential PI3Ki response biomarker. Moreover, we found an increase in pERK levels upon PI3Ki treatment, and interestingly, a high synergistic effect was found between PI3Ki and a MEK inhibitor.

Conclusions: Targeted therapy combining PI3K and MEK inhibition could be an effective therapeutic approach in PTCL.

\section{LYS037}

\section{Spectrum of T-cell lymphomas diagnosed in splenectomy specimens.}

Socorro Maria Rodríguez-Pinilla ${ }^{1}$; Teresa Nam² ${ }^{2}$ Maria del Carmen Toledo ${ }^{3}$; Felipe Casado ${ }^{3}$; Santiago MontesMoreno ${ }^{4}$ Lidia Sánchez ${ }^{5}$ Miguel Ángel Piris ${ }^{6}$; Manuela Mollejo $^{7}$

(1) Pathology Department, FJD (2) Pathology Department, Hospital Genaral de Albacete (3) Haematology Department, Hospital Virgen de la Salud de Toledo. (4) Pathology Department, Hospital Marques de Valdecilla (5) Bithecnonlogy Programme, Immunohistochemistry Unit, CNIO (6) Pathology Department, Hospital Marques de Valdecilla, IFIMAV. (7) Pathology Department, Hospital Virgen de la Salud de Toledo.

Background: Diagnosis of T-cell lymphoma in the spleen is hampered by the rarity of this situation and the lack of clear criteria for recognizing these diseases, with the only exception of hepatoesplenic TCL.

Material and methods: We have reviewed the cases of Tcell lymphoma diagnosed in splenectomy specimens, received as consultation to the CNIO Pathology Department.

Results: The review of the cases showed: four hepatosplenic T-cell lymphomas, TCR gamma positive, two NK/ T-cell lymphomas EBER+CD56+, three peripheral-T-celllymphomas (PTCLs) EBER+ in the setting of familiallymphohistiocytosis, five systemic-PTCLs, AITL and PTCL-NOS, one T-cell prolymphocytic leukemia, three large granular leukemia (LGL) with bone marrow infiltration, three cases of clonal-T-cell-lymphocytosis, with features consistent with LGL, in absence of bone marrow or peripheral blood infiltration, eight cases of primary-splenicPTCLs (PS-PTCLs), with features consistent with LGLs, in absence of bone marrow or peripheral blood infiltration. We found that LGL, clonal T-cell lymphocytosis and primary splenic PTCLs have a similar clinical presentation, morphology and immunophenotype (CD3, TCRBF1, cytotoxic markers and CD57 positivity, with loss of CD5, CD7, BCL2, CD56 and CD8-positivity or double CD4/CD8-negativity), with the only difference of the variable extent of the red pulp infiltration.

Conclusions: Additionally to the already recognized disorders, we have identified a spectrum of lymphoproliferative disorders, presumably LGL-related, characterized by the red pulp infiltration by medium-sized T-cells, in cases lacking bone marrow or peripheral blood infiltration.

\section{LYS038}

The distribution of peripheral T-cell lymphoma in the context of HIV infection differs from that seen in non-immunocompromised patients: a retrospective study of 32 cases

Christiane Copie-Bergman ${ }^{1}$; Véronique Meignin ${ }^{2}$; Laurent Gilardin $^{3}$; Gerard ${ }^{3}$; Eric Oksenhendler ${ }^{3}$; Pierre Sujobert ${ }^{4}$; Bettina Fabiani ${ }^{5}$; Josette Briere ${ }^{2}$; Philipe Gaulard ${ }^{6}$

(1) Département de Pathologie, Inserm U955 (2) AP-HP, Hôpital Saint-Louis, Department of Pathology, (3) Département d'Immunopathologie Clinique, Hôpital Saint-Louis, Université Paris Diderot, (4) Service d'hématologie, hôpital Cochin, APHP (5) Service d'Anatomie et de Cytologie Pathologiques, Hôpital Saint Antoine (6) AP-HP, Groupe Henri Mondor-Albert Chenevier, Département de Pathologie

Background: Lymphomas arising in HIV-infected patients are predominantly aggressive B-cell lymphomas. Peripheral T-cell lymphomas (PTCL) are unusual in this context and their clinical and pathological features are not well known.

Aim of the study: To evaluate the clinicopathologic features of PTCL in HIV-infected patients.

Materials and methods: Thirty-six cases initially diagnosed as PTCL in HIV-infected patients from 1996 to 2012 were reviewed after extensive immunohistochemical and molecular studies and classified according to the WHO classification.

Results: Four cases reclassified as HHV8+ solid primary effusion lymphoma (PEL) with aberrant expression of CD3 were excluded. Among the remaining 32 confirmed PTCLs (26 male, 6 female; mean age, 44 years), the disease presentation was nodal $(n=16)$, or extranodal [incl. bone marrow $(n=3)$, spleen $(n=2)$, skin, lung, eye, breast, gastrointestinal tract, nasal cavity, bone, soft tissues] ( $n=$ 16). They were classified as PTCL-not otherwhise specified (PTCL-NOS, $n=16$ ), NK/T-cell lymphomas, nasal-type (NKTCL, $n=4)$, ALK-negative anaplastic large cell lymphomas (ALK- ALCL, $n=5$ ), hepatosplenic T-cell 
lymphomas (HSTL, $n=2$ ), aggressive NK/T cell leukemia/ lymphoma involving bone marrow and liver $(n=1)$ and Adult T leukemia/lymphoma (HTLV1+, $n=4$ ) whereas angioimmunoblastic T-cell lymphoma (AITL) and ALKpositive ALCL were not seen. Most PTCL-NOS (10/16= $62 \%$ ) showed an activated cytotoxic phenotype (Granzyme $\mathrm{B}+$ ), but were CD56-negative with a variable percentage of EBV-positive tumour cells (6/16). The two HSTL were of a/ $\beta$ and $\gamma / \delta$ origin, respectively. The 5 NKTCL (all EBV+ and cytotoxic) included 1 extranasal nasal-type (involving skin and lung) and 3 unusual cases with nodal involvement. Conclusions: The distribution of PTCLs in HIV-infected patients differs from that seen in non immunocompromised patients with absence of AITL and ALK-positive ALCL, common derivation from cytotoxic lymphocytes $(22 / 32$, $69 \%$ ), variable association with EBV and frequent presentation as extranodal disease. In this setting, staining for HHV8 is required to exclude PEL with aberrant expression of T-cell antigens.

\section{LYS039}

\section{Distinctive role of microRNA expression profiles on T-cell lymphomas}

Yasemin Sahin ${ }^{1}$; Gulsah Kaygusuz ${ }^{2}$; Hilal $\mathrm{Ozdag}^{3}$; H. Gokhan $\mathrm{Ilk}^{3}$; Isinsu $\mathrm{Kuzu}^{2}$

\section{(1) Ankara University Department of Pathology (2) SPMD} (3) $\mathrm{PhD}$

Background: Comparing to B cell lymphomas, success in the therapy of mature T cell lymphomas (TCLs) is lower due to the limited knowledge on their pathogenesis and differential diagnosis. MicroRNAs (miRNAs) are small noncoding evolutionally preserved RNA molecules regulating posttranscriptional gene expression. They have effects on several cellular biological functions such as proliferation, differentiation, apoptosis, hematopoiesis and immune regulation. Although the miRNA expression profiles show variations in different neoplasia, there is limited information about their role on pathogenesis of TCLs.

Aim of the study: To compare the miRNA expression profiles of peripheral T-cell lymphoma, NOS (PTCL, NOS) and angioimmunoblastic T-cell lymphoma (AILT).

Materials and methods: The disease group contains six AILT and five PTCL, NOS cases whereas the control group consists of three cases of benign reactive hyperplasia. Total RNAs (100 ng) extracted from formalin fixed paraffin embedded tissues were used for hybridization. Human miRNA microarray release 14.0 platform (Agilent Tech) was performed for miRNA expression analysis. GeneSpring GX software (Agilent Tech) was used for bioinformatics analysis.
Results: The cases of PTCL, NOS and AILT in the disease group were compared with the reactive control group. More than two-fold change were found in 53 miRNAs ( 28 were upregulated, 35 were downregulated) between disease and control groups. Twenty-seven miRNAs (15 downregulated, 12 upregulated) showed more than five-fold change between the disease and the control groups ( $\mathrm{t}$ test, $p<0.05$ ). Three miRNAs, which have functions in T-cell receptor signaling pathways were identified as discriminative between each of the lymphoma subtypes and also the control group (Oneway ANOVA, Benjamini-Hochberg corrected, $p<0.05$ ).

Conclusions: PTCL, NOS and AILT cases show different expressions of miRNAs related to the T-cell receptor signaling pathways when compared to the control cases. The three miRNAs may be a candidate marker for TCL subtypes and their target pathways will help to understand the biology of these disease entities.

\section{LYS040}

T-cell markers expression in aggressive B-cell lymphomas: pearls or pitfalls - The story of two cases

Tihomir Dikov ${ }^{1}$; Nikolay Stoyanov ${ }^{2}$; Vera Stoeva ${ }^{2}$; Margarita Guenova $^{2}$

\section{(1) Medical University Sofia Bulgaria (2) NSBALHZ}

Background: Aggressive B-cell lymphomas with immunoblastic differentiation and plasmablastic lymphoma notoriously tend to underexpress widely employed pan-B-cell markers, e.g. CD20 \& CD22. Aberrant expression of Tcell markers in this setting may lead the unwary to an erroneous conclusion with potential clinical consequences. Material and methods: We present two cases exemplifying each of the aforementioned categories.

Results: Case1. A 75-year-old male was referred to our institution with a 3 year unfortunate history of frequently relapsing tumor masses, extensively involving facial structures. He was misdiagnosed with nasopharyngeal cancer and respectively treated with platinum-based chemotherapy and local radiotherapy. Upon third relapse, sample tissue, as well as all previous biopsies, were reviewed to reveal identical striking proliferation of highly atypical large cells with unequivocally plasmablastic appearance. On immunophenotyping tumor cells were consistently positive for CD138, MUM-1 and CD56 low while negative for CD19, CD20, CD22, CD45, PAX-5, CD10, TdT, EMA and CK in all samples. Unexpectedly, a bright positive surface expression of CD3 was noted. Ki-67 labeling was about $70 \%$. Insitu hybridization (ISH) techniques revealed only kappa, but no lambda light chain RNA cytoplasmic transcripts. EBVencoded RNA (EBER) positively stained neoplastic nuclei. 
Patient's serum showed no paraprotein and disease-free bone marrow. At that time the patient rapidly deteriorated and succumbed to his disease.

Case2. A 47-year-old female referred for rapidly enlarging cervical, axillary and mediastinal lymph nodes was admitted and subsequently biopsied at our hospital. The lymph node structure was diffusely effaced by proliferation of large to giant cells bearing immunoblastic appearance with prominent inclusion-like nucleoli. Immunohistochemistry disclosed a distinctive aberrant mature B-cell phenotype: CD45+ MUM1+ PAX-5+ CD79a+ with negativity for CD20, CD22, CD10, BCL6, CD138 and CD56. Ki -67 highlighted more than $90 \%$ of the neoplastic nuclei. To complicate matters, aberrant coexpression of CD45RO and CD30 was observed. Importantly, CD3 was negative. EBER and cytoplasmic RNA for kappa light chains stained negative, but lambda showed light chain restriction in the neoplastic population. The patient received a CHOP-therapy but was soon lost to disease complications.

Conclusions: These two cases are similar in representing an arrested stage of post-germinal center B-cell differentiation towards plasma cells. Lack of B-cell markers favors advanced differentiation, but it conveys no favorable clinical outcome. On one hand, T-cell marker expression in B-cell lymphomas may be misleading when arriving at the correct diagnosis, on the other hand the aberrancy confirms the neoplastic nature of the cells. The absence of common B-cell markers renders the patients ineligible for currently available chemoimmunotherapeutic regimens. Alternatively T-cell marker expression could be employed as a therapeutic target.

Acknowledgements: This study was supported by the National Science Fund, Ministry of Education and Science.

\section{LYS041}

\section{Comparative genomic hybridization in peripheral T-cell lymphoma}

Christian Bastard $^{1}$; Sylvain Mareschal ${ }^{1}$; Virginie Fataccioli ${ }^{2}$; Jean Soulier ${ }^{2}$; Philippe Gaulard ${ }^{2}$

(1) Centre Henri Becquerel, Rouen (2) Département de pathologie, hopital Henri Mondor

Background: Peripheral T cell lymphomas (PTCL) constitute a heterogeneous group of malignancies developed in counterpart of different normal lymphoid populations. Most frequently cases can be classified as angioimmunoblastic T-cell lymphoma (AITL), derived from follicular helper T-cells (TFH), and PTCL not otherwise specified (PTCL-NOS) the normal counterpart of which is not perfectly identified. A differential diagnosis between these two subgroups can be difficult and both are associated with a poor outcome. The genetic background of these diseases is poorly characterized and a better understanding of their pathophysiological basis will provide clues for better diagnostic and therapeutic approaches.

Materials and methods: The Tenomic study collected more than 600 samples from patients with T-cell lymphoma. All cases were centrally reviewed and well characterized cases benefited from different complementary analyses. Comparative genomic hybridization data were available for more than 200 patients with a diagnosis of AITL or PTCL-NOS. We present the preliminary results obtained in 169 patients. 19 patients were studied using a 400k Agilent array, 150 were studied using a $4 \times 180 \mathrm{k}$ Agilent array, according to the manufacturer recommendation (Pr Jean Soulier). Data were analysed using various available programs and the DNA analytics program (Agilent).

Results: The interpretation of CGH data in PTCL is difficult, as the ratio between normal and tumoral cells can vary from about 20 to $90 \%$. As compared to results obtained in other lymphoma types, mainly of B-cell lineage, the number of recurring commonly deleted or amplified regions appears very low. The most convincing data concerns the CDKN2A-CDKN2B region, deleted in $13 \%$ of cases (monoallelic+biallelic deletions) or two deleted regions on chromosome 5, involving the MYO10 and DMXL1 genes. Amplifications mainly involve chromosome 7 and the CDK5-containing region.

Conclusions: Both deleted and amplified regions are under further investigation, in order to identify other associated mechanisms, which could be directly involved in lymphoma pathogenesis.

\section{LYS042}

Clinicopathologic features of HTLV-1 associated adult T-cell lymphoma/leukemia outside of Japan

April Chiu ${ }^{1}$; Matthew Lunning ${ }^{1}$; Melissa Pulitzer ${ }^{1}$; Oscar Lin ${ }^{1}$; Jocelyn Maragulia ${ }^{1}$; Alison Moskowitz ${ }^{1}$; Patricia Myskowski ${ }^{1}$; Andrew Zelenetz ${ }^{1}$; Steven Horwitz ${ }^{1}$; Julie Teruya-Feldstein ${ }^{1}$

(1) Memorial Sloan Kettering Cancer Center, New York, USA

Introduction: Adult T-cell lymphoma/leukemia (ATLL) is a mature T-cell malignancy associated with human T-cell lymphotropic virus-1 (HTLV-1) infection, and is associated with poor prognosis despite aggressive treatment approaches. HTLV-1 is endemic to parts of Japan, western South America, the Caribbean islands, and western Africa. HTLV-1 infection and development of ATLL outside of these regions most commonly occur among immigrant populations. The clinical and pathologic features of ATLL have been well characterized in Japanese patients, but to a lesser extent outside of Japan. 
Material and methods: To explore the clinicopathologic features and clinical outcomes of ATLL patients at our institution, we conducted a retrospective review of pathologic data and individual charts of ATLL patients treated from 1998 to 2012 at MSKCC.

Results: We identified 45 patients with ATLL, all of whom had positive HTLV-1 antibodies with a confirmatory Western blot. Patient characteristics were: median age 54 (range 3082); male:female 17:28; Caribbean-34, American-6, Western African-2, Peruvian-2, and Grecian-1. Disease subtype: Acute24, Lymphoma-13, Chronic-5, and Smouldering-3. At presentation, hypercalcemia, circulating tumor cells, and osteolytic lesions were noted in $44 \%, 40 \%$, and $6 \%$ of cases, respectively. Adequate clinical data were present in $62 \%$ of the patients for IPI risk category assignment, $65 \%$ of whom had high-intermediate to high risk IPI (3-5). Biopsy material was available in our file in 13 patients, including lymph node (LN; 7 cases), skin (4 cases), bone marrow (1 case), and gastric (1 case) biopsies. Morphologically the tumor cells were medium to large ( 9 cases), small pleomorphic ( 3 cases), and anaplastic (1 case). The cases were positive for CD2 (7/8), CD3 (12/12), CD5 (8/11), CD4 (12/12), and CD8 (0/12). Partial or complete loss of $\mathrm{CD} 7$ expression was observed in 9/11 cases. In addition, $11 / 11$ cases were positive for $\mathrm{CD} 25$, while $7 / 13$ cases were positive for CD30. An ALK immunostain was performed and negative in 6 of the CD30 positive cases. All 4 cutaneous cases were CD30 negative. Molecular study showed clonal Tcell receptor gene rearrangement in 5/5 tested cases. Cytogenetic data were available in 3 cases, all of which showed a complex karyotype. The median overall survival of all 45 patients was 12.9 months. Hypercalcemia and evidence of circulating disease at presentation was not predictive of overall survival $(p=.225 ; p=.141)$. However, an IPI of 2 was predictive of improved overall survival $(p<0.01 ; p<0.001)$.

Conclusions: HTLV-1 associated ATLL outside of Japan remains an under-characterized malignancy in a frequently underserved patient population. The classical clinical findings of hypercalcemia, circulating disease, and osteolytic bone lesions were seen in a minority of patients in our review and did not affect overall survival. Pathologically, ATLL remains a disease largely recognized only by demonstrating serologic evidence of HTLV-1 infection due to its wide morphologic spectrum. The subtype distribution and expression for $\mathrm{CD} 3, \mathrm{CD} 4$, and $\mathrm{CD} 25$ appears to mirror the Japanese data, although differences include younger median age and female predominance in our series. Of interest, CD30 expression was seen at a higher prevalence compared to Japanese reports of $19 \%$. As such, ATLL should be considered in the differential diagnosis of CD30 positive T-cell lymphoproliferative disorders in non-Japanese populations. As novel therapies targeting CD30 such as SGN-35 have emerged, assessment of CD30 expression may become clinically important in ATLL patients outside of Japan.
LYS043

\section{Aberrant CD20 expression in T-cell lymphomas}

Aysegül Üner ${ }^{1}$; Nalan Akyürek ${ }^{1}$; Arzu Salam Ayhan ${ }^{2}$; Pinar Uyar Göçün ${ }^{1}$

(1) Gazi University, Medical Faculty, Department of Pathology (2) Hacettepe University, Medical Faculty, Department of Pathology

Background: $\mathrm{T}$ cell lymphomas co-expressing B cell markers is extremely rare and may pause a potential pitfall in diagnosis. We report two cases of T-cell lymphoma with aberrant CD20 expression.

Material and methods: Report of two cases with histology and immunophenotype.

Results: The first case was a 36 year-old man who presented with a septal perforation and nasal obstruction. A nasal biopsy showed a diffuse, angiocentric, and angiodestructive infiltrate composed of small and large atypical lymphoid cells with round to slightly irregular nuclei, finely granular chromatin, small nucleoli, and moderate amounts of clear cytoplasm. Focal areas of coagulative necrosis were noted. Immunohistochemically, the tumor cells were positive for CD20, CD2, CD3, CD56, TIA1, perforin and granzyme B. The cells were negative for CD5, CD7, CD4 and CD8. EBER in situ hybridization for EBV was strongly and diffusely positive in the tumor cells. Therefore, a diagnosis of nasal type NK/T cell lymphoma with aberrant expression of CD20 was rendered. The patient was treated with 4 cycles of CHOP (cyclophosphamide, doxorubicin, vincristine, and prednisone) and local radiotherapy. Thereafter autologous stem cell transplantation was performed. He relapsed 2 years later with recurrent disease in the nasal septum and died 30 months later.

The second case was 74 year-old woman who presented with cervical lymphadenopathy. The lymph node biopsy revealed a diffuse infiltration of medium and large atypical lymphoid cells with irregular nuclei. Mitotic figures and apoptotic bodies were abundant. Immunohistochemically, the tumor cells were positive for CD20, CD43, CD3 and $\mathrm{CD} 2$. The cells were negative for CD56, CD79a, ALK, granzyme $\mathrm{B}, \mathrm{CD} 5, \mathrm{CD} 7, \mathrm{CD} 4$ and $\mathrm{CD} 8$. A diagnosis of peripheral T-cell lymphoma, NOS, with aberrant CD20 expression was rendered. The patient was treated with 6 cycles of CHOP and local radiotherapy. The patient relapsed 2 years later and died 46 months later.

Conclusions: We present two cases of T-cell lymphoma with aberrant expression of CD20. CD20 is generally regarded as being specific for B cells, which may cause diagnostic difficulties in T-cell lymphomas. A possibility of treating such patients with Rituxumab should be considered. 


\section{LYS044}

Detection of T-cell clonality in formalin-fixed, paraffin-embedded tissue: a comparative analysis of different protocols

Irina Bonzheim ${ }^{1}$; Florian Fröhlich ${ }^{1}$; Patrick Adam ${ }^{1}$; Sema Colak $^{1}$; Gisela Metzler ${ }^{2}$; Leticia Quintanilla-Martinez ${ }^{1}$; Falko Fend ${ }^{1}$

(1) Institute of Pathology and (2) Department of Dermatology, University Hospital Tübingen, Tübingen, Germany

Background: Peripheral T-cell lymphomas are a morphologically and phenotypically heterogeneous group of nonHodgkin lymphomas (NHL), which frequently require molecular determination of clonality for a definitive diagnosis. A number of PCR-based protocols with increasing complexity in primer design have been published in the last decades for the detection of T-cell receptor (TCR) gene rearrangements. Whereas most protocols target only the less complex $\mathrm{TCR} \gamma$ locus, the multiplex assays developed by the BIOMED-2 consortium additionally include the TCR $\beta$ locus and have shown superior sensitivity for the detection of clonality in T-NHL. However, they have been developed primarily on fresh frozen tissues, and may lead to overinterpretation due to their multiple product sizes ranging up to $350 \mathrm{bp}$.

Materials and methods: To compare the performance of two relatively simple, first-generation TCR $\gamma$ PCR protocols published by McCarthy et al. (Diagn Mol Pathol 1:173-179, 1992) and Trainor et al. (Blood 78:192-196, 1991) with the BIOMED-2 TCR $\gamma$ and TCR $\beta$ assays in formalin-fixed, paraffin-embedded (FFPE) tissues, using fluorescencelabeled primers and GeneScan analysis. All analyses were run at least in duplicate, and results were classified as clonal, polyclonal, equivocal or technically not evaluable by two independent observers.

Results: FFPE samples of 52 peripheral T-NHL, and 55 controls consisting of $20 \mathrm{~B}-\mathrm{NHL}$ and 35 reactive lymphoid tissues, were analysed. Special emphasis was put on "difficult" cases such as skin $(n=21)$ and EDTA-decalcified bone marrow $(n=23)$ biopsies. A 50 -case subset including all samples with false negative or false positive results with the two simple TCR $\gamma$ protocols was evaluated additionally with the BIOMED-2 assays. Four out of six, previously false negative T-NHL were positive with the BIOMED-2 TCR $\beta$ protocol, increasing sensitivity for this selected subgroup to $92 \%$, as compared to $64 \%$ (McCarthy) and $72 \%$ (Trainor). The overall specificity $(80 \%)$ achieved with the BIOMED-2 assays was comparable to Trainor $(84 \%)$ and McCarthy (88 \%) protocols, but incomplete TCR $\beta$ DJ rearrangements were identified in 4/10 B-NHL cases.
Conclusions: The combined BIOMED-2 TCR $\gamma$ and TCR $\beta$ assays show superior sensitivity for the detection of T-cell clonality in peripheral T-NHL as compared to simpler TCR $\gamma$ portocols. However, the size ranges to be attained and the complexity of BIOMED-2 protocols require both good DNA quality and experience in interpreting GeneScan patterns.

\section{LYS045}

CD10 and ICOS expression by multiparametric flow cytometry in angioimmunoblastic T-cell lymphoma

Lucile Baseggio ${ }^{1}$; Alexandra Traverse Glehen ${ }^{2}$; Françoise Berger $^{2}$; Martine Ffrench $^{3}$; Dominique Morel $^{3}$; Jean-Pierre Magaud $^{3}$; Gilles Salles ${ }^{1}$; Pascale Felman ${ }^{3}$

(1) CHU Lyon-Sud/HCL (2) Service d'anatomie pathologique (3) Laboratoire d'Hématologie

Background: Angioimmunoblastic T-cell lymphoma (AITL) is immunologically defined by expression of the CD10 and the follicular helper T-cells (TFH) markers such as CXCR5 and ICOS, a profile mainly reported by immunohistochemistry (IHC). Materials and methods: Here using multiparametric flow cytometry (FCM), the relevance of ICOS to AITL diagnosis was evaluated in lymph node (LN, $n=20)$ as well as in peripheral blood (PB, $n=15)$ among a series of 34 AITL (histologically proven), in addition to the CD10 expression (available in $31 \mathrm{LN}$ and $20 \mathrm{~PB}$ specimens).

Results: In this series, CD10 expression was present in 28/31 (90 \%) LN and in 15/20 (75 \%) PB cases and ICOS in 17/19 (90 \%) LN and in 8/13 (53\%) PB cases, whereas neither significant CD10 nor ICOS T-cells were identified in the control group ( $\mathrm{LN}$ with reactive hyperplasia $=10, \mathrm{~PB}$ of healthy donors $=15$ ). Eight 8 pairs ( $\mathrm{LN}$ and $\mathrm{PB}$ ) have been studied at the same time and suggest that the neoplastic T-cells population could be composed of immunologically different cell populations: CD10+/ICOS+, CD10-/ICOS-, CD10-/ICOS+, CD10+/ICOSConclusions: Multiparametric FCM approach allowed us to confirm the frequent blood dissemination in AITL and to show that circulating neoplastic T-cells correspond more often to a CD10 positive subset than to an ICOS positive subset.

\section{LYS046}

CD10 is expressed by a subset of TFH cells: further evidence that its expression in angioimmunoblastic T-cell lymphoma (AITL) reflects ontogeny and is not aberrant

Sylvia Hoeller ${ }^{1}$; Patricia Amé-Thomas ${ }^{2}$; Nadine MartinGarcia $^{3}$; Catherine Artchounin ${ }^{3}$; Christiane CopieBergman $^{3}$; Karin Tarte ${ }^{2}$; Philippe Gaulard ${ }^{4}$ 
(1) Institute of Pathology Basel (2) INSERM U917, Université Rennes 1 (3) Departement of Pathology, Inserm U955 (4) Departement of Pathology, Hopital Henri Mondor, France

Background: CD10 is a well-known marker of germinal center (GC) B-cells. CD10 is also variably expressed by neoplastic $\mathrm{T}$ cells in angioimmunoblastic T-cell lymphoma (AITL), which is known to derive from the follicular helper T cell (TFH) subset located with the GC. However, CD10 yet has not been reported in normal TFH and CD10 expression is regarded as aberrant in AITL.

Aim: To identify CD10-expressing T cells in reactive conditions and B-cell lymphomas and to characterize this population as a subset of TFH.

Material and methods: Reactive lymph nodes $(n=15)$ or tonsils ( $\mathrm{i}=6$ ), 24 follicular lymphoma (FL), 9 marginal zone lymphoma (MZL), 5 mantle cell lymphoma (MCL) and 6 nodular lymphocyte predominant Hodgkin lymphoma (NLPHL) were analyzed for the presence of CD10expressing $\mathrm{T}$ cells by immunohistochemistry.

Results: 17/21 (81\%) reactive lymph nodes or tonsils showed a variable number of strong (stronger than in GCB cells) CD10-positive lymphocytes distributed throughout the GC, with tendency to accumulate at their periphery, paralleling the distribution of PD1+, CXCL13+ and/or ICOS+ cells. Strong CD10+ cells were also observed in FL (11/24, $46 \%$ ) and MZL (9/9, $100 \%$ ), but neither in MCL nor NLPHL. These cells did not co-express PAX5, but stained for CD3, ICOS, PD1 and/or CXCL13. Multicolor flow cytometry confirmed a stable proportion of CD10expressing CD4pos/CXCR5hi/ICOShi TFH cells within reactive tonsils $(19+/-8 \%, n=9)$. Interestingly, the percentage of CD10-positive TFH is also strongly correlated with the percentage of CD19posCD10pos GCB cells.

Conclusions: Expression of CD10 in mature T-cells is physiological and can be attributed to a subset of TFH in reactive GC and in the microenvironment of FLs and MZL. Therefore, CD10 in AITL appears to reflect ontogeny and likely is not aberrant. Cell interactions in reactive and neoplastic GC and in AITL might play a role in the regulation of CD10.

\section{LYS047}

Cutaneous follicular helper T-cell (TFH) lymphomas: a series of 6 cases highlighting new clinicalpathological aspects

Nicolas Ortonne ${ }^{1}$; Fatima Kebir ${ }^{2}$; Denis Chatelain ${ }^{3}$; Guillaume Chaby $^{4}$; Agnes Carlotti ${ }^{5}$; Marie-Hélène Lecaudey-Hansen ${ }^{6}$; Francois $\mathrm{Comoz}^{7}$; Philippe Gaulard ${ }^{2}$

(1) France (2) Département de pathologie, hopital Henri Mondor (3) Service d'anatomie et cytologie pathologiques, CHU
d'Amiens (4) Service de dermatologie, CHU d'Amiens (5) Departement de Pathologie, Hopital Henri Mondor, Creteil, France (6) Cabinet d'anatomie pathologique (7) Service d'anatomie et cytologie pathologiques, centre hospitalier de Caen

Background: Some TFH markers are expressed in Sézary syndrome, primary cutaneous CD4+ pleomorphic small medium sized T-cell lymphomas, and secondary cutaneous lesions of angioimmunoblastic T-cell lymphomas. There is however a proportion of cutaneous T-cell lymphomas that do not fit into these categories, that may be related to the previously described nodal peripheral T-cell lymphomas (PTCL), NOS follicular variants, and recently considered to represent a distinct sub-group among primary cutaneous T-cell lymphomas and termed primary cutaneous TFH lymphomas (PCTFHL), through two small series of 2 and 5 cases.

Aim of the study: to report new clinical and pathological aspects of PCTFHL.

Materials and methods: In the past 4 years, we have identified such lymphomas in 14 patients and reviewed 6 cases with complete information. We conducted a retrospective study recording clinical features and morphological, phenotypical (CD2, CD3, CD4, CD5, CD7, CD8, CD10, CXCL13, PD1, ICOS) and molecular (EBV in situ hybridization and B and T-cell clonality studies) parameters.

Results: The patients had multiple nodules $(n=4)$, an ulcerated tumor $(n=1)$ or an eruption $(n=1)$. One had a regional enlarged lymph node, showing partial involvement by a PTCL, NOS, another a polyadenopathy and 2 a leukemic component. Epidermotropic neoplastic TFH cells were evident in 2 cases, and in one the neoplastic cells were large and expressed CD30. T-cell antigen loss was found in 3 cases (CD2 or CD5). The architecture of the infiltrates varied from a perivascular infiltrate with monocytoid clear cells $(n=2)$ to a diffuse dermal/subcutaneous $(n=3)$ or a nodular infiltrate with follicular dendritic cells hyperplasia $(n=1)$. The neoplastic T-cells were $\mathrm{CD} 4+$ with variable expression CXCL13, PD1, ICOS and BCL6 ( $<5 \%$ to over $50 \%$ ), while CD10+ lymphocytes were rare $(n=2)$. Plasma cells were present in 3 cases, with a monotypic kappa light chain expression in one. Three had an EBV+B-cell population, giving rise to an associated diffuse large B-cell lymphoma in two (skin and lymph node). All had a dominant cutaneous T-cell clone.

Conclusions: PCTFHL have a broad spectrum of clinicalpathological presentation. They have to be distinguished from the other cutaneous T-cell lymphomas that are known to express some TFH markers. Some are epidermotropic and they may give rise to an $\mathrm{EBV}+\mathrm{DLBCL}$. 


\section{LYS048}

Use of a new, highly sensitive anti-PD-1 (CD279) antibody, EH33, as part of a panel of follicular helper $T$ cell (TFH) immunophenotypic markers to distinguish TFH-derived neoplasms from other $T$ cell neoplasms

Olga Pozdnyakova ${ }^{1}$; Gordon J. Freeman ${ }^{2}$; David D. Dorfman ${ }^{1}$ (1) Brigham and Women's Hospital (2) Department of Medical Oncology, Dana Farber Institute

Background: In 2006, we identified the first anti-PD-1 antibody, EH12, as an immunophenotypic marker of TFH cells and angioimmunoblastic lymphoma (AIL; Dorfman et al. Am J Surg Pathol 2006; 30:802-810). Here we employ EH33, a new, highly sensitive mouse monoclonal antibody for PD-1, as part of a panel of TFH immunophenotypic markers for the characterization of TFH-derived neoplasms and differentiation from other $\mathrm{T}$ cell neoplasms.

Material and methods: The anti-PD-1 antibody EH33 was generated by immunization of mice with recombinant human PD-1 fusion protein, generation of hybridomas, and screening of supernatants for specific western blotting of PD-1 transfected cells. EH33 was then employed for immunohistochemical staining of formalin-fixed paraffinembedded tissue sections from a wide range of T-cell derived neoplasms, and compared to that of other TFH immunophenotypic markers, including CD200, ICOS, and CXCL13.

Results: Most T cell-derived neoplasms, including T lymphoblastic lymphoma/leukemia (10 cases), T-PLL (11 cases), NK/T cell lymphoma (10 cases), ALCL (33 cases; $16 \mathrm{ALK}+$ and 17 ALK-), were negative for all TFH markers, with a minority of cases immunoreactive for one TFH marker. In contrast, angioimmunoblastic lymphoma (33 cases) was immunoreactive for all TFH markers studied in the vast majority of cases, with focal (12 cases) or diffuse (21 cases) staining for PD-1. Cases of possible primary cutaneous CD4+ small/medium $\mathrm{T}$ cell lymphoma were also immunoreactive for all TFH markers studied (6 cases). However, cutaneous T-cell lymphomas classified as mycosis fungoides were typically immunoreactive for PD-1 (15/17 cases) and at least one additional TFH marker in the majority of cases. Only a minority of cases of mycosis fungoides with large cell transformation (13 cases) was immunoreactive for PD-1, and all were uniformly negative for CD200. The majority of cases of PTCL, NOS (34/58 cases; $59 \%$ ) were immunoreactive for one or more TFH markers (PD-1 > CD200 > ICOS). Twenty-four cases were immunoreactive for one TFH-cell marker, 5 cases were immunoreactive for $2 / 3$ TFH markers, and 5 cases were immunoreactive for $3 / 3$ TFH markers; the latter group may represent early, evolving angioimmunoblastic lymphoma or another TFH-derived neoplasm.
Conclusions: Immunoreactivity for PD-1 and other TFH markers identifies at least two unique subsets of $\mathrm{T}$ cell neoplasia and substantiates the model of $\mathrm{T}$ cell neoplasia as a process in which $\mathrm{T}$ cells at specific stages of differentiation/activation undergo neoplastic transformation. A panel of TFH immunophenotypic markers is helpful for the characterization of PD-1+ $\mathrm{T}$ cell neoplasms. The availability of anti-PD-1 immunotherapeutic agents may provide an opportunity to treat specifically TFH-derived neoplasms.

\section{LYS049}

The significance of MUM1/IRF4 protein expression and IRF4 translocation in the differential diagnosis of $\mathrm{CD30}+$ cutaneous $\mathrm{T}$ cell lymphoproliferative disorders: A study of 53 cases

Tugce Esen ${ }^{1}$; Cuyan Demirkesen ${ }^{3}$; Candan Eker ${ }^{2}$; Hakki Kumusoglu ${ }^{2}$; Nukhet Tuzuner ${ }^{2}$

(1) Pathology Department of I.U Cerrahpasa Medical Faculty (2) Medimiks Medical Biotechnology, R\&D Lab, Turkey

Background: With current laboratory techniques and clinical and morphologic information, it is not possible to fully distinguish between $\mathrm{CD} 30+$ lymphoproliferative disorders (CD30+ LPD), transformed mycosis fungoides (T-MF) and systemic ALK- anaplastic large cell lymphoma (ALCL) with skin involvement. In recent studies, IRF4 (Interferon regulatory factor-4) translocation was reported to be useful in the diagnosis of primary cutaneous anaplastic large cell lymphoma.

Aim of the study: The aim of our study was to investigate the utility of IRF4 translocation and expression in skin biopsies.

Materials and methods: Our study group was composed of 26 lymphomatoid papulosis (LyP), 13 primary cutaneous anaplastic large cell lymphoma (PCALCL), 9 T-MF. Three cases of systemic ALK(-)ALCL, 1 systemic ALK(+)ALCL and 1 classic Hodgkin Lymphoma (CHL) were taken as control group. We developed a dual color break apart FISH probe from related BAC clones for the detection of IRF4 rearrangements from tissue samples. We performed FISH and immunohistochemistry for the expression of MUM1/ IRF4.

Results: Forty of 53 (75\%) cases were successfully evaluated with IRF4-FISH method. Six of 8 PCALCL cases had IRF4 translocation while all 32 cases of 19 LyP, 8 T-MF, 1 systemic ALK(-)ALCL, 1 systemic ALK(+)ALCL and 1 CHL had no IRF4 translocation. Twelve of 13 PCALCL (92\%), 16 of $26 \operatorname{LyP}(61 \%), 8$ of 9 T-MF(90\%) and 4 of 5 cases in control group $(80 \%)$ displayed immune reactivity 
with MUM1/IRF4. Among all cases the detection of IRF4 translocation had a specificity and positive predictive value for PCALCL of $90 \%$ and $87,5 \%$ respectively ( $p=0,0001$, Fisher's exact test). In contrast IRF4 protein expression was distributed widely in these cases, without any predictive value. Conclusions: In contrast to MUM1/IRF4 protein expression, IRF4 translocation represents the most specific finding to differentiate PCALCL from LyP, CD30(+) T-MF and ALK(-) systemic ALCL with skin involvement. However, it should be kept in mind that IRF4 translocation must be interpreted together with the clinical, histopathological and immunophenotypical findings.

\section{LYS050}

The role of $\mathrm{C} / \mathrm{EBP} \beta$ in the phenotype of ALK+ anaplastic large cell lymphoma

Janine-Alison Schmidt ${ }^{1}$; Irina Bonzheim ${ }^{2}$; Sabine Schäfer ${ }^{2}$; Falko Fend $^{2}$; Leticia Quintanilla-Martinez ${ }^{2}$

(1) Instiute of Pathology, Tübingen, Germany (2) Institute of Pathology and Neuropathology, University Hospital Tuebingen, Eberhard-Karls-University, Germany

Background: ALK + anaplastic large cell lymphoma is characterized by the loss of pan T-cell antigens and the unusual expression of myelomonocytic surface markers. The forced overexpression of the transcription factor $\mathrm{C} /$ $\mathrm{EBP} \beta$ in $\mathrm{B}$ and T-cells has been shown to induce transdifferentiation into macrophages.

Aim of the study: Since $C / E B P \beta$ has been shown to play a central role in the pathogenesis of ALK+ALCL, the aim of the study was to investigate its influence on the unusual phenotype of ALK+ALCL.

Materials and methods: The expression of 242 surface antigens was investigated in ALK+ALCL cell lines before and after the specific knockdown of C/EBP $\beta$ using the BD Lyoplate Human Cell Surface Marker Screening Panel by flow cytometry. A highly specific C/EBP $\beta$-shRNA was transduced by lentiviral infection. The expression of significant surface markers was validated by immunohistochemistry and Western blot in primary ALK+ and ALK- ALCL cases.

Results: The surface marker screening confirmed the loss of T-cell specific markers, such as TCR chains and CD3, and overexpression of EMA and activation markers CD25 and CD30 but did not support the unusual expression of myeloid markers as $\mathrm{CD} 13$ or $\mathrm{CD} 33$, as previously described. Activation surface markers, including CD25, CD30, CD97 and CD98, showed downregulation after $\mathrm{C} / \mathrm{EBP} \beta$ knockdown indicating a role for $\mathrm{C} / \mathrm{EBP} \beta$ in the activation of ALK +ALCL cells. CD147 (EMMPRIN) was strongly expressed in ALK+ALCL cells, and was downregulated after C/EBP $\beta$ knockdown. Interestingly, CD147 was differentially expressed in ALK+ALCL when compared to ALK- ALCL primary cases.

Conclusion: Surface marker screening in ALK+ALCL revealed an influence of $\mathrm{C} / \mathrm{EBP} \beta$ on the activation phenotype of the neoplastic T-cells and expression of CD147, an inductor of matrix metalloproteinases implicated in tumor progression and metastasis in solid tumors. The differential expression of CD147 in ALK+ALCL suggests its involvement in the pathogenesis of ALK+ALCL.

\section{LYS051}

Unusual presentation of anaplastic large cell lymphoma: a case report

Cedna Tomasovic-Loncaric; Gorana Aralica

Department of Pathology University hospital Dubrava, Zagreb, Croatia

Aim: To describe a case of anaplastic large cell lymphoma (ALCL) with an unusual presentation mimicking fever of unknown origin and sepsis.

Case report: A 32 years old male presented with dry cough, nausea, malaise and high fever $\left(41^{\circ} \mathrm{C}\right)$ of 2 weeks duration. Laboratory findings were WBC $12.3 \times 109 / \mathrm{L}$ with neutrophilia (86.2\%), elevated CRP $231.3 \mathrm{mg} / \mathrm{L}$, SE 120, and LDH $206 \mathrm{U} / \mathrm{L}$. Physical examination and chest x-ray showed no apparent abnormalities. Extensive microbiology and serology tests were all negative. During the course patient was deteriorating, with persistent fever and diffuse abdominal pain suggestive of acute abdomen. Laparotomy revealed enlarged mesenteric lymph nodes with diffuse thickening of mesenterial fat.

A lymph node biopsy showed complete effacement of the structure by confluent sheets of medium sized cells with oval and some kidney shaped nuclei. Immunohistochemical staining showed CD30, EMA, CD45, CD2, granzyme B, CD25 and strong ALK nuclear and cytoplasmic expression. The findings were in keeping with ALCL.

Laparotomy was followed by rapid decline; worsening leukocytosis $(20.3 \times 109 / \mathrm{L})$, decrease in platelets $(130 \times 109 / \mathrm{L})$, erythrocytes $(3,77 \times 1012 / \mathrm{L})$, hemoglobin $(102 \mathrm{~g} / \mathrm{L})$, abnormal coagulation tests, hepatic and renal failure. Patient died of respiratory failure (ARDS) 13 days after initial presentation.

Conclusions: Most ALCLs with ALK expression, particularly in children and young adults, show a favorable prognosis and good therapy response. However, cases with an aggressive course have been reported, which might be related to increased expression of cytokines. This case confirms previous observations by showing strong expression of IL-2 receptor (CD25). A misleading combination of 
symptoms supportive of systemic infection without palpable lymph nodes could make the correct diagnosis more difficult to establish and thus delay it. Recognition of this clinical variant of ALCL may facilitate timely diagnosis and therapy.

\section{LYS052}

\section{Analysis of C/EBP $\beta$-dependent miRNA expression} patterns in ALK+ALCL by next generation sequencing

Julia Steinhilber ${ }^{1}$; Irina Bonzheim ${ }^{2}$; Sabine Schäfer ${ }^{2}$; Falko Fend $^{2}$; Leticia Quintanilla-Martinez ${ }^{2}$

(1) Institute of Pathology and Neuropathology, University Hospital Tuebingen (2) Institute of Pathology and Neuropathology, University Hospital Tuebingen, EberhardKarls-University, Germany

Background: ALK+ anaplastic large cell lymphomas (ALCL) show specific overexpression of the transcription factor $\mathrm{C} / \mathrm{EBP} \beta$, as a consequence of NPM-ALK kinase activity.

Aim of the study: The aim of this study was to analyse regulation of miRNA expression by $\mathrm{C} / \mathrm{EBP} \beta$ in ALK +ALCLs, and to identify miRNAs differentially expressed between ALK+ALCLs, ALK- ALCLs and T-cells.

Materials and methods: Three ALK+ALCL cell lines were transduced with $\mathrm{C} / \mathrm{EBP} \beta$ shRNA by lentiviral gene transfer. The $\mathrm{C} / \mathrm{EBP} \beta$ knockdown was quantified by RT-qPCR and Western blot. miRNA expression in ALK+ALCL cell lines with and without C/EBP $\beta$ knockdown, ALKALCL cells and T-cells was analyzed by next generation sequencing using solid-phase amplification with the Illumina Genome Analyzer IIx, to determine differential regulation and expression of miRNAs in ALK+ALCL. The influence of $\mathrm{C} / \mathrm{EBP} \beta$ on the expression of miRNA candidates and the differential expression in ALK+ALCL was validated by RT-qPCR in cell lines and in primary tumors.

Results: Next-generation sequencing analysis resulted in 80 significantly regulated miRNAs after C/EBP $\beta$ knockdown in the three ALK+ALCL cell lines. Three of these miRNAs (miR-181a*, miR-146b-5p, miR-203) were significantly regulated in all three cell lines. These miRNAs show interesting functions and expression patterns. The miR-181 family members are involved in the regulation of differentiation, cell cycle progression and cell survival. The miR-146b-5p has been found to have an oncogenic role in thyroid follicular cells whereas mir-203 is overexpressed in pancreatic adenocarcinoma. The $\mathrm{C} / \mathrm{EBP} \beta$ dependent regulation of these miRNAs was confirmed in two cell lines by RTqPCR. Comparison of the results of the ALK+ALCL cell line SUDHL-1 and T-cells or SUDHL-1 and the ALKALCL cell line Mac-1 revealed 379 and 301 significantly regulated miRNAs, respectively. ALK- ALCL cells and Tcells showed a significant difference in the expression levels of 366 miRNAs. A striking hundred-fold change in expression levels was observed for a few miRNAs of the different signatures. Some of the most strongly regulated miRNAs in ALK+ALCLs were validated in primary human ALK+ and ALK- ALCLs by RT-qPCR. Several of these miRNAs play important roles in tumorigenesis with tumor-suppressing or oncogenic functions.

Conclusions: ALK+ and ALK- ALCL cells show distinct miRNA expression profiles, which at least in part are due to differential regulation by $\mathrm{C} / \mathrm{EBP} \beta$. The differential expression of several candidate miRNAs with putative oncogenic functions is also present in primary lymphoma samples and indicates significant differences in miRNA-dependent regulation of gene expression in ALK+ and ALK- ALCL.

\section{LYS053}

Role of immunohistochemistry to distinguish primary cutaneous anaplastic large cell lymphoma from systemic anaplastic large cell lymphoma. Report of two cases

\section{Mukerrem Safal ${ }^{1}$}

\section{(1) GATA Patoloji AD Ankara Turkey}

Background: Primary cutaneous anaplastic large cell lymphoma (C-ALCL) is an indolent disease and mostly limited to the skin, even though multifocal skin lesions and focal draining lymph node involvement can be seen in a minority of the cases. ALK- systemic anaplastic large cell lymphoma (ALCL) is an agressive entity showing nodal and extranodal involvement with poor prognosis. They must be distinguished from each other for the choice of correct treatment. Aim of the study: To asses the role of immunohistochemistry (IHC) to distinguish C-ALCL from ALK - systemic ALCL.

Materials and methods: Two cases of ALK- ALCL with multiple skin lesions and focal lymphadenopathy at the time of diagnosis are presented. The IHC antibody panel used for differential diagnosis included CD30, ALK, EMA, LCA, Tcell markers (CD2, CD3, CD4, CD5, CD7, CD8), and cytotoxic proteins (TIA-1, grnazyme B, perforin).

Results: To distinguish C-ALCL from systemic ALK ALCL by using IHC in this clinical setting was the main problem but contribution of IHC was very limited.

Conclusions: Limited contribution of IHC to the differential diagnosis of C-ALCL and systemic ALK- ALCL makes the clinical staging the only way to distinguish each other, if both entities are in different stages at the time of diagnosis. 


\section{LYS054}

CD4+ Small T-Cell Lymphoma of the intestine: Report of a case and review of the literature

Larissa Sena Teixeira Mendes; Ayoma Attygalle; David Cunningham; Martin Benson; David Gonzalez de Castro; Andrew Wotherspoon

Royal Marsden Hospital, Fulham Road, London SW3 6JJ, United Kingdom

The majority of T-cell lymphomas arising in the gastrointestinal (GI) tract, particularly those involving the duodenum/small intestine, fall into the category "EnteropathyAssociated T-cell Lymphoma (EATL). EATL is considered to be a tumour of intraepithelial lymphocytes and has two recognised forms of presentation: Type I/ Classical EATL is strongly associated with a celiac disease background, and is composed of T-cells with a CD8 $\pm / C D 4-/ C D 56-$ phenotype. Type II EATL appears to be celiac disease independent, showing monomorphic T-cells with a CD8+/CD4-/CD56+ phenotype.

We report a rare case of a CD4+ small $\mathrm{T}$ cell lymphoma in a 59-year-old female patient who presented with sprue-like symptoms in the absence of celiac disease. Duodenal biopsies showed the lamina propria to be diffusely infiltrated by monomorphic small T-cells and an associated very focal increase in intraepithelial lymphocytes (IELs). These cells had a CD8-/CD4+/CD56- immunophenotype and were clonal by PCR analysis for T-cell receptor (TCR) gene rearrangement. The cells did not display a follicular T-helper phenotype. Biopsies of the stomach and colon showed similar CD4 positive T cell infiltrate and PCR confirmed the presence of identical T cell clones at these sites. PET/CT scan did not identify disease outside the GI tract.

Review of the literature reveal 6 similar cases of intestinal lymphoma composed of small CD4+ T-cells with a generally indolent clinical behaviour. We suggest that this disorder constitutes a new variant of intestinal T-cell lymphomas with a distinct immunophenotype and clinical evolution.

\section{LYS055}

\section{Refractory celiac disease: clinicopathological features of 12 cases}

Fina Climent ${ }^{1}$; Cristina Melendez ${ }^{2}$; Maria José Paúles ${ }^{2}$; Mar Varela $^{3}$; Josep Maria Botargues ${ }^{4}$; Francisco Rodriguez ${ }^{4}$; Enric Condom ${ }^{2}$

(1) Barcelona Cardiovascular Research Center, CSIC-ICCC

(2) Department of Pathology. Bellvitge University Hospital-
IDIBELL. L'Hospitalet de Llobregat (Barcelona (3) Department of Pathology. Catalan Institute of Oncology-IDIBELL (4) Department of Gastroenterology. Bellvitge University Hospital-IDIBELL

Background: Intraepithelial lymphocytes (IEL) in celiac disease $(\mathrm{CD})$ are $\mathrm{CD} 3+$ and $\mathrm{CD} 8+$ and exhibit a polyclonal profile for T-cell receptor (TCR) rearrangement. In contrast, refractory $\mathrm{CD}(\mathrm{RCD})$ may be subdivided into type I (IELs polyclonal and normal phenotype) or type II (IELs monoclonal and/or aberrant phenotype). The aim was to analyze the RCD cases in our institution.

Materials and methods: The clinical, pathological, immunophenotypical and molecular findings of 12 RCD cases were reviewed.

Results: Our series comprised 7 men and 5 women with a median age of 57 years (range: 20-76). One patient had RCDI and 11 patients had RCDII. Six patients presented with no prior clinical or biopsy evidence of gluten sensitivity. Three patients developed RCDII after a history of CD of 12, 60 and 144 months and 1 of them developed Enteropathy associated T-cell lymphoma (EATL). Two patients presented IEL and EATL simultaneously. Phenotype was aberrant in 8 out of 11 and TCR was monoclonal in 11 out of 11 tested cases.

Conclusions: In our series, 6 cases presented as RCDII de novo. The IEL phenotype study and the TCR rearrangement were useful for identifying cases with RCD.

\section{LYS056}

Enteropathy associated T-cell lymphoma: single centre clinicopathological and immunohistochemical study focusing on abberant expression of activation markers and TCR proteins

Piotr Czapiewski ${ }^{1}$; Paul Fisch ${ }^{2}$; Reinhard Marks ${ }^{3}$; Annette Schmitt-Gräff ${ }^{2}$

(1) Department of Pathology, Medical University of Gdansk (2) Department of Pathology, University of Freiburg, Germany (3) Department of Internal Medicine, University of Freiburg

Background: Enteropathy associated T-cell Lymphoma(EATL) is a distinctive clinicopathological entity but knowledge about potential prognostic factors in this disease is very limited.

Aim of the study: Clinicopathological and immunohistochemical analysis of single center EATL cohort focusing especially on stromal components, activation molecules and potential loss of TCR receptors.

Materials and methods: Detailed clinical analysis of a cohort of 21 patients from the southern-west Germany was 
performed. Additionally a broad panel of immunohistochemical stainings including MUM1 $I R F 4$, Blimp-1, FoxP3, TCR $\beta, \operatorname{TCR} \delta, \operatorname{CD} 11 \mathrm{c}$ was done. Univariate statistical analysis of survival was performed.

Results: The study group comprised of 12 males and 9 females, aged 37-86, with the median survival time of 5 months after the initial diagnosis. Following clinical factors turned out to be connected with longer overall survival (OS): age $>60$ years $(p=0,007)$, Ann Arbor Stadium I+II $(p=0,048)$, good general condition $(p=0,0068)$, IPI Stadium I-III $(p=0,004)$ and usage of anthracyclin $(p=0,0504)$. Longer event free survival (EFS) was connected with good general condition $(p=0,023)$ usage of anthracycline $(p=$ $0,047)$.

There were 17 Type I cases (81 \%) and 4 Type II (19\%). EATL type, histological variants of neoplastic cells, perivascular and angiocentric growth pattern as well as tissue eosinophilia did not influence survival. There was a strikingly strong infiltration of the neoplastic infiltrate by CD11c + dendritic cells in $9 / 21$ cases, but it was prognostically irrelevant. MUM1/IRF4 expression was observed in 8/19(42,1\%) Blimp-1 in 5/14(35,7 \%) and FoxP3 in 7/20(35\%), but only FoxP3 positivity was connected with longer EFS. There was a trend toward worse $\operatorname{OS}(p=0,18)$ and $\operatorname{EFS}(p=0,12)$ in Blimp-1 positive cases. Expression of TCR $\beta$ was observed in $4 / 21(19 \%)$, of TCR $\delta$ in $3 / 21(14,3 \%)$ while the majority of cases (14/21), showed loss of expression of both proteins. Median OS and EFS was much longer in TCR $\beta+(38,5$ and 26,5 months, respectively) than in TCR $\delta+(6,4$ and 5,9 months) and TCR $\beta-\delta-(4,4$ and 2$)$ cases, but it did not reach statistical significance probably due to the small number of TCR $\beta+$ positive cases.

Conclusions: Clinical factors provide more relevant prognostic information in EATL than immunohistochemical stainings. The role of FoxP3, TCR $\beta, \delta$, and Blimp-1 requires further studies on larger cohorts.

\section{LYS057}

\section{Extranodal NK/T cell lymphoma, nasal type with expression of $B$ cell markers and without clonal IgH gene rearrangement}

Ana Ponciano'; Francisco Javier Llamas ${ }^{2}$; Ana Lilian Morales $^{2}$; David Valdes ${ }^{2}$; Diana Aguilar ${ }^{2}$; Ricardo Aguilar ${ }^{2}$; Carmen Lome-Maldonado ${ }^{2}$

(1) Hospital General San Juan de Dios, Guatemala (2) Instituto Nacional de la Nutricion SZ, Mexico City, Mexico

Background: Natural Killer (NK)/T-cell lymphomas represent an uncommon group of neoplasms. They are classified as extranodal NK/T cell lymphoma, nasal type, aggressive
NK cell leukemia, chronic lymphoproliferative disorder of NK cells, and NK lymphoblastic leukemia/lymphoma. The prevalence of these neoplasms is by far higher in Asia, Central and South America.

According to the actual classification of the World Health Organization (WHO), extranodal NK/T-cell lymphoma, nasal type, is an aggressive lymphoma characterized by vascular damage, prominent necrosis, cytotoxic phenotype and association with Epstein-Barr (EBV) virus. They commonly involved the upper aerodigestive tract but have also been reported in extranasal sites.

Histopathologically, they are characterized by a diffuse and permeative polymorphic lymphomatous infiltrate, with an angiocentric and angiodestructive growth pattern. In most cases, the neoplastic cells are represented by genuine NKcells but they can also show a cytotoxic T-cell phenotype. There is a strong association with EBV. When considering some of the differential diagnoses, lack of expression of surface CD3, B cell antigens, myeloperoxidase and other lineage markers seems to be important. However, in a small number of NK/T cell lymphoma, aberrant expression of B cell markers has been described. Considering that $\mathrm{B}$ cell markers expression is often taken as an important initial evidence of $\mathrm{B}$ cell differentiation, this can lead to a diagnostic pitfall. In the present study, 25 cases of NK/T cell lymphoma, nasal type were reviewed, looking for expression of B cell markers.

Aim of the study: Determine the expression of B cell markers in 25 cases of NK/T cell lymphoma, nasal type from the Instituto Nacional de Ciencias Médicas y Nutrición, Salvador Zubirán (INNSZ).

Materials and methods: 25 cases of NK/T cell lymphoma, nasal type were reviewed from the pathology archives of the INNSZ from year 2007 to year 2011. The initial evaluation of the specimens was done on hematoxilin and eosin stained slides. Immunohistochemical studies and in situ hybridization for EBER were executed on formalin-fixed, paraffinembedded tissue sections. In cases where expression of B cell markers in neoplastic cells was documented, molecular studies, using polymerase chain reaction (PCR) on paraffin embedded tissue sections, looking for clonal immunoglobulin heavy chain rearrangements, were performed.

Results: According to the morphogical and immunophenotypical criteria of the actual WHO classification of tumours of Haematopoietic and Lymphoid tissues, the diagnosis of extranodal NK/T cell lymphoma, nasal type was confirmed on the 25 cases reviewed. Two cases showed expression of B cell markers. The most relevant of these cases are summarized below.

Case 1: A 74-year-old woman presented with an ulcerated lesion on the left cheek. She was classified as stage I, received chemotherapy based on L-ASPAR/CHOP and radiotherapy. She is alive without disease. The neoplastic cells 
showed positivity to MUM1 and CD79a, focal expression of CD20 and lack of expression of PAX-5.

Case 2: A 75-year-old woman presented with abdominal pain; she was taken to the operating room, small bowel tumour and perforation were evidenced; a segmental resection of the affected area was performed. The patient died from complications of intestinal perforation. The neoplastic cells showed positivity to MUM1, focal expression of CD20 and lack of expression of CD79a and PAX-5. The PCR studies demonstrated no clonal immunoglobulin heavy chain gene rearrangements in either of cases.

Conclusions: The expression of $\mathrm{B}$ cell markers was observed only in two cases of the 25 cases of NK/T cell lymphoma, nasal type reviewed. To date, CD20 expression in NK cell lymphoma has been described in only 4 cases. In one of them, CD79a and MUM1 were also expressed in neoplastic cells. Moreover, in a study of 22 cases of NK/T cell lymphoma, Pongruttipan et al, reported expression of MUM-1 and OCT-2 in neoplastic cells in 5 cases. Meanwhile, Blackolmer et al reviewed 11 cases of NK/T cell lymphoma and found positivity to CD79a in one of them. In relation to CD20 expression, Harinder S Gill et al considered two possible mechanisms that can lead to this phenomenon. The first one is the transformation of a rare cell type that coexpresses CD20 and NK cell markers and the other one regards CD20 positivity just as an aberrant expression due to a neoplastic process. Beyond this, the real significance of B-cell markers expression in NK/T cell neoplasms remains unclear. However, it is important to recognize that these neoplasms may express B-cell markers, particularly CD20 (considering it is a first-line marker to identified B cell lineage) in order to avoid misdiagnosis. Therefore, clinical features and morphological appearance of a lesion coupled to a complete immunophenotyping are highly relevant to establish the correct diagnosis.

\section{LYS058}

\section{CD335 (NKp46) specifically identifies natural killer cells by diagnostic flow cytometry}

Aharon G. Freud ${ }^{1}$; Shuchun Zhao ${ }^{2}$; Sibing Wei $^{3}$; Gary M. Gitana $^{3}$; Yasodha Natkunam ${ }^{3}$; Susan K. Atwater ${ }^{2}$

(1) Stanford University (2) Stanford University School of Medicine (3) Stanford University Medical Center, USA

Background: Natural killer (NK) cells are large granular lymphocytic (LGL) effector cells of the immune system that mediate MHC-independent cytotoxicity against virallyinfected and malignant cells and also produce a host of inflammatory cytokines that modulate both innate and adaptive arms of the immune system. Because of their important roles in immunity, the identification, enumeration, and immunophenotypic characterization of NK cells by diagnostic flow cytometry is routinely performed on clinical samples. Although to date there are no known NK-specific surface antigens, NK cells may be identified and enumerated based on their lack of expression of non-NK lineageassociated antigens (e.g. CD3, CD20) combined with their positive expression of pan-NK markers such as CD16 and CD56. However, the latter are not specific to the NK lineage, and often they may be expressed on non-NK neoplastic proliferations, which may present a diagnostic challenge. CD335 (NKp46) is a surface activation receptor that is normally expressed on all mature NK cells in human peripheral blood and was evaluated in our clinical laboratory to assess its potential utility in the diagnostic setting.

Aim of the study: To determine the sensitivity and specificity for identifying NK lineage cells using an anti-CD335 monoclonal antibody in the clinical diagnostic flow cytometry setting.

Materials and methods: A 6-color cocktail containing CD3 APC-H7, CD16 V450, CD45 PerCP, CD56 FITC, CD161 (NKRP1A) APC, and CD335 PE antibodies was optimized and evaluated on patient specimens submitted for diagnostic flow cytometry. Patient samples were processed and analyzed by clinical laboratory scientists. Populations were gated using CD45 expression and forward and side scatter characteristics, and NK cells were identified as CD3(-) events within the lymphocyte gate and expressing at least one of the following surface antigens: CD16, CD56, and CD161. Diagnoses were made using WHO 2008 criteria.

Results: A total of 668 specimens, including blood $(n=$ $187)$, bone marrow $(n=326)$, and lymph node $(n=95)$, were stained with the 6-color antibody cocktail. Diagnoses included a broad spectrum of reactive conditions as well as B cell, T cell, plasma cell, and myeloid neoplasms. In 636 of 653 cases $(97 \%$ ) with detectable CD335 expression, the latter was restricted to NK cells, with variability of staining intensity observed in a significant proportion of cases. In contrast, other NK-associated antigens were often expressed by subsets of T cells (CD56 and CD161), monocytes (CD16 and CD56), neoplastic plasma cells (CD56), and non-hematolymphoid neoplasms (CD56). CD335 was never detected on B cell, monocytic, myeloid, or plasma cell populations. However, nine of 666 cases demonstrated minute populations of nonneoplastic/non-atypical T cells ( $<5 \%$ of total T cells) with dim CD335 expression. In addition, three atypical $\mathrm{T}$ cell proliferations (each suspicious for T-LGL leukemia) and 5 mature $\mathrm{T}$ cell neoplasms demonstrated uniform dim CD335 expression. The latter included three T-LGL leukemias, one peripheral $\mathrm{T}$ cell lymphoma, not otherwise specified, and one anaplastic large cell lymphoma, ALK positive. Five atypical NK lymphocytoses suspicious for chronic lymphoproliferative disorder of NK cells showed marked reduction in CD335 intensity staining ( $4 / 5$ cases) or completely lacked detectable CD335 
(1/5 cases). Two EBV positive mature NK neoplasms showed positive albeit dim CD335 expression. Overall, the sensitivity and specificity of CD335 labeling of NK lineage cells was $99.7 \%$ and $97.9 \%$, respectively.

Conclusion: The 6-color flow cytometry cocktail that we utilized detects NK cell derived proliferations with very high sensitivity and specificity. We show that CD335 is thus far the most lineage-specific marker for NK cells, and assessment of its expression may be useful to identify and enumerate NK cells in clinical specimens. The observation that CD335 may be aberrantly expressed on neoplastic T cells further supports its potential utility in diagnostic flow cytometry. Studies are ongoing to evaluate the diagnostic utility of CD335 expression by immunohistochemistry.

\section{LYS059}

Epstein-Barr virus (EBV) associated NK/T cell lymphoproliferative disorders (EBV T/NK-LPD) in non-immunocompromised Mexican adults with an indolent/chronic clinical course. Report of three cases

Carmen Lome-Maldonado ${ }^{1}$; Yvette Neme-Yunes ${ }^{1}$; David Valdés ${ }^{1}$; Fabian Amador-Medina ${ }^{1}$; Marcela Deffis-Court ${ }^{2}$; Ana E. Ponciano-Castellanos ${ }^{2}$; Francisco Javier Llamas ${ }^{1}$; Ana Lilia Morales ${ }^{2}$; Diana Aguilar ${ }^{3}$; Ricardo Aguilar ${ }^{1}$; Morelos Villaseñor ${ }^{1}$; Arturo Angeles ${ }^{1}$

\section{(1)INCMNSZ Mexico City (2) SPMD (3) PhD}

Background: $\mathrm{EBV}+\mathrm{T} / \mathrm{NK}-\mathrm{LPD}$ was described in children and young adults with a broad clinical spectrum that includes aggressive clinical forms like systemic $\mathrm{EBV}+\mathrm{T} /$ NK-LPD, characterized by a clonal proliferation of EBVinfected T-cells with an activated cytotoxic phenotype, either associated or not with hemophagocytic lymphohistiocytosis (HLH). Both clinical disorders EBV+T/NK-LPD and $\mathrm{HLH}$ are associated with an aggressive clinical course. In the spectrum of chronic or indolent forms, the EBV+T/ NK-LPD includes severe chronic active EBV infection (CAEBV), hydroa vacciniforme and hypersensitivity to mosquito bites (HMB); the two latter involving the skin and presenting a more chronic clinical course. The term CAEBV should be applied to systemic LPDs that arise during primary EBV infection that persists for over 6 months, mainly characterized by fever, lymphadenopathy and splenomegaly. EBV infected cells are either CD4/NK/ CD8 or gamma/delta T cells, with a cytotoxic phenotype. The evidence of clonality in these proliferations is mostly related with an aggressive clinical course that eventually progress to evident lymphomas. Nonetheless, clonality is also related occasionally to non- aggressive forms of CAEBV or cutaneous EBV NK/T LPD.
Materials and methods: Three cases of EBV-NK/T LPD were selected from the files of the INNSZ. Clinical data were obtained, morphological, immunohistochemical and molecular analysis, including EBER in situ hybridization were performed. T cell receptor (TCR) genes rearrangement was analyzed in two cases.

Results: According to the 2008 EBV meeting, these three cases were classified as EBV+NK/T-LPD, with the following peculiar features:

Case 1. A 16-year-old female presented with dysphagia, odynophagia, pharyngitis, fever, malaise, diaphoresis, cervical lymphadenopathy, and hepatomegaly. Bone marrow was infiltrated by a strikingly atypical CD8+ T-cell proliferation with activated cytotoxic phenotype, EBER+. Spontaneous remission without treatment was documented 2 weeks after the diagnosis and the patient is alive and disease free after 11 years of follow-up.

Case 2. A 50-year-old female presented with slow growing, non-healing painful perianal and vulvar papular ulcerated lesions, without systemic symptoms. Histopathologic diagnosis: $\mathrm{EBV}+\mathrm{NK} / \mathrm{T}-\mathrm{LPD}$ with exclusive cutaneous involvement and without $\mathrm{HV}$ clinical features. CD56+, CD2+, CD7+, TIA1+, GRAN+, PERF+, CD3+/-, CD4+/-, CD8-/+, CD5+, CD20-, EBER+. Clonality for TCR was demonstrated. The patient was treated with radiotherapy and at 18 months of follow-up is still alive with flares and remissions of the cutaneous lesions.

Case 3. A 16-year-old female with papulo-vesicular lesions associated with mosquito bite, fever, weight loss, bone pain, splenomegaly, hemophagocytic syndrome, and a NK cell lymphocytosis (leukocytes $12700 \times \mathrm{ml}$, with $74 \% \mathrm{NK}$ lymphocytes) CD2 $95 \%$, CD56 $74 \%$, CD16 $76 \%$ by flow cytometry. Histopathological diagnosis: EBV+NK/T-LPD (CAEBV type). Sites affected were bone marrow, lymph nodes, spleen, liver and nasal epithelium with a discrete NK, CD4, and CD8 infiltratation that was EBV related but mainly infected CD4 cells. Clonality for TCR was demonstrated. The patient received treatment with cyclosporine and she is alive and asymptomatic after 3 months of follow-up.

Conclusions: EBV $+\mathrm{NK} / \mathrm{T}-\mathrm{LPD}$ includes a wide variety of morphological and clinical aspects, with overlapping terminology in the medical literature due to the wide clinical and pathological spectrum of the lesions. The cases that we described here are intended to give useful information regarding their peculiar characteristics. EBV+NK/T-LPD is not exclusive of childhood; it can affect adults in the fourth and fifth decade of life and some cases do have a chronic and indolent course, in contrast to what had been initially described. EBV $+\mathrm{NK} / \mathrm{T}-\mathrm{LPD}$ can display EBV infection with CD4 and CD8 and/or NK cells. A worse prognosis is suggested for cases associated with CD4/EBER, while the coinfection CD56/EBER is associated with an apparently 
better prognosis. This is shown in case number 2, which illustrates an indolent pure cutaneous involvement affecting non-light exposed areas. EBV+NK/T-LPD may exhibit either a chronic clinical and indolent course, (cases 2 and 3 ) or an acute one with spontaneous remission (case 1). This appears to be dependent on the immune integrity of the host rather than on the morphology, phenotype or clonality of the lesion. This work could provide useful elements for the specific definition of this group of lymphoproliferative disorders, however the biologic significance should be clarified in the future.

\section{LYS060}

Pediatric extranodal T/NK cell lymphoma; case report and review of literature

Howayda ABD EL ALL ${ }^{1}$; Dalia Ibrahim ${ }^{2}$

(1) University of Santa Cruz do Sul (2) Ein-Shams University, Dept Pediatrics

Background: Extranodal NK/T cell lymphoma is an adult neoplasm more prevalent in Asia.

Aim of the study: To present a case of NK/T-cell lymphoma in a child.

Materials and methods: Skin and bone marrow trephine biopsies were obtained and processed.

Results: A 14-year-old girl presented with generalized skin nodules and papules and bilateral chest nodules that resolved after corticosteroids. The skin lesion was composed of a mixture of atypical lymphocytes and histiocytes surrounding thin and thick walled blood vessels and adnexae, sparing a thinned-out epidermis. Up to $50 \%$ of the bone marrow was infiltrated by the same neoplastic lymphocytes. The neoplastic lymphocytes were positive for CD3, CD56 and granzyme B while EBV LMP, and EBER were negative.

Conclusions: Extranodal NK/T cell lymphoma, in this pediatric case, showed membranous positivity for $\mathrm{CD} 3$ and negativity for EBV (IHC and ISH).

\section{LYS061}

Correlation between immunophenotype profile, Epstein-Barr-Virus (EBV) infection and morphological features in a Mexican cohort of 56 cases with T-cell lymphoma and Extranodal NK-cell lymphoma

Francisco Llamas-Gutiérrez ${ }^{1}$; Ana Lilia Morales-Leyte ${ }^{1}$; David Valdés ${ }^{1}$; Jazmín De Anda-González ${ }^{1}$; Braulio Martínez-Benítez ${ }^{1}$; Morelos Villaseñor-Aguirre ${ }^{1}$; Diana Aguilar-Leon ${ }^{1}$; Ricardo Aguilar-Guadarrama ${ }^{1}$; Carlos OrtizHidalgo $^{2}$; Cesar Lara-Torres ${ }^{2}$; Elizabeth Romero ${ }^{3}$; Ivette
Neme-Yunes ${ }^{1}$; Carmen Lome-Maldonado ${ }^{1}$

(1)Instituto Nacional de Ciencias Médicas y Nutrición Salvador Zubirán (2) Centro Medico ABC Mexico (3) Centro Medico Nacional Siglo XXI Mexico

Background: Mature T-cell lymphomas are uncommon neoplasms, representing 10-15\% of Non-Hodgkin Lymphomas (NHL). In the actual WHO classification, 4 groups are recognized: Extranodal NK/T-cell lymphoma nasal type (ENKL), angioimmunoblastic T-cell lymphoma (AITCL), peripheral T-cell lymphoma (PTCL) and anaplastic large T-cell lymphoma (ALCL). Regarding the immunophenotype profile, four groups have been proposed in the literature: regulatory $\mathrm{T}$-cell phenotype (T-reg) that shows positivity to CD4, CD25 and FOXP3. This phenotype has been rarely described in neoplastic cells, mainly associated with HTLV-1 infection. Activated-cytotoxic profile (AC): $\mathrm{CD} 8+$, granzyme+, perforin+, TIA1+. This phenotype has been associated with a worse prognosis. The follicular phenotype (T-FH) corresponds to lymphomas arising from germinal center expressing CD10, PD1, ICOS, CXCL13; most of the ATCL fit in this category. It is known that a significant number of lymphomas present a variable positivity to EBV. CD30 expression has been associated with a worse prognosis.

Aim of the study: To analyze IHC profiles of 56 cases of Non-Hodgkin lymphoma including PTCL, ENKL, AITLC, ALCL, and describe EBV infection and their clinical course. Materials and methods: A 5-year retrospective study was performed (2007-2011) in three Hemato-Oncological Institutions in Mexico City. A total of 56 cases with a T-cell NHL diagnosis were included. Morphological analysis, immunohistochemical (IHC) studies and clinical features were performed for each case. IHC analysis included: CD3, CD20,CD4,CD8,CD5,TIA1,PERFORIN,GRANZYME, CD56,CD10,PD1,CXCL13,ICOS,CD30, ALK,CD25, FOXP3. EBER in situ hybridization was also performed. For statistical analysis, Kaplan-Meyer with log-rank test was used to evaluate survival.

Results: The 56 cases were morphologically classified into 4 groups and were correlated with the IHC profile. Unclassifiable phenotype (UC) included lymphomas that did not fit in any of the 3 IHC profiles previously described. ENKL ( 22 cases): 20 cases were found with an AC phenotype and $2 \mathrm{UC}$; all cases were EBV related.

PTCL (22 cases): 14 cases presented UC phenotype, 3 AC, 3 T-reg, 2 T-FH. EBV was found in $37.5 \%$.

AITL ( 8 cases): All cases presented a T-FH phenotype, $75 \%$ were EBV related.

ALCL (4 cases): 2 UC, 1 AC, 1 T-fh, EBV was negative in all cases. CD30 was mainly expressed in AITL and ALCL, although no clinical correlation was found. 
Conclusions: In this series, PTCL and ENKL were the most common histologic subtypes; this was probably determined by ethnicity. We found EBV-association to be stronger in ENKL, which showed a worse prognosis. $31 \%$ of PTCL were EBV-positive, similar to what is reported in the literature. Curiously, $5.3 \%$ of the cases presented T-reg phenotype, which is slightly higher than what is reported in the literature. PCR for HTLV-1 was performed in only one PTCL case with T-reg profile, and was reported negative. Regarding FOXP3 expression, its positivity did not show statistical significance for survival $(p=0.95)$. All AILT expressed T-FH phenotype, equal to what is reported in the literature. $75 \%$ were EBV-positive. Morphologic subtype was shown to significantly affect survival $(p=0.002)$. ENKL showed the worst prognosis of all the groups, followed by PTCL. No statistical significance was shown when subdividing IHC profile. EBV-positivity was found to be associated with inferior survival than EBV-negative cases; nonetheless, most of these cases were ENKL. The IPI was not associated with any morphological pattern or IHC profile in particular. Although the immunophenotype expression profile did not show prognostic significance in this series, the immunophenotype subgroups (specifically for $\mathrm{T}-\mathrm{fh}$, and $\mathrm{T}-\mathrm{AC}$ ) were associated with specific clinical morphological entities, suggesting that these subgroups may represent different biological entities. These differentiation profiles were associated with particular clinicopathological features but were not helpful to predict outcome in T-cell lymphomas in this study.

\section{LYS062}

\section{C-MYC Translocation Frequency of Germinal Center B Cell Like Type Diffuse Large B Cell Lymphoma and Non-Germinal Center B Cell Like Type Diffuse Large B Cell Lymphoma}

Bahar Akkaya; Senay Arikan; Mualla Ozcan; Irem Hicran Ozbudak; Aysen Timuragaoglu

Akdeniz University School of Medicine Pathology Department

Background: Diffuse large B-cell lymphoma (DLBCL) is the most common type of malignant lymphoma and constitutes $30-40 \%$ of adult non-Hodgkin's lymphomas in western countries. DLBCL is a heterogeneous disease group with varied clinical courses, histology, molecular and cytogenetic characteristics.

Aim of the study: Recent studies showed that DLBCL cases with gene expression profiles similar to germinal center B cells had much better prognosis than DLBCL cases with gene expression profiles resembling activated B cells. 3 antibody panel (CD 10, BCL6, MUM 1) of immunohistochemical stains can be used to subclassify DLBCL into prognostically significant germinal center B-cell like (GCB) DLBCL and activated B-cell like (ABC) DLBCL subtypes. Both CD 10 and BCL6 are considered as germinal center markers while MUM 1 is expressed in activated Bcells and plasma cells. C-MYC translocations are characteristic of Burkitt lymphoma, it also has been identified 5$15 \%$ cases of DLBCL. The C-MYC gene at $8 \mathrm{q} 24$ is involved in three translocations, most commonly $t(8 ; 14)$ (q24; $\mathrm{q} 32)$ and less often $t(2 ; 8)(\mathrm{p} 12 ; \mathrm{q} 24)$ and $t(8 ; 22)(\mathrm{q} 24 ; \mathrm{q} 11)$.

Materials and methods: In the present study, we evaluated the expression patterns of CD 10, BCL6 and MUM 1 by immunohistochemistry on paraffin-embedded tissues from 50 DLBCL patients. Gene status was examined by using Fluorescence in situ hybridization (FISH) and the results were analysed in the context of GCB and non-GCB type of DLBCL.

Results: Median age of patients is 53.16. There were 24 women (48 \%) and 26 men (52\%) patients. Tumours were located at lymph nodes $62 \%$ of patients. There were statistically significant differences in the expression of CD 10 (8/ 16 vs 4/28), BCL6 (10/16 vs 5/28), MUM 1 (0/16 vs 28/28) between GCB type and non-GCB type DLBCL. C-MYC translocation was observed in \%18.6 (8/43). C-MYC translocation was more common in ABC type DLBCLs (24\%) than in GCB types (13,3\%). C-MYC rearrengement was determined 2 cases of GCB type and 6 cases of non-GCB type DLBCL. Presence of $t(8 ; 14)(\mathrm{q} 24 ; \mathrm{q} 32)$ is demonstrated only two cases within non-GCB type DLBCL subgroup. No relationship between C-MYC translocation and/or age and gender was observed. There was no significant correlation between C-MYC expression and DLBCL subtype.

Conclusions: Cases in which clinical, morphological and immunohistochemical diagnosis are not adequate to discriminate DLBCL-BL, cytogenetic studies are beneficial. In this way, not only the patient will be able to reach the correct diagnosis but also get the effective treatment.

\section{LYS063}

Quantitative Immunohistochemistry Reveals the Signature of Active B Cell Receptor Signaling in Diffuse Large B Cell Lymphoma

Agata M. Bogusz ${ }^{1}$; Richard H.G. Baxter ${ }^{2}$; Treeve Currie ${ }^{1}$; Papiya Sinha ${ }^{1}$; Jeffery L. Kutok ${ }^{1}$; Scott J. Rodig ${ }^{1}$

(1) Brigham and Women's Hospital (2) Yale University

Background: B cell receptor (BCR) mediated signaling is important to the pathogenesis of a subset of diffuse large $\mathrm{B}$ 
cell lymphomas (DLBCL) and the BCR-related kinases SYK and BTK have recently emerged as potential therapeutic targets. Successful application of these targeted therapies requires quantitative assessment of BCR mediated signaling in clinical specimens.

Aim of the study: We sought to identify a signature of activated BCR signaling in DLBCL to aid in the identification of tumors that are most likely to respond to BCRpathway inhibition.

Materials and methods: We applied semi-automated quantitative immunofluorescence (qIF) using antibodies to phosphorylated forms of proximal BCR signaling kinases LYN, SYK and BTK and antibody to BCR-associated transcription factor FOXO1 on BCR-crosslinked formalin-fixed paraffinembedded (FFPE) DLBCL cell lines as a model system and on a cohort of 60 FFPE patient DLBCL specimens.

Results: A robust signature based on phospho-specific antibodies to the proximal BCR signaling molecules LYN, SYK and BTK detected active BCR signaling in 32/60 (53 \%) of the examined tumors. Further analysis revealed increased nuclear exclusion of the distal BCR signaling-associated forkhead transcription factor FOXO1 among DLBCL with qIF evidence of active BCR signaling compared to those without $(p=0.0001)$. Nuclear exclusion of FOXO1 was also detected in a subset of DLBCL without evidence of proximal BCR signaling suggesting that alternative mechanisms for PI3K/AKT activation may mediate FOXO1 subcellular localization in these cases.

Conclusions: We report the successful detection of active BCR signaling by quantitative immunofluorescence (qIF) in formalin-fixed paraffin embedded DLBCL cell lines and a cohort of 60 patient specimens. This study establishes the feasibility of detecting BCR activation in primary FFPE biopsy specimens of DLBCL. It lays a foundation for future dissection of signal transduction networks in DLBCL and provides a potential platform for selecting individual tumors as candidates for novel therapies targeting the BCR pathway.

\section{LYS064}

18F-FDG uptake in primary gastric malignant lymphoma correlates with glucose transporter 1 expression and tumor aggressiveness

Yuko Watanabe ${ }^{1}$; Hiroaki Suefuji1; Yasumitsu Hirose1; Hayato Kaida ${ }^{1}$; Chikayuki Hattori ${ }^{1}$; Hidehiro Eto ${ }^{1}$; Gen Suzuki ${ }^{1}$; Etsuyo Ogo ${ }^{1}$; Masatoshi Ishibashi ${ }^{1}$; Takashi Okamura $^{2}$; Koichi Ohshima ${ }^{3}$; Naofumi Hayabuchi1

(1) Department of Radiology Kurume University School of Medicine (2) Department of Hematology and Oncology, Kurume University School of Medicine (3) Department of Pathology, Kurume University School of Medicine
Background: PET is useful modality for staging and response evaluation in primary gastric lymphoma (PGL). However, the implication of [18F]-2-fluoro-2-deoxy-D-glucose (18F-FDG) uptake in PGL at the first diagnosis has not been reported.

Aim of the study: The purpose of this study was to assess the relationship between 18F-FDG uptake and the expression of facilitative glucose transporters (GLUTs), as well as tumor aggressiveness in primary gastric lymphoma.

Materials and methods: A total of 23 patients with primary gastric lymphoma were enrolled, including 9 with diffuse large B-cell lymphoma (DLBCL), 7 with high-grade mucosa-associated lymphoid tissue (MALT) lymphoma, and 7 with low-grade MALT lymphoma. The expression levels of GLUT1, GLUT3, GLUT4, and HK II were evaluated according to the percentage of area positive for expression as determined by immunohistochemistry, with a score of 1-4. Maximum standardized uptake values were used for evaluation of tumor 18F-FDG uptake.

Results: Maximum standardized uptake values significantly correlated with GLUT1 expression ( $p=0.02$, Spearman's rank correlation test). Pathological malignant potentials (low-grade MALT lymphoma, high grade MALT lymphoma, and DLBCL) also correlated with maximum standardized uptake values ( $p=0.01$, Kruskal-Wallis test).

Conclusions: We determined that $18 \mathrm{~F}-\mathrm{FDG}$ uptake in primary gastric lymphoma is significantly related to the expression of GLUT1and histological grade of the tumor in primary gastric lymphoma.

\section{LYS065}

Follicular Lymphoma in Taiwan: A low frequency of $t(14 ; 18)$ with grade $3 \mathrm{~A}$ tumors more related to low-grade than to grade 3B tumors

Shih-Sung Chuang; Sheng-Tsung Chang; Yi-Hsuan Lu; Szu-Yin Kuo; Hongxiang Liu; Shu-Hui Lin; Yen-Chuan Hsieh

Chi-Mei Medical Center, Tainan, Taiwan

Background: Follicular lymphoma (FL) is a common lymphoma type in the West with chromosomal translation $t$ $(14 ; 18) /$ IGH-BCL2 in up to $90 \%$ cases. The incidence of $\mathrm{FL}$ is increasing in the East Asia in recent years.

Aim of this study: To characterize the immunophenotype and genetic alternations of FL in Taiwan.

Materials and Methods: We retrospectively investigated a consecutive series of FL in a single institute in Taiwan from year 2000 to 2010 using immunohistochemistry and fluorescence in situ hybridization (FISH).

Results: Of the 93 cases studied, the median age was 57 (range, 26-89), 43 (46\%) patients were males, 77 (83\%) 
tumors were de novo and $84(90 \%)$ were nodal. Fifty nine (63\%) tumors were low-grade (LG; $42 \%$ grade $1,22 \%$ grade 2 ) and $34(37 \%)$, high-grade (HG; $24 \%$ grade $3 \mathrm{~A}$ and $13 \%$ grade 3B). CD10, bcl-2, bcl-6 and MUM1 were expressed by $73 \%, 94 \%, 97 \%$ and $34 \%$ tumors, respectively. FISH showed IGH, BCL2 and BCL6 rearrangement in $59 \%, 47 \%$ and $11 \%$ cases, respectively. MYC was rearranged in $5 \%$ grade $3 \mathrm{~A}$ and $25 \%$ grade $3 \mathrm{~B}$ tumors. The translocation partner of all BCL2 rearranged tumors was IGH, with IGH/BCL2 translocation identified in $47 \%$ (42/ 90 ) cases (63\% in LG and $18 \%$ in HG). As compared to HG tumors, LG tumors was enriched with a $\mathrm{CD} 10+/ \mathrm{bcl}-2+/$ MUM1- phenotype and were more frequently associated with BCL2 but less commonly with BCL6 rearrangement. Grade 3A FLs were more related to grade 3B than to $\mathrm{LG}$ tumors in immunophenotype (CD10, bcl-2 and MUM1) and genetic aberrations (IGH, BCL2 and BCL6 rearrangement). There was no statistically significant difference between grade 1 vs. 2 , between grade $3 \mathrm{~A}$ vs. $3 \mathrm{~B}$, or between nodal vs extranodal tumors in immunophenotype or FISH (IGH, $\mathrm{BC} 2, \mathrm{IGH} / \mathrm{BCL} 2$ and BCL6) findings.

Conclusions: As compared to the very strong association of $t(14 ; 18) / \mathrm{IGH}-\mathrm{BCL} 2$ with FL in the West, the frequency in Taiwan is among the lowest in the world. Additional studies to identify the genetic alterations of the $t(14 ; 18) /$ IGH-BCL2 translocation-negative cases and their clinicopathological significance are warranted.

\section{LYS067}

Prognostic significance of Hypoxia-Inducible Factor-1 $\alpha$ and Vascular Endothelial Growth Factor expression in patients with Diffuse Large B-cell Lymphoma treated with Rituximab

James Powell ${ }^{1}$; Stefan Dojcinov ${ }^{2}$; Eve Gallop-Evans ${ }^{1}$

(1) Velindre NHS Trust (2) University Hospital of Wales, UK

Background: Hypoxia is a common feature of rapidly growing tumors and cellular responses to hypoxia are fundamental to the behavior of cancer cells. Hypoxia inducible factor- $1 \alpha(\mathrm{HIF}-1 \alpha)$ is a crucial transcription factor governing this process and transcribes many hypoxia related genes including vascular endothelial growth factor (VEGF). In this study we evaluated the association between HIF- $1 \alpha$ and VEGF expression and their prognostic significance in diffuse large B cell lymphoma (DLBCL).

Aim of the study: We aim to characterize the association between HIF- $1 \alpha$ and VEGF expression in a sample of patients diagnosed with DLBCL and to investigate the prognostic significance of this. We also aim to evaluate the prognostic significance of this expression in the subgroup of patients treated with Rituximab.

Materials and methods: Tissue microarrays were constructed from biopsies of 78 patients diagnosed with DLBCL and treated between 2001 and 2006. Immunohistochemical expression of HIF- $1 \alpha$ and VEGF was studied and results were correlated with the clinicopathological factors and prognostic outcome data.

Results: HIF- $1 \alpha$ and VEGF were expressed in $67 \%$ and $84 \%$ of patients respectively, whilst $64 \%$ of patients demonstrated co-expression of the two proteins. A significant positive association was demonstrated between HIF-1 $\alpha$ and VEGF expression $(P<0.001)$. When outcome was analyzed according to Rituximab treatment, HIF- $1 \alpha$ positive patients treated with rituximab demonstrate a trend toward improved OS and PFS with 5-year OS of $72 \%$ for those receiving rituximab versus $65 \%$ for patients not receiving Rituximab and, similarly, 5 -year PFS of $76 \%$ versus $57 \%$.

Conclusions: The results confirm the significant association between HIF- $1 \alpha$ and VEGF expression and suggest that HIF- $1 \alpha$ expression may have a prognostic role in a subset of patients with DLBCL treated with Rituximab.

\section{LYS068}

Effect of changes in color filtering parameters on microvasculature measurement results in Diffuse Large B-cell Lymphoma

Tibor Mezei; Ioan Jung; Egyed-Zs. Imre

University of Medicine and Pharmacy Targu Mures, Roumania

Background: Tumor microenvironment of lymphoma has gained interest since stromal gene signatures may have prognostic and predictive value. Among stromal components, angiogenesis and microvascularity has been intensively studied. For quantification of tumor microvascularity digital morphometry methods are frequently applied since they deliver precise and highly reproducible results in a mathematical format.

Aim of the study: The purpose of our study was to study the degree in which user intervention may influence the results in the assessment of microvasculature of diffuse large B-cell lymphoma (DLBCL) when using a hue/saturation/ brightness color filtering method.

Materials and methods: We used 10 randomly selected patients diagnosed with DLBCL. From each case, FFPE tissue sections were stained for CD34 (immunohistochemistry with DAB chromogen). For image analysis, we used ImageJ software and its macro language. We analyzed the microvasculature (CD34 positive area percentage) using hue/saturation/brightness (HSB) based color filtering. We defined gold standard parameters under visual control, after 
which we altered several analysis parameters in order to see whether these influenced analysis results.

Results: Changing filtering settings (values of hue, saturation) we found that the end results, as compared to the initial gold standard, were significantly altered by saturation changes and not significantly (within a range of $12,5 \%$ ) by changes of hue.

Conclusions: We conclude that the initial parameter settings used in HSB-based color filtering are essential in achieving reliable and reproducible results. Only this way can we assure that these digital morphometry methods are accurate in the study of lymphoma microvasculature.

Acknowledgements: This publication is partly supported by the Sectorial Operational Programme Human Resources Development (SOP HRD), financed from the European Social Fund and by the Romanian Government under the contract number POSDRU 60782.

\section{LYS069}

\section{Expression of Stathmin 1 in mantle cell lymphoma}

Alberto Zamò ${ }^{1}$; Jennifer C Paterson ${ }^{2}$; Alice Parisi ${ }^{1}$; Marco Chilosi $^{1}$; Teresa Marafioti ${ }^{2}$

(1) University of Verona, Italy (2) University College London, UK

Background: The pathogenesis of mantle cell lymphoma (MCL) is still incompletely understood. In previous work, our group used conventional 2D-PAGE proteomics to identify up- and down-regulated proteins in MCL. Stathmin 1 was detected among the most up-regulated proteins, and it elicited our interest because it maps to $1 \mathrm{p} 36$, a frequently altered locus in MCL, and a breakpoint region in MCL cell line MAVER-1. A correlation of Stathmin 1 expression with prognosis and response to therapy has been noted in other neoplastic disorders. Recent data show that the activity of Stathmin 1 might be important as a downstream effector of the immunological synapse. Altered B-cell receptor signaling has been demonstrated in MCL by several groups, including ours, and Stathmin 1 might be implicated in this process.

Aim of the study: To investigate the expression of Stathmin 1 in a series of MCL cases and xenotransplanted cell lines. Materials and methods: The expression of Stathmin 1 was analyzed in a series of 49 MCL cases and three xenografts of MCL cell lines (MAVER-1, Jeko-1, UPN-1) by means of immunohistochemistry. Percentages of positive neoplastic cells as well as staining intensity and pattern were recorded. An arbitrary threshold set at $30 \%$ positive neoplastic cells was used to define cases as positive or negative.

Results: The percentage of positive neoplastic cells varied between $5 \%$ and $100 \%$. Using a threshold set at $30 \%$, we have detected expression of Stathmin 1 in $42 / 49$ cases $(86 \%)$. All the three implanted tumors showed a strong and diffuse staining in all cells. In reactive lymphoid tissues, Stathmin 1 expression was limited to germinal centers, while mantles were completely negative.

Conclusions: These data validate our previous findings, and support a role for Stathmin 1 in the pathogenesis of MCL. Since Stathmin 1 is totally negative in reactive follicle mantles, we also propose the use of Stathmin 1 in the diagnostic immunohistochemical panel for MCL.

\section{$\underline{\text { LYS070 }}$}

C-MYC Rearrangements Detectable with Breakapart Probes Characterize a Subset of Diffuse Large-B Cell Lymphoma Patients with Poor Outcome: A Report from an International DLBCL Rituximab-CHOP Consortium Program Study

Alexander Tzankovi ${ }^{1}$; Marc Gerhard ${ }^{1}$; Zijun Y. XuMonette $^{1}$; Carlo Visco ${ }^{1}$; Stephan Dirnhofer ${ }^{1}$; L. Jeffrey Medeiros $^{2}$; Ken H. Young ${ }^{2}$; on behalf of an International DLBCL Rituximab-CHOP Consortium Program Study ${ }^{1}$

(1) Institute of Pathology, University Hospital Basel, Switzerland (2) The UT MD Anderson Cancer Center, USA

Background: Diffuse large-B cell lymphoma (DLBCL) risk assessment is based on the international prognostic index (IPI), which takes into account patient- and laboratory related parameters. Gene expression profiling (GEP) can stratify DLBCL patients into germinal center B-cell (GCB) and activated $\mathrm{B}$-cell $(\mathrm{ABC})$ subtypes with different prognoses. These groups remain of prognostic importance with the addition of rituximab (R) to cyclophosphamide, hydroxydaunorubicin, vincristine, and prednisone (CHOP) chemotherapy. The prognostic role of C-MYC gene aberrations in DLBCL patients treated with R-CHOP is still debatable.

Aim of the study: We addressed the prognostic significance of C-MYC gene abnormalities determined by interphase fluorescence in situ hybridization (FISH) in a large, multicenter study of de novo DLBCL patients treated with RCHOP.

Materials and methods: 592 DLBCL patients were included: 344 males (mean age 60 years), 248 females (mean age 63 years). 485 were homogenously treated with R-CHOP, while 107 were given R-CHOP-like regimens, i.e. schemes adopting different anthracyclines. Radiotherapy was administered to 165 patients. 379 lymphomas were primarily nodal, 202 were extranodal and in 11 patients the primary tumor location could not be determined. 217 patients had low IPI (0-1), 125 had low-intermediate IPI (2), 105 had high-intermediate IPI (3) and 78 had high IPI (4-5). 455 
DLBCL were characterized by GEP and all cases were classified as GCB or ABC utilizing GEP and the Visco's algorithm. Different morphological (mitotic count, starry sky pattern) and phenotypical characteristics (proliferative activity assessed by Ki-67 staining, myc protein expression) were considered. C-MYC gene abnormalities were assessed using break-apart- (BAP) and IGH/C-MYC double-fusion (DFP) probes. Descriptive data analyses, statistical correlations and survival studies were performed using Spearman-, Kruskal-Wallis-, Kaplan-Meier-, log-rank and Cox regression tests.

Results: After R-CHOP therapy, 439 (74 \%) patients achieved complete remission, $82(14 \%)$ had a partial remission, $29(5 \%)$ stable disease and $42(7 \%)$ disease progression. Within the mean observation period of 39 months (median 36 months), there were 251 recurrences, 230 (39\%) patients died, thereof 177 (30\%) died with/on disease. 117 (20\%) DLBCL had mitotic counts $>20 /$ sqmm and $31(5 \%)$ had starry sky pattern. The mean proliferation fraction was $74 \%$ (range 30-95\%) and the mean expression of myc was $47 \%$ (range $0-100 \%$ ). 276 (48 \%) cases were ABC according to the Visco's algorithm and 219 according to GEP, whereas 305 (52\%) and 236 were GCB, respectively. C-MYC gene abnormalities were detected in $41 / 453$ evaluable cases $(9 \%)$, including 2 amplifications ( $1 \mathrm{ABC}, 1$ GCB), 4 rearrangements, detectable only with the DFP (2 $\mathrm{ABC}, 2 \mathrm{GCB}), 15$ rearrangements with non-IGH partners, detectable only with the BAP ( $3 \mathrm{ABC}, 12 \mathrm{GCB}$ ) and 20 rearrangements, detectable with both the BAP and DFP (7 $\mathrm{ABC}, 13 \mathrm{GCB} ; p=0.024$ for the differences between $\mathrm{ABC}$ and GCB). There was no association between C-MYC abnormalities and lymphoma proliferative, mitotic or apoptotic activity. C-MYC aberrations weakly correlated with myc protein expression $(\mathrm{Rho}=0.132, p=0.016)$. Cases with $\mathrm{C}$ MYC gene abnormalities were more commonly primary treatment resistant to R-CHOP since 9/29 (31\%) patients with progressive disease and 5/19 (25\%) patients with stable disease had C-MYC abnormalities, compared with 27/405 (7 \%) patients, who responded to R-CHOP ( $p=0.000006$ ). Patients with C-MYC aberrations also relapsed more frequently and earlier compared with "wild type" patients (23/ $41,56 \%$, mean time to relapse 19 months vs $172 / 412,42 \%$, mean time 27 months, $p=0.055$ ). Presence of C-MYC aberrations detectable by BAP (9/15 dead, mean disease-specific survival (DSS) 29 months) or BAP and DFP (8/20 dead, mean DSS 57 months), but neither those detectable by DFL alone (1/3 dead, mean DSS 84 months) nor C-MYC amplifications (0/2 dead, mean DSS 85 months) were associated with poorer clinical outcome compared with "wild type" (115/402 dead, mean DSS 93 months, $p=0.005$ ). Importantly, radiotherapy seemed to abolish the prognostic effects of C-MYC aberrations since only $2 / 10$ patients with C-MYC abnormalities $(p=0.38)$, who were irradiated died on/with disease, compared to $16 / 28$ patients with $\mathrm{C}-\mathrm{MYC}$ abnormalities who were not given radiotherapy $(p=0.000006)$. In patients, who were not given radiotherapy, presence of C-MYC aberrations was an IPI-independent prognostic factor for DSS $(p=0.00004$, RR $1.7,95 \%$ CI $1.3-2.2$, for IPI $p=0.0002$, RR $1.4,95 \%$ CI $1.2-1.6)$.

Conclusions: C-MYC aberrations detected by BAP add prognostic information for individual DLBCL risk estimation. DLBCL patients with C-MYC aberrations may benefit from adjuvant radiotherapy.

\section{LYS071}

\section{Characterization of a series of double and triple hit lymphomas}

Mar Garcia; Anna Puiggros; Concepción FernándezRodríguez; Marta Salido; Antonio Salar; Blanca SánchezGonzález; Jose Luis Alvarez; Ariadna Torner; Ana Ferrer; Beatriz Bellosillo; Blanca Espinet; Sergi Serrano

Hospital del Mar, Barcelona, Spain

Background: B-cell lymphomas commonly exhibit single chromosomal translocations. Cases with concurrent gene rearrangements are rare and are characterized by a highly aggressive clinical behaviour, complex karyotypes and a broad morphologic and immunophenotypic spectrum. For these reasons most of the cases fall into the category of Bcell lymphoma, unclassifiable with features intermediate between diffuse large B-cell lymphoma (DLBCL) and Burkitt lymphoma (BL). In the literature, the most frequent genes that are found rearranged in these "double-hit"(DH) or "triple-hit" (TH) lymphomas are MYC and BCL2.

Aim of the study: To describe a series of $\mathrm{DH}$ and $\mathrm{TH}$ lymphomas identified in our institution between 2005 and 2012 from the morphological, immunophenotypical, molecular and clinical point of view.

Materials and methods: Twenty-four cases showing at least two translocations in either BCL2, BCL6 and/or MYC oncogenes were included in the study: 17 DLBCL, 2 follicular lymphoma (FL) grade 3A, $1 \mathrm{FL}$ grade 3B, $1 \mathrm{FL}$ grade 3B plus DLBCL, $2 \mathrm{BL}$, and $1 \mathrm{~B}$-cell lymphoma unclassifiable according to the 2008 WHO classification. For diagnostic purposes, inmunohistochemical stains, including CD20, CD3, CD10, Bc16, MUM1, Bcl2 and Ki67 had been tested previously in all cases included. The presence of mutations in the EZH2 gene were analyzed by Sanger sequencing in 21 cases. Clinical data were obtained from medical records.

Results: Of the 24 cases, 15 were males and 9 were females with a median age of 72 (range 53-94). Patients were treated with standard chemotherapy regimens. Adequate follow-up 
was available for 16 patients; 10 were exitus. The median time of follow-up was of 7.5 months (range 0-102), and the median overall survival was 5.5 months (range $0-15$ ). We identified 5 patients with antecedent/concurrent lymphoma. All but one cases (23/24) had a germinal center B-cell (GCB) phenotype. Bcl2 was expressed in 21/24 cases. Ki67 proliferation index was variable, from 50 to $100 \%$. Our series consisted of $4 \mathrm{TH}$ cases and $20 \mathrm{DH}$. The BCL2 gene was rearranged, in 19/24 cases, and MYC in 18/24 samples. BCL6 gene rearrangements occurred in 15/24 cases. In DH cases, we did not find a predominance of any specific combination (BCL2+MYC (9), BCL6+MYC (5) and BCL2+BCL6 (6)). No association was found with any of these combinations and a worse outcome, although MYC was altered in all fatal cases ( 9 translocations and 1 amplification). For the patients who died of the disease (10), 3 were TH, 3 had a combination of BCL2 and MYC rearrangements, 3 BCL6 and MYC and 1 BCL2 and BCL6. EZH2 was assessed in 21 cases of the total, and 6 cases were positive for the Y641 mutation (28,6\%).

Conclusion: B-cell neoplasms with concurrent BCL2, BCL6 and/or MYC rearrangements are clinically aggressive, irrespective of their morphological appearance, and with a germinal center B-cell immunophenotype. In our series, EZH2 gene mutations are frequent in DH or TH lymphomas; MYC, BCL2 and BCL6 are similarly rearranged, although MYC rearrangements seem to be associated with a worse outcome.

\section{LYS072}

Diffuse large B-cell lymphomas in HCV patients include a subset of cases progressed from low grade marginal zone lymphoma; clinico-pathological characterization of 51 cases from a single institution

Marco Lucioni ${ }^{1}$; Marta Nicola ${ }^{2}$; Manuel Gotti ${ }^{3}$; Roberta Riboni $^{1}$; Maria Rosa Arra ${ }^{1}$; Elena Dallera ${ }^{1}$; Silvana Molo ${ }^{4}$; Sara Rattotti ${ }^{4}$; Emanuela Boveri ${ }^{1}$; Giacomo Fiandrino ${ }^{4}$; Aldo Maffi ${ }^{4}$; Gianluca Gaidano ${ }^{4}$; Luca Arcaini ${ }^{4}$; Marco Paulli ${ }^{4}$

(1) Anatomic Pathology, Foundation IRCCS Policlinico San Matteo, Pavia (2) Anatomic Pathology, University of Pavia (3) Department of Haematology (4) Anatomic Pathology Section, University of Pavia, Italy

Background: Hepatitis $\mathrm{C}$ virus (HCV) infection has been linked to increased incidence of lymphoid neoplasms, marginal zone B-cell lymphoma (MZL) and diffuse large B-cell lymphomas (DLBCL) being the most frequent subtypes.

While HCV-related MZLs have been extensively characterized displaying distinctive clinicopathologic and molecular features, little is known about clinico-biologic features of DLBCLs in the setting of HCV chronic infection.

Methods: We selected 51 patients affected by de novo DLBCL from a series of HCV-related lymphomas, collected at a single institution. Diagnosis was established according to the 2008 WHO Classification. The histogenetic profile was determined by immunohistochemical markers including CD10, Bc16, MUM1. MALT1 and BCL2 rearrangements were investigated by means of FISH.

Results: In 31/51 (61 \%) cases lymphoma presented at extranodal sites with frequent involvement of spleen, liver and MALT sites. Centroblastic variant was the most frequent histologic subtype (78 \%). Most cases (53\%) showed a non germinal center histogenetic profile according to Hans' algorithm. In 11/51 cases (22\%), a residual small B-cell proliferation was still detectable. In 9/11 cases this cytologically low grade component was consistent with marginal zone (MZ) derived cells; a follicular appearance characterized the remaining two cases. BCL2 rearrangements were never detected; MALT1 was rearranged in 6/9 (67\%) cases with a MZ component. At the last follow-up 35/51 (69\%) patients were alive, 24 of them without evidence of disease; 16/51 (31\%) died of disease. Statistical analysis failed to demonstrate any significant prognostical factor.

Conclusion: Our series confirms that HCV-related DLBCLs are clinically, pathologically and histogenetically heterogeneous. They are likely to involve extranodal sites, including spleen and MALT tissues. Moreover our findings suggest that HCV-related DLBCLs include at least a subset of cases which might represent the final progression of a previously underlying indolent B-cell lymphoma, mostly of MZ origin and exhibiting MALT1 rearrangement.

\section{LYS073}

Evaluation of pathology review of aggressive lymphomas in the GELA/LYSA clinical trials

Thierry Jo Molina ${ }^{1}$; Josette Briere ${ }^{2}$; Tony Petrella ${ }^{3}$; Bettina Fabiani $^{4}$; Danielle Canioni ${ }^{5}$; Jean-François Emile ${ }^{6}$; Gilles Salles $^{7}$; Marie Parrens ${ }^{8}$; Corinne Haioun ${ }^{9}$; Jacques Bosq ${ }^{10}$; Hervé Tilly ${ }^{11}$; Peggy Dartigues ${ }^{10}$; Felix Reyes ${ }^{9}$; Christiane. Copie-Bergman ${ }^{9}$; Christian Gisselbrecht ${ }^{2}$; Catherine Guettier $^{10}$; Marie-Françoise d'Agay ${ }^{12}$; Bertrand Coiffier ${ }^{13}$; Jacques Diebold $^{1}$; Philippe Gaulard ${ }^{14}$

(1) AP-HP, Hôpitaux Universitaires Paris Centre, Paris (2) APHP, Saint Louis; University Paris Diderot (3) CHU Dijon (4) APHP HOPITAL SAINT ANTOINE (5) Necker Enfants Malades Hospital (6) AP-HP, Hôtel-Dieu, Paris (7) CHU Lyon-sud/HCL (8) CHU Bordeaux (9) CHU Henri Mondor (10) Institut Gustave Roussy (11) INSERM, U918, Centre Henri Becquerel (12) AP-HP, Hôpital Saint-Louis, 
Department of Pathology, (13) CHU, Lyon (14) Département de Pathologie, Hopital Henri Mondor

Background: The accurate diagnosis of lymphomas remains a challenging area for histopathologists.

Aim of the study: To evaluate for quality assurance the percentage of discordances between the initial diagnosis and the pathology panel review diagnosis in patients enrolled in GELA clinical trials.

Materials and methods: A retrospective analysis of the histological review of 8745 cases of patients included in lymphomas trials from the LNH87 (1923 pts), LNH93 (3872 pts), LNH98 (1457 pts) and LNH03 (1493 pts) programs of the GELA was performed. These trials included aggressive lymphomas (Working Formulation and/or Kiel defined) until the LNH93 program, then diffuse large B-cell lymphomas (DLBCL) and in some trials Burkitt and Anaplastic Peripheral T Cell Lymphoma in the trials after 1998. Discordances were considered when the final panel diagnostic was not belonging to the histological inclusion criteria.

Results: Discordances were $10.1 \%, 8.4 \%, 5.5 \%$ and $3.9 \%$ in the LNH87, LNH93, LNH98 and LNH03 programs, respectively. Reactive lesions representing $0,68 \%$ in the LNH87 and $0,52 \%$ in the LNH93 program (infectious mononucleosis, Kikuchi lymphadenitis) were absent in the LNH03 program. Follicular lymphoma has always been the first discordant diagnosis among all the trials representing $3.43 \%, 2.22 \%, 2 \%$ and $1.41 \%$ of all the discordances in the LNH87, LNH93, LNH98 and LNH03 programs, respectively. The percentage of all the discordant lymphoma subtypes decreased progressively within years. In the last program, the top five discordant diagnoses were; follicular lymphoma grades 1-2, mantle cell lymphoma, especially the aggressive variants, nodular lymphocyte predominant Hodgkin Lymphoma, Burkitt, lymphocytic lymphoma.

Conclusions: Our retrospective study demonstrates significant decrease in the number of major discordances within the years and no reactive diseases included in the last DLBCL trials. This improvement in the level of accuracy is probably linked to an educational part of final diagnoses sent by the GELA pathologists and to a good organization of lymphoma diagnostic management involving patients included in GELA trials.

\section{LYS074}

\section{Molecular Inversion Probe analysis Reveals Distinct Copy Number Alteration Involving Chromosome 9q24.1 in EBV-positive Diffuse Large B cell Lymphoma}

\author{
Sanghui Park ${ }^{1}$; Young Hyeh Ko ${ }^{2}$
}

(1) Gil Medical Center (2) Samsung Medical Center, Korea

Background: Diffuse large B-cell lymphoma (DLBCL) is the most common form of malignant lymphoma, accounting for $30-40 \%$ of all newly diagnosed lymphomas. Epstein Barr virus-positive diffuse large B-cell lymphoma (EBVpositive DLBCL) of the elderly is a new entity considered in the 2008 WHO classification of lymphoid malignancies and very aggressive disease with a short survival. Until now, few studies on genomic profile of EBV-positive DLBCL are available because of rare cases and difficulty to obtain fresh tumor tissue. Molecular inversion probe (MIP) technology can be successfully applied to obtain high quality copy number and genotype data with whole genome coverage from fresh tumor samples as well as formalin fixed paraffin embedded tissue.

Aim of the study: Our study attempts to investigate copy number alteration and genotype of EBV-positive DLBCL using MIP.

Materials and methods: Genomic DNA was extracted from formalin fixed paraffin embedded tissue of 17 cases of EBV-positive DLBCL. As a control, 20 cases of EBVnegative DLBCL and 11 cases of normal lymph node were used. Genomic DNA was mixed with the MIP probes (Affymetrix) with subsequent reaction of annealing, gapfill ligation, amplification, and hybridization to oligonucleotide chip array. The data was analyzed with Nexus copy number analytics Version 5.1 Software (Biodiscovery), using algorithm SNP-FASST2 with sensitivity $p$ value $<0.05$

Results: The most common DNA copy number aberrations in DLBCL regardless of EBV positivity involved chromosome 6q16.1-q21, 6q22.1-q22.2, 6q25.1, and 6q25.1-q25.2 as well as gains at 7q22.1. EBV-positive DLBCL revealed recurrent copy number gains at 1q23.3 (41\%), 2q13 (23.5\%), 3q26.31 (23.5\%), 9p24.1 (35.3\%), 12q24.1 $(23.5 \%)$ and $14 \mathrm{q} 11.2(29.4 \%)$. By enriched functional annotation terms by DAVID, genes located in those regions were mostly related to immune response, cytokine, regulation of N/F-kappa B, and apoptosis signaling.

Compared with the normal lymph node and EBV negative DLBCL, distinct change for EBV-positive DLBCL was gain of 9p24.1 (41.18 \%) which contains PD-L2 and PD-L1. These molecules play a function in the host immunity and a strong inhibitor of autologous T-cell activation.

Conclusion: We have shown the distinct copy number alteration in EBV-positive DLBCL. Further study for candidate genes at 9p24.1 would expand our knowledge on the biology of EBV-associated malignant lymphoma.

\section{LYS075 - Primary central nervous system lymphomas (PCNSL): Histological spectrum, an institutional experience} Bharti Ramnani $^{1}$; Sridhar Epari ${ }^{1}$; Sumeet Gujral ${ }^{1}$; Tanuja Shet ${ }^{1}$ 


\section{(1) Tata Memorial Hospital, Mumbai}

Background: Primary central nervous system lymphoma (PCNSL) is an uncommon extranodal non-Hodgkin lymphoma. Its incidence has been reported increasing during the last 3 decades, both in immunocompromised and immunocompetent patients. Diffuse large B-cell lymphomas constitute most PCNSLs, whereas T-cell, low-grade, anaplastic, and Hodgkin lymphomas are rarely encountered.

Materials and methods: This retrospective study included all PCNSLs seen at our institution in the last 9 years, from 2003 to 2011.

Results and conclusions: A total of 97 cases of PCNSLs were retrieved from the archival files and reviewed for histological features. The mean age was 52.8 years and $60 \%$ were found in males. Out of 97 cases, 92 cases were of B-cell type and 5 cases were of T cell type. The B-cell lymphomas included diffuse large B cell type (86 cases, $88.6 \%$ ), Burkitt lymphoma (2 cases, $2.06 \%$ ), B-cell unclassifiable (1 case, $1.03 \%$ ), lymphomatoid granulomatosis ( 2 cases, $2.06 \%$ ), and follicular lymphoma (1 case, $1.03 \%$ ). The T-cell lymphomas included anaplastic large cell lymphoma ( 2 cases, $2.06 \%$ ), peripheral T-cell lymphoma (1 case, $1.03 \%$ ) and T-cell unclassifiable (2 cases, $2.06 \%$ ). The diffuse large B-cell lymphomas were categorized into germinal center and post germinal center subtypes based on immunohistochemical reactivity of CD10, BCL2, BCL6 and MUM1. CD10 and/or BCL6 positivity and negativity for BCL2 and MUM1/IRF4 were considered characteristic of germinal center subtype and the opposite expression of post germinal center subtype.

\section{LYS076}

Analysis of MYC/8q24, BCL2/18q21 and BCL6/3q27 in diffuse large B-cell lymphoma: Correlation between FISH and immunohistochemical expression

Paloma Martín ${ }^{1}$; Diego Garcia ${ }^{1}$; Yolanda Vicente ${ }^{1}$; Carmen Bellas $^{1}$

(1) Hospital Puerta de Hierro-Majadahonda

Background: Diffuse large B-cell lymphoma (DLBCL) accounts for $30-40 \%$ of all adult non-Hodgkin's lymphomas and is an aggressive disease with marked biologic heterogeneity. Recurrent chromosomal translocations of MYC, BCL2 and BCL6 have been reported although the genetic abnormalities underlying DLBCL remain poorly understood.

Aim of the study: To analyze the status of BCL2, BCL6 and MYC by FISH and immunohistochemistry in a series of DLBCL and to correlate MYC protein expression with genetic alteration.
Materials and methods: Paraffin-embedded tumour samples of 64 patients were analyzed by immunohistochemistry for CD10, BCL2, BCL6, C-MYC, MUM1, Ki-67, GCET1, and FOXP1. Tissue microarrays (TMA) containing two representative $1-\mathrm{mm}$ cores of routinely processed tissues were prepared. Hans' and Choi's algorithm were done to classify as GC or non-GC subtype. Break Apart FISH probes for cMYC/8q24, BCL2/18q21, BCL6/3q27 were used for detecting translocations.

Results: The 64 cases were assessable for Hans' classification: 30 were classified as GC subtype and 34 as non-GC subtype. Choi's classification could be done in 62 cases: 29 were classified as GC subtype, and 33 as non-GC subtype. BCL2/18q21 gene alteration was observed in 27 samples (42\%): 6 cases translocated and amplified, 16 cases amplified and 5 cases only translocated. BCL6/3q27 gene alteration was observed in 29 samples $(45 \%)$ : 5 cases translocated and amplified, 18 cases amplified and 6 cases only translocated. c-MYC/8q24 gene alteration was observed in 15 samples $(23 \%): 3$ cases translocated and amplified, 8 cases amplified and 4 cases only translocated. One case showed BCL2, BCL6 and c-MYC rearrangement, and two cases BCL2 and c-MYC rearrangement. Protein expression of BCL2, BCL6, C-MYC and FOXP1 was detected in $75 \%, 56 \%, 23 \%$ and $40 \%$ respectively. A significant association has been found between c-MYC rearrangement and protein expression and between 18q21 alteration (amplification and translocation) and BCL2 expression. FOXP1 is associated $(p=0.003)$ with C-MYC expression. Different gene rearrangements were not correlated with GC or non-GC immunophenotype. Non-GC phenotype was significantly associated with shorter overall survival.

Conclusions: 1) In our study c-MYC, BCL2, or BCL6 translocations are not associated with $\mathrm{GC}$ or non-GC immunophenotype. 2) MYC protein expression is associated with c-MYC gene alteration (translocation and amplification). 3) A significant association has been found between C-MYC protein expression and FOXP1 expression.

\section{LYS077}

MicroRNA profiling of primary cutaneous large B-cell lymphoma

Koens, L ${ }^{1}$; Qin, $\mathrm{Y}^{1}$; Leung, W.Y ${ }^{1}$; Hageman, $\mathrm{L}^{1}$; Corver, W.E ${ }^{1}$; Jansen, P.M ${ }^{1}$; Willemze, R ${ }^{1}$; Vermeer, M.H ${ }^{1}$; Tensen, C.P ${ }^{1}$

(1) Leiden University Medical Center, The Netherlands

Background: MicroRNAs are small non-coding RNAs that regulate gene expression at a posttranscriptional level. Their 
aberrant expression is known to be pathogenetically involved in many diseases. Although a role of microRNAs in nodal diffuse large B-cell lymphomas (DLBCLs) is generally recognized, the presence and function of microRNAs in primary cutaneous large B-cell lymphomas (PCLBCLs) are not yet described. The two types of PCLBCL, primary cutaneous diffuse large B-cell lymphoma, leg type (PCBCLLT) and primary cutaneous follicle center lymphoma (PCFCL) are characterized by an activated B-cell (ABC) genotype and a germinal center B-cell (GCB) genotype, respectively.

Aim of the study: Establishing microRNA profiles of PCFCL and PCBCL-LT and discovering specific microRNAs that show differential expression between these two tumor types.

Materials and methods: By performing high-throughput next generation sequencing analysis, the microRNA profiles of frozen tumor biopsies from 11 cases of PCBCL-LT and 6 cases of PCFCL were established. Quantitative analysis for differential expression of microRNAs between the two tumor types was performed. For a selection of these microRNAs real-time qPCR on both internal and external validation groups was performed, along with a set of microRNAs known to be involved in the pathogenesis of nodal DLBCLs.

Results: The sequence data showed 16 microRNAs that were differentially expressed between PCBCL-LT and PCFCL $(p<0.05$, Benjamini-Hochberg corrected). However, 5 microRNAs commonly described to be differentially expressed between GCB and ABC type nodal DLBCL were not amongst them. Higher expression of miR-9, miR-31, miR-129-2 and miR-214 in PCFCL as compared to PCBCL-LT was confirmed by qPCR.

Conclusions: This approach allowed the identification of a microRNA signature discriminating PCFCL from PCBCLLT. These results show substantial differences as compared to the previously described signature(s) discriminating $\mathrm{GCB}$ from $\mathrm{ABC}$ type nodal DLBCLs.

\section{LYS078}

Epstein-Barr virus-positive classic Hodgkin lymphoma in Iraqi children: an immunohistochemical and molecular study

Arianna Di Napoli ${ }^{1}$; Mazin F. Al-Jadiri ${ }^{1}$; Caterina Talerico ${ }^{1}$; Enrico Duranti ${ }^{1}$; Emanuela Pilozzi ${ }^{1}$; Pankaj Trivedi ${ }^{1}$; Eleni Anastasiadu $^{1}$; Adel R. Alsaadawi ${ }^{1}$; Amir F. Al-Darraji ${ }^{1}$; Salma A. Al-Hadad ${ }^{1}$; Anna Maria Testi ${ }^{1}$; Luigi Ruco ${ }^{1}$; Stefania Uccini ${ }^{1}$

(1) Sant'Andrea Hospital, La Sapienza University, Rome (2) Children Welfare Teaching Hospital, Baghdad College of Medicine
Background: Classic Hodgkin lymphoma (cHL) in children under 14 years of age is often associated with EBV infection, and is more commonly observed in developing countries. It is not yet clear if EBV-positive cHL of childhood and EBV-negative cHL of young adults are different diseases or are different forms of the same disease.

Materials and methods: Fifty cases of Hodgkin lymphoma affecting Iraqi children under 14 years of age were investigated on biopsies for immunophenotypic profile, EBVLMP-1 gene polymorphism and, TCR $\gamma$ and IGH genes rearrangements.

Results: EBV infection of H/RS cells was demonstrated in 39 of 48 cases $(81 \%)$. It was more common in Mixed Cellularity (MC) histology and in younger children. Increasing the age, there was a decrease of EBV-positive cases (from $100 \%$ to $75 \%$ ), and an increase in Nodular Sclerosis (NS) cases (from $12 \%$ to $38 \%$ ). Wild type LMP-1 gene was found in $68 \%$, whereas the LMP-1 deleted variant of EBV was detected in $32 \%$; no significant association was found between the presence of LMP-1 deletion and any of the clinico-pathological features of the disease. Oligoclonal/monoclonal TCR $\gamma$ rearrangements were present in 23/39 EBV-positive Iraqi cases, in 5/5 EBVnegative Iraqi cases, and in 0/9 EBV-negative Italian cases.

Conclusions: Our results demonstrate that $\mathrm{cHL}$ affecting Iraqi children is undistinguishable from that occurring in western countries except for the high incidence of oligoclonal/monoclonal TCR $\gamma$ rearrangements in $\mathrm{cHL}$ of childhood even in EBV-negative cases. It is speculated that this finding might be expression of an antigen-driven immune response, perhaps sustained by a previous EBV infection. The possibility is discussed that in cHL of childhood (under 14 years of age) EBV-positive and EBV-negative cases are different forms of the same disease, and that there is a gradual, age-dependent, transition from EBV-positive MC to EBV-negative NS.

\section{LYS079}

MicroRNA expression profile identifies a distinct molecular signature between $M Y C$ translocation positive and negative Burkitt lymphoma cases

Anna Onnis ${ }^{1}$; Federica Morettini ${ }^{1}$; Fabio Fuligni ${ }^{2}$; Giulia de Falco $^{1}$; Cristiana Bella ${ }^{1}$; Maura Rossi ${ }^{2}$; Maria Rosaria Sapienza $^{2}$; Emily A Rogena ${ }^{3}$; Pierpaolo Piccaluga ${ }^{2}$; Lorenzo Leoncini $^{1}$

(1) Department of Human Pathology and Oncology, University of Siena, Siena, Italy (2) Haemolymphopathology Unit - S. Orsola-Malpighi Hospital - University of Bologna - Italy (3) Department of Human Pathology, University of Nairobi

Background: The MYC oncogenic transcription factor is pathologically activated in many human malignancies. A 
paradigm for $M Y C$ deregulation is offered by Burkitt lymphoma (BL), where chromosomal translocations that join $M Y C$ with immunoglobulin heavy- or light-chain loci are the crucial initiating oncogenic events. However, about $10 \%$ of typical BLs lack an identifiable $M Y C$ rearrangement, although no significant difference of c-MYC expression among these cases has been observed. Additional mechanisms alternative to translocations, which could result in c-MYC dysregulation, were proposed, including microRNA (miRNA) deregulation.

Aim of the study: The aim of this project was to identify possible differences in gene and miRNA expression in BL cases with or without MYC translocation. BL were also used as a model to investigate the existence of alternative molecular mechanisms other than chromosomal translocations leading to MYC deregulation.

Materials and methods: Twenty formalin-fixed and paraffinembedded BL specimens (10 MYC translocation-positive and 10 MYC translocation-negative) were used for this study. For miRNA and gene expression profile (GEP), the samples were hybridized on the miRCURYTM LNA Array and the Illumina DASL whole genome Assay, respectively. Validation by qPCR and functional assays were performed for selected targets.

Results: We identified four miRNAs differentially expressed between the two groups. The relationship of the expression patterns of the differentially regulated miRNAs and their targets was then investigated by GEP. Interestingly, these miRNAs significantly impacted GEP, controlling relevant biological processes, such as the angiogenesis, apoptosis and cell proliferation, according to Gene Ontology categories. Among others, we found hsa-mir-34b, which can modulate MYC expression in vivo.

Conclusions: We showed for the first time that MYC translocation-positive and negative BLs differs for both a miRNA and a gene signature. The identification of specific altered miRNAs may represent an alternative molecular mechanism leading to c-MYC over-expression in the absence of other genetic alterations.

LYS080 - Prognostic value of p53 and p16 expressions in nodal diffuse large B-cell lymphoma: correlations with clinicopathologic parameters and survival

Ozlem Canoz ${ }^{1}$

(1) Erciyes University Pathology Department Kayseri, Turkey

Background: Diffuse large B-cell lymphomas (DLBL) represent a diverse spectrum of lymphoid neoplasms with variable clinical, histopathological, immunophenotypic and cytogenetic features. In DLBL, besides the clinical parameters, it has become more important gene expressions such as tumor-supressor gene proteins, oncogen proteins in determining the prognosis, biological behavior of the tumor and new treatment regimens.

Aim of the study: In order to define the prognostic impact of p16 and p53 expressions in DLBL, we retrospectively investigated 40 patients in whom biopsy specimens were available for further pathologic examinations.

Materials and methods: A total of 40 patients with nodal DLBCL diagnosed between 2005 and 2010 in the Department of Pathology, Medical Faculty Erciyes University were included in the study. Immunohistochemical analysis was performed using a panel of monoclonal antibodies for CD10, BCL6, MUM1, Ki-67, p53, p16, and evaluated by light microscopy on slides. With CD10, BCL6 and MUM1 DLBL were categorized into two subgroups: germinal center B-cell-like (GCB) and non-germinal center B-cell-like (nonGCB) type, using algorithm proposed by Hans et al. A cut of value of $10 \%$ positive cells was used for p16 staining.

Results: The median age of the patients was $60.50 \pm 16.92$ years old. The median follow-up period was $16.58 \pm 13.68$ months. The relationship between clinical and laboratory parameters such as, age, gender, performance status, clinical stage, presence of B-symptoms, bone marrow involvement, International Prognostic Index (IPI) score, lactate dehydrogenase (LDH) level, extranodal extension, relapse, C-reaktive protein (CRP), sedimentation rate, number of leukocytes and overall survival was evaluated. Additionally, the expression of p16, p53 and Ki-67 expression was analyzed. In our study, there was statistically significant relation between p16 expression and stage $(p<0.05)$, and no statistically significant correlation between the p53 and p16 expressions, Ki-67 proliferation index and both clinical parameters, cell origin and overall survival $(p>0.05)$.

Conclusions: Since there was no difference between GCB and non-GCB type and oncogene expressions, we will focused in only GCB type group to evaluate intermediate-high risk status for this group.

\section{LYS081}

SOXC transcription factors in mantle cell lymphoma promoter methylation is not the main underlying mechanism for differences in SOX11 expression

Birgitta Sander ${ }^{1}$; Agata M. Wasik ${ }^{1}$; Martin Lord ${ }^{1}$; Xiao Wang $^{1}$; Fang Zong ${ }^{1}$; Patrik Andersson ${ }^{1}$; Eva Kimby ${ }^{1}$; Birger Christensson ${ }^{1}$; Mohsen Karimi ${ }^{1}$

(1) Karolinska University Hospital Huddinge, Karolinska Institute

Background: The related transcription factors SOX4, SOX11 and SOX12 (the SOXC group of transcription factors) compete for the same target genes. SOX11 is 
expressed in most mantle cell lymphomas (MCL), but a small subset is, like normal lymphocytes, SOX11 negative. SOX11 expression in MCL, as compared to other lymphomas and normal lymphocytes, has been suggested to be regulated by histone modifications and/or promoter methylation

Aim of the study: To investigate the expression of SOXC genes in MCL and non-malignant lymphocytes, and to investigate whether SOX11 expression in MCL, as compared to non-malignant lymphocytes, is regulated by promoter methylation.

Materials and methods: SOXC expression was investigated by QPCR in 13 primary MCL and 4 MCL cells lines (Granta519, JeKo, Rec1 and JVM2), non-malignant lymphoid tissue from lymph node and spleen and in purified B cells from tonsil. SOX11 expression was also investigated by IHC and WB. Promoter methylation was investigated by McrBCbased methylation assay and by bisulfite sequencing. MCL cell lines were treated with 5-azacytidine (5-AZA) and SOXC expression was quantified during 6 days of treatment.

Results: Compared to non-malignant B-lymphocytes, SOX11 mRNA was highly expressed in $11 / 13$ primary MCL and 3/4 MCL cell lines. SOX4 mRNA was less expressed in 10/13 MCL cases than in non-malignant $\mathrm{B}$ lymphocytes. SOX12 mRNA was $2-17$ fold higher in MCL than in non-malignant B-lymphocytes. Two MCL cases expressed all three SOXC transcription factors. The two SOX11 negative MCL cases expressed SOX12. The SOX11 promoter region was hypomethylated in SOX11 positive and one SOX11 negative MCL, while the other SOX11 negative MCL case showed some degree of methylation. Importantly, also non-malignant B lymphocytes and lymphoid tissue had hypomethylated SOX11 promoter region.

5-AZA treatment did not induce SOX11 expression in SOX11 positive or SOX11 negative MCL cell lines. Instead, SOX11 mRNA and protein decreased in Granta519 and Rec1 SOX11 in response to 5-AZA. In the SOX11 negative MCL cell line JVM2, treatment with 5-AZA induced expression of SOX4 mRNA but not SOX11 or SOX12.

Conclusions: The SOX11 promoter region is hypomethylated both in SOX11 positive and SOX11 negative non-malignant lymphoid tissue and is heterogenously methylated in SOX11 negative primary MCL cases. The expression of SOX11 in MCL is therefore regulated by other mechanisms than promoter methylation as suggested by Vegliante et al (PLoS One.6(6): e21382). This result is further supported by the inability to induce SOX11 expression by 5-AZA. SOX12 is overexpressed in SOX11 positive and SOX11 negative MCL compared to non-malignant lymphocytes. SOX4 is generally less expressed in MCL than in non-malignant lymphocytes but could be induced by 5 -AZA in the SOX11 negative MCL cell line JVM2. In primary MCL, SOX4 and SOX11 were coexpressed in 2/13 cases, both of these cases were blastoid variants. Since members of the SOXC group of transcription factors have partly overlapping functions, the differential expression in MCL may have important biological functions.

\section{LYS082}

\section{In vitro cultivation of primary follicular lymphomas}

Annette M. Staiger ${ }^{1}$; Heike Horn ${ }^{1}$; Michael A. Dengler ${ }^{1}$; Heiko van der Kuip ${ }^{1}$; Walter E. Aulitzky ${ }^{1}$; Andreas Rosenwald $^{1}$; German $\mathrm{Ott}^{2}$

(1) Institut für klinische Pharmakologie (2) Department of Clinical Pathology, Robert-Bosch-Krankenhaus, and Dr. Margarete Fischer-Bosch Institut

Background: Follicular lymphomas (FL) represent the most frequent type of malignant lymphomas in western countries. The biological features and the clinical course of FL are highly variable. Many attempts have been made to discover possible mechanisms explaining this variation. However, no reliable in vitro model of FL exists.

Aim of the study: Therefore, we established an in vitro system of primary FL, allowing the investigation of potential tumor-relevant features.

Materials and methods: Primary lymph nodes from 11 patients with FL were investigated. Either non-separated cell fraction, negative or positive selected B-cells (CD3, CD14, CD16 or CD19) were used. Flow cytometry was performed for validation of the cell population (CD19, CD20, CD10), measuring cell viability (AnnexinV) and cell cycle distribution (Propidium Iodide). Different culture conditions have been tested, using either separated or non-separated cells, with or without soluble CD40-Ligand or with or without a feederlayer of stromal fibroblast cell line (HS-5) and tumor associated fibroblasts isolated from primary FL. To analyze fibroblast-mediated co-culture effects, cells were treated with cytotoxic agents (ABT-737, Bortezomib).

Results: The non-separated FL cells showed the highest viability $(85 \%, 80 \%$ and $70 \%$ after 24,48 and $72 \mathrm{~h}$, respectively). There was no advantage of additive culture conditions. While there was no effect on tumor cell viability when cocultured with either HS-5, autologous or heterologous tumorassociated fibroblasts, tumor cells were protected from induction of cell death by stromal cells when treated with ABT-737 or Bortezomib. Furthermore, there was a loss of CD19 and CD10 during cell death. Therefore B-cell measurements have been accomplished with CD20-staining. Comparing cell cycle analysis of viable cells with FFPE immunohistochemistry of Ki67, we obtained concordant results.

Conclusions: The high viability of non-separated FL cells provides a physiological in vitro system. The finding of protective mechanisms mediated by fibroblasts is of great interest for further studying cytotoxic agents in FL. 


\section{LYS083}

\section{A clinically active BTK inhibitor targets adhesion and migration in chronic lymphocytic leukemia and mantle cell lymphoma}

Steven T. Pals ${ }^{1}$; Martin F.M. de Rooij ${ }^{2}$; Annemieke Kuil ${ }^{3}$; Christian R. Geest ${ }^{4}$; Eric Eldering ${ }^{4}$; Betty Y. Chang ${ }^{5}$; Joseph J. Buggy ${ }^{5}$; Marcel Spaargaren ${ }^{6}$

(1) Academic Medical Center, Amsterdam (2) Department of Pathology, Academic Medical Center, University of Amsterdam (3) Department of Pathology, Academic Medical Center, University of Amsterdam, Amsterdam (4) Department of Immunology, Academic Medical Center, University of Amsterdam, Amsterdam (5) Pharmacyclics Inc (6) Department of Pathology, Amsterdam Medical Center, University of Amsterdam, Amsterdam

Background: Several novel small molecule drugs targeting the B-cell antigen receptor (BCR) signalosome have recently demonstrated promising efficacy in the treatment of B-cell non-Hodgkin lymphoma. Most of these agents, including the BTK inhibitor PCI-32765, display an unexpected clinical response in chronic lymphocytic leukaemia (CLL) and mantle cell lymphoma (MCL): a rapid and sustained reduction of lymphadenopathy accompanied by transient lymphocytosis, which is reversible upon temporary deprivation of the drug. We have previously demonstrated that the B-cell antigen receptor (BCR) controls integrin ?4?1-mediated adhesion of $\mathrm{B}$ cells to fibronectin and VCAM1, which is mediated by Bruton's tyrosine kinase (BTK). More recently, we have shown that also chemokines induce activation of BTK and that integrin-mediated adhesion and migration in response to CXCL12 or CXCL13, as well as in vivo homing to lymphoid organs, is impaired in BTK-deficient B cells.

Aim of the study: We hypothesized that the clinical response observed upon PCI-32765 treatment reflects attenuated lymph node and bone marrow homing and retention of the CLL and MCL cells, due to impaired BCR- or chemokine-controlled integrin-mediated adhesion or migration.

Materials and methods: The Burkitt lymphoma cell line Namalwa or primary patient CLL and MCL cells, pretreated with PCI-32765, were allowed to adhere to either fibronectin- or VCAM-1-coated surfaces in the presence of anti-IgM, PMA, or chemokines (CXCL12, CXCL13 or CCL19), or were allowed to migrate towards these chemokines in VCAM-1-coated Transwells,

Results: We found that PCI-32765 strongly inhibits BCRcontrolled signaling and integrin $\alpha 4 \beta 1$-mediated adhesion to fibronectin and VCAM-1, both in Namalwa and primary CLL cells. Furthermore, PCI-32765 also inhibits CXCL12-induced signaling and CXCL12-, CXCL13- and CCL19-induced adhesion and migration of primary CLL and MCL cells.

Conclusions: Our data indicate that inhibition of BTK by PCI-32765 overcomes BCR- and chemokine-controlled homing and retention of the malignant $\mathrm{B}$ cells in their growth- and survival-supporting microenvironment, resulting in clinically evident CLL and MCL regression.

\section{LYS084}

Frequent somatic alterations of $\mathrm{NF}-\mathrm{K} B$ regulators in ocular adnexal MALT lymphoma

Ming Wang ${ }^{1}$; Qingguo $\mathrm{Yan}^{2}$; Sarah Moody ${ }^{3}$; Yuanxue Huang ${ }^{2}$; Yingwen $\mathrm{Bi}^{2}$; Ming-Qing $\mathrm{Du}^{2}$

(1) University of Cambridge (2) Department of Pathology, University of Cambridge (3) mw525@cam.ac.uk

Background: Ocular adnexal MALT lymphoma typically lacks chromosome translocations that are associated with MALT lymphoma. The genetics of these translocation negative MALT lymphomas is largely unknown. In a recent study, we demonstrated inactivation of TNFAIP3 (also known as A20, encoding for a global NF-kB inhibitor) by somatic inactivating mutations in $28.6 \%$ of ocular adnexal MALT lymphomas. To investigate further the extent of NF-kB pathway abnormalities in ocular adnexal MALT lymphoma, we investigated somatic mutation of several other NF- $\mathrm{KB}$ regulators including MYD88, CARD11, CD79A/B and API2, as well as PRDM1 (BLIMP1), which are frequently involved in other B-cell lymphoma subtypes characterized by constitutive NF-KB activation.

Material and methods: The coding exons of the above genes were PCR-amplified and sequenced using the Sanger method. Mutations identified were excluded from germline changes by analyses of DNA samples extracted from the microdissected normal cells.

Results: MYD88 mutation was detected in 6 (5.7\%) of 105 ocular adnexal MALT lymphomas and was largely mutually exclusive from A20 inactivating mutation. Among the MYD88 mutations detected, four were missense changes (L265P in 3 cases, S243N in 1 case) and the remaining two were an in-frame deletion (delT285-T294). In vitro reporter assays showed that all the above MYD88 mutants were potent activator of both NF-kB and AP1 transcription factors. CARD11 mutation was seen in a single case, while CD79A/B, API2 and PRDM1 somatic mutation was not found in any of the cases investigated.

Conclusions: In total, $35 \%$ of ocular adnexal MALT lymphoma showed evidence of activation of NF- $\mathrm{KB}$ positive regulators and/or inactivation of $\mathrm{NF}-\mathrm{KB}$ negative regulators, suggesting an important role of constitutive NF- $\mathrm{KB}$ activation in the lymphoma pathogenesis. 


\section{LYS085}

CSF1R protein expression in reactive lymphoid tissues and lymphoma microenvironment

Juan F. Garcia ${ }^{1}$; Ana M. Martin-Moreno ${ }^{2}$; Giovanna Roncador $^{3}$; Carmen Rubio ${ }^{1}$; Scherezade Jiménez ${ }^{4}$; Jorge L. Martínez-Torrecuadrada ${ }^{4}$; Lorena Maestre ${ }^{4}$; José F. Tomás ${ }^{1}$; Mónica Estévez ${ }^{1}$; Miguel A. Piris ${ }^{5}$

(1) MD Anderson Cancer Center Madrid (2) MD Anderson Cancer Center Madrid, Pathology Department (3) Spanish National Cancer Research Center (CNIO), Biotechnology Programme (4) Spanish National Cancer Research Center (CNIO), Molecular Pathology Programme (5) Department of Hematology, Hospital Universitario Marqués de Valdecilla

Background: Tumor-associated macrophages (TAM) have been associated with survival in follicular lymphoma and classical Hodgkin lymphoma. The maturation and differentiation of tissue macrophages depend on the interactions between colony-stimulating factor 1 receptor (CSF1R) and its ligand (CSF1). Aberrant expression of CSF1R has been described in cHL-derived cell lines and tumors.

Materials and methods: We analyzed by immunohistochemistry (IHC) the distribution of CSF1R protein in FFPE samples, using a new monoclonal antibody raised against the extracellular domain of the receptor. Samples included were 24 reactive lymphoid tissues (RLT) (lymph nodes, tonsils, bone marrow, thymus, and spleen), and 187 different lymphoma types (10 B-LBL, 20 B-CLL, 18 FCL, 20 MCL, 20 DLBCL, 19 BL, 5 MALT, 8 T-LBL, 15 PCTCL, 15 AITL, 2 ALCL, and 35 cHL). We also analyzed by double IHC the distribution of CSF1R+ cells, CD163+ (M2) macrophages, FOXP3+ (Tregs), and PD1+ T-cells. Additionally, we studied the relationship between CSF1R expression and clinical outcome in an independent series of $220 \mathrm{cHL}$ patients.

Results: CSF1R + cells are abundant in the red pulp of the spleen, the T-zone and paracortical area of lymph nodes and tonsils, and the medulla of the thymus. The distribution of CSF1R+ cells did not overlap with CD163+ (M2) macrophages. Double IHC showed a striking association between CSF1R + macrophages and FOXP3+ Tregs.

The number of CSF1R+TAMs differs across different lymphoma types. Lower amount was found in B-CLL and LBL, whereas higher number was identified in the microenvironment of PTCL, AITL, and cHL. In cHL, HRS cells did not show noticeable levels of CSF1R protein. Tumors characterized by a CSF1R+/FOXP3+ enriched microenvironment showed longer survival than cases characterized by a predominant CD163+/PD1+ pattern.

Conclusions: CSF1R identifies a specific macrophage subpopulation associated with FOXP3 + Tregs in RLT and tumors. CSF1R+ cells are abundant in the microenvironment of T-cell lymphomas and subsets of cHL. In cHL, the tumors characterized by a CSF1R+/FOXP3+ background are associated with better clinical outcome.

\section{LYS086}

Hodgkin's lymphoma in the hard palate: a new diagnostic perspective

Jéssica E. Fulfaro ${ }^{1}$; Thais S. de Oliveira $^{1}$; Rafael D. Gaiolla $^{1}$; Cristiano C. Oliveira ${ }^{1}$; Maria Ap. C. Domingues ${ }^{1}$

\section{(1) São Paulo State University (UNESP), Brazil}

Background: Hodgkin's lymphoma (HL) is a malignant lymphoid neoplasia that presents nodal manifestations and extranodal manifestations are rare. It is characterized by the presence of binucleated cells called Reed-Sternberg (RS), uninucleated cells called Hodgkin $(\mathrm{H})$ and infiltration of lymphocytes, plasma cells and eosinophils. Hard palate involvement as a primary site is unusual, with only eight cases of primary LH in the oral mucosa previously described in the English language literature, of which only 3 were located in the palate. Here we report an unusual manifestation of $\mathrm{LH}$ affecting the hard palate, with clinical aspects similar to mucocutaneous infectious diseases.

Results: A 43-year-old female patient complained of odynophagia for 6 months. During the physical examination, an ulcer was observed in the hard palate measuring $2.0 \mathrm{~cm}$ and presenting communication with the nasal cavity. Initial hypotheses included leishmaniasis, tuberculosis or mycosis, investigated by serological tests. An incisional biopsy of the lesion was also performed, followed by histological analysis and immunohistochemistry for CD15, CD30, CD45 and Fascin markers. Exams involving etiological agents for possible infections were all negative. Incisional biopsy revealed lymphocytic infiltration, with rare RS cells and $\mathrm{H}$, while the immunohistochemistry exams were positive for CD30 and fascin and negative for CD15 and CD45 markers, indicating a diagnosis of primary $\mathrm{HL}$ of the palate. At the time, the clinical stage was IA. Three cycles of ABVD chemotherapy were performed and no new lesions appeared.

Conclusions: Primary extranodal HL of the oral cavity suggests a new diagnostic and epidemiological perspective in medical practice. Its rarity and difficulties regarding its control could be associated with a new entity and this extends to clinical reasoning regarding the differential diagnosis of etiologies of ulcerative lesions of the palate. 


\section{LYS087}

Tyrosine phosphorylation of STAT3 in chronic lymphocytic leukemia: a mechanism involved in Richter syndrome transformation

Aline-Fardin ${ }^{1}$; Claude Capron ${ }^{1}$; Bettina Fabiani ${ }^{1}$; JeanFrançois Emile ${ }^{1}$; Paul Coppo ${ }^{1}$

(1) Department of pathology Hopital Saint-Antoine Paris, France

Background: The mechanisms involved in the transformation of chronic lymphocytic leukemia (CLL) into a more aggressive lymphoma (Richter syndrome [RS]) still remain unclear. In CLL, the signal transducer and activator of transcription 3 (STAT3) was found oncogenic and constitutively phosphorylated in serine 727 residue (Ser727), but not in tyrosine 705 residue (Tyr705), which is involved in STAT3 dimerization and nuclear translocation.

Aim of the study: We hypothesized that Tyr705 phosphorylation of STAT3 could be involved in CLL transformation into RS.

Materials and methods: STAT3 Tyr705 and Ser727 phosphorylation was studied by immunophenotyping from peripheral blood mononuclear cells or by immunohistochemistry on paraffin sections from lymph node biopsies to detect Ser727 and Tyr705 phosphorylation of STAT3 in RS (4 cases) and accelerated CLL (2 cases). STAT3 was inhibited with smallinterference RNA (shRNA) on RS cells from a patient with leukemic RS. We used shSTAT5 and an empty vector as controls. Apoptosis was studied after annexin-V staining.

Results: From 20 patients with CLL, we confirmed that STAT3 was phosphorylated in Ser727, but not in Tyr705. On the opposite, we found a constitutive Tyr705 phosphorylation in all 4 cases (100\%) of RS. In 1 patient with accelerated CLL, STAT3 Tyr705 phosphorylation was only observed in large lymphoid cells, but not in the small lymphoid component. Inhibition of STAT3 by shRNA in circulating RS cells from 1 patient was associated with increased apoptosis (13\% of apoptotic cells) when compared with STAT5 inhibition or with cells transduced with the empty vector (13\% versus $3 \%$ and $4.5 \%$, respectively).

Conclusions: We show for the first time that STAT3 Tyr705 is constitutively phosphorylated in RS, but not in CLL. STAT3 inhibition in RS cells is associated with an increased apoptosis. These results suggest that phosphorylation of STAT3 on Tyr705 in CLL could be involved in its progression into a more aggressive disease.
LYS088

\section{CD99 expression and newly diagnosed diffuse large B-cell lymphoma treated with rituximab-CHOP immunochemotherapy}

Taeeun Kim ${ }^{1}$; Sanghui Park ${ }^{2}$; Ji Yeon Kim ${ }^{3}$

(1) Gachon University Gil Medical Center (2) Ewha Womens University School of Medicine (3) Inje University Haeundae Paik Hospital

Background: Diffuse large B-cell lymphoma is the most common subtype of non- Hodgkin's lymphoma and identification of prognostic factors of DLBCL is important. Recently, CD99 expression has been documented in DLBCL but few studies have investigated the prognostic impact of CD99 in DLBCL.

Aim of the study: To evaluate prognostic value of CD99 expression in patients with DLBCL who underwent treatment with rituximab-CHOP immunochemotherapy

Materials and methods: Immunohistochemistry for CD99/ CD10/BCL-2/BCL-6/MUM-1 was performed on nodal DLBCL specimens from 70 patients. Patients were classified as either germinal center B-cell subtype (GCBS) or non-GCBS, according to the Muris algorithm.

Results: A superior two-year event-free survival (EFS) was observed in patients with GCBS, compared to those with non-GCBS $(p=0.034)$. CD99 expression (29 patients; $41.4 \%$ ) did not show deviation according to subtype and was not prognostic for survival in the entire patient population observed in CD99+ patients, compared to CD99- patients. Conversely, among patients with non-GCBS, inferior EFS and OS were reported in CD99+ patients.

Conclusions: Superior two-year EFS $(p=0.004)$ and twoyear OS $(p=0.003)$ were observed in patients with GCB/ CD99+ and non-GCB/CD99-, compared to the others, and the combination classification was found to be an independent prognostic factor.

\section{LYS089}

The ratio of the absolute lymphocyte count to the absolute monocyte count is associated with prognosis in Hodgkin's lymphoma: correlation with tumor-associated macrophages

Miji Lee ${ }^{1}$; Youngwha $\mathrm{Koh}^{1}$; Hyo Jeong Kang ${ }^{1}$; Chansik Park $^{1}$; Jooryung Huh ${ }^{1}$

(1) Asan medical center, Seoul, Korea

Background: Although most patients with classical Hodgkin's lymphoma (cHL) have a long survival duration, the current risk 
stratification is imperfect. A recent study suggested a prognostic role for the peripheral blood absolute lymphocytecount/absolute monocyte count (ALC/AMC) ratio at diagnosisin cHL. It is intriguing to investigate the significance of the ALC/AMC ratio in relation to tumor-associated macrophages (TAMs), yet another prognostic factor for $\mathrm{cHL}$.

Materials and methods: We examined the prognostic impact of the ALC, AMC, and ALC/AMC ratio in $312 \mathrm{cHL}$ patients (median age, 37 years) using receiver-operating characteristic curve analysis for optimal cutoff values, and compared these with TAM content.

Results: The median follow-up was 65 months (range, 0.1245 months). On univariate analysis, a low ALC/AMC ratio $(<2.9)$ was correlated with a poorer overall survival (OS) outcome. A subgroup analysis of patients with limitedstage disease showed that the ALC/AMC ratio was significantly correlated with the OS time. Multivariate analysis showed the ALC/AMC ratio to be an independent prognostic factor for OS outcome. A Spearman correlation test of TAM content showed a negative correlation with the ALC/AMC ratio and a positive correlation with the peripheral blood macrophage percentage.

Conclusions: This study suggests that the ALC/AMC ratio may be a simple, inexpensive, and independent prognostic factor for OS outcome in patients with $\mathrm{cHL}$ and may have a role in the stratification of $\mathrm{cHL}$ patients in addition to the International Prognostic Score and TAM content. Oncologist. 2012 May 15. [Epub ahead of print]

\section{LYS 091}

Collective review of Mantle cell Lymphoma-clinicopathologic evaluation and Sox 11, p27, CXCR4, Ki-67 expression; Korean 94 cases

Hyun-Jung Kim ${ }^{1}$; Jooryung $\mathrm{Huh}^{2}$; Yoo-Duk Choi ${ }^{3}$; Jin Man $\mathrm{Kim}^{4}$; HeeJeong Cha ${ }^{5}$; Soo Kee Min ${ }^{6}$; Dae Woon Eom ${ }^{7}$; Ji Eun $\mathrm{Kim}^{8}$; Gyeongsin Park ${ }^{9}$; Hojung Lee ${ }^{10}$; Jin Ho Paik ${ }^{11}$

(1) Inje Univ. Sanggye Paik Hospital, Seoul, Korea (2) Asan Medical Center Ulsan college of Medicine (3) Chonnam National University Medical School (4) Chungnam National University School of Medicine (5) Ulsan Univ Hospital (6) Hallym University, Hallym Sacred Heart Hospital (7) University of Ulsan College of Medicine, Gangneung Asan Hospital (8) Department of Pathology, Seoul National University Boramae Hospital (9) Seoul St. Mary's Hospital, The Catholoic University of Korea (10) Eulji University School of Medicine (11) Seoul National University Bundang Hospital

Background: Mantle cell Lymphoma (MCL) is a distinct subtype of B cell non-Hodgkin lymphoma (NHL) which shows unfavorable prognosis. Response to conventional chemotherapy is usually poor with 3-5 years of median survival time. Recently, a new diagnostic marker, Sox-11 has been introduced and its clinical significance is being discussed.

Aim of the study: This study was designed for a collective review of MCL and evaluation of p27, CXCR4, and Ki-67 expressions as well as Sox 11 on a large volume of Korean cases.

Materials and methods: Ninety-four cases of MCL with available tissue were enrolled from 11 institutes in Korea. Among them, tissue microarray containing $1 \mathrm{~mm}$ core in triplicate was used in 43 cases. Clinicopathologic features were reviewed and immunohistochemistry (IHC) for Sox-11, p27, CXCR4, and Ki-67 was performed. Cases were divided into good prognostic group, designated as complete remission without relapse during more than 3 years follow up, and the other. Results: 1) The age of the patients was arranged from 36 to 99 [mean age; 70.05]. The most prevalent in 8th decade (28), then followed by 7 th decade (26), 6th (17), 9th (12). Male patients were predominant (M: $F=71: 23)$. Sixty-four cases had nodal lymphoma, and 30 had extranodal lymphomas; most commonly gastrointestinal tract consisting of small intestine $(n=5)$, large intestine $(n=4)$ and stomach $(n=4)$. Head and neck area was the second frequent site consisting of tonsil $(n=4) /$ adenoid $(n=1)$ and nasopharynx $(n=3)$. Others are various glandular lesions (4) (parotid, lacrimal, prostate, breast-each one case), spleen (2), and skin (2). 2) Morphologically, there were 81 typical cases and 13 pleomorphic/blastoid variant, 74 diffuse and 20 nodular types $[p=0.01]$. 3) Among 94 cases, Sox-11 immunohistochemical staining showed negative in 8 cases $(8.5 \%)$ and variable expression in 86 cases $(91.5 \%)$. The expression of Cyclin D1 was negative in 10 cases. Outcomes of 8 Sox-11 negative cases are 3 cases of CR, 2 cases of PD, and 2 cases of PR including one case of F/U loss. Sox-11 negative cases were associated with a relatively better outcome than others, but but it should be noted that leukemic or indolent MCL were not included in this cohort. 4) In IHC, P27 was more frequently expressed in the good prognostic group (14/17) than in the poor group $(22 / 60)[p=0.0022]$. Expression of CXCR4 showed no difference between the two groups (10/ 17 vs. 39/60) $(p=0.85)$. Cases with high Ki-67 labeling (more than $40 \%$ ) were more frequent in poor prognostic group $(19 / 60)$ than the other $(3 / 17)(p=0.40)$.

Conclusions: We investigated the clinicopathologic characteristics of MCL among Koreans and evaluated diagnostic utility of Sox 11, which showed higher sensitivity than cyclinD1. Pleomorhpic/blastoid cytomorphology and diffuse pattern were related to adverse prognosis, likely previous reports. The IHC prognostic panel [low p27, high CXCR4, and high Ki-67] can be also expected a poor prognosis. 


\section{LYS092}

Prognostic phenotypic and genotypic in situ biomarkers in diffuse large-B cell lymphomas: preliminary translational report of the prospective SAKK 38/07 trial

Stephan Dirnhofer ${ }^{1}$; Alexander Tzankov ${ }^{2}$; Nora Leu ${ }^{2}$; Simone Muenst ${ }^{2}$; Dirk Klingbiel ${ }^{2}$; Christoph Mamot $^{2}$

(1) Pathology, University Hospital Basel, Switzerland (2) Institute of Pathology, University Hospital Basel, Switzerland

Background: Diffuse large-B cell lymphoma (DLBCL) exhibits variable outcomes and risk assessment is based on the international prognostic index (IPI), which takes into account primarily patient-related parameters. The prognostic role of tumor-related parameters is a matter of controversy. Aim of the study: We aimed to prospectively analyze the prognostic value of phenotypic and genotypic profiles suggested in retrospective studies to play a role in DLBCL on a clinical trial collective of patients homogenously treated with six cycles of rituximab, cyclophosphamide, doxorubicin, vincristine, prednisone, followed by 2 cycles of Rituximab (R-CHOP). Materials and methods: Evaluation of the prognostic role of positron emission tomography (PET) was the main objective, and was performed before, after 2 cycles of therapy and at the end of treatment. Immunohistochemical (BCL2, BCL6, CD5, CD10, CD20, CD95, CD168, Cyclin E, FOXP1, GCET, Ki67, LMO2, MUM1p, pSTAT3) and in situ hybridization analyses [BCL2 break apart probe (BAP), C-MYC BAP and C$\mathrm{MYC} / \mathrm{IgH}$ double-fusion probe (DFP) and Epstein-Barr virus probe (EBER)] were performed and correlated with response to therapy defined by Cheson's criteria and defined by PET.

Results: 124 patients were analyzed. The median patients' age was 59 years; 68 were males, 56 females. Immunohistochemistry succeeded in all cases, while FISH was successful only in 65 instances, primarily due to poor tissue preservation of core needle biopsies and fixation biases. Taking into consideration the expression of CD10, GCET, FOXP1, LMO2 and MUM1 according to the so called Tally algorithm, 82 cases $(66 \%)$ were classified as non-germinal center (GC) DLBCL, while 42 cases (34\%) were GC DLBCL. 46/124 cases (37\%) expressed BCL2 in $>50 \%$ of tumor cells, 22 cases $(18 \%)$ expressed BCL6 in $>50 \%$ of tumor cells, 5 cases $(4 \%)$ were CD5 positive, 2 cases $(2 \%)$ were EBV infected, 7 cases $(6 \%)$ expressed RHAMM in $>12 \%$ of tumor cells, 21 cases $(17 \%)$ expressed Cyclin E in $>20 \%$ of tumor cells, 44 cases (35\%) expressed CD95 in $>12 \%$ of tumor cells and 31 cases $(25 \%)$ expressed pSTAT3 in $>12 \%$ of tumor cells; the mean proliferative activity, assessed by Ki-67, was $58 \%$. BCL2 gene breaks were observed in $7 / 65$ cases $(11 \%$ ), and those cases also expressed BCL2 in a mean of $95 \%$ of tumor cells, compared to a mean expression of BCL2 in $42 \%$ of tumor cells in non- rearranged instances; 6 of the rearranged cases were of the GC type. Two cases (all of the non-GC type) showed BCL2 amplifications. C-MYC breaks were observed in 6/65 cases (9\%); 4 were of the GC phenotype. Of the C-MYC rearranged cases only 2 displayed C-MYC/IgH fusions, detectable by both DFP and $\mathrm{BAP}$ and corresponding to $t(8 ; 14)$, while $\mathrm{C}-\mathrm{MYC}$ rearrangements were detectable only by BAP in the other 4 cases and were thus assumed to have occurred with alternative nonIgH C-MYC rearrangement partners. Cases with rearranged $\mathrm{C}-\mathrm{MYC}$ showed as high $\mathrm{Ki}-67$ labeling as non-rearranged ones. Cases with both BCL2 and C-MYC rearrangements ("double-hits") were not observed. A complete response (CR) defined by Cheson's criteria was observed in 86 out of 118 patients, while by PET 90 out of 118 patients showed CR, for 6 there were no data. Factors that were linked to failure to achieve PET-CR were CD5 positivity $(60 \%$, i.e. $3 / 5$, compared to $22 \%$, i.e. $25 / 113$ in the CD5 negative group, $p=$ $0.051)$, EBER positivity (100 \%, i.e. $1 / 1$, compared to $23 \%$, i.e. $27 / 117$, in the EBER negative group, $p=0.072$ ) and presence of either BCL2 or C-MYC gene rearrangements (39\%, i.e. $5 / 13$, compared to $18 \%$, i.e. $6 / 34$, in non-rearranged cases, $p=0.132$ ), but neither IPI nor phenotypic subtype according to the Tally algorithm. Analogous results were observable for failures to achieve CR defined by Cheson's criteria.

Conclusion: Poorly processed/preserved, especially small core needle biopsies, which are still sufficient for diagnostic and phenotypic studies in DLBCL, may hamper FISH-based studies. Phenotypic and genotypic studies with carefully selected biomarkers like CD5, EBER and BCL2- as well C-MYC rearrangement status might identify though small, yet prognostically relevant DLBCL subgroups with adverse outcomes under R-CHOP. A final evaluation will be done after the primary clinical end-point ( 3 years after completion of R-CHOP) will have been reached.

\section{LYS093}

The alteration of lipid metabolism in Burkitt Lymphoma identifies a novel diagnostic marker: adipophilin

Maria Raffaella Ambrosio ${ }^{1}$; Pier Paolo Piccaluga ${ }^{2}$; Maurilio Ponzoni $^{3}$; Bruno Jim Rocca ${ }^{4}$; Valeria Malagnino ${ }^{5}$; Monica Onorati $^{4}$; Giulia de Falco ${ }^{1}$; Valeria Calbi ${ }^{6}$; Martin Ogwang ${ }^{6}$; Kikkeri N. Naresh ${ }^{7}$; Stefano A. Pileri ${ }^{8}$; Claudio Doglioni ${ }^{9}$; Lorenzo Leoncini ${ }^{1}$; Stefano Lazzi ${ }^{1}$

(1) Department of Human Pathology and Oncology, University of Siena, Siena, Italy (2) Haemolymphopathology - S. Orsola-Malpighi Hospital - University of Bologna (3) Institute of Surgical Pathology, Ospedale San Raffaele (4) University of Siena (5) Univerisy of Siena (6) Saint Mary Hospital (7) Imperial College (8) Univerisy of Bologna (9) University Scientific Institute San Raffaele, Italy 
Background: Recent evidence suggests that lipid pathway is altered in many human tumours and in Burkitt lymphoma (BL) this is reflected by the presence of lipid droplets which are visible in the cytoplasm of neoplastic cells in cytological preparations but not on histological preparations. The formation, maintenance, modification and involution of lipid droplets is crucially regulated by a family of lipid dropletassociated proteins, to which belong five members, usually referred to as PLIN proteins.

Aim of the study: In this study, by analyzing the lipid metabolism, we attempted to find a novel marker that could detect lipid vacuoles in histological samples.

Materials and methods: We investigated the expression of genes involved in lipid metabolism in $47 \mathrm{BL}$ and 33 not-BL at both RNA and protein levels. We analyzed Gene expression profile (GEP) data of $13 \mathrm{BL}$ and $20 \mathrm{DLBCL}$ and performed immunohistochemical evaluation with adipophilin antibody on $47 \mathrm{BL}$ and 33 not BL classified according to the scoring system recently designed by Naresh et al. for aggressive B-cell lymphomas.

Results: GEP indicated a significant over-expression of the adipophilin gene and marked up-regulation of other genes (SCD5, FASN and USF1) involved in lipid metabolism in BL. These findings were confirmed by immunohistochemistry: 45 out of $47 \mathrm{BL}$ cases showed strong adipophilin expression characterized by single or multiple droplets in the cytoplasm and clustering of these to the outer nuclear membrane while only 3 cases of the 33 of the not-BL category showed weak adipophilin expression $(p<0.05)$.

Conclusions: Our results suggest that accumulation of lipid vacuoles is due to an altered lipid metabolism in BL. The vacuoles may be specifically recognized by a monoclonal antibody against adipophilin, which may therefore be a useful marker for the diagnosis of $\mathrm{BL}$ because of its peculiar expression pattern. Moreover this peptide might represent an interesting candidate for interventional strategies.

\section{LYS094}

\section{The BCL2 E17 and SP66 antibodies discriminate two immunophenotypically and genetically distinct subgroups of conventionally BCL2 "negative" grade I/II follicular lymphomas}

Patrick Adam ${ }^{1}$; Rosalie Baumann ${ }^{2}$; Irina Bonzheim ${ }^{3}$; Janine Schmidt $^{1}$; Falko Fend ${ }^{3}$; Leticia Quintanilla-Martinez ${ }^{3}$

(1) Institute of Pathology, University Hospital Tübingen, Germany (2) Institut of Pathology, University Hospital Tübingen, Eberhard-Karls-Universität Tübingen (3) Institute of Pathology and Neuropathology, University Hospital Tuebingen, Eberhard-Karls-University
Background: Follicular lymphoma (FL) is characterized by the chromosomal translocation $t(14 ; 18)(q 32 ; \mathrm{q} 21)$ resulting in constitutional overexpression of the antiapoptotic protein BCL2. However, in $10-15 \%$ of FL grade I/II immunohistochemical (IHC) staining for BCL2 remains negative. Recently, an alternative BCL2 antibody (clone E17) has been reported to be positive in part of these "negative" FL cases.

Aim of the study: The aim of this study was to analyze in a large series of FL grade I/II the incidence of BCL2 pseudonegativity and the underlying molecular mechanisms.

Materials and methods: $240 \mathrm{FL}$ grade I/II were included in the study. Immunohistochemical analysis was performed with three different BCL2 antibodies. (clones 100D5, E17, SP66), and with the germinal center markers LMO2 and HGAL. The presence of a $t(14 ; 18)$ was determined by FISH using a BCL2 break-apart probe. Additionally, exon 1 of the BCL2 gene, where the epitope of the standard BCL2 antibody (100D5) resides, was sequenced.

Results: Of the 240 cases of FL grade I/II identified, 22 cases $(9.2 \%)$ were negative with the standard BCL2 antibody. Of these, 12 cases ( $55 \%$ ) carried a BCL2 break indicative of a $t$ $(14 ; 18)$, and all but one case showed BCL2 positivity with both alternative BCL2 antibodies (E17 and SP66). All 12 cases showed a characteristic FL phenotype with strong expression of CD10, BCL6 and absence of CD23 in the tumor cells. Mutation analysis of BCL2 exon 1 revealed missense mutations with a hot spot around codon 144 in 9 analyzable cases. Ten ( $45 \%)$ of the 22 cases showed no abnormality of the BCL2 gene locus by FISH, which correlated with E17/ SP66 negativity. Six of these cases showed an aberrant FL phenotype, three were CD10 negative and three expressed CD23. All cases were $\mathrm{LMO} 2$ and HGAL positive. Two E17/SP66 negative cases carried a BCL6/IGH translocation. Mutational analysis of BCL2 showed a wild type sequence in all E17/SP66 negative cases.

Conclusions: Staining with the alternative BCL2 antibodies E17/SP66 separates two immunohistochemically and genetically distinct subgroups of BCL2 negative FL grade I/II. E17/SP66 positivity strongly correlates with the presence of the $t(14 ; 18)$ and missense mutations in exon 1 of the BCL2 gene. The molecular pathogenesis of the truly BCL2 protein, and $t(14 ; 18)$ negative FL grade I/II ( $4 \%$ of the cases) remains to be determined.

\section{LYS095}

Prevalence of EBV+ diffuse large cell lymphoma of the elderly in Argentina. A preliminary report

Marina Ines Narbaitz ${ }^{1}$; M. Cohen ${ }^{2}$; E. DeMatteo ${ }^{3}$; F. Agost Carreño $^{1}$; Maria Victoria Preciado $^{3}$; Paola Chabay $^{3}$; Carlos A. Martín ${ }^{4}$ 
(1) Pathology Department, National Academy of Medicine, Buenos Aires (2) Molecular Biology Lab \& Pathology Department, Dr. R. Gutierrez Children's Hospital, Buenos Aires. (3) Molecular Biology Lab \& Pathology Department, Dr. R. Gutierrez Children's Hospital, Buenos Aires. (4) Hematopathology Consultation

Background: The presence of Epstein-Barr virus (EBV) in a group of adult cases of Diffuse Large B-cell Lymphoma (DLBCL) without a known cause of immunosuppression has been described and included as a provisional entity in the 2008 WHO lymphoma classification.

Aim of the study: To present our preliminary data on EBV+ prevalence in a series of adult cases of DLBCL from Argentina. Materials and methods: The slides from 32 adult ( $>50$ years) DLBCL cases were retrieved from the files of National Academy of Medicine Pathology Department. Thirteen males and 19 females, with a mean age of 68,6 years (range 51-83 years), all immunocompetent, were selected. H\&E slides were reviewed and classified according to 2008 WHO classification, and further characterized as polymorphous or monomorphous. EBV status was assessed by means of in situ hybridization (ISH) technique for EBV RNAs (EBERs). Only cases showing a majority of cells positive for EBERs were considered EBV+DLBCL.

Results: Twenty-six tumors were nodal and 6 extranodal. Polymorphic subtype (23 cases) predominated over monomorphic subtype ( 9 cases). Necrosis was present in 13 cases. Eight of 32 patients (25\%) showed EBERs+ tumor cells (4 males and 4 females, mean age 69,5 years). Six tumors were nodal and 2 extranodal, 6 were of the polymorphic and 2 monomorphic subtype. In only two patients $(6,5 \%)$, a 76 year old male and a 68-year old women both with nodal disease, the majority of tumor cells were EBERs+, being both tumors of polymorphic morphology.

Conclusions: The results show a higher prevalence of $\mathrm{EBV}+\mathrm{DLBCL}$ in our patient population compared to previous reports from USA and Europe, and very similar to those from Mexico and Asian countries. Again, higher prevalence of EBV infection of the population and ethnicity could explain the geographic differences. Further analysis of more cases is needed to complete these preliminary results.

\section{LYS096}

\section{Histiocytic Sarcoma: histological and} immunohistochemical analysis and study of apoptosis

Cristiano C. Oliveria ${ }^{1}$; Rafael B. Paschoalini ${ }^{1}$; Fabíola E. Rosa $^{1}$; Rodrigo M. dos Santos ${ }^{1}$; Maria A. C. Domingues ${ }^{1}$

(1) São Paulo State University (UNESP)
Background: Histiocytic Sarcoma (HS) is a rare malignant neoplasia with morphological and immunophenotypic characteristics similar to mature tissue histiocytes. Its morphological heterogeneity overlaps with other malignancies and is accompanied by numerous clinical and epidemiological variations, making the entity difficult to diagnose. We propose that apoptotic pathways may be compromised in HS pathogenesis, inducing neoplastic histiocyte cell death, which, even when well differentiated, presents diverse morphological features.

Aim of the study: To improve the definition of HS pathogenesis, associated with frequent morphological patterns and specific immunomarkers, in an attempt to assure appropriate pathological diagnosis and complement current morphological criteria and immunophenotypic markers.

Materials and methods: Three confirmed cases, received by our service between 1998 and 2009, were evaluated using an immunohistochemical panel configured with 19 markers.

Results: All three HS cases presented 12 relevant morphological criteria: medium to large cell size, vacuolated chromatin, irregular membrane, intranuclear inclusion, vacuolar eosinophilic cytoplasm, bizarre-shaped cells, cohesiveness, epithelioid characteristics, phagocytosis, stroma with inflammatory infiltrate, apoptosis and hemosiderin. Concerning the panel of 19 immunohistochemical markers, 5 of these showed similar results in all three cases: CD163; CD68; vimentin and lysozyme, with diffuse distribution; and focal S-100. CD45 and CD30 were positive only in the skin sample. Regarding markers for disease pathogenicity, two of these showed positive results in all three cases, Fas-ligand and caspase-3.

Conclusions: The literature affirms that ligand expression in some tumours could be evidence of a proinflammatory response. Microenvironment alterations due to inflammatory processes can promote tumour growth and its immune response evasion. Analysis of the results suggests the existence of external factors related to the microenvironment that lead to tumour induction, such as infection.

\section{LYS099}

Primary Extranodal Lymphoma of the Thyroid: Case Series

Maurício F. S. A. Ribeiro ${ }^{1}$; Jéssica E. Fulfaro ${ }^{1}$; Thais S. de Oliveira $^{1}$; Cristiano C. Oliveira ${ }^{1}$; Maria A. C. Domingues ${ }^{1}$

(1) São Paulo State University (UNESP), Brazil

Background: Lymphomas consist of malignant neoplasias of the lymphoid system with both nodal and extranodal manifestations. One possible primary site of extranodal lymphomas is the thyroid.

Aim of the study: Determine the subtypes of primary thyroid lymphomas diagnosed at the Clinics Hospital in 
Botucatu and characterize these regarding epidemiological features and clinical evolution.

Materials and methods: Anatomopathological reports from 1998 to 2011 were reviewed and cases with a morphological diagnosis of primary lymphoma of the thyroid were selected. Clinical and epidemiological data were investigated by reviewing patient medical charts.

Results: Four patients were selected: three women and one man, aged 22 to 74 years-old; the main complaint of all four patients was increased cervical volume ( 3 after 3 months and 1 after 4 months); three presented B symptoms; two presented Hashimoto Thyroiditis; and two autoimmune thyroid disease without hypothyroidism. Regarding location, one patient presented the disease bilaterally and the other three on the right lobe. Of these, one presented substernal goiter. Considering disease stage, two presented stage VB, one IE and one IIIB. Analysis of histological subtype revealed that three neoplasias were diffuse large B-cell lymphomas and one was a lymphoblastic T-cell lymphoma. Currently, two patients are being administered chemotherapy treatment and are in good general health and two are in full remission.

Conclusions: Primary thyroid lymphomas are rare, representing less than $1 \%$ of all lymphomas. They present B phenotype, manifest in advanced stages and respond well to chemotherapy, indicating that the microenvironment may determine the aggressiveness of this neoplasia.

\section{LYS100}

\section{Impact of the microenvironment on the prognosis} of primary bone diffuse large B cell lymphoma

Ágota Szepesi ${ }^{1}$; Hajnalka Rajnai ${ }^{1}$; Botond Tímár ${ }^{1}$; Fenna Heyning $^{2}$; Lienna Koens ${ }^{2}$; Judit Csomor ${ }^{2}$; András Matolcsy ${ }^{3}$

(1) SE I. Department of Pathology (2) Leiden University Medical Center, The Netherlands (3) Dept. Genetics, Cell-\& Immunobiology, Semmelweis University

Background: Primary non-Hodgkin lymphoma of the bone (PLB) is a rare neoplastic disorder, comprising $5 \%$ of extranodal lymphomas. Eighty percent of the PLB cases are diffuse large Bcell lymphomas (PBDLBCL). Overall, PLB carries a favourable prognosis. Although the microenvironment has a significant influence on the tumour cell growth, outcome of the disease and response to treatment, PBDLBCL has not been characterized with regards to the infiltrating reactive immune cells.

Aim of the study: To determine the prognostic significance of tumour cell microenvironment on the outcome of the disease and possible association with the germinal centre$\mathrm{B}(\mathrm{GC}-\mathrm{B})$ and activated $\mathrm{B}$ cell (ABC) phenotype.

Materials and methods: Forty-five cases of PBDLBCL diagnosed at the I. Department of Pathology Semmelweis
University and Department of Pathology Leiden University were studied. The GC-B versus ABC subtype was determined by Tally's immunostaining algorithm based on the expression of GCET1, CD10, MUM1, FOXP1 and LMO2 antigens. Different components of the microenvironment, such as $\mathrm{T}$ cell subsets and macrophages of the microenvironment were analyzed by immunohistochemistry using tissue microarrays (TMA).

Results: A group of 45 patients with PBDLBCL was studied. Twenty-nine patients had single bone infiltration, while multifocal bone involvement (scored as stage IV) was noted in 11 cases. The mean IPI score was 1.8 . The overall survival was $80 \%$ with a median follow up of 51 months. Most patients received combination therapy in the form of surgical resection, poly-chemotherapy and radiotherapy. Thirty-two patients (71\%) had a complete remission (CR), 9 patients died of the disease (20\%), 4 had progressive disease (PD), 5 had partial response (PR) and died during therapy. There was significant association between age and survival: 7 out of 9 patients $>60$ years of age died of disease. Stage and tumour cell phenotype did not show significant effect on survival. The number of tumour infiltrating Tlymphocytes represented by CD3 positive cells showed significant correlation with the outcome and with the phenotype of the tumour cells $(p<0,01)$. Significantly higher number of tumour infiltrating CD8+ cytotoxic $\mathrm{T}$ cells and lower proliferation rates were observed in cases with $\mathrm{CR}$ compared to the patients with PR or PD disease $(p<0,01)$. Conclusions: Our cohort of PBDLBCL patients had favorable outcome, age at disease onset, tumour infiltrating CD3 $+\mathrm{T}$ cells and CD8+ cytotoxic T-cells significantly correlated with patients' survival. The finding of a positive impact of tumour infiltrating T-cells in DLBCL is in accordance with earlier reports of nodal lymphomas, however our study is the first showing association between microenvironment and disease outcome for PBDLBCL.

\section{LYS101}

A systematic comparison of the cell-of-origin immunohistochemistry algorithms in a large single-centre cohort of patients with Diffuse Large B-cell Lymphoma

Rita Coutinho ${ }^{1}$; Andrew James Clear ${ }^{2}$; Andrew Owen ${ }^{2}$; Andrew Wilson ${ }^{2}$; Janet Matthews ${ }^{2}$; Abigail Lee ${ }^{2}$; John G. Gribben $^{2}$; Maria Calaminici ${ }^{2}$

(1) Barts Cancer Institute (2) Department of HaematoOncology, Barts Cancer Institute, Queen Mary University

Background: Diffuse Large B-cell Lymphoma (DLBCL) is a molecularly heterogeneous disease. Activated B-cell 
DLBCL patients have a more aggressive behaviour and are being offered targeted therapies. Molecular discrimination is being performed by gene expression arrays in clinical trials. In clinical practice, IHC is still the method of choice.

Aim of the study: We set out to compare the performance of all immunohistochemistry (IHC) classification algorithms and correlate results with survival in an independent dataset.

Materials and methods: Tissue Microarrays (TMA) were constructed from 218 diagnostic biopsies: 128 males, 90 females, with a median age of 55 (18-94) years; $23.2 \%$ high-int/high risk IPI; median follow up of 3 years. 72 of the patients were treated with rituximab (R). Staining was performed to classify the patients according to the following algorithms: Hans, Hans modified, Choi, Choi modified, Nyman, Muris, Tally and Natkuman. Percentage positivity for each antibody using the criteria established in the original papers was agreed among 3 observers (RC, AL, MC). Consensus matrices were built. Interrater reliability analysis (Kappa) was performed to determine consistency among algorithms. Survival analysis was performed using the Kaplan Meier method and log rank test.

Results: Although all patients could be classified by at least one algorithm, we were only able to classify 172 of the patients for all algorithms studied. Not all algorithms allocate the same patients to either GCB or non-GCB groups. More patients were allocated to the non-GCB group and the level of concordance was higher for the non-GCB group among all algorithms. $17 \%$ of the patients were allocated to the non-GCB groups by all algorithms except one - either the Choi, Natkuman, or Muris algorithms, which are the methods that attribute more patients into the GCB subset. The highest level of agreement was found between the Hans and the Muris algorithms (Kappa $=0,69 ; p<0,001)$. From all the methods applied the Hans and the Hans modified exhibited the highest consistency with the remaining algorithms. The Hans, Hans modified, Muris and Choi modified algorithms show that GCB patients have a significantly better overall survival (OS), progression free survival (PFS) and disease-specific survival (DSS).

Discussion: In the absence of concomitant gene expression data, we are unable to determine which of the algorithms is the best surrogate for molecular subclassification in DLBCL.

We believe our cohort is enriched for patients in the nonGCB group, and have excluded that this could be due to a higher number of elderly patients.

We have used a statistical method to evaluate the degree of reliability among algorithms and found that the levels of consistency are low. Only $15 \%$ of patients were homogeneously classified by them all. It is important to emphasize that we are the first to compare all the 8 strategies published, which obviously increases the probability of miscoring. We have shown that the level of agreement in allocating patients to the non-GCB group is higher among all methods. It has been demonstrated by other groups that the proportion of misclassified cases by IHC algorithms compared with gene expression profiling was higher when defining the GCB subtype.

We demonstrate that it is possible to discriminate 2 subroups of patients with distinct prognosis using IHC algorithms. In this cohort none of the algorithms was significantly correlated with other prognostic factors, such as IPI or rituximab (R) treatment. Consistently, patients in the GCB group have a better outcome. The Hans algorithm appears to be useful for prognostic discrimination in the $\mathrm{R}$ era. We found that GCB patients treated with chemoimmunotherapy have a significantly better DSS $(p<0,05)$. However, due to the low number of patients in this group we are underpowered to detect statistically significant prognostic differences for OS $(p=0,06)$ and PFS. This study will now be extended to a new cohort of $\mathrm{R}$ treated patients. These results are useful for clinicians that wish to inform their patients about disease prognosis.

\section{LYS102}

\section{Hodgkin-like immunoblast proliferation in lymph node of patients treated with fludarabine for low grade B-cell neoplasm}

Tatjana Terzic ${ }^{1}$; Olivera Markovic ${ }^{2}$; Predrag Miljić3 ; Maja Peruničić-Jovanović ${ }^{4}$; Vladimir Jurišić ${ }^{5}$

(1) Institute of Pathology School of Medicine University of Belgrade (2) KBC "Bezanijska kosa", Belgrade (3) Clinic for Hematology, Clinical Center of Serbia, Belgrade (4) Department for Histopathology, Clinical Center of Serbia, Belgrade (5) School of Medicine University of Kragujevac

Background: Biopsy of lymph node of patients with low grade B-cell lymphoma was performed in cases of suspicious disease progression. But, pathohistology of lymph node after fludarabine therapy may be very atypical, sometimes with Hodgkin-like morphology.

Aim of the study: We present three cases which highlight diagnostic difficulties in suspicious transformation of low grade B-cell lymphoma after fludarabine therapy.

Materials and methods: The diagnosis was obtained on review of hematoxylin and eosin stained paraffin-embedded slides and immunohistochemical data (LCA, PAX5, CD79?, CD20, CD30, CD15, MUM-1, IgM, p53, EMA, CD5, CD10, CD23, CD43, bcl-2, cyclin D1, CD38, CD138).

Results: We present three cases (one follicular lymphoma and two chronic lymphocytic leukemias) with very unusual morphology of lymph node after fludarabine therapy. In all three cases there was no evidence of transformation in 
lymph node, but morphology was very similar to Hodgkin lymphoma (mononuclear and multinuclear cells, with ReedSternberg like cells). But, immunohistochemical analysis excluded diagnosis of Hodgkin lymphoma. These cells were positive for LCA, PAX5, CD79?, CD20, CD30, MUM-1, IgM, p53, and negative for CD15, EMA, CD5, CD10, CD23, CD43, bcl-2, cyclin D1, CD38, CD138. These large atypical cells phenotypically represented B-immunoblasts. The surrounding lymphoid tissue lost specific immunophenotypic features of follicular lymphoma and chronic lymphocytic leukemia (except B-cell markers), which may occur after chemotherapy. This non-neoplastic immunoblast proliferation in chronic lymphocytic leukemia is necessary to be carefully considered because transformation of CLL to Hodgkin lymphoma after fludarabine therapy is possible, but rare $(0.5-2 \%)$.

Conclusions: The post treatment lymph node evaluation in low grade B-cell non-Hodgkin lymphoma can lead to a mistaken diagnosis of Hodgkin lymphoma, especially when the clinical data of preexisting diagnosis and therapy of NHL was absent.

\section{LYS103}

\section{Incidence of framework 2 and framework 3 Immunoglobulin Heavy Chain Genes}

\section{Zohreh Mohammad Taheri ${ }^{1}$; Leila Ziazi ${ }^{2}$; Atoosa Drodinia ${ }^{3}$;} Forozan Mohammadi ${ }^{4}$

(1) NRITLD (2) MSD (3) MD, Pathologist (4) MD, Professor of Pathology, NRITLD, Shahid Beheshti University of Medical Science

Background: Identification of gene rearrangements and clonality analysis are important techniques for the diagnosis of malignant lymphoproliferative diseases. These methods have various sensitivities based on the type of primer used and method of determination of polymerase chain reaction (PCR) products.

Aim of the study: This study aimed at determining the clonality of B cell non-Hodgkin lymphoma in Iranian patients using PCR method and 2 primers of FR2 and FR3 and comparison of two methods.

Materials and methods: Paraffin embedded blocks of 67 patients with B cell lymphoma and 19 cases with lymphoid hyperplasia of the lymph nodes who presented to NRITLD, Masih Daneshvari Hospital were retrospectively reviewed. After extracting the DNA genome using phenol and chloroform, clonal analysis was performed using semi-nested PCR and 2 primers of FR 2 and FR3. PCR products were determined using 2 techniques of heteroduplex analysis, polyacrylamide gel and silver staining and the conventional method of agarose gel and ethidium bromide staining. Appearance of 1 or 2 bands in the desired location were considered as a sign of clonality. Results: Monoclonal gene rearrangement was observed in 62 out of 67 patients $(92.5 \%)$ as one or two discrete bands appeared within 60-120 base pairs (bp) and 200-300 bp range. Of the mentioned patients, 53 cases $(79.1 \%)$ had FR2 and $51(76.1 \%)$ had FR3 rearrangement. Heteroduplex analysis along with silver nitrate staining detected 3 out of the remaining 5 cases of lymphoma to be monoclonal. These cases had been reported negative by the conventional technique. In total, 65 out of 67 patients $(97 \%)$ showed monoclonal gene rearrangement using both the above mentioned techniques. False negative cases included 2 cases of large cell lymphoma.

Conclusions: Our study showed that evaluation and detection of clonality using PCR, FR2 and FR3 primers along with heteroduplex analysis is a rapid sensitive technique for the diagnosis of malignant lymphomas

\section{LYS104}

\section{Intraocular metastases of testicular lymphoma}

Metka Volavsek ${ }^{1}$; Andreja Brozic ${ }^{2}$; Natasa Vidovic Valentincic $^{3}$; Katrina Novak Andrejcic ${ }^{3}$; Mojca Globocnik Petrovic $^{3}$; Veronika Kloboves-Prevodnik ${ }^{2}$

(1) Institute of Pathology, Faculty of Medicine, University of Ljubljana, Slovenia (2) Department of Cytopathology, Institute of Oncology (3) University Eye Hospital, University Medical Centre

Background: Primary testicular lymphomas (PTL), although rare, are the most common testicular tumors in men older than 60 years. The majority of PTL are diffuse large B-cell lymphomas (DLBCL). There is predilection for dissemination of PTL to extranodal and atypical sites i.e. central nervous system (CNS), Waldeyer's ring, lung and skin, warranting careful evaluation of especially CNS and contralateral testis. Ocular manifestation of PTL is extremely rare.

Aim of the study: To describe morphological and immunophenotypic features of metastatic ocular PTL and compare them to the features of primary testicular lymphoma.

Materials and methods: At the Department of cytopathology, Institute of oncology Ljubljana Slovenia, five cases of metastatic intraocular lymphomas were diagnosed from 2004 to 2011. Two of these were metastatic PTL. Morphological and immunophenotypic features of metastatic and primary PTL were reviewed.

Results: The first was a case of 67-year old man with DLBCL of a left testis, Stage I.A.E. He was treated with orchidectomy and 4 cycles of R-CHOP. Complete remission was achieved. 17 months after completion of treatment, he 
presented with intraocular mass without involvement of CNS. Diagnostic vitrectomy was performed. Since metastatic DLBCL was diagnosed cytologicaly, the patient was treated with 5 cycles of R-CHOP and 1 cycle of R-COEP, high dose Methotrexate, intrathecal chemotherapy and radiotherapy of both eyes. At the end of the treatment only partial remission was achieved. The second case was the case of 74-year old man with DLBCL of left testis, stage I.A. After orhidectomy he was treated with 6 cycles of R$\mathrm{CHOP}$ and intrathecal chemotherapy. One year later, similarly to the previous case, intraocular mass was detected in his right eye. Cytological examination of vitrectomy specimen showed metastatic DLBCL. Therefore, the patient was treated with RCHOP, high dose Methotrexate, intrathecal chemotherapy and radiotherapy to both eyes. In March 2012 complete remission was achieved. Cytological examination of vitreous specimen of both cases showed large lymphoma cells with pale basophilic cytoplasm and large oval or lobulated nuclei with nucleoli and finely granular chromatin. In addition to relatively rare lymphoma cells numerous degenerated naked nuclei, some lymphocytes and histiocytes were present. Flow cytometric immunophenotyping (FCI) of both cases showed few large cells which were monoclonal, CD19+, CD20+, FMC7+, CD10-, CD5- and CD23-. Monoclonal cells in the first case were kappa + and in the other lambda + . Comparison between lymphoma cells from vitrectomy specimens to those present in primary testicular tumors revealed noticeable lobulation of the nuclei only in cytology. Results of immunophenotypic analyses could not be directly compared, since different panels of antibodies were used in cytology and histology.

Conclusions: The results of our small series of metastatic ocular lymphoma showed PTL as the origin of intraocular metastases in about $40 \%$, suggesting eyes as another extra nodal site of PTL metastatic spread, needing special attention. Since in ocular specimen only few lymphoma cells mixed with reactive background can be present, morphological and flow cytometric analyses are very demanding and have to be carried out only in specialized cytological laboratory. FCI of primary testicular lymphomas should be performed in all cases to obtain the immunophenotypic features of lymphoma cells. These data could be of great help in interpretation of FCI results of poorly cellular ocular specimens.

\section{LYS105}

\section{T-cell clonality patterns in immunodeficiency related lymphoproliferative disorders}

Han van Krieken ${ }^{1}$; B. Tops ${ }^{1}$; Konnie Hebeda ${ }^{1}$; Patricia Groenen ${ }^{1}$

(1) Pathology Department, Radboud Univeristy Nijmegen Medical Centre, The Netherlands
Background: Immunodeficiency-related lymphoproliferative disorders (LPDs) are a diverse group of proliferations of Epstein-Barr infected B-lymphocytes categorized as arising in primary immunodeficiency, HIV related, posttransplantation (PTLDs), and other iatrogenic/medication related LPDs. Within these groups LPDs are heterogeneous and can range from relatively benign disease without the need for clinical interference (infectious mononucleosislike, IM), to high-grade malignant lesions with very aggressive clinical behavior that need immediate treatment. This is generally accompanied by a polyclonal rearrangement pattern of the B-cell antigen receptors in the benign part of the spectrum and a monoclonal pattern in the malignant cases. Aim of the study: In these LPDs it is not uncommon to detect clonal T-cell populations as well, but the incidence and the relevance of this finding is not known.

Materials and methods: Using the full set of primers for the T-cell receptors genes of the Biomed2 program we analysed in total 38 EBV driven B-cell LPDs.

Results: We found in 2/4 casus of IM a weak clonal T-cell population; in 3/7 medication related LPDs a clear monoclonal T-cell population and in 1 case multiple clones; in 2/4 primary immunodeficiency associated LPDs a clonal T-cell population and in 1 multiple clones; in PTLD 2/15 had a clonal T-cell population, but 11 had multiple clones. There was no relation between the result of the T-cell receptor clonality testing and outcome. None of the patients developed a T-cell lymphoma.

Conclusions: T-cell clonality testing in EBV-positive LPDs in the various forms of immunodeficiencies commonly shows a clonal result, even in IM, which does not indicate the presence of a T-cell lymphoma nor indicates a poor outcome. The presence of a clonal T-cell population in EBV-positive B-cell LPDs needs to be interpreted with the full knowledge of the histology, the immnophenotype and the clinical background.

\section{LYS106}

TheJUNB/CD30 axis contributes to cell cycle deregulation in ALK+ anaplastic large cell lymphoma

Vassilis Atsaves ${ }^{1,2}$, Lazaros Lekakis ${ }^{1}$, Elias Drakos ${ }^{1}$, Vasiliki Leventaki ${ }^{1}$, Dan Jones ${ }^{1}$, Marianna Feretzaki ${ }^{1}$, Chrysoula Liakou', Anita Korapati ${ }^{3}$, Ryouichi Horie ${ }^{4}$, Panayiotis Panayiotidis ${ }^{5}$, Vassilis Gorgoulis ${ }^{6}$, Efstratios Patsouris $^{2}$, L. Jeffrey Medeiros ${ }^{1}$, Francois-Xavier Claret ${ }^{3}$, and George Z. Rassidakis ${ }^{1,2}$

Departments of Hematopathology ${ }^{1}$, and Systems Biology ${ }^{3}$, The University of Texas M.D. Anderson Cancer Center, Houston, Texas; First Department of Pathology ${ }^{2}$, First Department of Propedeutic Medicine, ${ }^{5}$ and Laboratory of Histology 
and Embryology, ${ }^{6}$ National and Kapodistrian University of Athens, Greece; ${ }^{4}$ Fourth Department of Internal Medicine, Kitasato University School of Medicine, Kanagawa, Japan

Background: ALK + anaplastic large cell lymphoma (ALCL) is a CD30+ T-cell non-Hodgkin lymphoma that frequently carries the $t(2 ; 5)(\mathrm{p} 23 ; \mathrm{q} 35)$ leading to overexpression and activation of the NPM-ALK oncoprotein. JunB, a member of the c-Jun family of AP-1 transcription factors, is overexpressed in ALCL and interacts with the CD30 gene promoter thus inducing CD30 expression. Particularly in ALK+ALCL, JunB is thought to be upregulated at the transcriptional level through ERK/Ets-1 and at the translational level through mTOR pathway. However, the mechanisms of JunB function in ALK+ALCL are not yet known.

Material and methods: Four ALK+ALCL cell lines (Karpas299, SR-786, SU-DHL1 and DEL) and control cells (Jurkat) were used. Tissue specimens from 6 previously untreated patients with NPM-ALK+ALCL were analyzed for JunB gene amplification as assessed by quantitative real-time PCR. JunB and CD30 gene silencing experiments were based on transient transfections with specific siRNA's. Cell viability and proliferation were assessed using trypan blue and MTS assays, respectively. Cell cycle and apoptosis studies were based on BrdU incorporation and Annexin $\mathrm{V}$ staining, respectively, analyzed by flow cytometry. Cell clonogenic capacity was assessed by colony formation assay. Western blotting was used for protein detection. Gene promoter activity was evaluated by luciferace assay. AP-1 activity was assessed by E.M.S.A.

Results: Here we demonstrate for the first time that $\operatorname{JunB}$ gene is frequently amplified in ALK+ALCL. We also show that both JunB and CD30 contribute to AP-1 activity and cell proliferation in NPM-ALK+ALCL through regulation of cell cycle. Silencing of JunB gene results in reduced colony formation associated with G1/ $\mathrm{S}$ and $\mathrm{G} 2 / \mathrm{M}$ cell cycle arrest, downregulation of cyclins A, D2 and D3 and upregulation of CDK inhibitors p14 and p21 in NPM-ALK+cells. Similar cell cycle changes were observed following knocking-down CD30 gene or inhibition of its function using anti-CD30 antibodies. Interestingly, these effects on cell cycle were mediated by the same CDK inhibitors, p14 and p21. In addition to cell cycle inhibition, treatment with anti-CD30 antibodies led to significant apoptotic cell death through regulation of anti-apoptotic or pre-apoptotic molecules. Silencing of JunB gene also sensitized ALCL cells to standard chemotherapeutic agents. Moreover, preliminary data show that JunB seems to interact with other oncogenic signaling pathways through novel crosstalk mechanisms.
Conclusions: Taken together, our findings uncover the oncogenic role of JunB gene in ALK+ALCL. JunB seems to function as an oncogene through deregulation of cell cycle in a CD30-dependent or CD30-independent manner. Moreover, our data further support the use of anti-CD30 targeted therapies in ALCL.

\section{LYS107}

Clonality testing for the T-cell receptor genes in the duodenum: clonality does not implicate malignancy

Han van Krieken ${ }^{1}$; Konnie Hebeda ${ }^{1}$; Patricia Groenen ${ }^{1}$ (1) Pathology Department, Radboud Univeristy Nijmegen Medical Centre, The Netherlands

Background: Refractory celiac disease is defined as confirmed celiac disease that does not respond to gluten free diet. About $50 \%$ of these patients will develop enteropathy associated T-cell lymphoma (EATL) and will die of this complication. It is presently difficult to predict which patient with refractory celiac disease may develop EATL and may therefore benefit from early treatment.

Materials and methods: We used the complete set of Biomed2 primers for the T-cell receptor genes to test the hypothesis that clonal refractory celiac disease indicates the development of EATL

Results: From the 18 cases of refractory celiac disease 10 were clonal, but there was no correlation with outcome. Furthermore, our results in our control groups were of note. As expected, 4/4EATL were clonal, but also 4/10 uncomplicated celiac disease cases and 3/9 normal duodenal biopsies.

Conclusions: We conclude that clonality testing is not a reliable method to indicate aggressiveness in refractory celiac disease, and that the results need to be interpreted in the context of all clinical and histological findings.

\section{LYS108}

CD4+ $\mathbf{T}$ cell expansion in lymphomatoid granulomatosis: a result of interleukin-10 release by Epstein Barr virus-associated $B$ cell proliferation

Cervera Pascale ${ }^{1}$; Fabiani Bettina ${ }^{1}$; Rossi Benjamin ${ }^{1}$; Guihot Armelle ${ }^{1}$; Gorochov Guy ${ }^{1}$; Buffet Marc ${ }^{1}$; Lassoued Kaiss ${ }^{1}$; Coppo Paul ${ }^{1}$

(1) Department of pathology, Hopital Saint-Antoine Paris, France

Background: Lymphomatoid granulomatosis (LG) is characterized by an EBV-driven B cell proliferation with a 
polyclonal CD4+ T-cell expansion, which mechanism still remains unclear.

Aim of the study: To better characterize the CD4+ T-cell expansion in LG.

Materials and methods: A 56-year-old female developed features of LG 1 year after a course of rituximab for a cold agglutinin disease. The number of EBV-specific CD4+ Tcells was measured by Elispot using EBV latency and lytic proteins. Functional analysis of blood lymphocytes was performed after stimulation with usual mitogens and microbial antigens. Serum interleukin (IL)-10 was quantified by ELISA.

Results: The frequency of CD4+ T-cells directed against EBV latency proteins was $<1 \%$. Moreover, the number of CD4+ T-cells expressing interferon-gamma obtained after EBV antigens exposure was undetectable, ruling out an EBV-driven CD4+ T-cell expansion. A mechanism involving the EBV HERV-K18 superantigen was excluded since Vbeta5 and Vbeta13 $+\mathrm{T}$ cells were not expanded. T-cell proliferation rates were normal following stimulation with mitogens. By contrast, there was no T-cells proliferation with the recall infectious antigens, consistent with an anergic state of T cells. Finally, IL-10 level was dramatically increased on diagnosis. By contrast, IL-10 level decreased 130-fold 3 months after treatment. The search of IL-10-secreting EBV-positive B-cells in bone marrow is ongoing.

Conclusions: We hypothesize that in our patient, EBVdriven B cells expanded along with the immune B-cell reconstitution that occurred 1 year after the course of rituximab, and resulted in a release of high levels of IL10. Consequently, IL-10 led to the expansion of anergic CD4+ T-cells with lung involvement. The remarkable response of our patient to rituximab and steroids is consistent with this view. These results could help better understanding the pathophysiology of diseases with a CD4+ T-cell expansion such as LG and the drug reaction with eosinophilia and systemic symptoms (DRESS).

\section{LYS109}

\section{Granulomatous Slack Skin: Report of a case}

Papoudou-Bai Alexandra; Bai Maria

University of Ioannina, Greece

Background: Granulomatous slack skin (GSS) is an extremely rare type of cutaneous $\mathrm{T}$ cell lymphoma characterized by slow development of folds of lax skin in the major skin folds and histologically by a granulomatous infiltrate with $\mathrm{CD} 4+\mathrm{T}$ cells, macrophages and multinucleated giant cells (WHO, 2008).
Case report: A 41-year-old man presented with a 1-year history of growing asymptomatic large soft tissue masses accompanied by pendulous lax skin in the upper thighs bilaterally and the left inguinal region. MRI confirmed the soft tissue masses and showed bilateral enlargement of inguinal lymph nodes. Biopsies were taken from both lesions and the diagnosis of GSS was made. The dermis and subcutis were massively and diffusely infiltrated by small to medium-sized lymphocytes, with slight pleomorphism. Unusually large multinucleated giant cells were evenly distributed within the infiltration, and contained numerous intracytoplasmatic lymphocytes. There were no epithelioid cell granulomas or epidermotropism of lymphocytes. Destruction of elastic fibers in the zone of massive infiltration was demonstrated by elastic Van Gieson staining. Immunohistochemistry revealed that tumor cells were $\mathrm{CD} 2+, \mathrm{CD} 3+, \mathrm{CD} 5+, \mathrm{CD} 7+, \mathrm{CD} 43+, \mathrm{CD} 45 \mathrm{RO}+$, CD8- and CD30-. The proliferation index MIB1 was $\sim 10 \%$. Histopathological examination of one removed inguinal lymph node showed reactive lymphadenopathy. The patient responded poorly to 6 months treatment with bexarotene.

Conclusions: Only limited data on the clinicopathological, and prognostic features of GSS are available. Histopathological overlap between GSS and granulomatous mycosis fungoides (GMF) has been reported in the literature, but it is debated whether GSS is a variant of MF, as proposed by WHO classification, or whether it is a distinct disease.

\section{LYS111}

Chronic Lymphocytic Leukemia/Small Lymphocytic Lymphoma metastatic to mature cystic teratoma of the ovary: Case report

Mohamed M. Desouki ${ }^{1}$; Hanaa A. El Sayed ${ }^{2}$

\section{(1) Vanderbilt University (2) MUSC (USA)}

Background: Teratomas, one of germ cell tumors of the ovary, are composed of mature or immature tissues deriving from one or more germ cell layers. More than $80 \%$ of malignant transformations of teratomas reported are squamous cell carcinomas especially in postmenopausal patients. The incidence of malignant transformation is 1.2 to 14.2 cases per 100,000 people per year. The incidence of metastatic or primary lymphoma is very small in ovarian teratomas.

Aim of the study: We report a very rare case of metastatic chronic lymphocytic leukemia/small lymphocytic lymphoma to mature cystic teratoma of the ovary.

Results: Out of 983 cases of ovarian teratomas retrieved in our institution from 2002 to 2011, only one case found to have metastatic chronic lymphocytic leukemia/small lymphocytic 
lymphoma in the ovarian teratoma. The patient is a 57-year-old Caucasian woman with history of CLL for few years presented with thrombocytopenia, peripheral blood lymphocytes which was treated as autoimmune phenomena with prednisone and left adnexal mass identified on CT scan examination. The later is a 4-5 cm mass in the area of the left adnexa suspected to be mature cystic teratoma with no adenopathy or evidence of ascites and normal sized spleen. Physical examination is remarkable for obesity with a BMI of 38. Pelvic examination is within normal limits. The patient underwent total abdominal hysterectomy and bilateral salpingo-oophorectomy with no immediate postoperative complications. On gross examination, the left adnexum consist of a previously collapsed ovarian cystic structure measuring $5.5 \times 4.5 \times 2.8 \mathrm{~cm}$. The outer surface is pinktan and smoothly lobulated with an attached portion of fimbriated Fallopian tube. The inner lining is predominantly smooth with patchy tan-brown areas and a $1.0 \times 0.8 \times 0.5 \mathrm{~cm}$. raised, pink-tan nodule. Cyst contents are cloudy, pink-tan with possible sebaceous material. The attached Fallopian tube measures $5.0 \mathrm{~cm}$. in length and averages $0.6 \mathrm{~cm}$. in diameter with a smooth, pink-tan serosa. Microscopic examination revealed mature benign cystic teratoma elements infiltrated with neoplastic lymphoid cells. The neoplastic cells are CD5, CD43, PAX-5 and CD23 positive with variable CD20 expression and tested negative for CD10 and Cyclin-D1 by IHC. As an incidental finding, the fallopian tube harbored focal high grade intraepithelial neoplasia with strong focal P53 staining (P53 signature). Conclusions: To the best of our knowledge, this is the first case reported of CLL/SLL metastasizing to mature cystic teratoma of the ovary. Accurate histological evaluation and clinical correlation are keystones of successful diagnosis.

\section{LYS112}

\section{Annexin.A1 expression in a case of Splenic Diffuse Red Pulp small B-cell Lymphoma}

Larissa Sena Teixeira Mendes; Estella Matutes; Ayoma Attygalle; Andrew Wotherspoon

Royal Marsden Hospital, Fulham Road, London SW3 6JJ, United Kingdom

Annexin-A1, also known as lipocortin, is encoded by the ANXA1 gene. It was the first characterized member of the annexin family of proteins, being involved in a broad range of cellular processes, including inflammatory signaling, apoptosis and differentiation. The ANXA1 gene was found to be one of the most upregulated genes in the hairy cell leukemia and in the context of neoplastic B cell lymphoproliferations, positive staining for Annexin-A1 is considered to be a specific marker of this condition.

Splenic lymphomas primarily involving the red pulp include hairy cell leukaemia (HCL), hairy cell leukaemia variant
(HCLv) and splenic diffuse red pulp small B-cell lymphoma (SDRPSBCL). Histologically, there is some morphological overlap between these entities, thus ANXA1 staining is used as a specific discriminator between HCL and the other lymphomas involving splenic red pulp.

Here, we describe a case of a SDRPSBCL with unexpected ANXA1 expression in a 75-year-old female patient. Spleen sections showed a diffuse infiltration of the red pulp by a population of small to intermediate-sized cells, with round/ oval nuclei, clumped chromatin, and inconspicuous nucleoli; often surrounding blood lakes. The neoplastic cells showed a CD20+/DBA.44+/TRAP+/ANXA1+ immunoprofile. Further, bone marrow trephine analysis displayed a similar proliferation to that found in the spleen with a strikingly dominant intrasinusoidal pattern which is considered more characteristic of SDRPSBCL and HCLv, rather than HCL. In addition, no BRAF V600 mutations were detected by CE-SSCA.

We believe this is the first description of a case that, on histological grounds, corresponds to the new provisional entity SDRPSBCL (WHO, 2008) expressing Annexin-A1. This finding suggests that Annexin-A1 staining may not be entirely specific of HCL, and should be interpreted in the context of cell morphology, bone marrow histology and BRAF status.

\section{LYS113}

\section{Primary adrenal Diffuse Large Cell Lymphoma}

Vesna Cemerikic-Martinovic ${ }^{1}$; Olivera Markovic ${ }^{2}$; Tamara Martinovic ${ }^{2}$; Vesna Bozic ${ }^{2}$; Iva Paunovic ${ }^{2}$; Dragomir Marisavljevic $^{2}$

\section{(1) Beo-lab (2) KBC “Bezanijska kosa”, Belgrade}

Background: Primary adrenal lymphoma is extremely uncommon with only 120 cases have been reported worldwide. In contrast, secondary involvement of the adrenal glands by a non-Hodgkin lymphoma has been reported in as many as $25 \%$ of the patients with lymphoma. Most previously reported patients with primary adrenal lymphoma had poor prognosis, with propensity to recur in the CNS and other extranodal localization.

Aim of the study: We report the case of a 73-year-old men with the primary non-Hodgkin lymphoma of left adrenal gland with favorable clinical course.

Materials and methods: CT tomography of abdomen demonstrated $9 \times 7 \mathrm{~cm}$ mass of the left adrenal gland without splenomegaly or abdominal lymphadenopathy. Adrenal function was normal. Laparatomy with left adrenalectomy and splenectomy was done.

Results: Histological examination revealed diagnosis of a diffuse large B-cell lymphoma, non-germinal center type 
with following immunopfenotype: CK-,EMA-, Inhibin-, Synaptophysin-, LCA+, PAX5+, CD79+, CD20+, CD23-, CD10-, CD3-, CD5-, CD43-, CD30-, CD38-, CD138-, MUM-1, BCL2+, BCL6+ and ALK-1-. Ki-67 staining showed high proliferation index $(60 \%)$. MYC (8q24) rearrangement was identified by FISH. BCL2 and BCL6 were normal. The patient was treated with 6 cycles of R-CHOP chemotherapy with reduced dose of adriablastine because ejection fraction was deceased. In addition, CNS hemoprophylaxis (methotrexate) was given. PET/CT scans after therapy showed no residual disease. To date, the patient has been followed regularly for the past 8 months, with no evidence of tumor recurrence.

Conclusions: Primary adrenal lymphoma should be kept in mind in the differential diagnosis of adrenal masses.

\section{LYS114}

\section{A91V SNP in PRF1 gene is frequently found in NK/T-cell lymphomas}

Rebeca Manso-Alonso ${ }^{1}$, Socorro María Rodríguez-Pinilla ${ }^{2,3}$, Luis Lombardia $^{4}$, Gorka Ruiz de Garibay ${ }^{2,5}$, Maria del Mar López ${ }^{2,6}$, Lydia Sánchez ${ }^{7}$, Margarita Sánchez-Beato ${ }^{2,8}$, Miguel Ángel Piris ${ }^{2,9}$.

(1) Tumor Bank Unit, Fundación Jiménez Díaz, Madrid, Spain (2) Molecular Pathology Programme, Lymphoma Group. CNIO, Madrid. Spain (3) Pathology Department, Fundación Jiménez Díaz, Madrid, Spain (4) Clinical Research Program, Molecular Diagnostics Clinical Research Unit, CNIO, Madrid. Spain (5) Clinical Immunology Department, Hospital Clínico de San Carlos, Madrid, Spain. (6) Biotechnology Programme, Monoclonal Antibodies Unit, CNIO, Madrid, Spain (7) Biotechnology Program, Immunohistochemistry Unit, CNIO, Madrid, Spain (8) Onco-hematology Unit, Medical Oncology Department. Fundación Investigación Biomédica, Hospital Universitario Puerta de Hierro-Majadahonda, Madrid, Spain (9) Pathology Department, Hospital Universitario Marqués de Valdecilla, Universidad de Cantabria, IFIMAV, Santander, Spain.

Background: The 2008-WHO classification recognized the extranodal-NKTC-lymphomas (NKTCLs), aggressive-NKcell-leukemia, chronic-active-EBV-disease (CAEBV), EBVassociated hemophagocytic-lymphohistiocytosis (HFL), severe-mosquito-bite-allergy and hydroa-vacciniforme as neoplasms affecting T/NK-cells. PRF1 mutations are commonly present in familial-haemophagocytic-lymphohistiocytosis (FHL), and in a CAEBV case. FHL and EBV-associatedhemophagocytic-lymphohistiocytosis (EBV-HLH) have overlapping clinical manifestations, and CAEBV is often associated with EBV-HLH. Furthermore, most EBV-associated-LPD- patients evolve into proper NKTCL-lymphomas. The aim is to establish whether PRF1 mutations are related somehow to NKTCLs.

Material and methods: A series of 24 consecutive NKTCL cases were analyzed for a panel of 10 different antibodies and EBV-in-situ-hybridization. Exons 2 and 3 of the PRF1 gene were sequenced. Clinical and follow-up was also recorded.

Results: There were 11 males and 13 females whose median age at diagnosis was 56.6 years, ranging from 31 to 89 years. Lesions were located in the nasal-area (12), oropharynx (2), gastrointestinal tract (2), skin/subcutis (6), pleura (1) and testes (1). 19 patients received CHOP-like chemotherapy. Only five patients achieved complete-response, and 17 patients died $(80.95 \%)$ with a median survival of seven months (ranging from 1 to 45 months). EBER and CD3 were positive while nuclear-B-catenin and C-KIT were negative in all cases. Other markers were present in variable proportions: perforin: $95.8 \%$, CD56: $75 \%$, CD8: $27.3 \%$, CD8/CD4double positive: $9.09 \%, \mathrm{CD} 8 / \mathrm{CD} 4$-double negative: $63.63 \%$ and P53:20\%. The not previously described c. $289 \mathrm{G}>$ A (p.Ala97Thr) somatic mutation was reported in one case $(2.4 \%)$. Moreover, three of the tumors $(12,5 \%)$ had the g.272C $>$ T (p.Ala91Val) change, a SNP (rs35947132) implicated in loss of perforin protein function and related to a late onset FHL, as well as to lymphoblastic-leukemia and anaplastic-large-cell-lymphoma. All cases with these PRF1 SNPs were of nasal origin but no other correlation with clinical or inmunohistochemical data was found.

Conclusions: We report for the first time the correlation between nasal-NKTCLs and PRF1 gene alterations. Nevertheless, its implication in NKTCLs etiopathogeny needs further investigation.

\section{LYS115}

\section{Mutations in Treg and Th17 regulatory pathways in Mycosis Fungoides and Sezary Syndrome identified by massive parallel sequencing}

Margarita Sanchez-Beato ${ }^{1}$; Gonzalo Gomez ${ }^{2}$; Veronica Monsalbez ${ }^{1}$; Nerea Martinez ${ }^{3}$; Ignacio Varela ${ }^{4}$; Jose P. Vaque $^{3}$; Socorro M. Rodríguez-Pinilla ${ }^{5}$; Esperanza Martin ${ }^{6}$; Jose L. Rodriguez-Peralto ${ }^{7}$; Osvaldo Graña ${ }^{2}$; Orlando Dominguez $^{8}$; Miguel A. Piris ${ }^{9}$

(1) Instituto i+12. Hospital 12 de Octubre. Medical School. Universidad Complutense (2) Spanish National Cancer Research Center (CNIO), Structural Biology and Biocomputing Programme (3) Hospital U. Marqués de Valdecilla, IFIMAV, Cancer Genomics Laboratory (4) Instituto Biomedicina y Biotecnología de Cantabria (IBBTEC) (5) Fundacion Jimenez Diaz, Madrid (6) Spanish National Cancer 
Research Center (CNIO), Molecular Pathology Programme (7) Instituto i+12. Hospital 12 de Octubre. Medical School. Universidad Complutense. Spain (8) Spanish National Cancer Research Centre (CNIO), Biotechnology Programme (9) Hospital Marques de Valdecilla, Santander, Spain

Background: Cutaneous T-cell lymphoma (CTCL) is a heterogeneous group of diseases characterized by clonal expansion of malignant T-cells in the skin. Mycosis Fungoides (MF) and Sézary Syndrome (SS) are the most frequent CTCL. Molecular pathogenesis of CTCL is basically unknown. Some data suggest that signalling from T-cell receptor (TCR) is a driving force. However, molecular mechanisms responsible for this activation have not been clarified. Aim of the study: Based on the hypothesis that TCR activation may depend on somatic mutations, we investigated this in a selection of genes belonging to TCR, or related pathways, such as NFkB, JAK/STAT, by deep sequencing. Materials and methods: A Target Enrichment method using SureSelect system (Agilent) has been used to enrich in exons and regulatory regions of 524 genes belonging to these pathways.

DNA from 2 tumoral-MF, 5 erythrodermic-MF and 6 SS patients, both normal and tumoral, were processed and sequenced with Genome Analyzer GA2 (Illumina) (PE42bp). Sequencing data were first checked by FastQC for quality control checks on raw sequence data and then aligned to the human reference genome (GRCh37) using BWA and BFAST alignments. Somatic variants were identified using GATK. Thus, SNPs available at dbSNP 135 (hg19) and 1000 Genomes Project were filtered out from VCF output files. The GATK-QUAL field was employed for ranking selected somatic variants. Putative variants were manually reviewed and validated by capillary sequencing. Biological impact predictions for detected variants were obtained from Ensembl Variant Effect Predictor.

Results: Several mutations were found in essential genes belonging to pathways implicated in the Treg and Th17 regulatory pathways, NFkB and JAK/STAT, among others. Some genes were mutated in two or more samples.

Conclusions: Thus, our preliminary results show that TCR activation in MF and SS might be dependent of the acquisition of somatic mutations in the coding region of genes known to play an essential role in T-cell differentiation processes.

\section{LYS116}

\section{Diffuse Large B-cell lymphoma mimicking primary ovarian carcinoma - report of two cases}

Agata Pakulniewicz ${ }^{1}$; Piotr Czapiewski ${ }^{2}$; Anna Justyna Szkop-Dominiak ${ }^{3}$; Juliusz Kobierski ${ }^{3}$; Dariusz Wydra ${ }^{3}$; Wojciech Biernat ${ }^{1}$
(1) Department of Pathology, Medical University of Gdansk, Poland (2) Department of Pathology, Medical University of Gdansk (3) Department of Gynecology and Gynecological Oncology

Background: Primary ovarian carcinoma is the most common malignancy disseminating within the peritoneal cavity in female patients followed by metastatic carcinoma developing in the gastrointestinal tract. Rarely other rare neoplasms may show a similar presentation. We report two cases of diffuse large B-cell lymphoma (DLBCL) with such a clinical appearance.

Materials and methods: The database was searched for primary lymphomas involving ovary with diffuse involvement of the peritoneal cavity.

Results: Two female patients, 40-year-old and 52-year-old, were found. Both of them had ascites, involvement of the female genital tract and peritoneal dissemination. CA-125 level was slightly elevated in both patients. The histopathological analysis revealed DLBCL in both cases. The prior clinical history was negative for hematopoietic malignancy. However, the former patient was HIV-positive on longlasting antiviral therapy, while the latter did not show any signs of immunosupression.

Conclusions: DLBCL can manifest as an ovarian tumor with dissemination into the peritoneal cavity, and closely mimic primary ovarian carcinoma. Such a clinical appearance should be be taken into account in immunodeficient patients.

\section{LYS117}

\section{A combined case of Chronic Lymphocytic Leukemia and 8p11 myeloproliferative syndrome}

Sinatkas Vaios ${ }^{1}$; Spanoudakis Michael $^{1}$; Papadakis Michael $^{2}$; Xylouri Irene ${ }^{2}$; Papadaki A. Helen ${ }^{2}$; Stathopoulos N. Efstathios ${ }^{2}$; Drakos Elias ${ }^{2}$

(1) PEPAGNH (2) University of Crete School of Medicine, Greece

Background: 8p11 myeloproliferative syndrome (EMS) is a rare hematologic malignancy characterized by chromosomal translocations involving FGFR1 located at 8p11-12 and myeloid and lymphoblastic leukemia/lymphoma associated with eosinophilia. EMS cases combined with other lymphoid malignancies have not been reported to date.

Aim of the study: We present a case of EMS combined with chronic lymphocytic leukemia/small lymphocytic lymphoma (CLL/SLL) in a 59-year-old man showing combined lymph node and bone marrow histology.

Materials and methods: H\&E histology and immunohistochemistry of $4 \mu \mathrm{m}$ formalin-fixed, paraffin embedded sections, as well as classical cytogenetic study were used. 
Results: The patient with a 7-years history of CLL/SLL, hypermutated immunoglobulin gene type, presented with thrombocytopenia, neutrophilia and lymphadenopathy. Bone marrow histology showed a $20 \%$ nodular infiltrate by CLL cells accompanied by $40 \%$ of myeloid blasts. The immunophenotype of the blasts was $\mathrm{CD} 34+, \mathrm{MPO}+, \mathrm{TdT}+, \mathrm{CD} 3-$, and CD20-. Lymph node histology revealed a composite histologic pattern including nodular areas, corresponding to $30 \%$ of the total, some with characteristics of proliferation centers, composed of CD20+, CD5+ and CD23+ CLL cells, surrounded diffusely by lymphoblasts. The immunophenotype of the lymphoblasts was TdT+, CD3+, CD4+, CD20-, MPO-, and CD117-. Areas with intermixed histologic pattern were also observed. Bone marrow aspirate cytogenetic analysis identified a $t(8 ; 13)(\mathrm{p} 12 ; \mathrm{q} 12)$. The patient died after severe gastrointestinal bleeding before receiving cytotoxic chemotherapy. Conclusion: This is the first reported case of EMS associated with CLL/SLL showing composite lymph node and bone marrow histology. Coexistence of these malignancies is probably incidental, although that remains to be verified by future epidemiologic studies.

\section{LYS118}

\section{"Signet-ring-cell" lymphoma with massive chylous ascites simulating effusion lymphoma}

Masaru Hosone ${ }^{1}$; Aimin Liu ${ }^{1}$; Hironori Katayama ${ }^{1}$; Masataka Tanno ${ }^{1}$; Zenya Naito ${ }^{1}$

\section{(1) Nippon Medical School}

Background: Signet-ring-cell (SRC) lymphoma is a rare morphological variant of mature lymphomas including follicular lymphoma, marginal zone lymphoma, diffuse large B-cell lymphoma and peripheral T-cell lymphoma. We report a case of SRC follicular lymphoma with massive chylous ascites simulating effusion lymphoma.

Material and methods: The case corresponded to a 58-yearold immunocompetent Japanese male, HIV/HHV-8 negative

Results: The patient presented with abdominal fullness due to massive acites $(3500 \mathrm{ml})$ and mesenteric lymphadenopathy. Primary cytology of ascites revealed numerous SRCs with cytoplasmic clear vacuoles. Peritoneal dissemination of SRC adenocarcinoma was strongly suspected. Flow cytometry (FCM) revealed; however, a small B-cell lymphoma in ascites simulating effusion lymphoma. A mesenteric lymph node biopsy and FCM were performed. The lymph node revealed a vaguely follicular growth pattern of mature-looking small lymphoid cells with numerous, scattered SRCs. These PASnegative SRCs were positive for CD10,CD20, and IgG/lambda-restricted. Linear $\mathrm{IgG} / \mathrm{lambda}$ deposition along the perimeter of the vacuoles was demonstrated. SRC variant of follicular
lymphoma/Grade 1-2 was diagnosed. Electronmicroscopic (EM) analysis showed that some vacuoles were directly connected with rough ER(RER)s. This finding suggests that these clear vacuoles were derived from expanded RERs.

Conclusions: SRC lymphoma is divided into two subtypes: Russell body type and clear vacuole type. The former usually includes PAS $(+) / \operatorname{IgM}$ and the latter PAS $(-) /$ IgG. Our EM analysis suggested that the clear vacuoles were made of expanded multi-fused RERs. Interestingly, in the process of vacuolar formation, accumulated IgG seems to be washed out and left only at the periphery of the vacuoles. On the contrary, IgM is well preserved through the process. The key difference between the two is still uncertain. In conclusion, this particular case of SRC lymphoma showed an unusual clinical presentation and EM analysis was useful to speculate the mechanism of IgGrelated vacuolar formation.

\section{LYS119}

\section{T-cell mediated immune gastritis after rituximab treatment for gastric MALT lymphoma}

Evangelia Stalika ${ }^{1}$; George Kanellis ${ }^{2}$; Apostolia Papalexandri ${ }^{3}$; Michalis Iskas ${ }^{3}$; Efimia Vrakidou; ${ }^{4}$ Maria Demonakou ${ }^{5}$; Achilles Anagnostopoulos ${ }^{3}$; Kostas Stamatopoulos ${ }^{3}$; Theodora Papadaki $^{6}$

(1) Hematopathology Department "Evangelismos" Hospital Athens, Greece (2) Hematopathology Department, Evangelismos Hospital (3) Hematology Department and HCT Unit, G. Papanicolaou Hospital (4) Hematology Department, Hygeia Hospital (5) Pathology Department, Sismanoglion General Hospital (6) Department of Haematopathology, Evangelismos Hospital, Greece

Background: Rituximab is a chimeric monoclonal anti-CD20 antibody widely used for the treatment of B-cell malignancies. It achieves significant B-cell depletion and is well tolerated with adverse events mostly occurring during infusion. Severe late side effects are rare; however, the full impact of Rituximab on the hematopoietic and immune systems remains unknown. Studies from our group have linked a syndrome of late-onset neutropenia (LON) developing in a proportion of lymphoma patients after Rituximab with the presence of expanded cytotoxic $\mathrm{T}$ cells with large granular morphology (T-LGL) and immunophenotype $(\mathrm{CD} 3+\mathrm{CD} 8+\mathrm{CD} 57+)$. On these grounds, we have proposed that this manifestation of marrow failure may be interpreted in the context of T-LGL-mediated autoimmune myelopathy/myelodyplasia, at least in a proportion of such Rituximab-associated LON cases.

Aim of the study: To expand the "repertoire" of immune manifestations associated with Rituximab by reporting a case 
of Rituximab-treated gastric MALT lymphoma who developed late-onset CD8+ T-cell mediated erosive gastritis.

Materials and methods: A 51-year-old male with gastric B cell lymphoma of the MALT type, negative for $\mathrm{t}(11 ; 18)$ (API2/MALT1), was treated with Rituximab (4/weekly doses of $375 \mathrm{mg} / \mathrm{m} 2$ ). Three months after treatment, a gastric biopsy was negative for residual lymphoma. A follow-up biopsy, 4 months later, revealed diffuse erosive gastritis. Multiple gastric biopsies were evaluated. The biopsy was examined morphologically (H\&E) and immunohistochemically with a broad spectrum of antibodies: CD20, CD79a, CD5, CyclinD1, CD23, BCL2, BCL6, CD10, CD3, CD4, CD8 and MIB-1. Bcell and T-cell clonality was assessed by PCR and heteroduplex analysis of immunoglobulin heavy chain $(I G H)$ and $\mathrm{T}$ cell receptor beta chain $(T C R \beta)$ gene rearrangements. PCR products were sequenced and analyzed using the International imMunoGenetics information system (http://imgt.cines.fr/) and more particularly the IMGT/V-QUEST tool.

Results: The gastric biopsy showed a very limited Blymphocytic infiltration that could not document relapse. PCR detected a faint monoclonal IGH rearrangement product of identical size to that obtained from the diagnostic sample. Of particular interest was the finding of T-lymphocytic gastritis, composed almost exclusively of CD8+ T cells, associated with apoptosis, ulceration, reactive non-specific inflammation and slight cytologic atypia, which, in only a single tissue sample, could be interpreted as epithelial dysplasia. PCR analysis revealed polyclonal TRBV-TRBD-TRBJ gene rearrangements. The polyclonal PCR product was subcloned and 42 random colonies were sequenced. Among 32 productive rearrangements, 19 TRBV different genes were utilized. Alignment of CDR3 sequences revealed four different clusters of identical rearrangements, each including from 2 to 4 cases and collectively accounting for 12/32 subcloned sequences.

Conclusions: Our findings further expand the spectrum of immune-mediated manifestations associated with Rituximab treatment for lymphoma. In addition, they emphasize the need to utilize extensive immunohistochemistry panels for $\mathrm{B}$ and $\mathrm{T}$ cells when handling biopsies of lymphoma patients post Rituximab in order to discriminate between residual or relapsing disease and small lymphocytic infiltrates of pure T-cell origin mimicking residual disease.

\section{LYS120}

\section{A comprehensive immunohistochemical approach to $A K T / m$ TOR pathway in mycosis fungoides}

Georgia Levidou ${ }^{1}$; Marina Siakantaris ${ }^{2}$; Theodora Papadaki ${ }^{3}$; Evangelia Papadavid ${ }^{4}$; Theodoros P. Vassilakopoulos ${ }^{5}$; Leonidas Marinos ${ }^{3}$; Vassiliki Nikolaou ${ }^{6}$; Aphrodite Economidi ${ }^{6}$; Christina Antoniou ${ }^{7}$; Efstratios Patsouris ${ }^{8}$; Penelope Korkolopoulou ${ }^{8}$
(1) Department of Pathology, Kapodistrian University of Athens, Medical School, Greece (2) 1st Department of Internal Medicine, University of Athens, Medical School, Laiko Hospital, Greece (3) Department of Haematopathology, Evangelismos Hospital, Greece (4) Department of Dermatology, Athens University Medical School, Attikon Hospital, Greece (5) Department of Hematology and Bone Marrow Transplantation, University of Athens, Medical School ,Lai (6) Department of Dermatology, Athens University Medical School, A. Sygros Hospital, Greece (7) . Department of Dermatology, Athens University Medical School, A. Sygros Hospital, Greece (8) Department of Pathology, University of Athens, Medical School, Greece

Background: Given the recent identification of the mTOR pathway, as a possible target for cancer therapy, the expression pattern of phosphorylated mTOR (p-mTOR) pathway components has attracted scientific interest in several neoplasms, including cutaneous lymphomas. However, published information regarding its significance in mycosis fungoides (MF) is limited.

Materials and methods: We analyzed immunohistochemically 54 skin biopsies (21 tumor and 33 plaque stage) from 50 patients with $\mathrm{MF}$, for whom clinical information was available. Paraffin embedded tissue was stained for p-mTOR, its upstream p-AKT, its downstream effectors p-p70S6K and p-4E-BP1, as well as for p-ERK1/2 (extracellular signal-related kinase 1/2) and the transcription factor Notch-1. The expression of these molecules was correlated with clinico-pathological features and survival.

Results: p-p70S6K expression was recorded in all cases, whereas the rate of positivity for the remaining molecules ranged from $52.8 \%$ to $67.3 \%$. p-mTOR was co-expressed with p-p70S6K in $67.3 \%$ of cases, but co-expression with other molecules was less common (27.4-38.5\%). Notch-1 displayed higher $\mathrm{H}$-scores in tumors than in plaques $(p=$ 0.0475 ), as previously demonstrated. The same applied to $\mathrm{p}-$ p70S6K, but this correlation was of borderline significance. Significant correlations were recorded between $\mathrm{p}-4 \mathrm{E}-\mathrm{BP} 1$ and $\mathrm{p}-\mathrm{p} 70 \mathrm{~S} 6 \mathrm{~K}(p=0.0019), \mathrm{p}-\mathrm{ERK}$ and $\mathrm{p} 4 \mathrm{E}-\mathrm{BP} 1 \quad(p=$ $0.0588)$, as well as between Notch-1 and p-p70S6K ( $p=$ $0.0001)$ and $\mathrm{p}-4 \mathrm{E}-\mathrm{BP} 1(\mathrm{p}=0.0003)$. The latter two correlations remained when plaques and tumors were analyzed separately. Notch1, p-4E-BP1 and p-p70S6K expression were associated with advanced stage. In univariate survival analysis increased p-p70S6K $(p=0.0174), \mathrm{p}-4 \mathrm{E}-\mathrm{BP} 1 \quad(p=$ $0.0500)$ and $\mathrm{p}$-AKT $(p=0.0198) \mathrm{H}$-scores adversely affected overall (OS) but not disease-free (DFS) survival in plaques, along with CD30 expression $(p=0.0004)$. In particular, blood clonality affected both DFS and OS, as well as time to disease progression $(p=0.0009, p=0.0031$ and $p=0.0216$ respectively). 
Conclusions: Activation of AKT-mTOR components appears to be common in MF plaques and tumors and may be implicated in disease progression.

\section{LYS121}

Persistent polyclonal B- cell lymphocytosis with splenomegaly : histopathological description of two cases

Azahara Martínez López ${ }^{1}$; Ana Burdaspal Moratilla ${ }^{2}$; César Miranda Vallina ${ }^{3}$; Juan Luis Alfonso Martín ${ }^{4}$; Ana Batlle López $^{5}$; Sonia González de Villambrosia ${ }^{5}$; Francisco Mazorra Macho ${ }^{6}$; Santiago Montes Moreno ${ }^{7}$; Miguel A. Piris ${ }^{5}$

(1) Hospital Marqués de Valdecilla. IFIMAV. (2) Department of Pathology, Hospital Universitario Ramón y Cajal (3) Department of Pathology, Complejo Hospitalario de Orense (4) Departament of Pathology, Complejo Hospitalario Insular Materno-Infantil (5) Department of Hematology, Hospital Universitario Marqués de Valdecilla (6) Department of Pathology, Hospital Universitario Marqués de Valdecilla. IFIMAV (7) Department of Pathology, Hospital Universitario Marqués de Valdecilla. IFIMAV

Background: Persistent polyclonal B-cell lymphocytosis (PPBL) is a rare entity characterized by a chronic, stable and polyclonal lymphocytosis. In peripheral blood binucleated lymphocytes is a feature, together with an IgM polyclonal band in serum immunoelectroforesis. Splenomegaly is an uncommon finding. The report of clonal cytogenetics abnormalities contrasts with the polyclonal nature of the process. Given the fact that the association of this process with splenomegaly is very infrequent, the histology of the spleen in these cases is poorly described.

Aim of the study: The aim of the study is to report the histopathological features of the spleen in two cases of PPBL with progressive splenomegaly.

Materials and methods: For this purpose, we select two cases with the diagnosis of PPBL and massive splenomegaly, providing a descriptive analysis of histopathological, immunohistochemical features and FISH analysis.

Results: Both patients were middle-age women, smokers and with a marked lymphocytosis $(>10.000 / \mathrm{mm} 3)$, including binucleated cells, in peripheral blood.

In both cases splenic architecture was barely preserved with mild expansion of white pulp without enlargement of the marginal zone area. Red pulp showed a massive intersticial and intrasinusoidal infiltration by B-cells $(\mathrm{CD} 20+, \mathrm{IgM}+$, IgD+, BCL2+, CD3-,CD5-, Ciclina D1-, BCL6-, CD10-, CD23-, CD43-, without light chain restriction ). We also noticed the presence of binucleated lymphocytes in splenic sinuses. BCL2 was not translocated in any of the cases, as demonstrated by FISH.

Conclusions: PPBL is a rare process with a benign clinical course. The histopathology of the spleen in cases with progressive splenomegaly is characterized by a mild polyclonal expansion of B-lymphocytes that shows an intersticial and intrasinusoidal infiltration of red pulp without involvement of white pulp. The unique architectural pattern and the absence of light chain restriction are the features that help to distinguish this condition from other types of splenic B-cell lymphoma.

\section{LYS122}

\section{Fibroblastic reticular cell tumor: a case report}

Maria Ap C. Domingues ${ }^{1}$; Rafael D. Gaiolla ${ }^{1}$; Cristiano C. Oliveira $^{1}$

\section{(1) São Paulo State University (UNESP)}

Background: Fibroblastic reticular cell tumour (FBRCT) is a very rare entity originating in stromal support cells located in parafollicular areas, the deep cortex of lymph nodes, in extrafollicular areas of the spleen and tonsils, and soft tissues.

Case report: The 56-year-old female patient presented with splenomegaly and enlarged perisplenic lymph nodes. CT scan showed a splenic mass measuring $7.0 \mathrm{~cm}$ in diameter; a perisplenic lymph node was biopsied. Fibroblastic reticular cell tumour was diagnosed. The patient was administered CHOP scheme and showed intrasplenic tumour regression. Remission was maintained at 8 months follow-up.

Results: Microscopy revealed a large, pleomorphic neoplasia with indistinct cell borders, eosinophilic cytoplasm in moderate quantities and delicate collagen fibres; whorled pattern spindle cells were observed. Immunophenotyping showed positive staining of cytokeratin network (dendritic pattern), 1A4, desmin and CD68; and negative for CD21, CD35 and S100 protein.

Conclusions: Fibroblastic reticular cell tumours present myofibroblastic-like characteristics and numerous clinical variations and frequently result in death of the patient.

\section{LYS123}

Clinicopathological characteristics and clinical course of CD8 expressing primary cutaneous peripheral T-cell lymphomas (CTCL) - a retrospective case study

Deniz Peker ${ }^{1}$; Yizhou Zhang ${ }^{2}$; Yong $\mathrm{Yu}^{2}$; Zhigang Zhao ${ }^{2}$; Yafei $\mathrm{Wang}^{2}$; Hongliang Yang ${ }^{2}$; Frank Glass ${ }^{1}$; Lynn Moscinski ${ }^{1}$; Lubomir Sokol ${ }^{1}$; Ling Zhang ${ }^{1}$ 
(1) H. Lee Moffitt Cancer Center (2) Department of Hematology, TianJin Medical University Cancer Hospital and Institute

Background: CD8+ primary cutaneous $\mathrm{T}$ cell lymphomas (PCTCL) are rare and basically include primary cutaneous CD8+ aggressive epidermotropic cytotoxic T-cell lymphoma (AECTL) and CD8+ variant mycosis fungoides (MF). In contrast to AECTL, which frequently exhibits strikingly aggressive clinical behavior, $\mathrm{CD} 8+\mathrm{MF}$ was reported to show a variable clinical course, from indolent to aggressive. The clinicopathological aspects of CD8+ PCTCLs are yet to be established.

Aim of the study: We retrospectively reviewed the clinicopathologic characteristics of CD8+ PCTCLs and correlated with clinical outcomes.

Materials and methods: The hematopathology files (PATHNET) at the H. Lee Moffitt Cancer\&Research Center and pathology archival files from Tianjian Cancer\&Research Institute, China, were retrieved. Cases with a primary diagnosis of CD8+ PCTCLs between January 2004 and June 2011 were included. All available histology slides, along with all of the ancillary study results were reviewed and correlated with the clinical data and survival.

Results: 10 cases of CD $8+\mathrm{MF}(n=5)$ and CD8+ non-MF $(n=$ 5, 2 cases with AECTL and 3 cases of CD8+ PCTCL) were included based on confirmed diagnosis. The morphology of the tumor cells varied from small to intermediate sized to large pleomorphic forms with variable growth patterns and epidermotropism in all cases. The overall survival time for CD8+ non-MF type (excluding 1 patient with lost follow up) varied from 5 to 90 months (averaging 20.5 months) while it was shorter in CD8+ MF with 5 to 23 months (average 12.6 months).

Conclusions: There is a major diagnostic challenge for CD8+ PCTCL with overlapping morphological and phenotypic features, particularly in MF with pleomorphism and disseminated disease. Larger series of CD8+ MF with gene profiling are warranted to establish genetic, molecular and phenotypic parameters not only to differentiate the subtypes, but also to distinguish from primary cutaneous CD8-positive AECTL.

\section{LYS124}

\section{EBV positive large B-cell lymphoma transformation} with extranodal marginal zone B-cell lymphomas

Nalan Akyurek ${ }^{1}$; Pnar Uyar Göçün ${ }^{1}$

(1) Gazi University, Medical Faculty, Department of Pathology

Background: Extranodal marginal zone B- cell lymphoma of mucosa-associated lymphoid tissue (MALT)- type is a mature B-cell neoplasm that typically follows an indolent clinical course. Despite its low aggressiveness, histological transformation to high-grade lymphoma has been described. Materials and methods: We describe the clinical and pathologic features of two cases of extranodal marginal zone Bcell lymphoma, which transformed into EBV-positive diffuse large B-cell lymphoma (DLBCL).

Results: The first patient was a 60-year-old man with extranodal marginal zone B- cell lymphoma of orbita. The patient did not receive any treatment and about approximately 5 months later cervical lymphadenopathy was developed. Lymph node biopsy showed large cell lymphoma. The second patient was a 67-year-old woman with thyroid lymphoma. Thyroidectomy specimen showed marginal zone B- cell lymphoma and large cell lymphoma characterized by infiltration of large, atypical, Hodgkin's-like cells.

The large cells showed CD20, CD30 and LMP-1 immunoreactivity in all cases. In situ hybridization for EBV-encoded RNA (EBER) was also positive.

Conclusions: The finding of EBV in the large cell lymphoma, but not the low-grade component suggests that EBV may have played a role in large cell transformation.

\section{LYS125}

Nonspecific cutaneous T-cell lymphoma rich in giant cells: a case report

Thais S. de Oliveira ${ }^{1}$; Cristiano C. Oliveira ${ }^{1}$; Maria Ap C. Domingues $^{1}$

\section{(1) São Paulo State University (UNESP)}

Background: Peripheral, nonspecific T-cell lymphomas are neoplasias with innumerous morphological subtypes corresponding to distinct clinical aspects, renowned for their difficulty to include them in specific entities. The report highlights a rare association of this entity with granulomatous reaction in multinucleated giant cells, which could obscure the main diagnosis.

Results: A 28 year-old Caucasian male presented infiltrated erythematous violaceous lesions, $20 \mathrm{~cm}$ in diameter on the right thigh and gluteus for 3 years. Examination revealed a right groin lymphadenopathy measuring $2 \mathrm{~cm}$ in diameter, negative for B symptoms. The initial clinical hypothesis was non-Hodgkin Lymphoma. Microscopy revealed an infiltrated lymphoid neoplasia occupying surface and deep dermis with destruction of adjoining tissues, constituted by small lymphocytes. Immunohistochemical studies were positive for $\mathrm{CD} 3$, CD4 in the lymphoid cells and CD68 and lysozyme in the giant cells. T-cell clonality proved positive by the genotyping method; BAAR and fungi tests were negative. The final diagnosis was peripheral (nonspecific) T-cell lymphoma rich in giant cells. The CHOMP scheme was indicated. 
Conclusions: Peripheral T-cell lymphoma is an entity that includes innumerous morphological aspects, though its association with granulomatous reaction rich in foreignbody type giant cells is rare. Presence of such cells can occur in other non-Hodgkin lymphomas, including B-cell lymphomas, angioimmunoblastic T-cell lymphomas and classic Hodgkin lymphoma. The presence of giant cells could lead to an inconsistent diagnosis of infectious disease, requiring the exclusion of specific agents using special stains and confirmation of lymphoproliferative disease through auxiliary studies.

\section{LYS126}

\section{Epstein-Barr Virus, CD20, CD3, and TIA-positive diffuse large cell lymphoma}

Miji Lee ${ }^{1}$; Hee Jeong Cha ${ }^{1}$; Geunyoung Jung ${ }^{1}$; Chan-Sik Park $^{1}$; Jooryung Huh ${ }^{1}$

\section{(1) Asan medical center, Seoul, Korea}

Background: T-cell associated antigens including CD5 and CD43 are regularly co-expressed in a subset of normal and malignant B-cells. Rare cases of B-cell lymphomas with $\mathrm{CD} 2, \mathrm{CD} 4, \mathrm{CD} 7, \mathrm{CD} 8$, or CD45RO have also been reported. However, co-expression of $\mathrm{CD} 3$ in $\mathrm{B}$-cell lineage lymphomas, or aberrant co-expression of CD20 in T-cell lineage lymphomas is extremely rare.

Results: A 72-year-old, HIV-negative, Korean female presented with epigastric discomfort and weight loss. Initial work-up revealed systemic lymphadenopathy and a $2 \mathrm{~cm}$ ulcerated mass in the stomach. Biopsies of the lymph node and the gastric mass showed diffuse sheets of large atypical lymphoid cells. The immunophenotype of the tumor cells was as followed: CD20 (+), CD3 (+), CD43 (+), CD79a (weak +), PAX-5 (weak +), MUM-1 (+), BCL2 (+), BCL6 $(-)$, BOB-1 (+), TIA-1 (+), OCT2 (+), CD15 (-), CD23 (-), CD10 (-), CD30 ( \pm ), TCRbetaF1 (-), CD2 (-), CD4 (-), CD5 (-), CD8 (-), Granzyme B (-), ALK-1 (-). In situ hybridization for EBER showed positive signals in the nuclei of the majority of tumor cells. HHV-8 (LANA) was negative. Molecular studies revealed dual rearrangements of immunoglobulin heavy (IgH) chain region and T-cell receptor (TCR) gamma genes. TCR beta gene rearrangement study failed to demonstrate monoclonal populations of T-cell. The case was diagnosed as Epstein-Barr virus-positive, CD3-positive, diffuse large B-cell lymphoma (CD3-DLBCL). Bone marrow examination showed infiltration of the tumor cells, assigning her to Ann Arbor stage 4B disease. She received RituximabCHOP regimen, with near total resolution.

Conclusions: This case illustrates the difficulty of lineage determination of non-Hodgkin lymphoma with only pan-B- and pan-T-cell markers. Unlike the previously reported cases, the present case showed molecular evidence of both $\mathrm{B}$-and T-cell lineages, which further complicates the interpretation. The association of CD3-DLBCL and Epstein-Barr virus warrants further study.

\section{LYS127}

TCR gamma expression in primary cutaneous T-cell lymphomas

Verónica Monsalvez ${ }^{1}$; Socorro M Rodríguez-Pinilla ${ }^{2,3}$; Pablo L. Ortiz-Romero ${ }^{4}$; Itziar Eraña Tomás ${ }^{5}$ Santiago Montes-Moreno ${ }^{2,6}$; Lydia Sánchez ${ }^{7}$; Luis Requena ${ }^{8}$; Jose L Rodríguez-Peralto ${ }^{9}$; Lorenzo Cerroni ${ }^{10}$; Miguel Á Piris ${ }^{2,6}$

(2) CNIO Lymphoma Group, Madrid, Spain (3) Fundación Jiménez Díaz, Pathology Department, Madrid, Spain $(1,4)$ Hospital Universitario 12 de Octubre, Dermatology Department, Madrid, Spain (5) Hospital deTorrejón de Ardoz, Pathology Department, Madrid, Spain (6) Hospital Universitario Marqués de Valdecilla, Pathology Department, Santander, Spain (7) Immunohistochemistry Unit, Biotecnology Programme, CNIO, Madrid, Spain (8) Fundación Jímenez Díaz, Dermatology Department, Madrid, Spain (9) Hospital Universitario 12 de Octubre, Pathology Department, Madrid, Spain (10) Research Unit Dermatopathology, Department of Dermatology, Medical University of Graz, Austria

Primary cutaneous $\gamma \delta$ - T-cell lymphomas are considered a subgroup of aggressive cytotoxic T-cell lymphomas. We have taken advantage of a new commercially available antibody recognizing the TCRGAMMA subunit of the TCR working on paraffin embedded tissue. We have analyzed a series of 146 primary cutaneous T-cell lymphomas received for consultation or second opinion to the CNIO pathology department. Cases were classified according to the WHO 2008 classification as mycosis fungoides (MF, $n=$ 96), primary cutaneous $\gamma \delta$ - T-cell lymphomas (PCGD-TCL, $n=5$ ); pagetoid reticulosis ( $\mathrm{PR}, n=6$ ); $\mathrm{CD} 30$-positive primary cutaneous anaplastic large cell lymphomas (C-ALCL, $n=$ 5); primary cutaneous CD8-positive epidermotropic cytotoxic T-cell lymphomas (PCCD8C-TCL, $n=3$ ), primary cutaneous cytotoxic TCL-NOS (PCCTCL, NOS, $n=4$ ), extranodal nasal-type NK/T-cell lymphomas affecting primary the skin or subcutaneous tissue $(\mathrm{NKTCL}=11)$ and lymphomatoid papulosis type D (LyP-D, $n=16$ ). In those positive cases for TCRGAMMA, a further panel of 13 antibodies was done, including TIA-1, granzyme B and perforin. Clinical and follow-up data were recorded in all cases. Twelve cases (8.2\%) resulted positive for TCRGAMMA, including 5 PCGD-TCL, two MFs, and 5 LyP-D. All 5 
PCGD-TCL and one MF patients died of the disease while the other MF patient and all patients with LyP-D were alive. All these cases expressed cytotoxic markers, were frequently CD3/CD8-positive and tended to lose CD5 and CD7. Eight out of 12 and five out of 11 cases were CD30 and CD56 positive, respectively. Interestingly, 5/12 TCRGAMMA positive cases also expressed TCRBF1. All cases analyzed were EBER negative.

Conclusion: TCRGAMMA expression seems to be rare, and to be confined to cytotoxic primary cutaneous T-cell lymphomas. Nevertheless, expression it's not exclusive of PCGD-TCL since TCRGAMMA protein can be found in other CTCLs. Moreover, expression does not seem to be associated with bad prognosis by itself, since it can be found in cases with good and bad outcome.

\section{LYS128}

Cytokine expression pattern of T-Cell neoplasms correlates with diagnosis and allows separation of peripheral T-Cell lymphomas in distinctive subgroups

Davide Soldini ${ }^{1}$; Santiago Mercadal $^{1}$; Teresa Cardesa ${ }^{1}$; Adriana Garcia ${ }^{1}$; Luis Colomo ${ }^{1}$; Carla Sole ${ }^{1}$; Blanca González ${ }^{1}$; Pedro Jares ${ }^{1}$; Elias Campo ${ }^{1}$; Armando Lopez-Guillermo ${ }^{1}$; Antonio Martinez ${ }^{1}$

(1) Hospital Clinic, IDIBAPS, University of Barcelona, Spain

Background: T-cell lymphomas (TCL) represent a heterogeneous group of neoplasms of innate and adaptive immune response. Cytokine expression profiles characterise a functionally complex spectrum of T-cell subsets, but exhaustive proteomic cytokine profiling has not been reported previously in TCL.

Design: A total of 72 TCL cases diagnosed between 1987 and 2012 at the Hospital Clinic were collected, and clinical and pathological data reviewed. Protein extracts of freshfrozen tissue biopsies were analysed with last-generation protein arrays (RayBio4 ${ }^{\circledR}$ Human Cytokine Antibody Array $G$ Series 2000) which allow simultaneous evaluation of 274 cytokines. Clustering analysis of normalised data was performed with dChip software.

Results: Following review, 72 TCL were classified as 14 AITL, 5 T-ALL, 13 PTCL-NOS, 14 ALCL (6 ALK+), 5 ATLL, 8 primary cutaneous (PC) PTCL, 7 primary cutaneous CD30+ LPD (4 LyP), 5 MF, and 1 NK/TCL. Unsupervised consensus clustering allowed identification of 4 different groups. In the largest one, which included most AITL and PTCL cases, several up-regulated cytokines were linked to angiogenesis, such as CXCL5, thrombopoietin, and angiopoietin-2. No clear signatures of B cells or $\mathrm{T}$ follicular helper cells were detected, possibly due to the high representation of the tumour microenvironment. The other groups included mostly T-ALL, ALK+ ALCL and PC CD30+ LPD, respectively. Consistent with their marked heterogeneity, PTCLNOS share cytokine profiles of different groups. The expression pattern identified some previously reported cytokines, whereas others are still poorly characterised.

Conclusion: TCL can be separated into different groups based on their cytokine expression patterns, which unveil potential functions of so far poorly studied cytokines. Cytokine expression profiling performed on protein array may become an important tool to improve the understanding of TCL heterogeneity and, in contrast to RNA profile studies, are of particular value as they overcome problems related to posttranscriptional and posttranslational modifications. 


\section{AUTHOR INDEX}

\begin{tabular}{|c|c|c|c|c|c|}
\hline Abion, F. & LYS005 & Bacci, F. & LYS003 & Bucher, C. & BMS013 \\
\hline \multirow[t]{2}{*}{ Adam, P. } & LYS012, LYS044, & Bachanova, V. & BMS010 & Bueso-Ramos, C. E. & BMS018 \\
\hline & LYS094 & Bai, M. & LYS109 & Buffet, M. & LYS108 \\
\hline Aerts, S. & LYS015 & Balatzenko, G. & BMS008 & Buggy, J. J. & LYS083 \\
\hline \multirow[t]{2}{*}{ Agostinelli, C. } & LYS001, LYS003, & Barrionuevo, C. & LYS019 & Buser, A. & BMS002 \\
\hline & LYS013 & Barth, T. F. E. & LYS023 & & \\
\hline Aguilar-Guadarrama, R. & LYS061 & Baseggio, L. & BMS005, LYS045 & Cairns, R. & LYS006 \\
\hline Aguilar-Leon, D. & LYS061 & Bastard, C. & LYS006, LYS041 & Calaminici, M. & LYS101 \\
\hline Aguilar, D. & LYS057, LYS059 & Baumann, R. & LYS094 & Calbi, V. & LYS093 \\
\hline \multirow[t]{2}{*}{ Aguilar, R. } & BMS022, LYS057, & Baxter, R. H. G. & LYS063 & Callet-Bauchu, E. & BMS005 \\
\hline & LYS059 & Beaty, M. & BMS011 & Campo, E. & LYS007, LYS128 \\
\hline Akkaya, B. & LYS062 & Becher, C. & LYS023 & Camps, J. & LYS033 \\
\hline Akyürek, N. & LYS043, LYS124 & Bedekovics, J. & BMS003 & Canioni, D. & LYS005, LYS073 \\
\hline Al-Darraji, A. F. & LYS078 & Begna, K. H. & BMS010 & Canoz, O. & LYS080 \\
\hline Al-Hadad, S. A. & LYS078 & Bellan, C. & LYS025, LYS079 & Capper, D. & BMS001 \\
\hline Al-Jadiri, M. F. & LYS078 & Bellas, C. & LYS076 & Capron, C. & LYS087 \\
\hline Alayed, K. & BMS018 & Bellosillo, B. & LYS071 & Cardesa, T. & LYS128 \\
\hline Alderete, G. & LYS020 & Benchekroun, S. & BMS006 & Carlotti, A. & LYS047 \\
\hline Alfonso Martín, J. L. & LYS121 & Bende, R. & LYS026 & Carreño, F. A. & LYS095 \\
\hline Aline-Fardin, A. & LYS087 & Benson, $\mathrm{M}$. & LYS054 & Casado, F. & LYS037 \\
\hline Allen, S. & BMS007 & Berger, F. & BMS005, LYS045 & Casavilca, S. & LYS019 \\
\hline Alsaadawi, A. R. & LYS078 & Berger, F. & LYS005 & Cazzola, M. & BMS004 \\
\hline Alvarez, J. L. & LYS071 & Bernard, O. & LYS006 & Cemerikic-Martinovic, V. & LYS113 \\
\hline Amador-Medina, F. & LYS059 & Bernd, H.-W. & LYS023 & Cerroni, L. & LYS127 \\
\hline Ambrosio, M. R. & LYS025, LYS093 & Bertuzzi, C. & LYS003, LYS013 & Cha, H. J. & LYS024, LYS126 \\
\hline Amé-Thomas, P. & LYS046 & Bi, Y. & LYS084 & Cha, H. J. & LYS091 \\
\hline Anagnostopoulos, A. & LYS119 & Biernat, W. & LYS116 & Chabay, P. & LYS095 \\
\hline Anastasiadu, E. & LYS078 & Bisig, B. & LYS011 & Chaby, G. & LYS047 \\
\hline Andersson, P. & LYS081 & Bittoun, E. & LYS033 & Chan, J. C. & LYS003 \\
\hline Andrejcic, K. N. & LYS104, LYS032 & Bogusz, A. M. & LYS063 & Chan, W. & LYS017 \\
\hline Andrulis, M. & BMS001 & Bonnet, C. & LYS011 & Chang, B. Y. & LYS083 \\
\hline Angeles, A. & LYS059 & Bonzheim, I. & LYS012, LYS020, & Chang, S.-T. & LYS065 \\
\hline Angelopoulou, M. K. & BMS021 & & LYS044, LYS050, LYS052, & Charlotte, F. & LYS005 \\
\hline Antoniou, $\mathrm{C}$. & LYS120 & & LYS094 & Chatelain, D. & LYS047 \\
\hline \multirow[t]{2}{*}{ Aralica, G. } & BMS017, LYS035, & Bosq, J. & LYS073 & Chauvin, J.-M. & LYS016 \\
\hline & LYS051 & Bossard, C. & LYS005, LYS016, & Chen, D. & BMS010 \\
\hline Arber, C. & BMS002 & & LYS018 & Cheng, A.-L. & LYS029 \\
\hline Arcaini, L. & LYS072 & Botargues, J. M. & LYS055 & Chenn, A. & BMS014 \\
\hline Arikan, S. & LYS062 & Bouchindhomme, B & LYS005, LYS018 & Chilosi, M. & LYS069 \\
\hline Arra, M. R. & LYS072 & Bouvet, M. & BMS024 & Chiu, A. & LYS042 \\
\hline Artchounin, C. & LYS046 & Boveri, E. & BMS004, LYS072 & Choi, S. J. & LYS024 \\
\hline Atsaves, V. & LYS106 & Bozic, V. & LYS 113 & Choi, Y.-D. & LYS024, LYS091 \\
\hline Attygalle, A. & LYS054, LYS112 & Briere, J. & LYS005, LYS038, LYS073 & Christensson, B. & LYS081 \\
\hline Atwater, S. K. & LYS058 & Brody, J. & BMS007 & Chu, C.-Y. & LYS029 \\
\hline Audisio, E. & BMS016 & Brousset, P. & LYS014 & Chuang, S.-S. & LYS065 \\
\hline Aulitzky, W. E. & LYS082 & Brozič, A. & LYS032, LYS104 & Claret, F.-X. & LYS106 \\
\hline Ayhan, A. S. & LYS043 & Brugieres, L. & LYS010 & Clear, A. J. & LYS101 \\
\hline
\end{tabular}


Climent, F.

Cogliatti, S.

Cohen, M.

Coiffier, B.

Colak, S.

Collie, A. M. B.

Colomo, L.

Comoz, F.

Condom, E.

Cools, J.

Copie-Bergman, C.

Coppo, P.

Corver, W. E.

Cotta, C. V.

Couronné, L.

Coutinho, R.

Croci, G. A.

Csomor, J.

Cunningham, D.

Currie, $\mathrm{T}$.

Czapiewski, P.

d'Amore, E. S. G.

d'Agay, M.-F.

D'Ardia, S.

Dallera, E.

Dartigues, $\mathrm{P}$.

De Anda-González, J.

de Castro, D. G.

de Falco, G.

de Garibay, G. R.

De Leo, A.

de Leval, L.

de Oliveira, T. S.

De Paepe, P.

de Rooij, M. F. M.

de Villambrosia, S. G.

De Wolf-Peeters, C.

Dechelotte, P.

Deffis-Court, M.

del Carmen Toledo, M.

del Mar López, M.

Delarue, R.

Delfau, M.-H.

Della Porta, M. G.

Delsol, G.

DeMatteo, E.

Demirkesen, C.

Demonakou, M.

Dengler, M. A.

Desfrançois, J.
LYS055

LYS023

LYS095

LYS073

LYS044

LYS031

LYS128

LYS047

LYS055

LYS015

LYS017, LYS038,

LYS046, LYS073

LYS087, LYS108

LYS077

LYS031

LYS006

LYS101

BMS004

LYS100

LYS054

LYS063

LYS056, LYS116

LYS010

LYS073

BMS016

LYS072

LYS005, LYS073

LYS061

LYS054

LYS025, LYS079,

LYS093

LYS114

LYS003, LYS013

LYS005, LYS006,

LYS011, LYS018

LYS086, LYS099,

LYS125

LYS015

LYS083

LYS121

LYS015, LYS033

LYS005

LYS059

LYS037

LYS114

LYS005

LYS018

BMS004

LYS005, LYS011

LYS095

LYS049

LYS119

LYS082

LYS016
Desouki, M. M.

Dhende, S.

Di Bello, C.

di Celle, P. F.

Di Lisio, L.

Di Napoli, A.

Diebold, J.

Dierickx, D.

Dikov, T.

Dimov, N. D.

Dirnhofer, S.

Dittman, D.

Dogan, A.

Doglioni, C.

Dojcinov, S.

Domingues, M. Ap. C.

Dominguez, O.

Dorfman, D. D.

dos Santos, R. M.

Drakos, E.

Drodinia, A.

Du, M.-Q.

Durán-McKinster, C.

Duranti, E.

Economidi, A.

Eker, C.

El Sayed, H. A.

Eldering, E.

Emile, J.-F.

Eom, D. W.

Epari, S.

Esen, T.

Espinet, B.

Estévez, M.

Estrach, T.

Eto, $\mathrm{H}$.

Fabiani, B.

LYS005, LYS006, LYS038, LYS073, LYS087, LYS108

Fataccioli, V.

LYS005, LYS014,

LYS041

Feller, A. C.

Felman, P.

LYS023

Fend, F.

BMS005, LYS045

LYS012, LYS020, LYS044,

LYS050, LYS052, LYS094

Feretzaki, M.

LYS106

Fernández-Rodríguez, C.

LYS071

Ferreiro, J. F.

LYS015

Ferrer, A.

LYS071

Ffrench, M. BMS005, BMS015, LYS045

Fiandrino, G.
LYS072
Fisch, P.

LYS056

BMS016

LYS048

LYS058

LYS044

LYS086, LYS099

Fulfaro, J. E.

Fuligni, F.

LYS003, LYS013, LYS079

Gaidano, G.

LYS072

Gaiolla, R. D.

Gallop-Evans, E.

LYS086, LYS122

LYS067

BMS004

LYS128

LYS076

Garcia, A.

Garcia, D.

Garcia, J. F.

Garcia, M.

LYS019, LYS085

LYS071

Gascoyne, R. D.

LYS002

Gaulard, P.

LYS005, LYS006,

LYS011, LYS014, LYS017,

LYS018, LYS038, LYS041, LYS046

LYS047, LYS073

Gazzo, S.

BMS005

Gazzola, A.

Geay, M.-O.

Geest, C. R.

LYS003, LYS013

BMS005

LYS083

LYS017

LYS038

LYS070

BMS004

LYS013

LYS038

LYS073

LYS058

LYS123

LYS045

LYS024

Glehen, A. T.

Go, $\mathrm{H}$.

LYS043, LYS124

BMS009

LYS115

LYS128

BMS014

LYS106

LYS108

LYS072

LYS115

Graña, O.

Gratwwohl, A.

Gratzinger, D.

Gribben, J. G.

Groenen, P.

Grube, $\mathrm{P}$.

Guenova, M.

Guettier, C.

Guihot, A.

Guikema, J.

Gujral, S.
LYS009

LYS101

LYS105, LYS107

LYS020

BMS008, LYS040

LYS073

LYS108

LYS026

LYS075 
Hageman, L.

Haioun, C.

Halter, J.

Hansmann, M.-L.

Hanson, C. A.

Hansson, U.

Haris, V.

Hartmann, S.

Hattori, C.

Hayabuchi, N.

Hayette, S.

Hebeda, K.

Heim, D

Hernandez, A. M.

Herreman, A.

Herrera M. C.

Herrera, A. G.

Heyning, F.

Hirose, Y.

Hochstenbach, F.

Hoeller, S.

Hoffman, R. M.

Hoogeboom, R.

Horie, R.

Horn, $\mathrm{H}$.

Horwitz, S.

Hosone, M.

Howayda,

Hsi, E. D.

Hsiao, C.-H.

Hsieh, Y.-C.

$\mathrm{Hu}, \mathrm{Q}$.

Huang, Y.

Huh, J.

Hummel, M.

Ibrahim, D.

Ilk, H. G.

Imre, E.-Zs.

Inghirami, G.

Iqbal, J.

Irmler, M.

Ishibashi, M.

Isimbaldi, G.

Iskas, M.

Jacob, P. M.

Jaffe, E.

Jais, J.-P

Jallades, L.

Jansen, P. M.

Jares, P.

Jarry, A.

$\begin{aligned} \text { LYS077 } & \text { Jeon, Y. K } \\ \text { LYS005, LYS073 } & \text { Jiménez, S } \\ \text { BMS002, BMS013 } & \text { Jones, D. } \\ \text { LYS001, LYS003, } & \text { Jotereau, F. } \\ \text { LYS023, } & \text { Jung, G. } \\ \text { BMS010 } & \text { Jung, I. } \\ \text { LYS010 } & \text { Jurišić, V. } \\ \text { BMS017 } & \end{aligned}$

LYS001, LYS003 K. R., A.

LYS064 Kaida, H.

LYS064 Kalpadakis, C.

BMS005 Kanellis, G.

LYS010, LYS105, LYS107 Kang, H. J.

BMS002 Kantekure, K.

LYS027 Karimi, M.

LYS033 Károlyi, K.

LYS007 Katayama, H.

LYS007 Kater, A.

LYS100 Kavantzas, N.

LYS064 Kaygusuz, G.

LYS026 Kebir, F.

LYS046 Ketterling, R. P.

BMS024 Kikuchi, T.

LYS026 Kim, H.-J.

LYS106 Kim, J. E.

LYS023, LYS082

LYS042

LYS118

ABD EL ALL LYS060

BMS023, LYS002

LYS029

LYS065

LYS017

LYS084

LYS024, LYS089, LYS091,

LYS126

LYS023

LYS060

LYS039

LYS068

BMS016, LYS003

LYS003

LYS012

LYS064

BMS004

LYS119

LYS030

LYS017

LYS006

BMS005

LYS077

LYS128

LYS016
Kim, J. M.

Kim, J. Y.

Kim, T.

Kim, Y.

Kimby, E.

Kiss, A.

Klapper, W. LYS001, LYS010, LYS023

Klier-Richter, M. LYS012

LYS001, LYS010, LYS023

Klingbiel, D.

LYS092

Kloboves-Prevodnik, V. LYS104, LYS032

Ko, Y. H.

Kobierski, J.

LYS074

Koch, K.

Koens, L.

Koh, Y.

Kolitz, J.

Konoplev, S.

Korapati, A.

Korkolopoulou, P.

Koulieris, E.

Krenacs, L.

Kuil, A.

Kumusoglu, H.

Kuo, S.-H.

Kuo, S.-Y.

Kusec, R. BMS017,

Kutok, J. L.

Kuzu, I.

Kyrtsonis, M. C.

LYS091

LYS088

LYS088

LYS009

LYS081

LYS116

LYS001

LYS077, LYS100

LYS089

BMS007

BMS018

LYS106

BMS021, LYS001,

LYS120

BMS021

LYS015

LYS083

LYS049

LYS029

LYS065

LYS035

LYS063

LYS039

BMS021
Laginestra, M. A.

Lamant, L.

LYS106

LYS016

LYS126

LYS068

LYS102

LYS030

LYS064

BMS021

LYS119

LYS89

LYS081

BMS003

LYS118

LYS026

BMS021

LYS039

BMS010

BMS012

LYS0024, LYS091

LYS0024, LYS091

\section{Lara-Torres, C.}

Laser, J.

Lassoued, K.

Lazzi, S. LYS025,

Ledoux-Pilon, A.

Lee, A.

Lee, $\mathrm{H}$.

Lee, M.

Lekakis, L.

Lemonnier, F.

León, E. A.

Leu, N.

Leung, W. Y.

Leventaki, V.

Levidou, G.

Li, C.-W.

Lia, J.-Y.

Liakou, C.

Lien, H.-C.

Lin, $\mathrm{O}$.

Lin, S.-H.

Lisfeld, J.

Liu, $\mathrm{H}$.

Lombardia, L.

López, A. B.

López, A. M.

Lord, M.

Lu, Y.-H.

Lucioni, M.

Lunning, $\mathrm{M}$.

Macho, F. M.

Macon, W.

Maestre, L.

Maffi, A.

Magaud, J.-P.

Mak, T.

Malagnino, V.

Malcovati, L.

Maldyk, J.

Maltezas, D.
LYS003, LYS013 LYS005, LYS006,

LYS010, LYS011,

LYS014

LYS061

BMS007

LYS108

LYS093

Lecaudey-Hansen, M.-H. LYS047

LeDeley, M.-C. LYS010

LYS005

LYS101

LYS091

LYS089, LYS126

LYS106

LYS006

LYS025

LYS092

LYS077

LYS106

BMS021, LYS120

LYS029

LYS029

LYS106

LYS029

LYS042

LYS065

LYS010

LYS118

LYS065

LYS061

Llamas-Gutiérrez, F.

LYS057, LYS059

LYS023

LYS114

Lome-Maldonado, C. BMS022, LYS020,

LYS057, LYS059, LYS061

Lopez-Guillermo, A. LYS007, LYS128

LYS121

LYS121

LYS081

LYS065

BMS004, LYS072

LYS042

LYS121

LYS002

LYS085

LYS072

BMS005, LYS045

LYS006

Makuch-Lasica, H. BMS019

LYS093

BMS004

LYS010

BMS021 
Mamot, C.

Manipadam, M. T.

Mannu, C.

Manso-Alonso, R.

Marafioti, T.

Maragulia, J.

Mareschal, S.

Marinos, L.

Marisavljevic, D.

Markovic, O,

Marks, R.

Marmont, F.

Martin-Garcia, N.

Martin-Moreno, A. M.

Martín-Sánche, E.

Martin, A.

Martín, C. A.

Martin, E.

Martin, L.

Martín, P.

Martínez-Benítez, B.

Martínez-Torrecuadrada, J. L

Martinez, A.

Martinez, N.

Martinovic, T.

Maryniak, R.

Matolcsy, A.

Matthews, J.

Matutes, E.

Maurer, M.

Medeiros, J.

Medinger, M.

Méhes, G.

Mehta, J.

Meignin, V.

Melendez, C.

Meloni-Ehrig, A.

Menon, $\mathrm{H}$.

Mercadal, S.

Messa, E.

Metcalf, R.

Metzler, G.

Meurette, G.

Mezei, T.

Michalak, K.

Michiels, F.

Miljić, P.

Min, S. K.

Mohammadi, F.

Molina, T. J.

Mollejo, M.

Möller, P.

Molo, S.

Momekov, G.

Monsalbez, V.
Monsalvez, V.

Montagne, K.

Montes-Moreno, S.

LYS003

LYS114

LYS001, LYS011,

LYS069

LYS042

LYS041

LYS120

LYS113

LYS102, LYS113

LYS056

BMS016

LYS046

LYS085

LYS036

LYS005, LYS006

LYS095

LYS004, LYS115

LYS005

LYS076

BMS022, LYS061

LYS085

LYS007, LYS128

LYS025, LYS115

LYS113

BMS019

LYS100

LYS101

LYS112

LYS002

BMS018, LYS070, LYS106

BMS013

BMS003

BMS014

LYS038

LYS055

LYS027

LYS034

LYS128

BMS016

LYS009

LYS044

LYS016

LYS068

BMS019

LYS005

LYS102

LYS024, LYS091

LYS103

LYS005, LYS018, LYS073

LYS037

LYS023

LYS072

BMS008

LYS115
Montes, J.

Moody, S.

Morales-Leyte, A. L.

Morales, A. L.

Morales, A. L.

Moratilla, A. B.

Moreau, A.

Morel, D.

Morettini, F.

Moro, A.

Morscio, J.

Moscinski, L.

Moskowitz, A.

Muellauer, L.

Muenst, S.

Mwanda, W.

Myskowski, P.

Nair, R.

Nair, R.

Nair, S.

Nair, S.

Naito, Z.

Nakazawa, A.

Nam, T.

Nann, S.

Narbaitz, M. I.

Naresh, K. N.

Natkunam, Y.

Navari, M.

Neme-Yunes, Y.

Nicola, M.

Nikolaou, V.

Novaković, S.

Odqvist, L.

Ogo, E.

Ogwang, M.

Ohshima, K.

Okamura, T.

Oksenhendler

Oliveira, C. C.

Ondrejka, S.

Onnis, A.

Onorati, M.

Ortiz-Hidalgo, C.

Ortiz-Romero, P. L.
Nagl, F.

Novak, K. A.
LYS127

LYS005

Ortonne, N.

Oschlies, I.

LYS047

LYS010

LYS004, LYS019, Ott, G.

LYS025, LYS037,

LYS121,LYS127

LYS019

LYS084

BMS022, LYS061

LYS057

LYS059

LYS121

LYS018

BMS005, LYS044

LYS079

BMS004

LYS033

LYS123

LYS042

LYS010

LYS092

LYS025

LYS042

LYS020

LYS030

LYS034

LYS028

LYS030

LYS118

LYS010

LYS037

BMS002

LYS095

LYS093

LYS058

LYS025

BMS022, LYS061,

LYS059

LYS072

LYS120

LYS032, LYS104

LYS032

LYS004

LYS064

LYS093

LYS064

LYS064

LYS038

LYS086, LYS096,

LYS099, LYS122, LYS125,

BMS023, LYS002

LYS025, LYS079

LYS093

LYS061

LYS127

Owen, A.

Ozbudak, I. H.

Ozcan, M.

Ozdag, $\mathrm{H}$.

Pai, R.

Paik, J. H.

Pakulniewicz, A.

Pals, S. T.

Panayiotidis, P.

Pangalis, G. A.

Papadaki, H. A.

Papadaki, T.

Papadakis, M.

Papadavid, E.

Papalexandri, A.

Papoudou-Bai, A.

Parisi, A.

Park, C.

Park, C.-S.

Park, G.

Park, S.

Parrens, M.

LYS006, LYS018,

Pascale, C.

Paschoalini, R. B.

LYS108

Passweg, J.

LYS096

Paterson, J. C. LYS069

Patey, M. LYS005

Patsouris, E. BMS021, LYS001, LYS106,

LYS 120

Pau, I.

LYS007

Paúles, M. J.

LYS055

Paulli, M,

Paunovic, I.

BMS004, LYS072

Pejsa, V.

LYS113

LYS035

Peker, D.

LYS123

Penzel, R.

BMS001

Peoch, M.

LYS005

Perry, A.

LYS017

Peruničić-Jovanović, M.

LYS102

Peterson, L. C.

BMS014

Petit, B.

Petrella, T.

LYS005

Petrovic, M. G.

Pfreundschuh, M.

Piccaluga, P. P.

Pich, A.

LYS013, LYS093, LYS079

Picquenot, J.-M.

LYSO05

Pileri, S. A.

LYS005, LYS073

LYS032, LYS104

LYS023 


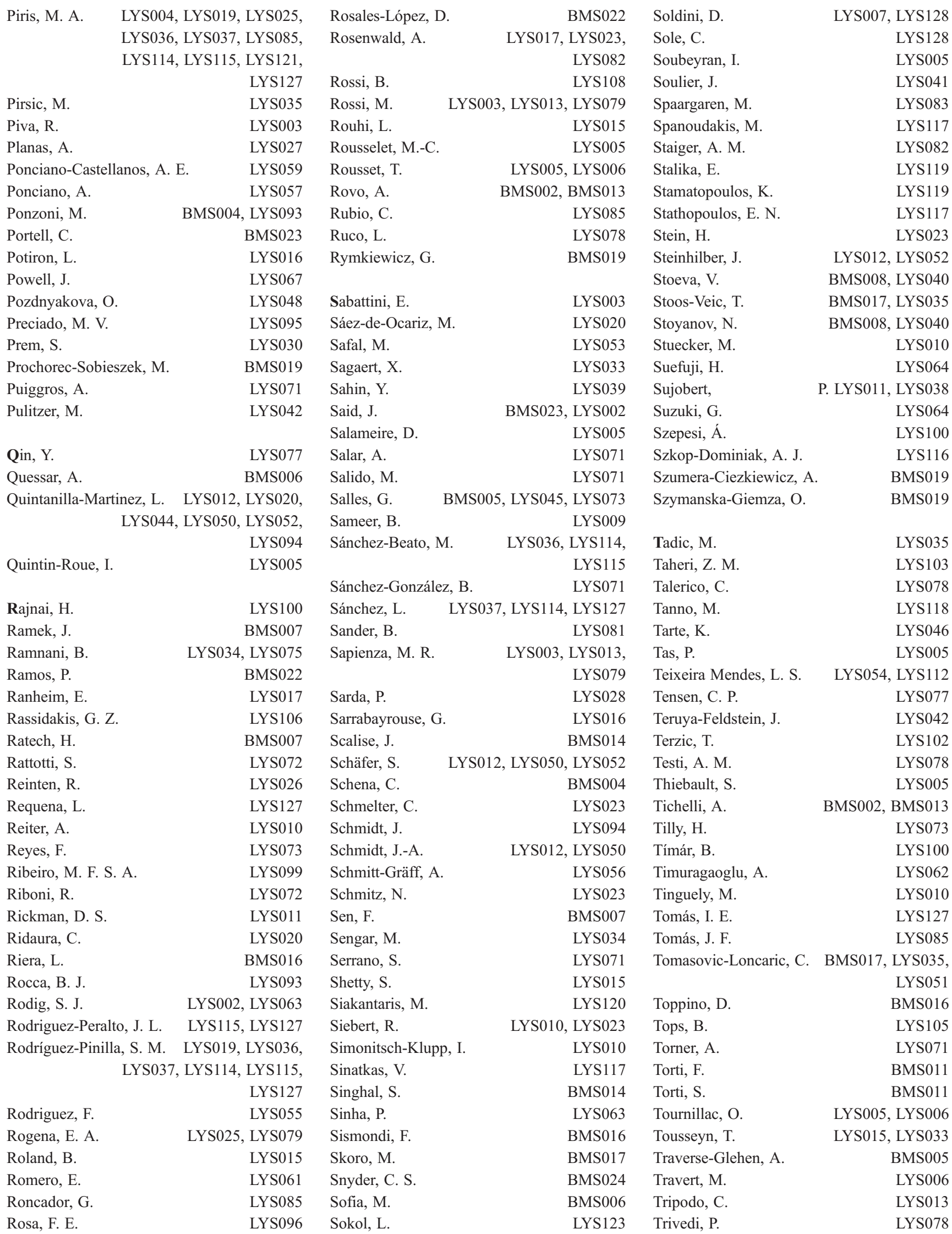


Trümper, L.

Tsakiris, D.

Tuzuner, N.

Tzankov, A.

Tzenou, T.

BMS002, BMS013, LYS070,

Uccini, S.

Üner, A.

Ungari, M.

Urbankova, H.

Valdés, D.

Valentincic, N. V.

Valera, A.

Valleron, W.

Vallina, C. M.

van der Kuip, $\mathrm{H}$.

van Kessel, K.

van Krieken, $\mathrm{H}$.

van Noesel, C.

Vandenberghe, $\mathrm{P}$.

Vaque, J. P.

Varela, I.

Varela, M.

Vassilakopoulos, T. P.

Verhoef, G.

Vermeer, M. H.

Verney, A.
LYS023

BMS002

LYS049

LYS092

BMS021

LYS078

LYS043

BMS004

LYS015

Vicente, Y.

Villaseñor-Aguirre, M.

Villaseñor, M.

Vintila, C.

Visco, C.

Viswanatha, D. S.

Vitolo, U.

Volavsek, M.

von Deimling, A.

Vrakidou, E.

Wagner, K.

BMS022, LYS059, $\quad$ Wang, H.-Y.

LYS057, LYS061 Wang, M.

LYS032, LYS104 Wang, W.

LYS007 Wang, X.

LYS014 Wang, Y.

LYS121 Warnke, R.

LYS082 Warzocha, K.

LYS026 Wasik, A. M.

LYS105, LYS107 Watanabe, Y.

LYS026 Wei, S.

LYS015 Weichert, W.

LYS115 Weisenburger, D.

LYS115 Willemze, R.

LYS055 Wilson, A.

BMS021, LYS120 Wlodarska, I.

LYS033 Wolniak, K.

LYS077 Wormhoudt, T.

BMS005 Wössmann, W.
LYS076

LYS061

BMS022, LYS059

BMS007

LYS070

BMS010

BMS016

LYS104

BMS001

LYS119

LYS026

LYS017

LYS084

BMS011

LYS081

LYS123

LYS017

BMS019

LYS081

LYS064

LYS058

BMS001

LYS017

LYS077

LYS101

LYS015, LYS033

BMS014

LYS026

LYS010
Wotherspoon, A.

Wydra, D.

LYS054, LYS112

Xerri, L.

Xirokosta, K.

Xu-Monette, Z. Y.

Xylouri, I.

Yan, Q.

Yang, $\mathrm{H}$.

Yao, M.

Yin, C. C.

Young, K. H.

Ysebaert, L.

Yu, S.-C.

Yu, Y.

LYS116

LYS005

BMS021

LYS070

LYS117

LYS084

LYS123

LYS029

BMS018

LYS070

LYS014

LYS029

LYS123

Zamiati, S.

BMS006

LYS069

LYS042

BMS020

LYS123

BMS007

LYS123

LYS058

LYS123

LYS103

LYS023

LYS081 